\title{
Abstracts of the 2017 AANS/CNS Joint Section on Disorders of the Spine and Peripheral Nerves
}

\author{
Las Vegas, Nevada • March 8-11, 2017 \\ (DOI: 10.3171/2017.3.FOC-DSPNabstracts)
}

\section{J.A.N.E Award Presentation}

100. Geriatric Co-Management Reduces Peri-Operative Complications and Shortens Duration of Hospital Stay After Lumbar Spine Surgery: A Prospective Single Institutional Experience

Owoicho Adogwa, MD, MPH; Aladine A. Elsamadicy, BE; Victoria Vuong, MS; Jessica Rose Moreno, RN, BSN; Joseph S. Cheng, MD, MS; Isaac O. Karikari, MD; Carlos Antonio Bagley, MD

Introduction: Geriatric patients undergoing lumbar spine surgery they are at risk for adverse outcomes, such as delirium, and in turn, contribute to the risk of functional decline, nursing home admission, and death. Whether pre- and peri-operative geriatric co-care reduces the incidence of in-hospital complications and length of stay after elective lumbar spine surgery remains unknown.

Methods: A unique model of shared-care for elderly patients undergoing lumbar fusion surgery was implemented at a major academic medical center. The Peri-operative Optimization of Senior Health Program (POSH) was launched with the aim of improving outcomes in elderly patients ( $>65$ years-old) undergoing lumbar spine surgery. In this model, a geriatrician evaluates elderly patients pre-operatively and follows them daily throughout the course of their hospital stay to manage medical complications and coordinate multidisciplinary rehabilitation, with neurosurgical input. We retrospectively review the first 100 cases after the initiation of the POSH-protocol and compared them with the immediately preceding 25 cases to assess the incidence of peri-operative complications and clinical outcomes.

Results: One hundred and twenty-five patients undergoing lumbar decompression and fusion were enrolled in this study. Baseline characteristics were similar between both cohorts. The length of in-hospital stay was $30 \%$ lower in the POSH cohort (6.13 vs. 8.72 days, $\mathrm{p}=0.06$ ). The duration of time between surgery and patient mobilization was significantly shorter in POSH-cohort compared to the non-POSH cohort ( 1.57 days vs. 2.77 days, $p=0.02$ ); and the number of steps ambulated on day of discharge was 2-fold higher in the POSH cohort $(\mathrm{p}=0.04)$. Compared to the non-POSH cohort, the majority of patients in the POSH cohort were discharged home (POSH:54\% vs. non-Posh:24\%, $\mathrm{p}=0.01$ ).

Conclusion: In our experience, geriatric co-management significantly reduces the incidence of post-operative complications, shortens duration of in-hospital stay, and contributes to improved peri-operative functional status in elderly patients undergoing elective spinal surgery for correction of adult degenerative scoliosis.

\section{Mayfield Basic Science Award Presentation}

101. Comparison of Pre-Operation DTI, T2SI Versus T2SI Plus DTI in a Large Series of CSM Patients for Assessment of Disease Severity and Prognostication of Recovery

Saman Shabani, BS, MD; Ha Nguyen, MD; Shekar N. Kurpad, MD, $P h D$

Introduction: Cervical spondylotic myelopathy (CSM) is a common cause of spinal cord dysfunction. Recently it has been shown diffusion tensor imaging (DTI) might be a better biomarker compare to T2 signal intensity (T2SI) on magnetic resonance imaging (MRI) for CSM. However, there has not been any study to our knowledge to assess combination of both T2SI and DTI in conjunction to determine disease severity and recovery.

Methods: A retrospective analysis of 44 patients with preoperative DTI was done. Presence or absence of T2SI at the level of maximum compression (LMC) was determined. Normalized T2SI (NT2SI) regardless of presence or absence of T2SI at (LMC) was determined by calculating T2SI at LMC/T2SI at level of foramen magnum. LMC NT2SI, +/- T2SI, and fractional anisotropy (FA) were obtained and correlated to preop mJOA and ?mJOA at 3, 6, 12, 24 months. Regression analysis and independent t-tests were used for analysis of the data.

Results: There was a significant correlation between preop mJOA and LMC FA $(\mathrm{P}=0.048)$. There was no significant correlations between preop $\mathrm{mJOA}$ and presence of T2SI. With regards to delta mJOA, significant relationships were discovered with LMC FA at 12 months $(p<0.05)$; on the contrary, there were no significant relationships associated with NT2SI or presence of T2SI. Combining NT2SI or presence of T2SI to LMC FA in multivariate linear regression analysis also did not improve the predictive value significantly, compared to using LMC FA alone.

Conclusion: In this larger retrospective study of CSM patients, FA at LMC shows to be a better biomarker for determining the disease severity, and both short and long term outcomes compared to T2SI at LMC. Moreover, combined FA and T2SI did not have superiority compare to FA alone in these subgroup of patients.

\section{Mayfield Clinical Science Award Presentation}

102. Intrathecal Morphine Following Lumbar Fusion: A Randomized, Placebo-Controlled Trial

Daniel Yavin, MD; Perry Pawandeep Singh Dhaliwal, MD; Tara Whittaker, BN; Geoffrey S. Hawboldt, MD; Gordon Jewett, BSc, PhD; Steven Casha, MD, PhD; Stephan Jean du Plessis

Introduction: Despite the potential for faster postoperative recovery and the ease of direct intraoperative injection into the exposed dura, intrathecal morphine is rarely provided in lumbar spine surgery.

Methods: In this double-blind trial, we randomly assigned 150 patients undergoing instrumented lumbar fusion for degenerative indications to receive a single intrathecal injection of morphine $(0.2 \mathrm{mg})$ or placebo (normal saline) immediately prior to wound closure. An oblique injection technique was used to reduce the risk of precipitating a cerebrospinal fluid leak. The primary outcome was pain measured on the visual-analogue scale during the first 24 hours after surgery. Secondary outcomes included respiratory depression and treatment-related side effects. An intention-to-treat, repeatedmeasures analysis was used to estimate outcomes.

Results: The baseline characteristics of the groups were similar. Intrathecal morphine reduced pain both at rest $(32 \%$ area under the curves $[\mathrm{AUCs}$ ] difference, $\mathrm{P}<0.002)$ and with movement $(22 \%$ AUCs difference, $\mathrm{P}<0.02$ ) during the initial 24 hours after surgery. The risk of respiratory depression was not increased by intrathecal morphine 
(hazard ratio, $0.86 ; 95 \%$ confidence interval, 0.44 to $1.68 ; \mathrm{P}=0.66$ ). Postoperative opioid requirements were reduced with intrathecal morphine $(\mathrm{P}<0.03)$. Other than a trend towards increased intermittent catheterization in patients assigned to intrathecal morphine $(\mathrm{P}=0.09)$, treatment-related side effects did not significantly differ between the groups. The early benefits of intrathecal morphine on postoperative pain were no longer apparent after 48 hours.

Conclusion: A single intrathecal injection of $0.2 \mathrm{mg}$ of morphine safely reduces postoperative pain following lumbar fusion. (Funded by the Alberta Spine Foundation; ClinicalTrials.gov number, NCT01053039.)

\section{Kline Peripheral Nerve Award Presentation}

\section{Prediction Algorithm for Surgical Intervention in Neonatal Brachial Plexus Palsy}

\section{Thomas J. Wilson, MD; Kate Chang; Lynda Jun-San Yang, MD, PhD}

Introduction: Neonatal brachial plexus palsy (NBPP) results in reduced function of the affected arm with profound ramifications on quality of life. Advances in surgical technique have shown improvements in outcomes for appropriately selected patients. Patient selection, however, remains difficult. Our objective was to develop a decision algorithm that could be applied at the individual patient level, early in life, to reliably predict persistent NBPP that would benefit from surgery.

Methods: Retrospective review of NBPP patients was undertaken. Maternal and neonatal factors were entered into the C5.0 statistical package in R. A 60/40 model was employed, whereby $60 \%$ of randomized data were used to train the decision tree, while the remaining $40 \%$ were used to test the decision tree. The outcome of interest for the decision tree was a severe lesion meeting requirements for surgical candidacy.

Results: A decision tree prediction algorithm was generated from the entered variables (Figure 1). Variables utilized in the final decision tree included presence of Horner's syndrome, presence of a pseudomeningocele, Narakas grade, clavicle fracture at birth, birth weight $>9 \mathrm{lbs}$., and induction or augmentation of labor. Sensitivity of the decision tree was 0.71 , specificity 0.96 , positive predictive value 0.94 , negative predictive value 0.79 , and F1 score 0.81 .

Conclusion: We developed a decision tree prediction algorithm that can be applied shortly after birth to determine surgical candidacy of patients with NBPP, the first of its kind utilizing only maternal and neonatal factors. This conservative decision tree can be used to offer early surgical intervention for appropriate candidates.

\section{Charles Kuntz Scholar Award Presentations (Abstracts 104-123)}

104. Minimally Effective Dose of Bone Morphogenetic Protein (BMP) in Minimally Invasive (MIS) Lumbar Interbody Fusions 757 Patients in a Dose-finding Statistical Modeling Cohort Study

Evan Joseph Lytle, DO; Doris Tong, MD; Lisa Govila; Roger F. Gonda, MD; John Nofar, BA; Clifford Michael Houseman, DO; Teck-Mun Soo, MD

Introduction: With increasing doses of BMP, the risk of adverse events increases. We seek to demonstrate the minimal effective BMP dose to achieve a fusion in MIS.

Methods: From 2009-2014, we reviewed the charts of consecutive patients who underwent MIS lumbar Interbody fusion in a longitudinal cohort study. We excluded patients without radiographic (XR) follow up $=3 \mathrm{~m}$ postop. Dose of BMP/interspace was determined. Fusion was determined by XR evaluated by neuroradiologists. A pilot study was performed to determine the baseline fusion rate in our local population for a sample size with $\mathrm{a}=0.05$ and power $=0.8$. We used multiple logistic regression with fusion $=3 \mathrm{~m}$ and $=6 \mathrm{~m}$ as the dependent variable and BMP dose per interspace, single vs. multilevel, specific level, postop XR interval, smoking, gender, age as covariates. We satisfied the assumption tests and Wald test was used. The $\operatorname{Exp}(\beta)$ coefficient was used to show change in the odds of fusion for one-unit change in an independent variable when all other independent variables are kept constant. Minimally effective dose of $\mathrm{BMP} /$ level was determined by $95 \%$ CI between different dose ranges. We considered a p-value of 0.0125 to compensate for multiple comparisons.

Results: There were 858 interspaces among 757 unique patient encounters (Table 1). Average dose was $1.08 \mathrm{mg} /$ level (Table 2). Gender, dose/level, and single vs. multi-level fusion were significant predictors for fusion $=3 \mathrm{~m}$ postop (Table 3 ). Dose $/$ level and Single vs. multi-level fusion were significant predictors for fusion $=6 \mathrm{~m}$ postop (Table 4). Overall fusion rate $=3 \mathrm{~m}$ postop was $93.8 \%$ (95\% CI $0.92-$ 0.95 ) (Table 5) vs. $93.5 \%$ (95\% CI 0.92-0.95) (Table 6$)$ at $=6 \mathrm{~m}$ postop.

Conclusion: BMP dose/level and single vs. multi-level fusion were significant predictors for fusion $=6 \mathrm{~m}$ postoperatively. We found that the effective fusion rate of $91 \%$ (95\%CI: 0.87-0.95) was achieved at dose less than $1.05 \mathrm{mg}$ (size of XXS) per level.

105. Vitamin D in Multi-level Anterior Cervical Fusion: Interim Results from a Multi-Center Comparative Effectiveness Study

Doniel Drazin, MD, MA; Christine Piper; Zachary Rollins Barnard, MD; Lorenzo Rinaldo, MD, PhD; Alexandre Rasouli, MD; Mohamad Bydon, MD; William E. Krauss, MD; Michelle J. Clarke, MD; Joseph R. O'Brien, MD, MPH; Warren Yu; Edward K. Nomoto, MD; Daniel Norvell; Ray M. Chu, MD; Robert S. Pashman, MD; Eli M. Baron, MD; Terrence T. Kim, MD; J. Patrick Johnson, MD, MS

Introduction: Vitamin D deficiency is common in patients presenting for spinal surgery. It has been unclear whether this abnormality affects spinal fusion outcomes.

Methods: In a multi-center prospective comparative effectiveness study, we assessed vitamin D levels in patients undergoing multilevel anterior cervical discectomy and fusion. Exclusion criteria included previous cervical spine surgery, posterior approach and bone morphogenic protein product use. The primary outcome measure was vitamin D level. Participants were classified into two groups based on CDC criteria: normal $(>30 \mathrm{ng} / \mathrm{mL})$ or subnormal $(<29.9 \mathrm{ng} / \mathrm{mL}$ ) level, determined by vitamin D (serum $25-\mathrm{OHD})$ test. Post-operative fusion was assessed by upright lateral cervical spine flexion-extension radiographs. Patients were followed for 1 year.

Results: Of 97 enrolled patients, 42 have had complete followup. There were no significant differences in baseline or operative characteristics between the groups. A greater proportion of patients with vitamin D deficiency underwent revision (86\%) compared to those with normal levels (17\%). This was statistically significant $(p=.007)$. Mean vitamin D level of revision patients was $25.3 \pm$ 9.0 compared to $35.7 \pm 15.5$ for non-revision patients. This was statistically significant $(p=.04)$. There was no statistically significant association between age, BMI, gender, length of stay, or surgical level and the risk for revision. In patients with spondylolysis as their primary diagnosis, the revision risk was $50 \%(n=6)$ compared to $13 \%$ $(n=4)$ for non-spondylolysis patients. All 12 revisions occurred in non-smokers. In a multivariable logistic regression model, patients with normal vitamin D levels were at lower odds of undergoing revision. This was statistically significant (odd ratio $=.04,95 \%$ confidence interval: .004-.39; $\mathrm{p}=.006$ ). 
Conclusion: Our interim analysis indicates that vitamin D levels do correlate with spinal fusion outcomes. To maximize chances for successful arthrodesis and optimal surgical outcomes, we recommend that vitamin D insufficiency be addressed and corrected.

\section{The Inescapability of Variation in Outcomes After Lumbar Spine Surgery: Insights from the National Neurosurgery Quality and Outcomes Database}

Ahilan Sivaganesan, MD; Silky Chotai, MD; Matthew J. McGirt, MD; Anthony L. Asher, MD, FACS; Clinton J. Devin, MD

Introduction: Low-back surgery has come under scrutiny due to concerns over efficacy and over-use. Never before has an attempt been made to quantify and visualize the variability in lumbar surgery outcomes on a national scale. Here, we present such an effort.

Methods: Data was collected from 28 N2QOD institutions which had completed 12-month patient follow-up (yielding 10,864 patients). Descriptive statistics were computed for an array of baseline characteristics and patient-reported outcomes (PRO). Variation in "change scores" for PROs were computed. The Student's t-test was used to determine whether there were significant improvements in PROs from baseline to 12 months after surgery.

Results: There was a significant improvement in ODI from baseline to 12 months after surgery when all patients were pooled together $(p<0.001)$. This was also the case for EQ5D, VAS back pain, and leg pain. These improvements were also seen for every PRO when patients were stratified by diagnosis. However, marked variation in outcomes did exist - for example, $29 \%$ of patients did not achieve a MCID in ODI. Similar findings were seen for EQ5D, back pain, and leg pain. This variability in achieving MCID was seen at each of the 28 N2QOD sites. Scatterplots were constructed to visualize this variability for each PRO.

Conclusion: This study is the first attempt, at a national scale, to characterize variability in PROs after elective spine surgery. Such a study was not possible in the past, because a data infrastructure with the reach and granularity of N2QOD did not previously exist. We have established that elective lumbar surgery is invariably ineffective for a non-trivial proportion of individuals, and that this phenomenon persists regardless of diagnosis, the outcome examined, or geographic location. Analytics tools built on the understanding of PRO variability established here will help neurosurgeons offer more predictable care.

107. Radiological Predictors of Kyphosis After Thoracolumbar Burst Fractures Managed Using Thoracolumbarsacral Orthosis and Long-term Follow-up of Outcomes

Michael M.H. Yang, MD, M.Biotech; Baldeep Dulko, BSc; Jacob Oh; Steven Casha, MD, PhD

Introduction: Thoracolumbar burst fractures (TLBF) in neurologically intact patients can be treated non-operatively with an orthosis. This study aims to determine the radiological predictors of progressive kyphosis after TLBF in patients managed with a thoracolumbarsacral orthosis and to correlate clinical outcomes with severity and progression of kyphotic deformity.

Methods: We retrospectively identified patients who during 2008-2012 sustained a thoracolumbar burst fractures and managed non-operatively using an orthosis. Sagittal cobb-angle, coronal cobb-angle, sagittal canal diameter, facet apposition, interpedicular distance, anterior/mid/posterior-body height loss and maximum retropulsed fragment were determined. Measurements were made on initial CT-scan (CT), initial upright X-ray (XR1) and follow-up upright X-ray at $>3$ months (XR2). Long-term clinical outcomes were collected (SF-36, Oswestry Disability Index (ODI) and visual analogue scale (VAS)).

Results: 95 patients were included in the study. The mean sagittal cobb-angles on CT, XR1, and XR2 were $10.3,11.3,14.9^{\circ}$ respectively. The mean change in cobb-angle between each time point was: CT-XR1, $1.0^{\circ}(\mathrm{NS})$; XR1-XR2, $3.6^{\circ}(\mathrm{p}<0.001)$; CT-XR2, $4.6^{\circ}(\mathrm{p}<0.001)$. The average time between CT and XR2 was 9.2 months. There was significant correlation between initial CT anterior/mid/posterior-body height loss and interpedicular distance to progression of kyphosis $(\mathrm{p}=0.047)$. There was no correlation between initial sagittal cobb-angle, magnitude of retropulsed fragment, facet apposition, coronal cobb-angle and sagittal canal diameter to progression of kyphosis. 71 patients $(75 \%)$ completed the clinical outcome surveys. In that subset of patients, no significant correlation was seen between SF-36, ODI, VAS and initial, final or progression of kyphosis. Mean time from injury to health outcomes questionnaires was 64 months.

Conclusion: The majority of TLBFs treated with a thoracolumbarsacral orthosis exhibit little progressive kyphotic deformity. Anterior/mid/posterior body height loss and increased interpedicular distance were predictors of sagittal kyphotic progression. The magnitude of kyphosis does not correlate with clinical outcomes.

108. Outcomes of Surgery For Sacral Chordoma and Impact of Complications: A Report of 50 Consecutive Patients with Longterm Follow-up

Scott L. Zuckerman, MD; George Chang, MD; Garrett L. Walsh; Reza Mehran; Richard George Everson, MD; Sung-Ho Lee, MD; Ganesh Rao, MD; Claudio E. Tatsui, MD; Laurence D. Rhines, MD

Introduction: Surgical resection of sacral chordomas improves local control; however, the morbidity of an en bloc resection is considerable. The current study aims to determine predictive factors of overall survival (OS) and local recurrence (LR), report perioperative complications, and assess the impact of complications on OS and LR.

Methods: This retrospective case series was obtained from a prospectively maintained spine database from 1995-2016. All patients who underwent en bloc resection of sacral chordoma were included. Detailed demographic, perioperative, and complication data was collected. In addition to descriptive statistics, a survival analysis was completed with multivariable cox regression to assess the impact of predictive factors and complications on OS and LR.

Results: A total of 50 patients were included. Median follow-up was 63 (15-206) months. The majority of patients (82\%) underwent en bloc resection with negative margins. Survival: a total of 17 patients died $(34 \%)$ with a median OS of 10 years. Multivariable cox regression revealed that en bloc resection with negative margins predicted longer survival $(\mathrm{HR}=3.35, \mathrm{p}=0.078,95 \%$ CI $0.87,12.80)$; however, this was only marginally significant. Recurrence: a total of 20 patients $(40 \%)$ experienced LR with a median time of 6.2 years. Multivariable cox regression revealed that en bloc resection with negative margins predicted a decreased likelihood of LR $(\mathrm{HR}=4.96, \mathrm{p}=0.002,95 \% \mathrm{CI} 1.84,13.34)$. We reported a $62 \%$ overall complication rate. Thirteen patients $(26 \%)$ required reoperation. After multivariable cox regression, neither major complication nor reoperation significantly impacted OS or LR.

Conclusion: In the current single-institution series of en bloc resection for sacral chordoma, a negative margin resection significantly improved LR and was marginally associated with OS. Neither major complication nor reoperation significantly impacted OS or LR. Thus, it appears the inherent high surgical morbidity associated with these invasive operations does not significantly alter the trajectory of survival and recurrence. 
109. Quantitative Multi-Parametric Spinal Cord MRI Detects Subclinical Tissue Injury in Asymptomatic Cervical Spinal Cord Compression

Allan R. Martin, BASc, MD; Benjamin De Leener; Julien CohenAdad; David W. Cadotte, MSc, MD; Jefferson R. Wilson, MD, PhD; Lindsay Tetreault, Bsc; Aria Nouri, MD, MSc; Adrian Crawley, PhD; David J. Mikulis, MD; Howard J. Ginsberg, BASc, MD, PhD, FRCSC; Michael G. Fehlings, MD, PhD, FRCS(C), FACS

Introduction: Degenerative cervical myelopathy (DCM) is a condition in which extrinsic compression and dynamic injury cause spinal cord (SC) tissue injury and neurological dysfunction. Degenerative SC compression has also been observed in $8-26 \%$ of the asymptomatic population [1,2], increasingly with age. Quantitative MRI accurately quantifies SC tissue injury in DCM [3], but it is unknown if asymptomatic patients with cord compression also experience similar changes.

Methods: 32 subjects without neurological symptoms/signs underwent T2-weighted (T2w), diffusion tensor imaging (DTI), magnetization transfer (MT), and T2*-weighted (T2*w) MRI (3T) covering C1-C7. Subjects were grouped based on presence of cord compression (indentation, flattening, or focal torsion). SC crosssectional area (CSA), fractional anisotropy (FA), MT ratio (MTR), and $\mathrm{T} 2 * \mathrm{w}$ white to grey matter signal intensity ratio $\left(\mathrm{T} 2 *_{\mathrm{W}} \mathrm{WM} /\right.$ GM) were calculated at maximally compressed (MCL), rostral (C1-C3), and caudal (C6-7) levels and normalized for confounding variables (including age). One-tailed $\mathrm{T}$ tests and a single binomial test assessed if asymptomatic compression caused similar changes in 10 metrics as previously observed in DCM $(\mathrm{N}=56)$. Area under receiver operating characteristic curves (AUC) measured diagnostic accuracy.

Results: SC compression subjects $(\mathrm{N}=12,38 \%)$ were older than uncompressed subjects (53.9 vs. 38.9, $\mathrm{p}=0.004)$ and showed decreased rostral MTR (50.8 vs. 53.5, $\mathrm{p}=0.005, \mathrm{AUC}=0.765)$, decreased MCL MTR (T statistics: -0.255 vs. $0.254, p=0.05$, AUC $=0.604$ ), and increased rostral $\mathrm{T} 2{ }^{*} \mathrm{~W} \mathrm{WM} / \mathrm{GM}(0.875$ vs. $0.857, \mathrm{p}=0.05$, $\mathrm{AUC}=0.675$ ). The other 7 metrics did not show significant differences. The directional pattern of changes matched differences seen in DCM in $8 / 10$ measures $(p=0.05)$.

Conclusion: Asymptomatic individuals with spinal cord compression show macro- and microstructural changes similar to DCM subjects, suggesting the presence of subclinical tissue injury. While these results require further validation, they offer the intriguing possibility of injury detection prior to the onset of clinical symptoms and signs. These findings have far-reaching implications, including earlier diagnosis and treatment for all spinal pathologies.

110. Congenital Cervical Spine Stenosis in a Global Cohort of Patients with Degenerative Cervical Myelopathy: A Report Based on a MRI Diagnostic Criterion

Aria Nouri, MD, MSc; Lindsay Tetreault, Bsc; Allan R. Martin, BASc, MD; Anick Nater, MD, BS; Satoshi Nori, MD; Michael G. Fehlings, $M D, P h D, F R C S(C), F A C S$

Introduction: Congenital Spinal Stenosis (CSS) is a known predisposing factor for Degenerative Cervical Myelopathy (DCM). However, current diagnostic criteria for CSS do not consider the size of the spinal cord, and methods to establish pre-existing CSS in patients with DCM do not presently exist. Using a global cohort of patients with DCM, MRI-based criteria were developed to diagnose pre-existing CSS and to evaluate differences between patients with and without CSS.

Methods: Study data (including 349 MRIs for quantitative analysis) were derived from two international prospective and multicenter studies collected between 2005-2011. Spinal canal and cord anteroposterior diameters were measured above and below the region of interest at non-compressed sites, and a spinal cord occupation ratio (SCOR) was calculated. A SCOR $=70 \%$ was used to diagnose patients with CSS. Torg-Pavlov ratios and spinal canal diameters from radiographs were correlated with SCOR. Clinical and MRI factors were compared between patients with and those without CSS using t-tests. Multiple linear regression was used to assess surgical outcome.

Results: Calculation of SCOR was feasible in 311/349 patients (89\%). Twenty-six patients with CSS were identified (8.4\%). Patients with CSS were younger than patients without CSS (50.8 vs. 56.3, $\mathrm{p}=0.03$ ) and had worse baseline severity as measured by the mJOA $(p=0.04)$, Nurick $(p=0.05)$ and NDI $(p<0.01)$. CSS patients also presented more commonly with $\mathrm{T} 2$ cord hyperintensity changes $(p=0.09)$, and worse SF-36 Physical Component scores $(p=0.06)$, though this did not reach statistical significance. SCOR was correlated with Torg-Pavlov ratio and spinal canal diameter at $\mathrm{C} 3$ but not $\mathrm{C} 5$. Patients with a $\mathrm{SCOR}=65 \%$ were also younger but did not have differences in baseline severity.

Conclusion: CSS patients develop myelopathy at a younger age and have greater impairment and disability than other patients with DCM. Despite this, CSS patients have comparable duration of symptoms, MRI presentations, and surgical outcomes to DCM patients without CSS.

111. Preoperative Skin Antisepsis with Chlorhexidine Gluconate Versus Povidone-Iodine: A Prospective Analysis of 6959 Consecutive Spinal Surgery Patients

George M. Ghobrial, MD; Michael Y. Wang, MD, FACS; Barth A. Green, MD, FACS; Howard B. Levene, MD, PhD; Glen R. Manzano, MD; Steven Vanni, DO; Robert M. Starke, MD, MSc; George Jimsheleishvili, MD; Kenneth M. Crandall, MD; Marina Dididze, $M D, P h D ;$ Allan D. Levi, $M D, P h D$

Introduction: The efficacy of two common preoperative surgical skin antiseptic agents, ChloraPrep ${ }^{\circledR}$ and Betadine ${ }^{\circledR}$ have not been previously evaluated regarding the reduction of postoperative surgical site infection in spinal surgery procedures.

Methods: Two common preoperative surgical skin-antiseptic agents were prospectively compared for all consecutive adult neurosurgical spine patients: ChloraPrep ${ }^{\circledR}(2 \%$ chlorhexidine gluconate and 70\% isopropyl alcohol; CareFusion, Inc., Leawood, KS), and Betadine ${ }^{\circledR}$ (7.5\% povidone-iodine solution, Purdue Products L.P., Stamford, CT), across two consecutive time periods. The primary endpoint was the incidence of surgical site infection (SSI).

Results: A total of 6959 consecutive spinal surgery patients were identified from July 1st, 2011 through August 31st, 2015 with $4495(64.6 \%)$ and 2464(35.4\%) patients treated at facilities 1 and 2, respectively. There were $69(0.992 \%)$ surgical site infections observed. There was no significant difference in the incidence of infection in those patients prepped with betadine (33 of 3,185; 1.036\%) versus those that received ChloraPrep ${ }^{\circledR}(36$ of 3,$774 ; 0.953 \% ; p=0.728)$. There was no significant difference in the incidence of infection in patients treated at facility 1 ( 52 of 4,$495 ; 1.157 \%)$ versus patients treated at facility 2 (17 of 2,$464 ; 0.690 \%)$. Of the SSI patients, the most common indication was degenerative disease (45 of 69, 65\%). Fifty-one $(74 \%)$ patients with SSI were associated with instrumented fusions in the index operation and 38(55\%) patients with SSI were revision surgeries. The incidence of SSI for minimally-invasive and open surgery was $0.226 \%(\mathrm{n}=2)$ and $1.103 \%(\mathrm{n}=67)$, respectively.

Conclusion: The choice of either ChloraPrep $\AA$ or Betadine $(\AA$ for preoperative skin antisepsis in spinal surgery had no significant impact on the incidence of postoperative surgical site infection. 
112. Development of a Preoperative Metastatic Spinal Tumor Frailty Index (MSTFI) Using a Nationwide Database and Its Association with Inpatient Morbidity, Mortality, and Length of Stay

C. Rory Goodwin, MD, PhD; Rafael De la Garza-Ramos, BA; Amit Jain, BS; Taylor Elise Purvis, BA; Nancy A. Abu-Bonsrah, BS; Charles G. Fischer; Benjamin D. Elder, MD, PhD; A. Karim Ahmed; Chetan Bettegowda, MD, PhD; Daniel M. Sciubba, MD

Introduction: The aim of this study was to develop a perioperative metastatic spinal tumor frailty index (MSTFI) that could predict morbidity, mortality, and length of stay.

Methods: A large inpatient hospitalization database was searched from 2002 to 2011 to identify 4583 patients with spinal metastasis from breast $(21.1 \%)$, lung $(34.1 \%)$, thyroid $(3.8 \%)$, renal $(19.9 \%)$, and prostate $(21.1 \%)$ cancer who underwent surgery. A multiple logistic regression model identified 9 independent parameters that were used to construct the MSTFI: anemia, chronic lung disease, coagulopathy, electrolyte abnormalities, pulmonary circulation disorders, renal failure, malnutrition, emergent/urgent admission, and anterior/ combined surgical approach. Patients with 0 points were categorized as not frail, 1 as mildly frail, 2 as moderately frail, and $=3$ as severely frail.

Results: The overall perioperative complication rate was 19.3\% and in-patient mortality was $3.0 \%$. Compared with patients with no frailty, patients with moderate frailty (odds ratio [OR] 5.15; 95\% confidence interval [95\% CI] 2.44-10.86), and severe frailty (OR 5.74; 95\% CI 2.69-12.24) had significantly increased odds of inpatient mortality (all $\mathrm{P}<0.001)$. Similarly, patients with mild frailty (OR 1.88; 95\% CI 1.33-2.66), moderate frailty (OR 3.83; 95\% CI 2.71-5.41), and severe frailty (OR 6.97; 95\% CI 4.98-9.74) had significantly increased odds of developing a major in-hospital complication (all $\mathrm{P}<0.001$ ). Length of stay also increased significantly by MSTFI $(\mathrm{P}<0.001)$.

Conclusion: In surgically treated patients with spinal metastasis, certain perioperative parameters may significantly predict the risk of major in-hospital complications and mortality.

\section{Quality of Life After Spinal Cord Injury - Results from a Canadian National Cross-sectional Survey}

Christian Iorio-Morin, MD, PhD; Vanessa Noonan; Barry White; Luc Noreau; Jean Leblond; Frédéric S. Dumont; Nicolas Dea, MD, FRCSC

Introduction: Traumatic spinal cord injury (SCI) is a leading cause of worldwide disability. Underlying all medical and physical interventions is the assumption that maximizing neurological function and improving functional autonomy will improve the patient's longterm quality of life (QoL). The goal of this study was to provide overall QoL, health-related QoL and health utility values for patients with traumatic SCI stratified by injury level and neurological status.

Methods: The Canadian SCI Community Survey was sent to Canadians living in the community following SCI. The survey covered demographics, SCI classification, secondary complications, comorbidities, needs, community participation, activities and employment, QoL, health care utilization, overall health status and overall QoL. The impact of demographics, complications and SCI classification on QoL was assessed using ANOVA, multiple linear regressions and ordinal logistic regression analyses.

Results: There were 1109 respondents with traumatic SCI. 70\% were male. Mean age was 48.3 years at a mean of 18.5 years following injury. ASIA Impairment Scale grade was cervical A or B in $20 \%$, cervical $\mathrm{C}$ or $\mathrm{D}$ in $28 \%$, thoracolumbar $\mathrm{A}$ or $\mathrm{B}$ in $32 \%$, thoracolumbar $\mathrm{C}$ or $\mathrm{D}$ in $16 \%$ and $\mathrm{E}$ (any level) in $1 \%$. Injury level or AIS grade had no impact on either health utility or QoL. Factors affecting QoL included a depressed mood, fatigue, injuries caused by a loss of sensation, not working, diabetes, concomitant renal disease, neuropathic pain, constipation, sleep problems, hypertension, sexual dysfunction, pressure ulcers and joint contractures. With a mean health utility score of $0.64 \pm 0.12$, SCI patients living in the community reported having QoL similar to patients with rhinosinusitis, symptomatic hand osteoarthrosis and healed hip fracture.

Conclusion: Injury level and AIS grade do not affect long-term QoL. Therefore, QoL should not be used as an outcome to assess the effectiveness of interventions targeting neurological function and autonomy in traumatic SCI.

114. Optical Topographic Imaging for Intra-Operative ThreeDimensional Navigation in the Cervical Spine: Accuracy Validation and Initial Clinical Feasibility

Daipayan Guha, MD; Raphael Jakubovic, MSc; Shaurya Gupta; Michael G. Fehlings, MD, PhD, FRCS(C), FACS; Albert Yee, MD; Victor Xiao Dong Yang, $M D, P h D, M S c, B S c$

Introduction: Computer-assisted three-dimensional navigation may guide spinal instrumentation. Current systems are hampered by cumbersome registration and inability to account for intra-operative tissue movement. A novel optical topographic imaging (OTI) system was developed for craniospinal neuronavigation, and has been described previously in the thoracolumbar spine. Here, we validate its accuracy in the mobile cervical spine.

Methods: Initial validation was performed in four human cadavers. Intra-operative registration was performed to thin-slice preoperative CT imaging. A tracked drill guide was used to navigate screw tracts at all levels. Lateral mass screws were placed at $\mathrm{Cl}$ and C3-6, pars screws at $\mathrm{C} 2$, and pedicle screws at C7. Navigation data were compared to screw positions on postoperative CT imaging, and the absolute translational and angular deviations computed (Fig. 1). Clinical validation was subsequently performed in six patients undergoing open posterior cervical instrumentation.

Results: 53 cadaveric screws were analyzed; 5 lateral mass screws at $\mathrm{C} 1$ and 32 at $\mathrm{C} 3-6,8$ pars screws at $\mathrm{C} 2$, and 8 pedicle screws at $\mathrm{C} 7$. Absolute translational errors were $1.66+/-1.18 \mathrm{~mm}$ and $2.08+/-2.21 \mathrm{~mm}$ in the axial and sagittal planes, respectively; absolute angular deviations were 4.11+/-3.79deg and 6.96+/-5.40deg, respectively (mean+/-SD). In hierarchical linear modelling, adjusting for differences between cadavers, C7 pedicle screws demonstrated decreased axial translational error relative to all other screws $(0.51+/-$ $0.36 \mathrm{~mm}, \mathrm{p}=0.001)($ mean $+/ \mathrm{SD}) .22$ clinical screws were analyzed; 2 pars screws at $\mathrm{C} 2,14$ lateral mass screws at $\mathrm{C} 3-5$, and 6 pedicle screws at C7. Absolute translational errors were $1.52+/-1.32 \mathrm{~mm}$ and $1.06+/-0.97 \mathrm{~mm}$ in the axial and sagittal planes, respectively; absolute angular deviations were $3.69+/-2.63 \mathrm{deg}$ and $2.83+/-2.65 \mathrm{deg}$, respectively (mean+/-SD). There were no differences in errors between levels. There were no facet, canal or foraminal violations, and no neurovascular injuries.

Conclusion: Optical machine-vision is a novel navigation technique allowing efficient initial and repeat registration. Accuracy even in the more-mobile cervical spine is comparable to current spinal neuronavigation systems. 
115. Patient-Reported Outcomes After Surgical Stabilization of Spinal Tumors: Symptom-Based Validation of the Spinal Instability Neoplastic Score (SINS) and Surgery

Ibrahim Hussain; Ori Barzilai, MD; Anne Reiner; Natalie DiStefano, BA; Lily McLaughlin; Shahiba Ogilvie, MPH; Mark H. Bilsky, MD; Ilya Laufer, MD

Introduction: Neoplastic spinal instability is defined as movementrelated pain, deformity, or neurologic compromise under physiologic loads with SINS developed to facilitate diagnosis. There is a paucity of evidence that mechanical instability correlates with pain and disability and that surgical stabilization significantly improves these patient-reported outcomes (PRO).

Methods: SINS and PRO (Brief Pain Inventory (BPI) and MD Anderson Symptom Inventory Spine Tumor (MDASI-SP)) from patients who underwent instrumented surgical stabilization for spinal tumor treatment were prospectively collected. SINS was analyzed as a continuous and ordinal categorical variable (Stable 0-6, Low Indeterminate 7-9, High Indeterminate 10-12, Unstable 13-18). Association between SINS and pre-operative symptoms was analyzed using Spearman Rank Coefficient (rho) and an extension of the Cochran-Armitage trend test, with $\mathrm{p}$-values $<0.05$ considered significant.

Results: 150 surgeries were performed with the average postoperative assessment at 35 days. There was a statistically significant positive correlation between increasing pre-operative SINS and the severity of pre-operative pain as measured by BPI worst pain $(\mathrm{rho}=0.18, \mathrm{p}=0.02)$, BPI average pain $(\mathrm{rho}=0.23, \mathrm{p}=0.005)$, and MDASI pain (rho $=0.2, \mathrm{p}=0.01$ ) items. Increasing pre-operative SINS also correlated with increasing severity of pre-operative disability measured by BPI walking (rho $=0.23, \mathrm{p}=0.006$ ), MDASI activity $(\mathrm{rho}=0.28, \mathrm{p}=0.0006)$, and MDASI walking $(\mathrm{rho}=0.22, \mathrm{p}=0.009$ ) items. Similar associations were noted when SINS was analyzed as an ordinal categorical variable. The magnitude of symptom relief after surgery positively correlated with pre-operative SINS, as patients with higher pre-operative SINS experiencing greater symptom relief with BPI worst pain ( $\mathrm{p}=0.03)$, BPI average pain $(\mathrm{p}=0.02)$, BPI activity $(\mathrm{p}=0.04)$, and MDASI pain $(\mathrm{p}=0.04)$ (Figure 1$)$.

Conclusion: The association between increasing SINS and symptom burden provide PRO-based validation of SINS as a diagnostic instrument for spinal instability. The larger decrease in the severity of pain and activity interference in patients with neoplastic spinal instability supports the role of surgical stabilization in the setting of mechanically unstable spines.

116. The Effect of Steroids on Complications, Readmission, and Reoperation After Posterior Lumbar Spinal Fusion: An Analysis of 8492 Patients Using the ACS-NSQIP Database

Michael Brendan Cloney, BA; Roxanna Garcia, MD, MS, MPH; Zachary A. Smith, MD; Nader S. Dahdaleh, MD

Introduction: Corticosteroids have numerous systemic effects. The effects of chronic corticosteroid therapy on rates of complications, readmission, and reoperation after posterior lumbar fusion remains under-investigated.

Methods: We analyzed 8492 patients who underwent posterior lumbar fusion, of whom 353 were steroid users from the National Surgery Quality Improvement Program database (NSQIP) undergoing single and multilevel posterior lumbar spinal fusions. Data was collected on patient demographics, comorbid disease burden, and outcomes. Patients taking steroids for a chronic condition were compared to those who were not. A forward, stepwise, multivariable logistic regression was performed to identify predictors of complications, readmission, and reoperation within 30 days.

Results: Complications were associated with the following: surgery operative time $>240$ minutes (OR 1.66, [1.43-1.93], $\mathrm{p}<0.001$ ), functional status (OR 1.45, [1.05-2.00], $\mathrm{p}=0.022)$, steroid use (OR $1.38,[1.01-1.90], \mathrm{p}=0.044)$, history of COPD (OR 1.36, [1.00-1.84], $\mathrm{p}=0.048)$, diabetes (OR 1.35, [1.12-1.61], $\mathrm{p}=0.001)$, and ASA class (OR 1.34, [1.13-1.58], $\mathrm{p}=0.001$ ). Readmission was associated with the following: ASA class (OR 1.30, [1.04-1.64], $\mathrm{p}=0.022$ ), sepsis after initial surgery (OR 14.72, [8.38-25.85], $\mathrm{p}<0.001)$, superficial surgical site infection (OR 13.58, [8.41-21.93], $\mathrm{p}<0.001$ ), urinary tract infection (OR 2.88, [1.69-4.90], p < 0.001), or DVT/thrombophlebitis after initial surgery (OR 14.36, [8.38-24.62], $\mathrm{p}<0.001$ ). Reoperation was associated with the following: ASA class (OR 1.60, [1.25-2.04], $\mathrm{p}$ $<0.001$ ), sepsis after initial surgery (OR 11.09, [6.71-18.31], $\mathrm{p}<0.001$ ), superficial surgical site infection (OR 11.45, [7.43-17.65], $\mathrm{p}<0.001$ ), initial operative time $>240$ minutes (OR 1.67, [1.31-2.13], $\mathrm{p}<0.001$ ), or DVT/thrombophlebitis after initial surgery (OR 2.35, [1.15-4.80], $\mathrm{p}=0.019)$.

Conclusion: Posterior lumbar spinal fusion for patients on chronic corticosteroid therapy is associated with an increased risk of infectious complications and overall complications, with no associated increase in risk of readmission or reoperation.

\section{The Functional, Histologic and Electrophysiologic Outcomes After Rapid-Stretch Nerve Injury}

Michael Karsy, MD, PhD; Wesley Warner, BS; Stewart Yeoh, PhD; Petr Tvrdik, PhD; Jie Zhang; Mark Alexander Mahan, MD

Introduction: A variety of peripheral nerve injury models have been discussed in the literature, including surgical transection and manual crush methods. However, these injuries do not model stretch injuries, which are common clinical injuries. We hypothesize that a rapid-stretch nerve injury may be a more physiologically relevant injury for approximating clinical challenges and sought to explore the rapid-stretch injury model's reliability and subsequent functional effects.

Methods: We developed a mouse (C57BL/6) model for evaluation of rapid-stretch injury: four injury severity groups were created based on the strain-deformation viscoelastic grade of rapid-stretch injury to the sciatic nerve (sham, elastic, plastic and stretch-rupture). Outcomes were determined by functional tests, including rotarod, taper beam, mouse gait analysis, von Frey filament test and Hargreeves pain assay; histologic analysis of regenerating nerves; and electrodiagnostic studies .

Results: Mice with plastic or stretch-rupture injury showed significantly longer ambulation times, greater foot falls and worse response to Von Frey fibers compared to sham or elastic injury animals. Histology correlated to level of injury and was hallmarked by epineurium and perineurium disruption, as well as neuroma formation. Electrophysiologic data demonstrated reduction in nerve action potential amplitude and decreased compound muscle action potential.

Conclusion: The results of this study support a reliable method for mouse sciatic nerve injury and functional analysis. Functional results and histological changes showed distinct changes depending on the grade of injury. These results also present a model where functional recovery can be monitored and correlated to the histological changes governing nerve regeneration as well as remyelination. Further applications of this model can be in uncovering molecular mechanisms of recovery after peripheral nerve stretch injury. 


\section{Biomechanical Evaluation of Lumbar Decompression Adjacent to Instrumented Segments}

Peter Grunert, MD; Anna Newcomb; Brain Kelly, PhD; Roger Härtl, $M D$

Introduction: Multilevel lumbar stenosis where one level requires stabilization due to spondylolisthesis is routinely treated with multilevel open laminectomy and fusion. We hypothesized that a minimally invasive (MIS) decompression is biomechanically superior to open laminectomy and may allow decompression of the level adjacent the spondylolisthesis without additional fusion. The objective of this study was to study the mechanical effect of various decompression procedures adjacent to instrumented segments in cadaver lumbar spines.

Methods: Conditions tested: 1) L4/L5 instrumentation; 2) L3/L4 minimally invasive decompression; 3 ) addition of partial facetectomy at L3/L4; and 4) addition of laminectomy at L3/L4. Flexibility tests were performed for range of motion (ROM) analysis by applying nonconstraining, pure moment loading $(7.5 \mathrm{nM})$ during flexionextension, lateral bending, and axial rotation. Compression flexion tests were performed for motion distribution analysis.

Results: After instrumentation, MIS decompression increased flexion-extension ROM at L3/L4 by $13 \%(\mathrm{p}=0.034)$ and axial rotation by $23 \% \quad(p=0.003)$. Partial facetectomy further increased axial rotation by $15 \%(\mathrm{p}=0.032)$. After laminectomy, flexion-extension ROM further increased by $12 \%(\mathrm{p}=0.05)$, a $38 \%$ increase from baseline, and axial rotation by $17 \%(\mathrm{p}=0.023)$, a $58 \%$ increase from baseline. MIS decompression yielded no significant increase in segmental contribution of motion at $\mathrm{L} 3 / \mathrm{L} 4$, in contrast to partial facetectomy and laminectomy $(<0.05)$. Fusion led to a signifiant decrease in lumber ROM by about $20 \%$ in all measured planes and to a significant redistribution of motion at all levels $(<0.05)$. Decompression procedures partially restored overall lumbar motion.

Conclusion: MIS decompression is biomechanically far superior to open laminectomy adjacent to instrumented segments. The destabilzing effect of MIS procedures adjacent to fused segments appears to be similar to MIS decompression without adjacent fusion. These results lend support to the concept that in patients undergoing multilevel MIS decompression, fusion might be limited to the segments with actual instability. However great care must be taken to limit facet joint violation in this clinical scenario.

119. Laminoplasty vs. Laminectomy-Fusion for the Treatment of Cervical Myelopathy: Preliminary Results from the CSM-Study Comparing Cervical Sagittal Alignment and Clinical Outcomes

Vijay Ravindra, MD, MSPH; Jill Curran, MS; Praveen V.Mummaneni, MD; Adam S. Kanter, MD; Erica Fay Bisson, MD, MPH; Robert F. Heary, MD; Todd Albert, MD; K. Daniel Riew, MD; Subu N. Magge, $M D$; Robert G. Whitmore, MD; Zoher Ghogawala, MD, FACS

Introduction: Both laminoplasty and laminectomy-fusion effectively treat cervical spondylotic myelopathy (CSM). A direct comparison between them with respect to cervical sagittal alignment and overall health related quality of life outcomes was performed.

Methods: A multi-center prospective, randomized study of CSM is underway. Patients who underwent posterior decompressive surgery with either laminoplasty or laminectomy-fusion were included. Pre-operative and post-operative C2-7 sagittal vertical axis (SVA) and Cobb angle were measured on standing lateral radiographs. Outcomes were assessed with the mJOA scale, NDI, SF-36 PCS and SF-36 MCS, and Euro-QOL-5D. Assessments were performed preoperatively, 6 , and 12 months postoperatively.

Results: A total of 55 patients were available for analysis. There was a significantly greater improvement in the SF-36 PCS (8.3 vs. $3.5, \mathrm{p}=0.03$ ) in the laminoplasty group at 6 months, however the differences in HRQOL outcomes did not persist at one year $(n=35)$. All patients had improvement in mJOA scores and a majority of patients $(>50 \%)$ achieved a minimal clinically important difference (MCID) in each outcome category. A total of 23 patients that underwent laminectomy-fusion $(n=11)$ or laminoplasty $(n=12)$ had one-year clinical and radiographic follow-up. There were no differences in pre-op Cobb angle, C2-7 SVA, or outcome measures. The mean post-operative Cobb angle (12.7 vs. 4.8, $\mathrm{p}=0.27)$ and SVA (23.1 vs. 27.2, $\mathrm{p}=0.44$ ) was similar for both groups, with no significant change from pre-operative measurements; SVA was not a predictor of improvement. Laminoplasty patients developed nonsignificant worse sagittal balance $(+2.8 \mathrm{~mm})$ postoperatively, while laminectomy-fusion patients showed slightly improved balance $(-3.3$ $\mathrm{mm}$ ), not significant.

Conclusion: Both laminoplasty and laminectomy-fusion are effective at treating CSM. At 6 months there was a significantly greater improvement in SF-36 PCS favoring laminoplasty, however at 1 year both groups were comparable. Further studies will define health resource utilization after surgery given differences in healthrelated quality of life at 6 months.

\section{Multidisciplinary Evaluation Leads to the Decreased Utilization of Lumbar Spine Fusion: An Observational Cohort Pilot Study}

Vijay Yanamadala, MD; Jean-Christophe A. Leveque, MD; Quinlan Buchlak; Anna Wright; Yourie Kim; Farrokh Farrokhi, MD; Robert Mecklenburg; Rajiv Sethi, MD

Introduction: Multidisciplinary decision making improves outcomes in many disciplines. The lack of integrated systems for comprehensive care for spinal disorders has contributed to the inappropriate overutilization of spine surgery in the United States.

Methods: We implemented a multidisciplinary conference involving physiatrists, anesthesiologists, pain specialists, neurosurgeons, orthopaedic spine surgeons, physical therapists, and nursing staff. Over seven months, we presented patients being considered for spinal fusion or who had a complex history of prior spinal surgery. We compared the decision to proceed with surgery and the proposed surgical approach proposed by outside surgeons with the consensus of our multidisciplinary conference. We also assessed comprehensive demographics and co-morbidities for the patients and examined outcomes for surgical patients. Statistical significance was $\mathrm{p}<0.05$ using a $\mathrm{X}^{\wedge} 2$-test.

Results: A total of 144 consecutive patients were reviewed at our multidisciplinary conference during the seven month period. Of these, 100 patients had been recommended for lumbar spine fusion by an outside surgeon. Consensus opinion of the multidisciplinary conference advocated for non-operative management in 58 patients $(58 \%)$ who had been previously recommended for spinal fusion by another institution $\left(\mathrm{X}^{\wedge} 2=26.6 ; \mathrm{p}<0.01\right)$. Furthermore, the surgical treatment plan was revised as a product of the conference in $28 \%$ (16) of the patients who ultimately underwent surgery $\left(\mathrm{X}^{\wedge} 2=43.6 ; \mathrm{p}<\right.$ 0.01). We had zero 90-day complications in surgical patients.

Conclusion: Multidisciplinary conferences can significantly influence the care of patients with degenerative spinal disorders, possibly resulting in more appropriate use of surgical interventions and better candidate selection while providing patients with more diverse non-operative treatment options. While long term patient outcomes remain to be determined, such multidisciplinary care will likely be essential to improving the quality and value of spine care. 
121. Comparing the Efficacy of Adipose-Derived vs. Bone Marrow-Derived Stem Cells in Combination with a ClinicalGrade Bone Graft Substitute in a Rat Model of Spinal Fusion

Benjamin D. Elder, MD, PhD; Christina Holmes, PhD; Wataru Ishida, MD; John Locke; Timothy F. Witham, BS, MD

Introduction: Adipose-derived stem cells (ADSCs) have been demonstrated to form vascularized bone in various animal and preclinical models. While bone marrow-derived stem cells (BMSCs) have been widely used in spinal fusion studies, adipose offers a number of advantages as an alternative clinical cell source, including a larger available tissue volume, higher stem cell concentration, and reduced donor site morbidity. In this study we compared the efficacy of ADSCs vs. BMSCs in achieving successful spinal fusion when combined with a clinical-grade bone graft substitute in a rat model.

Methods: ADSCs and BMSCs were isolated from the inguinal fat pads and long bones, respectively, of female Lewis rats (6-10 wk old) and cultured in vitro until passage 2 (P2) for subsequent transplantation. The frequency of colony forming unit fibroblast (CFU-F) colonies was also assessed in vitro for both ADSCs and BMSCs. Posterolateral spinal fusion surgery at L4-5 was performed on 36 female Lewis rats (6-10 wk old) divided into 3 experimental groups: [1] Vitoss (Stryker) clinical-grade bone graft substitute only ( $\mathrm{n}=12)$; [2] Vitoss $+2.5 \times 10^{\wedge} 6 \mathrm{P} 2$ ADSCs /side $(\mathrm{n}=12)$; and [3] Vitoss $+2.5 \times 10^{\wedge} 6 \mathrm{P} 2 \mathrm{BMSCs} /$ side $(\mathrm{n}=12)$. Fusion was assessed at postoperative week 8 via micro-computed tomography (MicroCT) analysis and manual palpation.

Results: ADSCs exhibited a faster proliferative rate and a higher frequency of CFU-F colonies in vitro than BMSCs. The mean fusion volume in the ADSC group was significantly larger than the BMSC and vitoss groups $\left(44.3 \mathrm{~mm}^{\wedge} 3\right.$ vs. 27.6 and $30.0 \mathrm{~mm}^{\wedge} 3$, respectively, $\mathrm{p}<0.01)$. The mean manual palpation score was the highest in the ADSC group compared with the BMSC and VO groups (1.5 versus 0.7 versus $0.8 \mathrm{p}=0.03$ ).

Conclusion: When combined with a bone graft substitute in a rat model, ADSCs yielded increased fusion mass volume and more robust fusion than BMSCs.

122. Allograft Versus Autograft: A Comparison of Postoperative Morbidity in Spinal Fusions Using the ACS-NSQIP Registry

Meghan Murphy, MD; Brandon A. McCutcheon, MD, MPP; Panagiotis Kerezoudis; Patrick R. Maloney, MD; Kendall Snyder, MD; Lorenzo Rinaldo, MD, PhD; Daniel Levi Shepherd, MD; Ahmad Nassr, MD; Mohammed Ali Alvi, MD; Jennifer Grauberger; Mohamad Bydon, MD

Introduction: Autograft for spine arthrodesis has been associated with increased operative times and blood loss. Morbidity of allograft versus autograft use in spinal fusions has not been studied in a large, national multi-institutional cohort.

Methods: The American College of Surgeons' National Surgical Quality Improvement Program (ACS-NSQIP) was queried for patients undergoing cervical or lumbar spinal fusion with either allograft or autograft through a separate incision between 2012 and 2013. The primary outcomes of interest were operative time, length of stay, blood transfusion, and surgical site infection.

Results: A total of 6,790 and 6,718 patients received a cervical or lumbar spinal fusion, respectively. Within the cervical and lumbar cohorts $7.1 \%(n=482)$ and $14.6 \%(n=980)$ received an autograft, respectively. On unadjusted analysis in the cervical cohort, autograft was significantly associated with increased rates of blood transfusion $(2.9 \%$ vs $1.0 \%, \mathrm{p}<0.001)$ and operative time (167 vs 128 minutes, $\mathrm{p}<0.001)$ relative to allograft. In the lumbar cohort, autograft was also significantly associated with increased rates of blood transfusion ( $21.0 \%$ vs $15.7 \%, \mathrm{p}<0.001)$ and operative time (226 vs 204 minutes, $\mathrm{p}<0.001)$. On multivariable analysis in both cohorts, autograft was significantly associated with increased odds of blood transfusion (cervical: OR 2.3, 95\% CI 1.0-5.1 and lumbar: OR 1.3, 95\% CI 1.1 - 1.6) and longer operative times relative to allograft (cervical: 27.8 minutes, 95\% CI 20.7 - 35.0 and lumbar: 25 minutes, 95\% CI 17.7 33.1 ).

Conclusion: In a large national multi-institutional cohort, autograft use was associated with increased rates of blood transfusion and increased operative time relative to allograft.

123. Predictors for Obtaining a Postoperative Improvement Over the Minimum Clinically Important Difference After Lumbar Decompression Surgery

Vincent J. Alentado, MD; Louisa Onyewadume; Patrick Michael Flanigan, BS; Michael P. Steinmetz, MD; Edward C. Benzel, MD; Thomas Mroz

Introduction: Previous studies have reported the importance of obtaining a clinically significant improvement (CSI) after lumbar surgery. However, a comprehensive understanding of preoperative predictors for achieving a CSI after lumbar decompression does not exist.

Methods: The medical records of patients who received a lumbar decompression for any indication were retrospectively reviewed to identify medical and surgical characteristics. A blinded reviewer assessed radiographs for each patient to examine sagittal alignment following decompression. Multivariable logistic regression was used to model the achievement of a CSI based on a EQ-5D MCID value of 0.100 .

Results: A total of 157 patients fit the inclusion criteria; 64 (41\%) exceeded the MCIS value of 0.1001 year postoperatively. Statistically significant independent predictors of not obtaining a CSI included a lower preoperative EQ-5D score $(\mathrm{OR}=37925.3)$ and a higher preoperative PHQ-9 score (OR=1.1). Patients who achieved a CSI at 1 year also had higher EQ-5D Health State and PDQ Functional scores.

Conclusion: This study is the first to use a combination of medical, surgical and postoperative sagittal balance variables as determinants for the achievement of a CSI after lumbar decompression. Lower preoperative quality of life scores and higher preoperative depression scores independently predicted a failure to achieve a CSI 1 year postoperatively. The awareness of these predictors may allow for better patient selection and surgical approach to decrease the probability of acquiring a poor outcome postoperatively.

124. Cervical Total Disc Replacement Using Tissue-Engineered Intervertebral Discs Combined with an Anterior Bio-resorbable Stabilization System in an Ex Vivo Canine Model

Ibrahim Hussain; Gernot Lang, MD; Jorge Mojica Santiago, BS; Rodrigo Navarro-Ramirez, MD, MS; Lawrence J. Bonassar, PhD; Roger Hartl, $M D$

Introduction: Total disc replacement using tissue-engineered intervertebral discs (TE-IVD) offers an alternative biological treatment option for degenerative disc disease. Our group has previously developed a unique TE-IVD that demonstrated efficacy in maintaining disc height, physiological hydration, and tissue integration in beagle models in vivo. However, biomechanical 
properties were inferior to native IVD and implant displacement occurred in several cases. We now investigate the biomechanical responses of our TE-IVDs combined with an anterior bio-resorbable stabilization system (BSS) in an ex vivo canine cervical spine model (Figure 1A-B).

Methods: Using techniques previously described (1,2), TE-IVDs with an inner nucleus pulposus cell-laden alginate layer surrounded by an outer annulus fibrosus cell-laden collagen layer were developed. Cervical spine motion segments $(\mathrm{N}=12)$ of mature beagles were dissected and assessed as intact, after discectomy (Dx), with implanted TE-IVD (IVD-), and with implanted TE-IVD plus BSS $(\mathrm{IVD}+)$ (Figure 2A-C). Using a mechanical testing frame (Figure 2D), unconfined stress relaxation tests were performed. Equilibrium and instantaneous moduli were calculated and normalized to intact motion segments. One-way ANOVA and Tukey HSD were used for statistical analysis, with $\mathrm{p}<=0.05$ considered significant.

Results: Intact motion segments showed equilibrium and instantaneous moduli of $174 \pm 36 \mathrm{kPa}$ and $1760 \pm 430 \mathrm{kPa}$, respectively, with mechanical properties from all other groups significantly lower. After normalizing to their corresponding intact motion segment mechanics, the IVD- group demonstrated relatively similar biomechanical properties to the Dx group, suggesting partial displacement and that low magnitudes of loads are shared by the construct. However, the IVD+ group demonstrated a 2 -fold increase in equilibrium and instantaneous moduli $(p<0.05)$ over the IVDgroup (Figure 3A-B), with no implant displacement observed.

Conclusion: The biomechanical properties of motion segments with BSS increases the stability of TE-IVD constructs and mitigates implant displacement in canine cervical spines ex vivo, providing the impetus for future in vivo studies.

125. Pilot Study Assessing Preservation of Disc Height in an Ovine Model with Polymethyl-Methacrylate and Hyaluronic Acid Injection

Arvin Raj Wali, BA; David Rafael Santiago-Dieppa, MD; Reid Hoshide, MD; Nick Manesis, PhD; Gloria Lin, PhD; William R. Taylor, $M D$

Introduction: Prior histopathologic studies have demonstrated that injection of Polymethyl-Methacrylate-Microspheres (PMMA) into injured inter-vertebral discs may curtail the degenerative and inflammatory processes. The purpose of this study is to assess the radiographic preservation of disc height $(\mathrm{DH})$ over time after insertion of PMMA/Hyaluronic Acid (PMMA/HA) into injured, ovine inter-vertebral discs.

Methods: Six sheepunderwent vertebral disc exposure at 6 contiguous lumbar levels. Two levels served as the injured/treated group receiving PMMA/HA injection, two levels served as the injured/untreated group, and two additional levels served as the uninjured/untreated controls. The sheep tolerated the procedure without complication. Lateral radiographs of the lumbar spine were obtained prior to surgery and at $90 \pm 7$ days $(n=4)$, or at $240 \pm 7$ days $(n=2)$ after injury. R statistical software v3.1.1 was used to perform a one-way ANOVA with Tukey HSD to identify changes in DH among the three groups.

Results: At three months, a $0.912 \mathrm{~mm}(\mathrm{p}=0.291)$ preservation in $\mathrm{DH}$ between the treated and untreated injured discs was observed. Although underpowered, the substantial preservation in $\mathrm{DH}$ at three months demonstrates the potential benefit of PMMA/HA injection. At eight months, there was a small preservation in $\mathrm{DH}$ with a $0.125 \mathrm{~mm}(\mathrm{p}=0.93)$ difference between treated and untreated groups. Subsequent power calculation revealed that a larger study involving 26 vertebrae in both the treated and untreated groups would be required to demonstrate statistical significance at the $95 \% \mathrm{CI}$ at three months.

Conclusion: PMMA/HA injection into an injured intervertebral disc demonstrated statistical trends towards $\mathrm{DH}$ preservation at three months. Although underpowered, this study demonstrates a novel therapeutic approach for DH preservation after injury, and serves as a proof-of-principle for a larger, more powerful study.

\section{Biomechanical Evaluation of the Viscoelastic Cervical Disc Prosthesis in One- and Two-Level Constructs}

Richard D. Guyer, MD; Leonard I. Voronov, MD, PhD; Robert Havey; Gerard Carandang, MS; Saeed Khayatzadeh, PhD; Josh Rubin; Nick Padovani; Kenneth Blank; Stephanie Werner; Avinash Patwardhan, PhD

Introduction: Cervical total disc arthroplasty (TDA) has been proposed as an alternative to prevent adjacent segment degeneration. Optimal placement of a viscoelastic cervical disc (Rhine, K2M Inc.) will replicate natural kinematics. Segmental kinematics will improve with optimal vs anterior placement.

Methods: Seven cadaveric spines (C3-T1) (40.0 \pm 10.2 years) were tested intact, after C5-C6 anterior TDA placement, C5-C6 optimal placement, and C6-C7 optimal placement. Specimens were tested in: flexion-extension with $0 \mathrm{~N}$ and $150 \mathrm{~N}$ compressive preload, lateral bending (LB) and axial rotation (AR). Proper TDA placement was confirmed by fluoroscopy. Load vs. displacement curves were analyzed to determine range of angular motion (ROM) and stiffness.

Results: Optimal C5-C6 TDA placement resulted in no change in flexion-extension ROM compared to intact under $0 \mathrm{~N}$ and $150 \mathrm{~N}$ preload ( $p>0.05)$. LB and AR ROM decreased with TDA $(p<0.01)$. Optimal C6-C7 placement resulted in increased flexion-extension ROM with and without preload $(\mathrm{p}<0.01)$. LB and AR ROM decreased with arthroplasty $(\mathrm{p}<0.01)$. Optimal C5-C6 placement did not change flexion stiffness. The same was true at $\mathrm{C} 6-\mathrm{C} 7$ under no preload, while under $150 \mathrm{~N}$ preload stiffness decreased $(\mathrm{p}<0.01)$. Compared to anterior placement, optimal placement resulted in no significant change in ROM under flexion-extension without preload, LB and AR. Under preload, flexion-extension ROM increased after optimal placement $(\mathrm{p}=0.014)$.

Conclusion: This viscoelastic TDA effectively restored flexionextension motion to intact levels. In LB, TDA maintained $42 \%$ $\mathrm{ROM}$ at $\mathrm{C} 5-\mathrm{C} 6$ and $60 \%$ at $\mathrm{C} 6-\mathrm{C} 7$. In AR, TDA maintained $57 \% \mathrm{ROM}$ at $\mathrm{C} 5-\mathrm{C} 6$ and $70 \%$ at $\mathrm{C} 6-\mathrm{C} 7$. These findings are supported by literature showing cervical TDA results in restoration of approximately $50 \%$ ROM in LB and AR which is a multifactorial phenomenon encompassing TDA design and anatomical constraints. Anterior TDA placement shows motion restoration similar to optimal placement, suggesting its design may be less sensitive to sub-optimal placement.

\section{Can We Increase Segmental Mobility by Cervical} Arthroplasty?

Hsuan-Kan Chang, MD; Jau-Ching Wu, MD, PhD; Chih-Chang Chang, MD; Tsung-Hsi Tu, MD

Introduction: Many reports have unanimously demonstrated that cervical disc arthroplasty (CDA) can preserve range of motion after one- or two-level discectomy. However, little data addressed the amount of changes in segmental mobility after CDA or its clinical correlations.

Methods: Consecutive patients who underwent one-level CDA were retrospectively reviewed. Indications of surgery were medicalintractable degenerative disc disease and spondylosis. Clinical outcomes, including visual analog scale (VAS) of neck and arm pain, neck disability index (NDI), Japanese Orthopedic Association (JOA) scores, were analyzed. Radiographic outcomes, including C2-7 Cobb angle, ?C2-7 Cobb angle (the difference between pre- and post-operative C2-7 Cobb angle), sagittal vertical axis (SVA), ?SVA, 
segmental range of motion (ROM), and ?ROM, were accessed for the association with clinical outcomes. All patients had computed tomography $(\mathrm{CT})$, by which the presence and severity of heterotopic ossification (HO) were thus determined, during the follow up.

Results: A total of 50 patients (mean age $45.6 \pm 9.33$ year-old) of one-level CDA (Prestige LP disc) were analyzed with a mean follow-up of $27.7 \pm 8.76$ months. All clinical outcomes, including VAS, NDI, and JOA scores, improved significantly after surgery. Pre- and post-operative ROM were similar $\left(9.5^{\circ}\right.$ vs $\left.9.0^{\circ}, \mathrm{p}>0.05\right)$ at each indexed level. The mean changes of segmental mobility (?ROM) were $-0.5 \pm 6.13$ degrees. Patients with increased segmental mobility after surgery (?ROM $>0^{\circ}$ ) had less incidences and severity of HO $(\mathrm{p}=0.048)$ than those whose ?ROM $<0^{\circ}$. Segmental mobility (ROM) was significantly smaller in patients with higher $\mathrm{HO}$ grade $(\mathrm{p}=0.012)$, but it did not affect the clinical outcomes. The pre- and post-operative C2-7 Cobb angles and SVA remained similar. The post-operative C2-7 Cobb angles, SVA, ?C2-7 Cobb angles, and ?SVA all had no correlation to clinical outcomes after CDA.

Conclusion: Segmental mobility (mean ROM) and overall cervical alignment (SVA and C2-7 Cobb angle) had no significant differences after one-level CDA. Patients who had increased segmental mobility (?ROM $>0^{\circ}$ ) had significantly less $\mathrm{HO}$ and similarly improved clinical outcomes than those who had decreased segmental mobility $\left(? \mathrm{ROM}<0^{\circ}\right)$.

\section{Fish-mouth Kyphosis of the Device After Cervical Disc Arthroplasty}

Peng-Yuan Herb Chang, MD; Chun-Hao Wang; Tsung-Hsi Tu, MD, Jau-Ching Wu, MD, PhD; Wen-Cheng Huang; Hsuan-Kan Chang, MD; Li-Yu Fay, MD; Ching-Lan Wu; Henrich Cheng

Introduction: There are numerous reports on the safety and efficacy of cervical disc arthroplasty (CDA) in management of 1- or 2-level degenerative disc disease (DDD). However, there is a paucity of data on the complications of CDA, such as kyphotic dislodgement of the disc.

Methods: Retrospective review of consecutive patients who underwent 1- or 2-level cervical disc arthroplasty was conducted. The indications of surgery were myelopathy or radiculopathy or both that were refractory to medical treatment caused by 1- or 2-level DDD or spondylosis. Clinical outcomes were measured in visual analog scale (VAS) for neck and arm pain, Japanese Orthopaedic Association (JOA) scores, and Nurick scales for myelopathy. Radiographic evaluation included post-operative computed tomography or magnetic resonance images and measurement of Cobb's angle of the indexed segments.

Results: One hundred and eight patients (averaged 52 years) were analyzed with a mean follow-up about 2 years. Female to male ratio was $45.13 \%$ vs $54.87 \%$ in this study. $48.67 \%$ of patients underwent 1-level and $51.33 \%$ of patients underwent 2-level surgery. Among the total of 171 disc levels treated, C5-6 (52.05\%) was the most common segment. During the follow up, two $(1.8 \%)$ patients developed fishmouth kyphosis of the device post-operation. The first patient had a single-level CDA at C3-4, which then dislodged within 6 months of post-operative follow up. The severely kyphotic and migrated device prompted revision surgery to an instrumented anterior cervical discectomy and fusion. The second problematic patient had 2-level CDA at C3-4-5. During the first year of follow up, both discs had subsidence and became kyphotic but caused little symptoms. The second patient remained asymptomatic to date, over 24 months, and thus no secondary surgery yet. Both patients' myelo-radiculopathy got improved after the first CDA operation. Despite the above two drastic complications, patients of the current series had overall improvement in every clinical outcome measurement.

Conclusion: Although uncommon (1.8\%), problematic subsidence and kyphotic dislodgement of the device could occur after CDA surgery. Revision with anterior cervical discectomy and fusion might be a good salvage for symptomatic patients.

130. Cervical Total Disc Replacement Versus Anterior Cervical Discectomy and Fusion: Reoperation Rates, Complications, and Hospital Resource Utilization in 72,688 Patients in the United States

\section{Kyle Anthony Smith, MD; Kavelin Rumalla; Paul M. Arnold, MD}

Introduction: Healthcare readmissions are an important cause of increased cost, have profound clinical impact, and are directly linked to reimbursement. The study of 30-day readmissions in spine surgery has been well documented. However, in cases of anterior spine surgery for degenerative conditions, the rates, causes, risk factors, costs, and outcomes of readmissions are not well understood outside the 30-day time period.

Methods: The Nationwide Readmissions Database is a national source by the Agency for Healthcare Research and Quality approximating $50 \%$ of all U.S. hospitalizations with patient identifiers to track patients longitudinally. Patients greater than 18 years old were identified. Patient demographics, comorbidities, pre-operative characteristics, and surgical characteristics were chosen for predictor variables. Rates of readmission for 30-days and 90-days were calculated. Complications, outcomes, and costs were also analyzed using SPSS v.23 via univariate and multivariable analyses.

Results: Between January and September 2013 a total of 72,688 patients were identified. The 30-day and 90-day readmission rates were $2.67 \%$ and $5.97 \%$, respectively. The most prevalent reason for 30-day readmission was complication of medical or surgical care $(20.3 \%)$ whereas for 90 -day readmission, degenerative spine etiology (19.2\%). Common risk factors for 30- and 90-day readmission included older age, male gender, Medicare/Medicaid payer status, prolonged initial length of stay (LOS), and various comorbidities. Unique risk factors for 30-day readmissions included adverse discharge disposition and for 90-day readmissions included mechanical implant-related complications. When comparing ACDF and TDR, ACDFs were associated with an increased rate of 90-day readmission $(6.0 \%$ vs. $4.3 \%)$ in univariate but not multivariable analysis. Additionally, the TDR cohort had a shorter LOS, lower complication rate, and fewer adverse discharge dispositions.

Conclusion: Identification of causes and predictor variables for readmission following anterior cervical spine surgery is important to potentially allow for changes in peri-operative management. Decreasing readmissions would improve patient outcomes and reduce healthcare costs while increasing reimbursement.

132. Cervical Arthroplasty Versus Anterior Cervical Fusion for Symptomatic Adjacent Segment Disease After Anterior Cervical Fusion Surgery: Review of Treatment in 41 patients

\section{Sang-Bok Lee, MD, Kyoung-Suok Cho, MD, PhD}

Introduction: The purpose of this study is to evaluate the efficacy and safety of cervical total disc replacement (TDR) for symptomatic adjacent segment degeneration (ASD) with previous anterior cervical discectomy and fusion (ACDF) was done, compared to ACDF in the treatment of cervical ASD.

Methods: Between 2010 and 2014, 41 patients with previous cervical fusion surgery underwent ACDF orTDR for symptomatic ASD. Twenty two patients underwent twenty four ACDFs and 19 patients underwent twenty five arthroplasties of the Prodisc- $\mathrm{C}$ and Active-C for symptomatic ASD. Clinical outcome was measure by visual analogue scale (VAS) for arm, neck disability index 
(NDI) and Odom's criteria. Radiological evaluation was performed preoperatively and postoperatively to check the change of the angular range of motion (ROM) for cervical spine and adjacent segments. The radiological change of adjacent segments degeneration (ASD) was also assessed.

Results: The C-TDR group showed better NDI improvement after surgery $(\mathrm{P}<0.05)$. Clinical outcomes using VAS on arm pain and Odom's criteria were significantly improved in both groups. The mean C2-7 ROM of C-TDR group showed faster recovery than that of ACDF group and maintained the preoperative values during the follow up periods. However, that of ACDF group did not recovery preoperative values $(\mathrm{P}>0.05)$. There was significant difference of the inferior adjacent segment ROM between C-TDR and ACDF group $(\mathrm{P}<0.05)$. The ACDF group had a higher incidence of radiological changes in adjacent segment compared with $\mathrm{C}$-TDR group $(\mathrm{P}<0.05)$.

Conclusion: ACDF and C-TDR for symptomatic ASD seem to be effective to reduce the arm pain and recover the functional outcomes. C-TDR group was better than the ACDF group in terms of NDI and incidence of adjacent segment degeneration. Analysis of radiological outcome, C-TDR showed better C2-7 ROM recovery and less ROM of inferior adjacent segment increase.

133. Cervical Arthroplasty for Moderate to Severe Disc Degeneration: Clinical and Radiological Assessments After a Minimum Follow-Up of 24 Months

\section{Seung Hwan Yoon}

Introduction: Clinical outcomes and radiologic results after cervical arthroplasty have been reported in many articles, yet relatively few studies after cervical arthroplasty have been conducted in severe degenerative cervical disc disease.

Methods: Sixty patients who underwent cervical arthroplasty (Mobi-C®) between April 2006 and November 2011 with a minimum follow-up of 18 months were enrolled in this study. Patients were divided into two groups according to Pfirrmann classification on preoperative cervical MR images: group A (Pfirrmann disc grade III, $\mathrm{n}=38$ ) and group $\mathrm{B}$ (Pfirrmann disc grades IV or $\mathrm{V}, \mathrm{n}=22$ ). Visual analogue scale (VAS) scores of neck and arm pain, modified Oswestry Disability Index (mODI) score, and radiological results including cervical range of motion (ROM) were assessed before and after surgery.

Results: VAS and mean mODI scores decreased after surgery from 5.1 and 57.6 to 2.7 and 31.5 in group A and from 6.1 and 59.9 to 3.7 and 38.4 in group B, respectively. In both groups, VAS and mODI scores significantly improved postoperatively $(p<0.001)$, although no significant intergroup differences were found. Also, cervical dynamic ROM was preserved or gradually improved up to 18 months after cervical arthroplasty in both groups. Global, segmental and adjacent ROM was similar for both groups during follow-up. No cases of device subsidence or extrusion were recorded.

Conclusion: Clinical and radiological results following cervical arthroplasty in patients with severe degenerative cervical disc disease were no different from those in patients with mild degenerative cervical disc disease after 24 months of follow-up.

\section{Minimally Invasive Spine Surgery in the Pediatric Population: A Case Series}

Richard P. Menger, MD, MPA; Matthew Hefner, MD; Anthony H. Sin, $M D$

Introduction: Minimally invasive spinal surgery (MIS) has historically been applied to the adult population. Almost no literature reports can be found regarding routine application of
MIS techniques to spinal pathology in the pediatric population. However, due to its minimal disruption, MIS shows great promise in appropriately selected pediatric patients. Here we illustrate the patient characteristics, operative technique, and surgical outcomes for minimally invasive lumbar procedures at our institution.

Methods: Consecutive pediatric patients undergoing elective MIS lumbar spine procedures were retrospectively analyzed from a single fellowship trained academic spinal neurosurgeon from 2008 to 2016. Information was retrieved regarding procedure and disease pathology. Descriptive data was obtained including age, sex, body mass index, insurance coverage, smoking status, and co-morbidities. Outcome measures were recorded including intraoperative complications, revision surgery, and return to function.

Results: 16 individual patients underwent 17 procedures. The median BMI was 29.2. The range of BMI scaled from 20.8 to 41.5 . Ages ranged from $12-19$. Nearly $20 \%$ of the pediatric patients in our series were smokers. Most patients underwent discectomy, with L5-S1 being the most common level. One patient underwent direct pars defect repair, and another underwent recurrent discectomy. Nearly $90 \%$ of patients were complication free. One patient had a recurrent disc herniation, and another had a superficial wound infection. $82.4 \%$ patients enjoyed a full return to sports such as weight lifting, gymnastics, and contact sports. One patient required pain management to help alleviate ongoing pain. Another patient required a course of outpatient rehab to help with a foot drop pathology.

Conclusion: Advances in MIS surgery allow for application to a wider population. Our series illustrates the effective application of MIS techniques to carefully selected pediatric patients. Emphasis is on minimal disruption. The average patient in our series was overweight and nearly $20 \%$ of the patients were smokers.

\section{Economic Performance of Oblique Lateral Lumbar Interbody Fusion (OLLIF) with a Focus on Hospital Throughput Efficiency}

\section{Hamid R. Abbasi, MD, PHD, FACS; Christopher Murphy}

Introduction: Oblique lateral lumbar interbody fusion (OLLIF) is a minimally invasive lumbar surgery. Differences in resource consumption between open spinal surgeries, transformational lumbar interbody fusions (TLIF) and OLLIF, are not documented. We monetize quantifiable differences in resource utilization between the two procedures. A retrospective review of 124 surgeries was performed $(\mathrm{OLLIF}=69, \mathrm{TLIF}=55)$. Standard conversion factors were used and values reported based on the levels (1-4) addressed at surgery. Per case, reduced resource consumption suggests lower total hospital costs. Reduced surgical time and LOS can result in greater patient throughput per operating room and patient bed for OLLIF patients in hospitals that have resourced constrained environments.

Methods: This was a retrospective case series including 69 OLLIF patients and 55 open TLIF controls. The exempt status of this study, in accordance with FDA was approved by the Pearl Institutional Review Board in February 2015.

Results: One level surgery time (OLLIF 62.9 vs. TLIF 134.9 minutes) and surgical expense (OLLIF \$5,253 vs. TLIF \$11,264) were reduced in the OLLIF population. Inpatient costs (OLLIF \$5,712 vs. TLIF \$9,271) and length of stay (LOS) were also reduced (OLLIF 2.6 vs. TLIF 4.2 days). There were no significant differences between the groups in either BMI or age. The only exception was that OLLIF three level patients were significantly older than their counterparts in the TLIF comparison group.

Conclusion: The cost reductions and faster recovery times associated with the OLLIF procedure make it an appealing alternative to the traditional open fusions available for patient and insurance providers. The reduction in the use of these key hospital resources suggests that hospitals that are constrained by OR or hospital bed availability may be able to achieve greater throughput efficiency by 
increasing the overall percentage of patients receiving the OLLIF surgery.

\section{Lateral Lumbar Interbody Fusion with Lateral Modular Plate Fixation and Interspinous Process Fixation: 2-Year Outcomes from a Prospective Multi-Center Study}

Clint Hill; Brandon Strenge; Chris M. Ferry, BS, MS; Brieta Bejin; Sarah Martineck; Kim Martin; Tom Glorioso, MS; Ryan Peter Denhaese, $M D, M S$

Introduction: Lateral lumber interbody fusion (LLIF) with integrated lateral modular plate fixation (MPF)(Fig. 1) can be an advantageous technique when seeking to enhance anterior column rigidity and diminish the need for extensive posterior fixation. The aim of this analysis was to determine 1) whether MPF increases intraoperative demand and 2) whether outcomes of LLIF+MPF, supplemented with interspinous process fixation (ISPF), are comparable to those of traditional LLIF with adjunctive pedicle screw fixation (PSF).

Methods: Data was retrospectively collected from a prospective multi-center study. Eight LLIF+MPF+ISPF subjects were compared with 15 LLIF+ISPF subjects whom received intervention during the same time period of the study. Posterior fixation was determined by parent study randomization (2:1 ISPF to PSF subjects). All cases were single-level for the treatment of symptomatic degenerative disc disease and/or spondylolisthesis (= Grade 2). Perioperative, patient reported, and radiographic outcomes, as well complication profiles, were reported at $1.5,3,6,12$, and $24 \mathrm{mos}$.

Results: ISPF subjects experienced significantly less blood loss, OR time, incision lengths, and fluoroscopy than PSF subjects in the posterior aspect. No intraoperative or perioperative procedural related complications were observed in the MPF+ISPF cohort. Mean ODI score change from baseline at $12 / 24$ mos post-op was 25.7/22.5 for LLIF+MPF+ISPF subjects and 20.4/23.5 for PSF subjects, respectively (Fig. 2). Mean ZCQ Physical/Symptom and SF-36 Physical/Mental scores were comparable at $24 \mathrm{mos} .100 \%$ of LLIF+MPF+ISPF subjects and 78\% of LLIF+PSF subjects achieved solid interbody fusion.

Conclusion: LLIF+MPF+ISPF demonstrated clinically advantageous outcomes out to $24 \mathrm{mos}$, comparing well to LLIF+PSF, the standard-of-care for circumferential LLIF.

137. Lumbar Plexus Injury After MIS Lateral Transpsoas Interbody Fusion at L4/5 Level: A Review of 62 Consecutive Cases

Jacob Januszewski, DO; Shashank Gandhi; Konrad Bach, MD; Andrew C. Vivas, MD; Chun Po Yen; Jason Michael Paluzzi; Juan S. Uribe, $M D$

Introduction: The MIS lateral transpsoas approach (MIS-LIF) has become an increasingly popular means of fusion. Its most frequent complication is lumbar plexus injury. Reported complication rates at the L4-5 disc space vary widely in the literature, bringing into question the safety of MIS-LIF for the L4-5 region. Single level complication rates have been rarely reported alone. We report our experience with lumbar plexus injuries, safety, and efficacy of MISLIF at the L4-5 disc space.

Methods: A retrospective analysis of our MIS-LIF database was performed from 2011-2016. All patients with a standalone or supplemental lateral plate or posterior percutaneous pedicle screw MIS-LIF at the L4/5 level were included. Patients with multilevel fusions were excluded. We analyzed lumbar plexus injuries and the date of resolution.
Results: Total of 62 patients of 303 met inclusion criteria. Twelve $(19 \%)$ had an immediate postoperative complication, and almost all were transient and sensory. One had a delayed contralateral sensory and motor $(2 / 5)$ femoral nerve injury from psoas hematoma. All patient complications except for 2 have completely resolved by the 12-month follow-up resulting in long-term complication rate of $3.2 \%$. The mean LOS and follow-up were 2.1 days and 15 months, respectively. The average ODI improved from 60 to 37 at the last follow-up. The VAS score improved from 7 to 3 at the last followup. There were no reoperations secondary to hardware failure or symptomatic pseudarthrosis.

Conclusion: MIS-transpoas LIF at the L4-L5 disc space is a safe, reproducible, and effective technique with low rate of lumbar plexus injury. When careful surgical technique is applied, patient outcomes may be better than in multilevel MIS-LIF operations above the L4-L5. MIS-LIF at L4/5 also offers lower intraoperative and wound complications than other techniques for this level. Most complications that do occur are transient neuropraxia or mild axonotmesis, which resolve within 6 months.

138. Comparative Study of Intraoperative True CT Radiological Outcomes After Minimally Invasive Extreme Lateral Interbody Fusion (ELIF) and Minimally Invasive Transforaminal Lumbar Interbody Fusion (TLIF)

Rodrigo Navarro-Ramirez, MD, MS; Gernot Lang, MD; Insa Janssen; Ajit Jada; Connor Berlin; Aaron Hilis; Micaella Zubkov Weill, BS; Roger Hartl

Introduction: To date there are still questions unanswered regarding the factors associated to a better radiological outcome after direct (MIS-TLIF) vs indirect decompression (ELIF). The purpose of the study was to intraoperatively assess and evaluate the degree of neural decompression through minimally invasive MIS-ELIF and MIS-TLIF utilizing a novel intraoperative CT (iCT).

Methods: A retrospective single-center study was conducted. Radiological parameters using a true intraoperative CT scanner were recorded from 34 patients (41 levels) undergoing MIS-ELIF (18 patients, 22 levels) and MIS-TLIF (16 patients, 19 levels). The indication for surgery was spinal stenosis with or whithout GI-GII degenerative scoliosis. Only patients with pre and immediate postoperative spine scans were included. Disc height (DH), Foraminal area (FA), Canal surface area (CSA), Facet tropism (FT), Facet joint degeneration (FD), Segmental disc angle (SDA) and Lumbar lordosis (LL) were analysed pre- and postoperatively.

Results: 34 patients containing 41 spinal motion segments were analysed. Intraoperative CT-based navigation was successfully accomplished in all patients and the quality was sufficient for morphological analysis of all spinal segments. Radiographic parameters, including disc height $(?=+4.3 \mathrm{~mm} /+2.8 \mathrm{~mm})$, axial central canal surface area $\left(?=+57.1 \mathrm{~mm}^{2} /+102.4 \mathrm{~mm}^{2}\right)$, foraminal area $\left(?=+42.3 \mathrm{~mm}^{2} /+37.1 \mathrm{~mm}^{2}\right)$, segmental disc angle $(?=+7.32 \%$ $\left.+6.11^{\circ}\right)$ and lumbar lordosis $\left(?=+9.64^{\circ} / 3.3^{\circ}\right)$ revealed significant improvement immediately after MIS-ELIF and MIS-TLIF surgeries $(p=0.05)$. Despite a significantly stronger restoration of disc height in MIS-ELIF compared to MIS-TLIF $(p=0.05)$ no significant increases in the foraminal area, the central canal surface and the lumbar lordosis were demonstrated between MIS-ELIF and MIS-TLIF $(p=0.05)$. Facet degeneration did not affect the amount of neural decompression in MIS-ELIF.

Conclusion: Intraoperative CT was capable of assessing neural decompression by MIS-ELIF and MIS-TLIF with high accuracy. Both procedures demonstrated equivalent efficiency in neural decompression and restoration of spinal biomechanics. 
139. Minimally Invasive Transforaminal Vs. Direct Lumbar Interbody Fusion: Effect on Return to Work and Narcotic Usage

Terence Verla, MD, MPH; Rory R. Mayer, MD; Lona Winnegan, NP-C; Ibrahim Omeis, MD

Introduction: Direct (DLIF) and transforaminal (TLIF) lumbar interbody fusions produce satisfactory clinical outcomes with significant reduction in pain and functional disability. Despite the increasing utilization in complex spinal deformity surgeries, there is paucity of data comparing outcome measures of minimally invasive (MIS) TLIF and DLIF. The purpose of this study was to assess and compare time to quality of life, return to work and narcotic usage in patients undergoing one level DLIF and TLIF

Methods: This is a retrospective study including patients who underwent MIS, one level, TLIF or DLIF between 2013 and 2015. Clinical outcome measures such time to return-to-work, narcotic requirement, VAS and ODI were compared between cohorts. We excluded patients with previous same level fusion.

Results: 57 patients were included (DLIF-17pts; TLIF-40pts). Average age: $56.1+/-9.9 y$ rs (DLIF) and 59.9+/-12.5yrs (TLIF). Preoperatively, there was no difference in VAS pain score (DLIF: 8.6+/-1.5; TLIF: $7.9+/-1.7, \mathrm{p}=0.14$ ), or ODI (DLIF: $50.25+/-2.76$; TLIF: $51.41+/-2.08 ; \mathrm{p}=0.73$ ). Average follow-up was $8.1+/-3.8$ months (DLIF) and $6.7+/-4.9$ months (TLIF), $\mathrm{p}=0.33$. Overall, there was a significant improvement in post-operative VAS score and ODI. When compared, there was no significant difference in the post-operative VAS scores between both cohorts (DLIF: 3.7/- 2.4 vs $2.60+/-2.6 ; \mathrm{p}=0.31$ ), and also the ODI (DLIF: 23.12+/-2.91; TLIF: $26.71+/-1.99 ; \mathrm{p}=0.30$ ). The duration of post-operative narcotic use was similar in both cohorts (DLIF: $4.8+/-4.7$ months vs TLIF: $5.2+/-5.1$ months, $\mathrm{p}=0.82$ ). There was a significantly longer time to return to work in TLIF $(7.1+/-4.8$ months) compared to DLIF patients $(2.3+/-1.3, \mathrm{p}=0.006)$.

Conclusion: Both MIS TLIF and DLIF provide long-term improvement in pain and functional outcomes, with an overall reduction in post-operative narcotic requirement. This study shows pain improvement, hospital length of stay, and duration of narcotic usage post-operatively are similar in both groups. However, DLIF patients returned to work significantly sooner.

140. Management of Lumbar Adjacent Segment Disease (ASD) with Lateral Lumbar Interbody Fusion

Karthik Madhavan, MD; Zachary Stephen Hubbard, BS; Lynn McGrath, Jr., MD; Lee Onn Chieng, BS; Christoph Paul Hofstetter, $M D, P h D ;$ Seth K. Williams, MD; Steven Vanni, DO

Introduction: Adjacent segment disease is a known complication of lumbar spine fusion (6-39\%) and requires additional fusion procedure. However, dissection through a previous scar entails the risk of dural tear and poor wound healing for posterior approach. Anterior approach is not feasible at all levels. Lateral lumbar interbody fusion (LLIF) has gained popularity for its minimally invasive approach and has gradually expanded its indications. We present largest case series till date on management of ASD with exclusive LLIF.

Methods: We performed a retrospective chart analysis of all the patients who underwent lateral interbody fusion (LLIF) since 2006 and identified 55 patients specifically treated with LLIF for adjacent level disease. We evaluated the outcome based on the segmental and regional lumbar lordosis. Statistics were done using SPSS 22.

Results: In our 55 patients, average age was $63.0 \pm 9.7$ years old with mean follow up of $13.7 \pm 17.0$ months. The average blood loss was $116.3 \mathrm{ml}$ (range, $5-500 \mathrm{ml}$ ). $42 \%$ of the patients were obese and $12 \%$ were smoker. There was significant intervertebral disc height restoration from a mean preoperative value of $8.6 \pm 2.6 \mathrm{~mm}$ to $12.3 \pm$ $2.2 \mathrm{~mm}$ at last follow up $(\mathrm{p}<0.001)$. There was significant reduction of
VAS value of low back by $61.9 \%(\mathrm{p}=0.004)$ and leg pain by $55 \%$ but latter was not statistically significant. With regards to complications, 1 patient had subsidence and 3 had pseudarthrosis. 6 patients developed radiological ASD during follow up but one required fusion for symptomatic disease.

Conclusion: Although there is ligament and muscle preservation, we still encountered six radiological ASD with only one requiring fusion which is better than literature. There was significant improvement in VAS scores from pre-operative status. There is good disc height restoration and improvement in leg pain which eventually translates to better patient satisfaction. LLIF is a feasible alternative to manage ASD.

141. Reduction of Postoperative Narcotic Consumption in Awake TLIF: Enhanced Recovery After Surgery

Karthik Madhavan, MD; Hsuan-Kan Chang, MD; Timur Urakov, MD; Lee Onn Chieng; Anand Veeravagu, MD; Michael Y. Wang $M D, F A C S$

Introduction: TLIF is a minimally invasive procedure, which claim to reduce blood loss, length of stay and narcotic consumption. Although the procedure causes minimal disruption of the tissue the body still reacts in a similar fashion as conventional open approach with stress from metabolic changes and anesthesia. In our institution, as part of the enhanced recovery after surgery (ERAS) protocol, we performed awake TLIFs and present our data on postoperative narcotic consumption.

Methods: We performed a retrospective chart review of 50 patients which included the first 24 patients done awake and the other 26 patients done in a traditional intubated fashion. We evaluated the daily consumption of narcotics beginning from the day of surgery till discharge.

Results: Our analysis included patients who underwent awake TLIF $(n=24)$ and MIS TLIF $(n=26)$. 2-level fusions were performed on two patients in awake TLIF and one in traditional TLIF group while the rest underwent single level fusion. The average age was 61.5 \pm 12.3 years old. All the awake patients received 72-hour long acting depoform bupivacaine (Exparel, Pacira.inc) intraoperatively at the beginning of the case. The narcotic consumption was significantly lower in the awake group from postoperative day 0 to 5 compared to the traditional MIS TLIF group. In addition the length of stay was significantly less in awake patients.

Conclusion: TLIF is quite a sophiscated surgery in itself but body reacts with significant metabolic and anesthetic stress. Efforts in utilizing measures to avoid intubation, optimal perioperative fluid, and long acting pain relief have further reduced postoperative pain and improved patient satisfaction. This makes awake TLIF with fast Track recovery even more atttractive .

142. Does Minimally Invasive Transforaminal Lumbar Interbody Fusion Influence Sagittal Spinopelvic Parameters in Single-level Low-grade Isthmic Spondylolisthesis? Comparative Analysis with Conventional Technique

Dal-Sung Ryu; Sang-Soak Ahn; Kyung-Hyun Kim; Jeong-Yoon Park; Sung-Uk Kuh; Dong-Kyu Chin; Keun-Su Kim; Yong-Eun Cho

Introduction: To compare the clinical and radiographic results between open transforaminal interbody fusion (TLIF-O) and minimally invasive TLIF (TLIF-M) for single-level low grade isthmic spondylolisthesis (IS). We had particular focus on the sagittal alignment parameters, pelvic parameters, and slip parameters.

Methods: This study enrolled 45 patients who underwent singlelevel TLIF who were diagnosed with low grade IS and were 
followed up for at least one year with plain radiography, and CT. The patients were divided into two groups according to operative method: TLIF-M (20 patients) and TLIF-O (25 patients). TLIF-O were grouped into matched with TLIF-M. The primary outcome measures were the sagittal alignment parameters (sagittal vertical axis [SVA], lumbar lordosis [LL], and segmental lordosis [SL]), pelvic parameters (pelvic incidence [PI], pelvic tilt [PT], and sacral slope [SS]), and slip parameters (height of the intervertebral disc [HOD], slip degree [SD], and slip angle [SA]). Secondary outcome measures included fusion rates, clinical outcomes assessed with visual analog scale (VAS) and Oswestry Disability Index (ODI), and perioperative outcomes assessed with blood loss, operation time, hospital stay and complications.

Results: At one-year postoperatively, there were no significant differences in any radiologic parameters between two groups. In intragroup comparison, SD and HOD were significantly improved in TLIF-M. SVA, PT, SD, SA, and HOD were significantly improved in TLIF-O. Perioperative results such as blood loss, operation time, and hospital stay were superior in TLIF-M than TLIF-O Fusion was achieved in 17 of 20 patients (85.0\%) in TLIF-M and 23 of 25 $(92.0 \%)$ patients in TLIF-O. Back VAS and ODI scores of TLIF-O revealedsignificantly lower than TLIF-M at one (back VAS, ODI) and 6 month (ODI) postoperatively.

Conclusion: TLIF-M and TLIF-O produced similar clinical and radiological outcomes, including reduction of spondylolisthesis and disc space height restoration at one year follow-up for singlelevel low grade IS. Although TLIF-O showed a trend toward more improvement in SVA, PT, and SA in terms of radiologic findings, radiologic parameters were physiologic ranges in both groups.

143. Does the Posterior Lumbar Interbody Fusion(PLIF) With Percutaneous Pedicle Screw Fixation(PPS) at Lumbosacral Spine Have Good Results Comparing with it's Use at Lumbar Spine?

Sang-Don Kim; Hyun-Jin Hong

Introduction: Percutaneous pedicle screw (PPS) fixation with fluoroscopic guidance is one of the most common techniques of minimal invasive surgery (MIS) now these days. Despite advantages of PPS as a minimal exposure is well known, pedicle screw insertion at L5-S1 lesions can be problematic compared to other lumbar areas because of its different anatomical structure such as the shape of spinal canal, angulation of pedicle and track of medial branch nerves. The purpose of this study is to evaluate clinical outcome of PLIF with PPS between lumbar and lumboracral spine.

Methods: A total of 214 patients were operated using PLIF with PPS at a single center by single surgeon between January 2005 and December 2015. Among them, 144 cases (59 male, 149 female) of one level PLIF with PPS were reviewed retrospectively and minimal follow-up period is one year. The levels operated were $\mathrm{L} 3-4(\mathrm{~N}=10)$, $\mathrm{L} 4-5(\mathrm{~N}=88)$ and L5-S1 $(\mathrm{N}=46)$. Operation time, amount of blood loss and postoperative drainage were analyzed between each three groups and clinical outcomes were also examined by visual analogue scale (VAS) for preoperative day, immediate postoperative day, postoperative 1, 2, 6 month and 1 year. One-way ANOVA tests were used for data analysis $(\mathrm{p}<0.05)$.

Results: There was no significant difference to age (mean: 60.49), gender (Female to male: 1.6:1), operative time (mean: 210.15minutes), amount of blood loss (mean: $315.03 \mathrm{ml}$ ) and postoperative drainage (mean: $236.44 \mathrm{ml}$ ) between each three groups. Patients who had L4-L5 lesion were found to have more preoperative pain than L5-S1 lesion $(\mathrm{p}=0.002)$, so the VAS for post-operative 1 month was 3.28 for L5-S1, 3.78 for L3-L4 and 4.28 for L4-L5 with a statistically significant difference $(\mathrm{p}<0.001)$. Conversely, the VAS for post-operative 2 month was 4.10 for L5-S1, 2.83 for L3-L4 and 3.05 for L4-L $5(\mathrm{p}<0.005)$. Finally, the difference of VAS between preoperative and postoperative 1 year follow-up was 3.86 for L5-S1,
5.5 for L3-L4 with significant difference $(\mathrm{p}=0.035)$, but 3.96 for L4-L5 without significant difference $(\mathrm{p}=0.194)$.

Conclusion: Comparing the degree of clinical improvement, PLIF with PPS at lumbosacral junction is tended to be less than other lumbar spine (L3-L4 with significant difference). So, surgeons must consider the anatomical configuration and more careful procedures at L5-S1 level when operation is performed at lumbosacral junction.

\section{Open vs. Minimally Invasive TLIF: Review of Outcomes and Consideration for Patient Selection}

Liesl Close; Patrick W. Hitchon, MD; Jennifer Noeller, ARNP; Naalia Hramakova

Introduction: Minimally invasive spine surgery (MIS), using a dilating tube and microscope to achieve transforaminal interbody fusion (TLIF), has been advocated to hold an advantage when compared to conventional open surgery. For verification, we retrospectively reviewed our TLIF patients who underwent surgery via open or MIS technique.

Methods: Patient cohorts were retrospectively reviewed. Age, body mass index, number of levels fused, duration of surgery, estimated blood loss, length of hospital stay, and complications were reviewed.

Results: 31 patients underwent MIS TLIF. Patients had a mean (+/- SD) age of $64+/-12$, and BMI of $28+/-7$ underwent TLIF at an average of $1.1+/-0.4$ levels. Six of these patients had undergone previous lumbar back surgery. The duration of surgery was $252+/-83$ minutes, and a blood loss of $156+/-151 \mathrm{ml}$. Length of hospital stay was $4+/-2$ days, and follow-up was at an average of 58 months. One patient suffered a wound infection, necessitating debridement. Visual analog score for pain (VAS) and Oswestry disability index were $1.2+/-1.7$ and $27+/-22$ respectively. Seventy three patients with ages of $62+/-13$ and BMI of $30+/-6$ underwent Open TLIF for an average of 1.4+/-0.6 levels. Thirty eight of this cohort or nearly $50 \%$ had undergone previous lumbar surgery. Duration of surgery and blood loss was $300+/-87$ minutes and $427+/-417 \mathrm{ml}$ respectively. Hospital stay was $4.6+/-2.8$ days, and follow-up was at an average 149 months. Three patients of this cohort suffered an infection, and one required hardware revision. At follow-up VAS and Oswestry impairment score were $2.3+/-3.0$ and $30+/-19$.

Conclusion: Patients most suited for MIS surgery had not undergone previous lumbar spinal surgery. Open surgery is favored for revision surgery particularly where extension of fusion and instrumentation are necessary. Duration of surgery, blood loss, number of levels, and hospital stay were longer with Open compared to MIS surgery. At follow-up, VAS and Oswestry scores were not significantly different. The selection for MIS or open surgery should take into account any history of previous surgery, and the need for augmentation of implanted hardware.

145. Oswestry Disability Index (ODI), Safety and Fusion Rates in Minimally Invasive Transforaminal Lumbar Interbody Fusion (MITLIF): Multi-Center Prospective Longitudinal Cohort Study

Troy Dawley, DO; Justin G. Thomas, DO; Liisa Bergmann, MD; Doris Tong, MD; Teck Mun Soo

Introduction: MITLIF is becoming more prevalent. Outcomes for multi-level MITLIF are inadequately studied. We seek to demonstrate the safety and efficacy of multi-level MITLIF regarding ODI and fusion outcomes.

Methods: We screened all consecutive patients who underwent minimally invasive multi-level lumbar interbody fusion in 2012 at 2 hospitals by multiple surgeons. We excluded patients who were 
less than 18 years, non-compliant with follow up, or had auto/ workers' compensation. The primary outcome was fusion rate with $>6 \mathrm{~m}$ postop radiographic (XR) follow up. Secondary outcomes were ODI, perioperative complications, blood transfusions, and duration of surgery. We prospectively conducted ODI at $12 \mathrm{~m}$ postop through office visit or phone interview. Four view postop lumbar XR were evaluated by an independent neuroradiologist. Fusion criteria consisted of absence of movement on flexion/extension films, interspace trabeculation formation and degree of hardware lucency. The perioperative complications were collected by chart review and patient survey at one month follow up. Descriptive statistics were used.

Results: We studied 221 interspaces in 116 patients, and patient demographics are in Table 1. Our fusion/interspace was $91.3 \%$ at a mean follow-up of 12.4 months ( +5.2 , Table 3$)$. The mean preop ODI was $39.8 \pm 18.5$; mean $12 \mathrm{~m}$ ODI was $30.1 \pm 20.2$; mean ODI difference preop vs. $12 \mathrm{~m}$ was $10.6 \pm 19$ (Table 2 ). The mean operative duration was $2.46 \mathrm{~h} \pm 0.81$. Perioperative complications were limited to $1.74 \%$ $(n=2$, Table 4$)$ of patients which consisted of one epidural hematoma requiring evacuation and one pulmonary embolism within one month of surgery.

Conclusion: We found that multi-level MITLIF could be performed with acceptable fusion rates, patient functional outcomes and complication rates. The next step will be a controlled trial or observational studies with a larger population. Studies with a longer follow up will provide further information.

\section{What is the Effect of Open vs. Percutaneous Screws on Complications Among Patients Undergoing Lateral Interbody Fusion for Adult Spinal Deformity?}

Khoi Duc Than, MD; Stacie Nguyen, BS, MPH; Paul Park, MD; Dean Chou, MD; Frank La Marca; Juan S. Uribe, MD; Todd D. Vogel, MD; Pierce D. Nunley, MD; Robert Eastlack, MD; Neel Anand, MD; Adam S. Kanter, MD; Praveen V. Mummaneni, MD; Gregory M. Mundis, MD; International Spine Study Group

Introduction: This study aims to compare differences in incidence and type of complications (COMP) between circumferential MIS (cMIS, i.e. MIS TLIF and/or LLIF with MIS screws) and hybrid approaches (HYB, i.e. MIS LLIF +/- with open screws).

Methods: A retrospective review of a multicenter MIS deformity database was performed. Inclusion criteria for this database: age $>18$ years, and at least one of the following: coronal Cobb angle $(\mathrm{CCA})>20, \mathrm{SVA}>5 \mathrm{~cm}, \mathrm{PI}-\mathrm{LL}>10$ and $\mathrm{PT}>20$. Only patients who underwent cMIS or HYB approaches were included for this study, and were propensity matched by levels instrumented.

Results: Of 420 patients that met inclusion criteria, 165 had complete data. 137 were available for analysis after excluding 3 column osteotomies and 76 remained after propensity matching (38 cMIS and 38 HYB). There were no differences in demographics, number of levels instrumented (6.8 HYB vs. $6.1 \mathrm{cMIS}$; $\mathrm{p}=0.622$ ), and pre- and post-operative radiographic results (Table 1). HYB had significantly longer OR time (623 v $490 \mathrm{~min}$; $\mathrm{p}=0.015)$ and larger EBL (1396 v 637; $\mathrm{p}=0.001) .55 .3 \%$ of HYB vs $44.7 \%$ cMIS patients suffered at least one COMP $(\mathrm{p}=0.359)$. cMIS patients had significantly fewer neurologic $(p=0.044)$, operative $(p=0.005)$, and minor $(p=0.034)$ COMPs. Reoperation was similar between groups (28.9\% HYB, 26.3\% cMIS). The most common COMP for cMIS was radiographic ( $\mathrm{n}=10 ; 6$ with pseudoarthrosis) and neurologic for HYB $(\mathrm{n}=11 ; 8$ with radiculopathy. Both groups saw improvement from pre to post op ODI, VAS back and leg (all $\mathrm{p}<0.05$ ), however cMIS had a greater reduction in VAS leg $(\mathrm{p}=0.002)$.

Conclusion: The overall COMP rate between cMIS and HYB is similar. CMIS surgery results in significantly fewer neurologic, operative, and minor COMP, Reoperation rates were similar, and despite complications patients saw significant improvement in pain and function.
147. Early Experience with a Hybrid Approach to Correcting Adult Degenerative Deformity: Combined MIS-LIF With SPO and Open Instrumentation

Oliver Flouty; Andrew James Grossbach, MD; Logan Helland, MD; Stephanus Viljoen, MD

Introduction: Minimally invasive (MIS) approaches to the thoracolumbar spine have recently been gaining popularity given their ability to reduce blood loss, operative time, and hospital stayl,3. Although these techniques have been successfully applied to the adult degenerative deformity patient, they have some limitations when addressing severe degenerative scoliosis3. Despite these shortcomings, the MIS lateral interbody fusion (LIF) offers several advantages1,2. Here we report our early experience with a hybrid approach utilizing MIS-LIF with open Smith-Peterson osteotomies (SPO) and posterior instrumentation to address adult degenerative deformity.

Methods: We reviewed the medical records of all patients who underwent a combined MIS-LIF and open multilevel SPO/ facetectomies and fusion spanning T10 to pelvis from January 2015 to June 2016. All patients underwent MIS-LIF across the apex of their curve. SPOs were performed at all levels that underwent interbody fusion. Included patients had at least one of the following: SVA $>6 \mathrm{~cm}$, LL-PI mismatch $>30$, or PT $>25.7$ patients were identified. 3 patients had preoperative and post-operative scoliosis films. Average preoperative and post-operative spinopelvic parameters were measured.

Results: Average postoperative measurements was pelvic incidence (PI) $49.5 \pm 3.2^{\circ}$, pelvic tilt (PT) $18.3 \pm 5^{\circ}$, Lumbar lordosis (LL) $34.3 \pm 13^{\circ}$, C7 sagittal vertical axis (SVA) $30 \pm 33.9 \mathrm{~mm}$ and coronal cob angle (CCA) $2.5 \pm 0.6^{\circ}$. Comparing pre- and postoperative scoliosis films showed an average reduction $(\mathrm{CI}=0.95)$ in $\mathrm{PI}$ by $18.3 \pm 12^{\circ}$, PT by $15.7 \pm 15^{\circ}$, and CCA by $16 \pm 11^{\circ}$. On the other hand, Lumbar lordosis showed an average improvement by $44.3 \pm 17^{\circ}$. Pre- and postoperative difference in C7-SVA measured $2 \pm 27 \mathrm{~mm}$. Average follow up was $12 \pm 8$ months. Complications included one patient with ureteral injury.

Conclusion: MIS-LIF combined with open posterior SPOs and instrumentation is a powerful tool for addressing adult degenerative deformity. Risks of combined approaches must be weighed against potential benefits including improved sagittal and coronal balance and improved fusion rates.

148. How Does Case Type, Length of Stay, and Comorbidities Affect Medicare DRG Reimbursement for Minimally Invasive Surgery (MIS) for Deformity?

Pierce D. Nunley, MD; Richard G. Fessler, MD, PhD; Paul Park, MD; Joseph M. Zavatsky, MD; Gregory M. Mundis, MD; Juan S. Uribe, MD; Robert Eastlack, MD; Dean Chou, MD; Michael Y. Wang, MD, FACS; Neel Anand, MD; Adam S. Kanter, MD; Stacie Nguyen, BS, MPH; Christopher I. Shaffrey, MD, FACS; Praveen V. Mummaneni, MD; International Spine Study Group

Introduction: We investigated Medicare DRG based reimbursement for MIS deformity procedures in our study group hospitals based on length of stay and presence of comorbid conditions (CC).

Methods: DRG based reimbursement was obtained for MIS anterior, posterior and circumferential 1-level and multi-level fusion for listhesis and deformity cases with and without CC from 12 institutions throughout the US. The 3 most common MIS procedures were analyzed to compare reimbursement based on DRG coding: 1 . Fusion via anterior or posterior only; 2. Fusion anterior with fixation posterior percutaneous (no dorsal fusion); 3. Fusion Combined anterior and posterior.

Results: The number of levels fused does not affect the 
reimbursement for all cases. Cases 1 and 2 without $\mathrm{CC}, 3$-day stay reimbursed $\$ 41,404$ vs 8 -day reimbursed $\$ 42,808$. Cases 1 and 2 with CCs, 3-day stay reimbursed $\$ 54,476$ vs 8 -day stay reimbursed $\$ 55,881$. Case 3 without CC, 3 -day stay reimbursed $\$ 47,992$ vs 8 -day stay reimbursed $\$ 49,397$. Case 3 with CC, 3-day reimbursed $\$ 61,806$ vs 8 -day reimbursed $\$ 63,212$. The increased payment for an 8 -day stay was $\$ 1,405$ or $\$ 281$ per day. If a deformity case 1 or 2 is coded incorrectly as a degenerative case the decrease in payment was $\$ 9,769$ lower (-24\%) with no CC and \$22,841 lower (-42\%) with CC.

Conclusion: Regardless the direct costs, Medicare DRG based reimbursement was the same for single and multi-level MIS deformity cases. The use of posterior percutaneous fixation without dorsal fusion resulted in a $13-16 \%$ lower reimbursement compared with the addition of a posterior arthrodesis. Coding a deformity case as degenerative by the hospital resulted in $24-42 \%$ lower DRG based reimbursement. In today's challenging environment it is important that physicians and hospitals better understand procedure and coding issues in order to be able to continue to offer complex spinal surgeries cost effectively to our patients.

\section{Current MIS Techniques Fail to Optimize Spinopelvic} Parameters in Patients With High Pelvic Incidence

Gregory M. Mundis, MD; Robert Eastlack, MD; Praveen V. Mummaneni, MD; David O. Okonkwo, MD, PhD; Stacie Nguyen, BS, MPH; Adam S. Kanter, MD; Neel Anand, MD; Paul Park, MD; Pierce D. Nunley, MD; Juan S. Uribe, MD; Joseph M. Zavatsky, MD; Dean Chou, MD; Vedat Deviren, MD; International Spine Study Group

Introduction: High pelvic incidence (HPI) demands a larger lumbar lordosis (LL) to achieve ideal spinopelvic harmony. Criticisms of MIS spine surgery (cMIS: MIS ant/lateral with MIS screws) include challenges in adequately matching LL to PI. This study analyzes the radiographic and clinical outcomes of patients treated with MIS with varying PIs.

Methods: Retrospective review of multicenter MIS database was queried for cMIS patients. Patients were grouped as low (LPI=43; $n=14)$, mid $(41<\mathrm{MPI}<66 ; \mathrm{n}=46)$, and high $(\mathrm{HPI}=67 ; \mathrm{n}=17))$ pelvic incidence, by using 1 standard deviation from the mean. Theoretical LL (tLL) was calculated based on Schwab PI-LL formula with respect to PI outliers: $\mathrm{tLL}=\mathrm{LPI}+10,=\mathrm{MPI},=\mathrm{HPI}-10$. The offset was calculated as the difference between tLL and pre- or post-LL. Well aligned (WA) patients were classified to Vialle et al. Nonparametric Kruskal-Wallis test was used to assess significant differences between groups.

Results: 420 patients were available for review, 165 patients identified in the database and 77 met inclusion. There were no differences for demographics, levels treated, iliac fixation or use of lateral interbody. At baseline LPI and MPI had lower PT than HPI $(15.7$ v 23.5 v $33.6 ; p<0.05)$ and preop PI-LL lower for LPI than HPI ( 9 v 21.9; $<<0.05$ ) with no difference in SVA or max Cobb. Post op both LPI and MPI had improved LL, but HPI did not. The tLL, and offset however, was not different between groups at pre or postop ( $>0.05$ ). All groups saw improvement in ODI, VAS back and leg, with no difference in reaching MCID. Complication occurrence was similar between groups.

Conclusion: Patients with HPI remain some of the most difficult spinal deformities to treat. Current MIS techniques for treating this patient population reveal the inability to correct the PI-LL mismatch, likely due to the need for more lordosis. Consideration should be given when employing MIS techniques in patients with a high PI.
150. Early and Late Reoperation Rates and Etiologies are Similar Between cMIS and HYB Techniques For ASD Correction

Robert Eastlack, MD; Gregory M. Mundis, MD; Stacie Nguyen, BS, MPH; Praveen V. Mummaneni, MD; David O. Okonkwo, MD, PhD; Adam S. Kanter, MD; Neel Anand, MD; Paul Park, MD; Pierce D. Nunley, MD; Juan S. Uribe, MD; Joseph M. Zavatsky, MD; Dean Chou, MD; Vedat Deviren, MD; International Spine Study Group

Introduction: Reoperation after correction of adult spinal deformity (ADS) results in additional cost and morbidity. The rates and etiologies of reoperations may be impacted by the method of ASD correction. We aimed to characterize the reoperation rates and etiologies when performing ASD surgery with cMIS and HYB techniques.

Methods: A multicenter database was queried. Inclusion criteria for the database included age $=18$ years, and one of the following: $\mathrm{CC}>20, \mathrm{SVA}>5 \mathrm{~cm}, \mathrm{PT}>20, \mathrm{PI}-\mathrm{LL}>10$. Patients with either circumferential MIS (cMIS) or hybrid (HYB) surgery, and $=3$ spinal levels treated with 2-year minimum follow-up were included for analysis.

Results: 420 patients met inclusion criteria for the database. Of those 165 had complete 2-year data, and a total of 133 met inclusion for this study (65 HYB and 68 cMIS). Junctional failure (15.4\%) was the most common reason for reoperation in the HYB group, while fixation failure was the most common reason in the cMIS group (8.8\%) (Table 1). There was a higher incidence of PJF than DJF within HYB $(12.3 \%$ vs. $3.1 \%)$, but no significant differences in PJF or DJF rates when compared to cMIS. There were no other differences between cMIS and HYB when analyzing reasons for reoperation. Early ( $<30$ days) reoperations were less common in both groups $(\mathrm{cMIS}=1.5 \% ; \mathrm{HYB}=6.1 \%)$ than late $(>30$ days) reoperations (cMIS $=26.5 \%$; HYB $=27.7 \%$ ), but rates were similar between groups.

Conclusion: ASD correction with CMIS and HYB techniques result in overall reoperation rates of $27.9 \%$ and $33.8 \%$, respectively, at minimum 2-year follow-up. Junctional failures are more common after HYB approaches, while pseudarthrosis/fixation failures happen more often with cMIS techniques. Early reoperations were less common than later returns to the OR in both groups. These reoperation rates compare favorably with open ASD surgery, although the reasons for failure may differ. Further study will be done to evaluate the specific differences between reoperation etiologies when comparing open vs. MIS ASD correction.

151. Effect of Intraoperative Epidural Methylprednisolone in Patients Undergoing MIS Single Level Microdiscectomy, A Prospective Randomized Control Study of 220 Patients

Tianyi Niu, MD; Derek Stephen Lu, MD; James Leiter, MD; Daniel C. $L u, M D$

Introduction: Microdiscectomy is an effective treatment for radiculopathy secondary to disc herniation. Intraoperative epidural steroid has been used by some authors after the decompression; however, this practice is not universally adopted. A meta-analysis in 2015 showed that intraoperative epidural steroid use may carry a small increased risk of infection with mildly improved short-term pain relief. We present our own large cohort, prospective, randomized control trial

Methods: A single center, multi-surgeon, randomized control trial of 220 individuals undergoing minimally invasive tubular surgery, single level lumbar microdiscectomy. 108 individuals received 40 $\mathrm{mg}$ methylprednisolone intraoperatively to the effected nerve root after the discectomy, while the other 112 individuals only received microdiscectomy. For each subject, visual analog scale (VAS) back and leg pain, Oswestry Disability Index (ODI), Short From-12 (SF-12) and narcotic pain medication consumption were recorded at preop, 
1-day post-op, 2-weeks post-op, 3-months follow-up, and 12-months follow-up.

Results: There were no differences in patient profiles between the two groups (age, gender, BMI, co-morbidities, pain medication use, and chronicity of symptoms). Furthermore, there was no difference in the pre-operative metrics of VAS, ODI or SF-12. After surgery, VAS (2.0 vs. 2.4 , steroids vs. no steroids, $\mathrm{p}=0.06)$ and ODI (18 vs. 22 , steroids vs. no steroids, $\mathrm{p}=0.057$ ) tended to be better in the methylprednisolone group, which persisted to 3-months follow-up. At 12-month follow-up, such trends were not obvious. There were no adverse events associated with intraoperative methylprednisolone use. There were no wound infections or hematoma formation. There were 2 cases of recurrent disc herniation in the steroid treatment cohort (treated conservatively) and 1 case in the non-steroids cohort (that was treated with surgery).

Conclusion: Intraoperative methylprednisolone appears to modestly improve pain and disability of patients undergoing minimally invasive tubular microdiscectomy in the short-term (less than 3 months), though the improvement is not statistically significant. Methylprednisolone use was not correlated with significant sideeffects. Based on our study, there is no evidence to support the use of intraoperative methylprednisolone in single level MIS lumbar microdiscectomy.

152. Microsurgical Foraminotomy Via Wiltse Paraspinal Approach for Foraminal or Extraforaminal Stenosis at L5-S1 Level: Risk Factor Analysis for Poor Outcome

Won Mo Gu; Sung Ik Cho

Introduction: There have been only a few studies on preoperative risk factors for persistent or recurrent radiculopathy. We investigated risk factors associated with poor outcome of microsurgical foraminotomy at L5-S1 level.

Methods: We analyzed 21 patients who underwent the microsurgical foraminotomy for FEF stenosis at L5-S1 level. To investigate risk factors associated with poor outcome, patients were classified into two groups (success and failure in foraminotomy). Clinical outcomes were assessed by the visual analogue scale (VAS) scores of back and leg pain and Oswestry disability index (ODI). Radiographic parameters including existence of spondylolisthesisa, existence and ${ }^{\circ}$ of coronal wedging, disc height, foramen height, segmental lordotic angle (SLA) on neutral and dynamic view, segmental range of motion, and global lumbar lordotic angle were investigated.

Results: Postoperative VAS score and ODI improved after foraminotomy. However, there were 7 patients (33\%) who had persistent or recurrent leg pain. SLA on neutral and extension radiographic films were significantly associated with the failure in foraminotomy $(p<0.05)$. Receiver-operating characteristics curve analysis revealed the optimal cut-off values of SLA on neutral and extension radiographic films for predicting failure in foraminotomy were 17.3 and $24^{\circ} \mathrm{s}$, respectively.

Conclusion: Microsurgical foraminotomy for FEF stenosis at L5-S1 level can provide good clinical outcomes in selected patients. Poor outcomes were associated with large SLA on preoperative neutral $\left(>17.3^{\circ}\right)$ and extension radiographic films $\left(>24^{\circ}\right)$.
153. Percutaneous Pedicle Screw Fixation After Direct Spinal Canal Decompression in Magerl Type A3 Thoracolumbar Burst Fractures with Neurological Injury: An Alternative Option

Jung-Kil Lee, MD; Bong Ju Moon; Ki-Young Choi

Introduction: Utilization of a posterior approach to the spine for placement of pedicle screws for fixation has become a popular treatment of TLBFs. PPSF systems have increasingly been used, but not explored in combination with decompression for TLBFs with neurological injury. To investigate the surgical results of percutaneous pedicle screw fixation (PPSF) after spinal canal decompression via a small laminotomy for the treatment of thoracolumbar burst fractures (TLBFs) with neurological deficits

Methods: Seventeen patients who had neurological injury after a thoracolumbar burst fracture and underwent decompression followed by immediate percutaneous pedicle screw fixation were retrospectively reviewed for operative time, length of hospital stay, Cobb angle, vertebral wedge angle, and vertebral body index. A sample of 17 patients sustaining neurological injury due to a single-level thoracolumbar burst fracture. Operative time, length of hospital stay, neurological assessment as determined using the Frankel grading system, Cobb angle, vertebral wedge angle, and vertebral body index were measured. Seventeen patients underwent PPSF after spinal canal decompression via small laminectomies between April 2009 and April 2015. Inclusion criteria consisted of a single-level TLBF and neurological deficits. Patients with multiple or pathological fractures, or polytrauma were excluded. Decompression was performed via a small laminectomy, followed by PPSF, including at the level of the fractured vertebra.

Results: The average follow-up period was 20 months (range 6 to 62 months). The average Cobb angle was $15.77^{\circ} \pm 6.64^{\circ}$, and significantly decreased to $6.54^{\circ} \pm 6.22^{\circ}$ postoperatively $(\mathrm{p}<0.001)$. The average vertebral wedge angle was $20.58^{\circ} \pm 6.26^{\circ}$, and decreased significantly to $12.21^{\circ} \pm 6.22^{\circ}$ postoperatively $(\mathrm{p}<0.001)$. The vertebral body index significantly decreased from $0.58 \pm 0.11$ to a postoperative value of $0.78 \pm 0.10(p<0.001)$. No patient deteriorated subsequent to surgery

Conclusion: Percutaneous pedicle screw fixation after spinal canal decompression via small laminotomy provides significant kyphotic correction and improved neurological outcome while offering decreased surgical morbidity. This may be applied as an effective primary surgery in select patients with TLBFs with neurologic deficits

155. MRI Analysis of the Combined AOSpine North America and International Studies: The Prevalence and Spectrum of Pathologies in a Global Cohort of Patients With Degenerative Cervical Myelopathy

Aria Nouri, MD, MSc; Allan R. Martin, BASc, MD; Lindsay Tetreault, Bsc; Anick Nater, MD, BS; So Kato; Hiroaki Nakashima, MD; Narihito Nagoshi, MD, PhD; Hamed Reihani-Kermani, MD; Michael G. Fehlings, MD, PhD, FRCS(C), FACS

Introduction: Degenerative Cervical Myelopathy (DCM) encompasses a spectrum of conditions, including spondylosis and ossification of the posterior longitudinal ligament (OPLL), which result in progressive spinal cord impairment. Through detailed review of MRIs, the global prevalence of degenerative pathologies of patients with DCM is reported.

Methods: MRIs of 458 patients (age 56.4 +/-11.8, 285 Male, 173 Female) were obtained from North America $(n=200)$, Europe $(n=93)$, Latin America $(n=58)$ and Asia-Pacific $(n=107)$ and assessed for the type of pathology, directionality of stenosis, level of maximum cord compression, levels of spinal cord compression, and signal changes on T2WI and T1WI. Data were analyzed for differences between 
sex using Chi-square tests and geographic variations using KruskalWallis tests.

Results: Globally, spondylosis was most frequently present $(89.7 \%)$ and was commonly accompanied by enlargement of the ligamentum flavum (LF) (59.9\%). Single level disc pathology, OPLL and spondylolisthesis had a prevalence of $\sim 10 \%$ each. OPLL was accompanied by spondylosis in $91.7 \%$. Associated abnormalities such as Klippel-Feil Syndrome and congenital stenosis were observed in $2.0 \%$ and $8.4 \%$, respectively. The Asia-Pacific region had more OPLL $(29 \%, \mathrm{p}=3 \mathrm{x} 10-11)$ and less spondylolisthesis $(1.9 \%, \mathrm{p}=0.002)$. Females presented more commonly with single level disc pathology $(13.9 \%$ vs. $6.7 \%$; $\mathrm{p}=0.013)$, and males with spondylosis $(92.3 \%$ vs. $85.6 \% ; p=0.02)$ and enlargement of LF (61.4\% vs. $49.1 \% ; p=0.01)$. C5-6 was the most frequent maximum compressed site (39.5\%) and region for T2WI hyperintensity $(38.9 \%)$. T2WI hyperintensity more commonly presented in males ( $82.4 \%$ vs. $66.7 \%$; $p<0.001)$.

Conclusion: This is the largest report on the prevalence and spectrum of pathology in patients with DCM. Herein it has been demonstrated that degenerative features are highly interrelated, that females presented with milder MRI evidence of DCM, and that variations exist in the prevalence of pathologies between geographical regions.

\section{Assessment of Gait Disturbance by Foot Pressure Measurement in Patients with Myelopathy}

Tetsuryu Mitsuyama, MD, PhD; Yoshikazu Okada; Takakazu Kawamata

Introduction: Gait disturbance by myelopathy is mainly assessed with subjective complains of patients, whereas myelopathy hand is objectively assessed with the frequency of finger grip and release in ten minutes and grading of finger escape sign. Foot pressure measurement is one of the methods for gait analysis and some measuring instruments are commercialized as simplified mobile systems. Unstable on tandem gait and Romberg sign reflect motor and/or sensory ataxia in patients with myelopathy. The purpose of this study is to verify the quantitative assessments of tandem gait tests and Romberg tests with foot pressure measurements for patients with gait disturbance by myelopathy.

Methods: We measured the foot pressure distribution in 31 patients with myelopathy, who underwent spinal surgery. The tests consisted of normal walking in 20 meters, tandem gait in 5 meters (Figure 1), standing with eyes opened closed in 15 seconds (Figure 2). We scored the center line of foot pressure for each test on 4-points scale. Motor function of the lower extremity was assessed with the modified JOA Score. They were evaluated before and after surgery.

Results: Preoperative and postoperative scores of motor function were $1.9 \pm 0.73$ and $3.1 \pm 0.98$. Scores of tandem gait test were $1.6 \pm 0.98$ and $2.3 \pm 1.2$, respectively. Scores of Romberg test were $2.0 \pm 0.71$ and $2.7 \pm 0.91$, respectively. We evaluated tandem gait test and Romberg test about six months after surgery in 25 patients. Scores tandem gait additionally recovered from $2.4 \pm 1.1$ to $3.1 \pm 0.83$. Scores of Romberg test was also recovered from $2.8 \pm 0.93$ to $3.2 \pm 0.72$.

Conclusion: Foot pressure measurements graphically showed that tandem gait tests and Romberg tests reflect the postoperative recovery of gait disturbance in patients with myelopathy. They could be useful not only in postoperative evaluation, but also in determining surgical patients with benign spinal tumor or degenerative cervical stenosis.
157. A Clinical Practice Guideline for the Management of Patients with Degenerative Cervical Myelopathy: Recommendations for Patients with Mild, Moderate and Severe Disease and Nonmyelopathic Patients with Evidence of Cord Compression

Lindsay Tetreault, Bsc; Bizhan Aarabi, MD; Paul M. Arnold, MD; Darrel S. Brodke, MD; Anthony Burns, MD; Simon Carette; Robert Chen, MA, MBBChir, MSc, FRCPC; Kazuhiro Chiba; Joseph Dettori; Julio C. Furlan, MD, PhD, MBA, MSc; James S. Harrop MD, FACS; Langston T. Holly MD; Sukhvinder Kalsi-Ryan, BScPT, MSc, PhD; Mark Kotter; Brian K. Kwon, MD, PhD, FRCSC; Allan R. Martin, BASc, MD; James Middleton; James Milligan; Hiroaki Nakashima, MD; Narihito Nagoshi, MD, PhD; John Rhee, MD; K. Daniel Riew, MD; Anoushka Singh; Andrea Skelly, PhD; Sumeet Sodhi; Jeffrey C. Wang; Jefferson R. Wilson MD, PhD; Albert Yee, MD; Michael G. Fehlings, $M D, P h D, F R C S(C)$, FACS

Introduction: This study aims to develop guidelines that outline how to best manage (1) patients with mild, moderate and severe myelopathy and (2) nonmyelopathic patients with evidence of cord compression with or without clinical symptoms of radiculopathy.

Methods: Five systematic reviews were conducted to synthesize evidence on disease natural history; risk factors of disease progression; the efficacy, effectiveness and safety of nonoperative and surgical management; the impact of preoperative duration of symptoms and myelopathy severity on outcomes; and the frequency, timing and predictors of symptom development. A multidisciplinary group used this information, in combination with their clinical expertise, to develop recommendations for the management of DCM.

Results: Our recommendations were: (1) "We recommend surgical intervention for patients with moderate and severe DCM;" (2) "We suggest offering surgical intervention or a supervised trial of structured rehabilitation for patients with mild DCM. If initial non-operative management is pursued, we recommend operative intervention if there is neurological deterioration and suggest operative intervention if the patient fails to improve;" (3) "We suggest not offering prophylactic surgery for non-myelopathic patients with evidence of cervical cord compression without signs or symptoms of radiculopathy. We suggest that these patients be counseled as to potential risks of progression, educated about relevant signs and symptoms of myelopathy, and be followed clinically;" and (4) "Non-myelopathic patients with cord compression and clinical evidence of radiculopathy with or without electrophysiological confirmation are at a higher risk of developing myelopathy and should be counselled about this risk. We suggest offering either surgical intervention or non-operative treatment consisting of close serial follow-up or a supervised trial of structured rehabilitation. In the event of myelopathic development, the patient should be managed according to the recommendations above."

Conclusion: These guidelines should be implemented into clinical practice to improve outcomes and reduce morbidity in patients with DCM.

158. National Trends in Demographics and Outcomes Following Cervical Fusion for Cervical Spondylotic Myelopathy

Caroline E. Vonck, BS; Joseph E. Tanenbaum, BA; Gabriel Alexander Smith, MD; Edward C. Benzel, MD; Thomas Mroz; Michael P. Steinmetz, MD

Introduction: Cervical fusion is a common surgical modality used in the treatment of cervical spondylotic myelopathy (CSM). It is unclear how national trends in the surgical management of CSM have evolved since 2002.

Methods: The National Inpatient Sample (NIS) was used to identify adult patients with CSM (International Classification of Disease, Ninth Revision, Clinical Modification [ICD-9-CM] code 721.1) who underwent cervical fusion from 2003-2013. Patients were 
stratified based on procedure: anterior cervical fusion (ACF) or posterior cervical fusion (PCF) (ICD-9-CM codes 81.02 and 81.03, respectively). Data points included age, gender, race, comorbidity burden, primary insurance beneficiary, hospital size, geographic location, teaching status, and the outcome measures of in-hospital mortality, length of stay (LOS), and hospital charges. Chi-square tests were performed to compare categorical variables. Independent t-tests using the Satterthwaite method were performed to compare continuous variables.

Results: We identified 62,970 patients with CSM who underwent cervical fusion from 2003-2013. The number of fusions performed per year to treat CSM increased from 3,879 to 8,181. Patients tended to be male, white, and insured privately (ACF) or by Medicare (PCF). The average age of fusion patients increased from 58.2 to 60.6 years $(p<0.001)$. The percentage of patients with three or more comorbidities increased from $14.8 \%$ to $34.3 \%(p<0.001)$. Fusions were increasingly performed at smaller hospital centers. LOS did not change significantly from a mean of 3.7 days. In-hospital mortality decreased from $0.6 \%$ to $0.3 \%(p<0.01)$. Hospital charges increased from $\$ 49,445$ to $\$ 92,040$ ( $\mathrm{p}<0.001)$. Trends for ACF and PCF were broadly similar to the all fusions cohort.

Conclusion: This study showed a dramatic increase in cervical fusions to treat CSM from 2003-2013. Despite increases in average age and number of comorbidities, smaller hospitals performed more fusions for CSM with LOS remaining constant and mortality decreasing across the study period. However, hospital charges increased dramatically.

159. Comparative Effectiveness Between Laminectomy with Fusion and Laminoplasty for the Treatment of Multilevel Cervical Spondylotic Myelopathy

Heath P. Gould, BS; Colin Haines; Emily Hu, BA; Jacob A. Miller, BS; Roy Xiao, BA; Thomas E. Mroz, MD; Don Moore, MD

Introduction: The optimal management of multilevel cervical spondylotic myelopathy (CSM) remains unknown. Given the cost of spinal fusion, laminoplasty (LP) may represent a more cost-effective alternative to laminectomy with fusion (LWF) that is equally efficacious.

Methods: A retrospective cohort study was conducted among patients undergoing cervical decompression for the treatment of multilevel CSM. The EQ-5D (quality of life), PDQ (pain-related disability), and PHQ-9 (depression) instruments were prospectively-collected between 2008 and 2015. The surgical episode of care was defined in three periods: 30 days prior to admission, the index admission, and discharge to 365 days after admission. Surgical costs were normalized to national Medicare reimbursement and presented in 2014 USD.

Results: 186 patients were eligible for inclusion; among these, 142 (76\%) underwent LWF, while 44 (24\%) underwent LP. Preoperatively, the mean EQ-5D index was marginally greater in the LP cohort ( 0.530 vs. $0.581, p=0.17)$, demonstrating poorer preoperative quality of life (QOL) in the LWF cohort. Postoperatively, mean EQ-5D index improved to $0.592(\mathrm{p}=0.02)$ and $0.664(\mathrm{p}=0.01)$ in the LWF and LP cohorts, respectively. Total episode of care costs were greater in the LWF cohort ( $\$ 34,718$ vs. $\$ 25,260, p<0.001)$. Following multivariable linear regression, total episode of care costs remained significantly greater in the LWF cohort $(\beta=0.572, \mathrm{p}<0.001)$, corresponding to a mean difference of $\$ 16,392$.

Conclusion: Poorer preoperative QOL was observed among patients undergoing LWF relative to LP, and therefore these populations may differ with respect to expected surgical benefit. However, the proportion of patients achieving a clinically-relevant QOL improvement did not significantly differ, suggesting similar efficacy. After controlling for differences in baseline characteristics, LP appeared to be more cost-effective relative to LWF.
160. In Continous Type Ossification of Posterior Longitudinal Ligament, Which One is Treatment of Choice: Laminectomy or Laminoplasty?

Yong-Eun Cho; Sun-Joon YOO; Dal-Sung Ryu; Kyung-Hyun Kim; Jeong-Yoon Park; Sung-Uk Kuh; Dong-Kyu Chin; Keun-Su Kim

Introduction: The fundamental principle underlying laminoplasty is optimal preservation of the posterior supporting elements in the cervical spine and prevention of weakening of the structural support, while in laminectomy, posterior tension band is easily get lost or damaged. The ossification foci is believed to create an anterior column support as a stabilizer and offer biomechanical prevention of postoperative kyphotic change after multilevel laminectomy. This study was conducted to assess comparatively surgical outcome of laminectomy versus laminoplasty.

Methods: We reviewed medical data in 646 patients who underwent cervical OPLL surgery from 2004 to 2014 at our institution. Inclusion criteria were diagnosis of Continuous type of OPLL, minimal follow up more than 24 months. Among them, 45 laminectomy patients and 45 laminoplasty patients were enrolled. C27 SVA, Cobb angle and T1 slope were measured at each patient.

Results: The mean age of patient was 64 and 61 years at each group, and the mean follow up period was laminectomy group 41 months and laminoplasty group 35 months. Basic characteristics including age, sex, mean f/u were not different each groups. Clinical outcome including NDI, JOA, VAS scores showed improvement in both groups $(p<0.0001)$. C27 SVA was not different in each group at preoperative status and steadily increased overtime ( $p<0.0001)$. However, SVA of laminectomy group became lager than that of laminoplasty group at post op 1 year and last follow up. C27 cobb angle significantly decreased over time $(p<0.0001)$, however there was no difference between groups. Incidence of kyphotic alignment which means $\mathrm{C} 27$ cobb angle less than negative 5 degree was evaluated. This result shows that incidence of kyphotic alignment in laminectomy group was higher than laminoplasty group. Each group ended up showing kyphotic alignment in 11 laminectomy patients and 2 laminoplasty patients at last follow up $(p=0.014)$. T1 slope was constant factor regardless of time and operation $(\mathrm{p}=0.986)$.

Conclusion: Both posterior decompression surgery showed significant clinical improvement. But Loss of lordosis proceeded each groups as time goes on. Laminectomy increased the incidence of kyphotic alignment in continuous OPLL, this means ossification foci was not enough to support spinal column. The posterior tension band is neccessory to stablize the cervical column and prevent kyphotic change Finally we conclude that laminoplasty is more effective treatment in continuous OPLL as in other type of OPLL.

162. Correlation Between Cervical Sagittal Alignment Changes and T1 Slope of the Patients with Cervical Spondylotic Myelopathy and Cervical Ossification of the Posterior Longitudinal Ligament After Laminoplasty

Kang Ji In; Farid Yudoyono; Dong Ah Shin; Byeong Woo Kim; Zhimin Pan; Keung Nyun Kim; Do Heum Yoon; Seong Yi; Yoon Ha

Introduction: Laminoplasty is a surgical method to treat patients with cervical ossification of the posterior longitudinal ligament(COPLL) and cervical spondylothic myelopathy (CSM). Previous study reported, patients with COPLL and CSM demonstrate postoperative alignment change. Recently, cervical T1 slope has background as a predictor of alignment change after laminoplasty. The study on the effects of T1 slope on cervical sagittal alignment change between COPLL and CSM is not yet fully established.

Methods: Between January 2009 and January 2012, 56 consecutive patients who underwent cervical laminoplasty for 
COPLL were enrolled (male:female ratio $=48: 8$; mean age $=53.9$ years) and 51 consecutive patients who underwent cervical laminoplasty for CSM were enrolled(male:female ratio $=39: 12$; mean age $=60.7$ years). Cervical spine lateral radiographs in neutral, flexion, and extension were taken before surgery and at final follow-up. The C2-C7 Cobb angle, C2-C7 cervical range of motion (ROM), T1 slope (T1S), and C2-C7 sagittal vertical axis (SVA), loss of cervical lordosis (LCL) were measured from lateral radiographs of the cervical spine preoperatively and postoperatively at final follow-up. The authors retrospectively compared according to preoperative $\mathrm{T} 1$ slope patients with a diagnosis of cervical stenosis due to either COPLL or CSM who had been treated with laminoplasty. Postoperative alignment and LCL incidence were also evaluated at final follow-up. The correlation between postoperative cervical sagittal alignment change and preoperative variables, including age, T1 slope, C2-C7 ROM, C2-C7 SVA were investigated.

Results: Patients were divided into two groups above and below mean preoperative $\mathrm{T} 1$ slope $\left(26.6^{\circ}\right)$. There were significant differences in sex in COPLL group and in CSM group, male was predominance in both group $(p=0.008 ; p=0.01$ ). Patients in CSM group with high T1 slope demonstrated significantly more lordotic cervical alignment preoperatively $\left(17 \pm 9.5^{\circ} ; \mathrm{p}=0.01\right)$. Patients in CSM group with high T1 slope were likely tended to more sagittal alignment changes postoperatively than COPLL group $\left(4.33^{\circ} \pm 7.08^{\circ} ; 7.49^{\circ} \pm 4.87^{\circ} ; \mathrm{p}=0.7\right)$. In the CSM group the changes in alignment significantly correlate with preoperative sagittal vertical axis $(r=0.4 ; p=0.02)$. Patients in COPLL group with high T1 slope were higher sagittal vertical axis change than CSM postoperatively $(24.12 \pm 25.43 ; 19.07 \pm 25 ; p=0.4)$.

Conclusion: CSM Patients group with higher T1 slope had more lordotic curvature before surgery. Among patients who have undergone laminoplasty, those with CSM more cervical sagittal alignment changes than those with COPLL group for sagital vertical axis changes COPLL group more than CSM group at final follow-up.

163. Mid-term Follow Up of Subsidence Rate in Flanged Mesh Cage Reconstruction Without Plating in Anterior CSM/OPLL Surgery

\section{Soo-Bin Im}

Introduction: Anterior cervical corpectomy is a well-established surgical procedure that provides the direct decompression and sufficient space to remove pathology causing cord compression, such as an cervical spondylotic myelopathy(CSM) and ossified posterior longitudinal ligament (OPLL). Augmentation by anterior plating is routine, but it needs wider surgical exposure, cost and time. However, anterior plating is still doubtful that it has real benefit for stabilization of corpectomy reconstruction. we introduced flanged mesh cage reconstruction (FMCR) instead of using anterior plate and investigated its the subsidence rate and radiological outcome.

Methods: Between 2012 and 2014, retrospective review of the radiological and clinical data of 44 patients with CSM and/or OPLL who were operated FMCR without anterior plating. The tailored mesh cage have two small flanges in the upper and lower -anterior corner and the flange was positioned to the cortical rim adjacent vertebral bodies. The cancelous screws were inserted through the hole of flange in some cases. The subsidence rate was defined as the change in the average height of the involved segments between initial and follow-up x-ray and measured at 3, 6, 12 months. Cases presenting subsidence more than $3 \mathrm{~mm}$ were classified as the noticeable subsidence.

Results: OPLL and CSM was each was 22. The mean subsidence rate was $2.1 \mathrm{~mm}$ and developed mainly within 3 months. The noticeable subsidence was developed in 10 cases $(22 \%)$. Screw breakage was found in one case, but there was no dislodgement of cage or screw pull-out and fusion was achieved in all cases. There was no statistical relationship between the subsidence rate and extent of corpectomy ( $\mathrm{p}=0.598)$.

Conclusion: The FMCR without anterior plating for CSM/ OPLL resulted in favorable fusion and acceptable subsidence rates comparing to reconstruction with plate in the literature. Subsidence rate was not related with extent of corpectomy.

\section{CrossFit Related Spinal Injuries: A Retrospective Study}

Benjamin Hopkins, BS; Michael Brendan Cloney, BA; Kartik Kesavabhotla; Adeola L. Adeoye, APN; Zachary A. Smith, MD; Tyler R. Koski, MD; Wellington Hsu, MD; Nader S. Dahdaleh, MD

Introduction: CrossFit is an explosively popular form of high intensity exercise involving heavy weight-lifting, gymnastics, pullups, and rope climbing combined into short, repetitive, high intensity workouts. Case reports have documented injuries ranging from muscle tears to severe exercise induced rhabdomyolysis and surveys suggest that as many as $7 \%$ of documented CrossFit injuries go on to require surgical intervention. The purpose of this study is to examine our experience with spine related injuries associated with high intensity CrossFit workouts

Methods: All patients who presented to Northwestern Memorial Hospital and its affiliated clinics complaining of an injury sustained performing CrossFit between June 2010 and June 2016 were identified using the Northwestern University Electronic Data Warehouse (EDW). Patients who experienced back or neck pain underwent a full chart review. The data collected included the age, sex, BMI, CrossFit experience level, symptom duration, type of symptoms, type of clinic presentation, cause of injury, objective neurological exam findings, imaging type, number of clinic visits and treatments prescribed.

Results: 95 patients were identified. The mean age was 37.1 years. The majority of the patients were male $(55.7 \%)$. The average BMI was 26.8. The most common location of injury was the lumbar spine with $79(83.2 \%)$ of patients. The average symptom duration was 6.5 months. Radiculopathy was reported in $52.6 \%$ of cases. About $50 \%$ of patients received spinal imaging. The most commonly prescribed treatment was physical therapy; 37 (38.9\%) patients. Surgery was recommended for $10(10.5 \%)$ of patients presenting. $7(7.4 \%)$ of the initial 95 patients underwent surgical intervention after failing conservative treatment. The most commonly performed procedure was a lumbar discectomy.

Conclusion: CrossFit is a popular, high intensity style workout with the potential of causing spinal injuries. We observe a 7\% rate of surgical intervention in patients who present to medical attention with spine related injury.

165. Isolated Occipital Condyle Fractures are Unlikely to Result in Delayed Instability Regardless of Management Style

Nathan Davis; Nathan Quig, BS; Casey Hribar; Deb A. Bhowmick, $M D$

Introduction: Occipital condyle fractures (OCF) are being detected at a higher frequency with standardized cross-sectional imaging for trauma evaluation. While previous studies suggest OCF are largely stable in nature, no evidence exists guiding standard management. We hypothesized that all isolated OCFs can be managed conservatively without long-term consequences.

Methods: A retrospective chart review was undertaken at our institution for OCF from 2005-2015 with at least 6 months of clinical and radiographic follow-up. Failure of treatment was defined as early or late instability defined by radiographic or clinical exam changes that may be attributed to cranial settling or neural axis compression.

Results: A total of 54 patients met the inclusion criteria. The average age was 34 years old with a range of 11 years old to 86 years old. One patient had bilateral OCF and 53 had unilateral OCF. 28 
patients had no additional spine fractures, 13 had cervical spine fractures, 2 thoracic spine fractures, 4 with lumbar spine fractures and 7 had fractures in multiple regions. At the time of discharge, 39 OCF were managed with rigid cervical collar, 10 with no orthosis, 2 with a Minerva brace, and 1 with a halo. Two patients required cervical surgery; an occipital cervical fusion for a type 2 odontoid fracture and one atlantoaxial fusion for atlantoaxial instability was performed. At the follow up appointments, none of the patients were found to have delayed instability based on clinical examination, upright $\mathrm{x}$-rays or flexion-extension x-rays.

Conclusion: All isolated OCF are likely stable injuries. Our data suggests all isolated OCF may be treated conservatively with any type of cervical orthosis and minimal follow up. Given our small cohort of patients treated without orthosis, it is possible that isolated OCF may be treated without cervical orthosis at all.

\section{A Finite Element Analysis of the Occitipoatlantal Capsular Ligaments as the Primary Stabilizers of the Craniocervical Junction}

Andrew T. Dailey, MD; Rinchen Phuntsok; Douglas L. Brockmeyer, MD; Benjamin Ellis

Introduction: Cadaveric biomechanical testing of the craniocervical junction (CCJ) demonstrates that the occipitoatlantal (OA) joint stability relies on three ligamentous structures: tectorial membrane (TM), transverse ligament (TL) and occipitoatlantal capsular ligaments (OACL). Previously, the TM is thought to play the dominant role in stability. Stability studies involving transection of OACL are less common. In this study, our goal is to examine the contributions of the TM, TL and OACL on OA joint stability in the CCJ.

Methods: A validated adult FEM was used for injury simulations. The intact normal FEM was reevaluated by comparing the flexionextension ROM data to Panjabi et al (Fig.1) and then was modified to evaluate the sensitivity of the models when damage to TM, TL and OACL were simulated. Injury scenarios evaluated include 1) removal of TM, 2) TL, 3) removal of both TM and TL, 4) OACL injury model (i.e. reducing the stiffness of the OACL by $29.4 \mathrm{~N} / \mathrm{mm}$ ) 5) OACL injury model with TM, 6) TL, 7) both TM and TL removed. Rotations are reported for normal models and percent increases from normal are reported for the injury models.

Results: The flexion-extension ROMs were within one standard deviation of previously reported data (Fig. 1). An isolated injury to the OACL caused the largest increase in flexion ROM (Table 1). The adult FEM with and isolated OACL injury showed a 30.5\% increase in flexion. Isolated TM, TL and TM-TL removal cases increased flexion ROM a maximum of $6 \%$. An OACL injury combined with removal of TL, TM or both further increased flexion ROM from the isolated OACL injury by $<5 \%$.

Conclusion: The results show that the OACL plays a major role in the stability of the CCJ. In contrast to other studies, we found the TM and TL to be relatively small contributors to the stability.

\section{Management of Acute Isolated Atlas Fractures: A Retrospective Study of 65 Patients}

Hyun Su Kim, BS; Kartik Kesavabhotla; Michael Brendan Cloney, BA; Adeola L. Adeoye, APN; Nader S. Dahdaleh, MD

Introduction: Isolated atlas fractures represent 2 to $13 \%$ of acute cervical spine fractures. Their management is usually non-surgical with rigid immobilization and is also dependent on the integrity of the transverse atlantal ligament. We present our experience with the management of 65 patients who presented with acute isolated atlas fractures.
Methods: We retrospectively reviewed the charts of 65 consecutive patients who presented with acute isolated atlas fractures to our institution and have completed at least 3 months of follow up spanning January 2006 and January 2016. The fracture morphology was classified into 5 subtypes (or a combination of) according to Gehweiler et al. Clinical and radiological outcomes were collected as well as mode of treatment.

Results: The average age of the cohort was $54.7 \pm 22.2$. The most common fracture subtype was type III (four point fracture of the anterior and posterior arches) with 22 patients $(33.8 \%)$ followed by type IV (isolated fracture of the lateral mass) with 11 patients $(16.9 \%)$. The overall incidence of associated transverse atlantal ligament disruption was $33.8 \%$ and the incidence of vertebral artery injury was $6.2 \%$. 61 patients were managed successfully with rigid collar bracing; 44 patients $(67.7 \%)$ or crown halovest; 17 patients $(26.2 \%) .4$ patients underwent surgical stabilization (6.2\%). Two of the surgical patients had a type III injury, one had a combined type I and IV, and one had a combined type III and IV injuries. Overall, the fusion rate in our cohort was $83 \%$. All clinical outcomes improved on average including VAS, Frankel, and Nurick scores. On average, some patients exhibited some degree of cranial settling with a decreased basion dental index at the last follow up. However, this was not associated with any clinical symptoms.

Conclusion: Management of acute isolated atlas fractures and its subtypes is primarily non-surgical with rigid immobilization or crown halovest bracing. When associated with transverse atlantal ligamentous injury, non-surgical treatment can be successful. The likelihood for surgical intervention in our cohort was $6.2 \%$.

168. Use of the Acutrak 4/5 Headless Compression Screw for Odontoid Fixation in Type II Odontoid Fractures: A Technical Note and Case Series

Michael Kakareka; Xin Xin; Steven S. Yocom, DO; Alan R. Turtz, MD

Introduction: Type II odontoid fracture is a common cervical spine fracture that disproportionately afflicts the elderly. An Anterior odontoid screw allows immediate fixation and preserves $\mathrm{C} 1 / 2$ motion and is highly effective in fracture healing in acute fractures in patients with anatomically favorable fracture morphology and body habitus. The traditional approach uses a lag screw that invariably disrupts the $\mathrm{C} 2 / 3$ disk to some degree. The Acutrak $4 / 5$ is headless fully threaded variable pitch compression screw that has been described once in the literature to successful treat an odontoid fracture. This is the largest case series on the use of this novel technique that avoid the $\mathrm{C} 2 / 3$ disk space while providing good compression and outcome. Our surgical technique allow for steeper angle of approach as the screw entry is at bottom of body of $\mathrm{C} 2$.

Methods: We retrospectively analyzed eight patients between 2009 and 2014 who underwent an anterior odontoid fixation with an Acutrak 4/5 screw for stabilization of a type II odontoid fracture.

Results: We identified four male and four female patient who underwent surgery during the review period with median age of 71. All patient had at least 3 month follow up except one was lost to follow up at 6 weeks. Inoperatively, Six patients had satisfactory reduction and compression across fracture lines while two patients did not. One patient who had inadequate reduction failed at 6 weeks and required a posterior $\mathrm{C} 1 / 2$ fusion. His fracture had been a 3 month chronic fracture, however. The other seven patients had good radiographic and clinical outcome at last follow up.

Conclusion: Odontoid screw fixation for acute type II odontoid fractures provides high rate of fracture healing and preserved $\mathrm{C} 1 / 2$ motion. The novel use a headless fully threaded variable pitch compression screw such as the Acutrak 4/5 provides comparable outcomes compared to the conventional lag screw, at least in the short term. Long term follow up with larger patient cohort would be 
needed to assess the durability of this technique and potential benefit of reducing adjacent $\mathrm{C} 2 / 3$ disk degeneration.

\section{Clinical Guideline in Managing Peri-Operative Spinal Cord Injury: Results from a Survey of the AOSpine International Community}

Anick Nater, MD, BS; Jean-Christophe Murray; Lindsay Tetreault, Bsc; Aria Nouri, MD, MSc; Allan R. Martin, BASc, MD; Michael G. Fehlings, $M D, P h D, F R C S(C)$, FACS

Introduction: This survey aimed to: 1) evaluate the awareness and usage of clinical guideline(s) and investigate how spine surgeons (2) feel about and manage peri-operative spinal cord injury (SCI) and (3) perceive the value of developing a guideline in the management of peri-operative SCI and their likelihood of using this guideline in their clinical practice.

Methods: Members of the AOSpine International community were invited to participate in an electronic survey related to the awareness, usage of guideline(s) and demographics; staff surgeons and surgical residents performing spine surgery were presented with two clinical scenarios describing peri-operative SCI. Results from the entire sample were analyzed, as well as differences between respondents, such as specialty, geographic regions, level of training and comfort.

Results: Of the 770 respondents, $85.6 \%$ reported being aware of the existence of guideline(s) and 79.0\% acknowledged using such guideline(s). Among 485 staff surgeons with at least $50 \%$ of their practice dedicated to spine surgery, of which $67.4 \%$ have completed a spine fellowship and $83.9 \%$ have more than 5 years of practice, (i) $53.6 \%$ reported not feeling comfortable managing a patient who wakes up quadraplepic after an uneventful multi-level posterior cervical decompression with instrumented fusion; $22.3 \%$ would consider an immediate return to the operating room while $77.7 \%$ would either obtain an MRI (67.6\%), administer high-dose steroids $(40.2 \%)$ or increase the mean arterial pressure (37.7\%) first; (ii) $57.5 \%$ reported not feeling comfortable managing a patient who had a complete loss of lower extremities somatosensory and motor evoked potentials after closing a pedicle subtraction osteotomy; $66.8 \%$ considered the release of the correction as the most important intervention to maximize recovery of this potential SCI. Among these staff surgeons, $90.3 \%$ believed that a guideline in the management of peri-operative SCI would be useful and $93.8 \%$ would be either likely or extremely likely to use this guideline.

Conclusion: The majority of respondents are aware and routinely use clinical guideline(s) in their practice. Despite considerable training and experience, most staff spine surgeons reported not feeling comfortable managing peri-operative SCI. However, they highly value the creation and are likely to use a guideline in the management of peri-operative SCI.

\section{Indication of Anterior Decompression in Complex Irreducible Atlanto Axial Dislocation}

\section{Arun K. Srivastava, MCh}

Introduction: This retrospective study attempt to know the variables responsible for unsatisfactory clinico-radiological outcome following posterior decompression in cases of complex irreducible atlanto axial dislocation (AAD).

Methods: Total 108 patients of complex irreducible AAD, operated by the single surgeon over 3 years with minimum of six month follow up were retrospectively analysed. Ventral decompression following posterior decompression was performed in 24 patients. Japanese Orthopaedic Association Score modified for Indian patients
(mJOAS) in both preoperative and postoperative period was used for myelopathy grading.

Results: In thirteen patient (54\%) radiological proven incomplete reduction observed; Nine patients $(37 \%)$ had radiological satisfactory reduction without any improvement or deterioration in the Myelopathy; Two patients $(8 \%)$ of irreducible Os odontoideum required anterior decompression in same sitting .The significant variable associated with failed posterior approach was vertebral artery(VA) anomaly in the third part with more than 70 degree saggital orientation of $\mathrm{C} 1 / \mathrm{C} 2$ joint and Platybasia of severe degree. Soft tissue compression was associated with three cases. The variables like Klippel feil anomaly $[\mathrm{n}=14]$, Os odontoideum [n=2], BI $(\mathrm{n}=14)$, assimilated atlas $(\mathrm{n}=24)$, lateral mass of $\mathrm{C} 1$ asymmetry $(\mathrm{n}=14)$ articular mass of $\mathrm{C} 2$ asymmetry $(n=14)$, rotational component $(n=15)$, platybasia $(n=11)$ were analyzed. Mean ECD in irreducible AAD was $(7 \pm 2.7)$ in mm.14 patients had VA variations at $3 r d$ part at $\mathrm{CV}$ junction. Compare to Pre op MJOA score, mild $(n=1)$, moderate $(n=8)$ or severe $(n=15)$, in postoperative period majority of them shown some improvement in myelopathy in postoperative period. MJOA score [mild $(n=1)$; moderate $(n=14)$; severe $(\mathrm{n}=9)]$.

Conclusion: The Saggital angle of $\mathrm{C} 1 / \mathrm{C} 2$ joint more than 70 degree along with 3rd part VA anomaly is the significant factor limiting the joint manipulation and satisfactory reduction during the Posterior approach and thus warranting anterior decompression in this subset of patient.

171. Long-term Clinical Outcomes Following Surgical Management of Cervical Spine Fractures in Elderly Patients

Nitin Agarwal, MD; James Zhou, BS; Gurpreet Surinder Gandhoke; Michael Koltz, MD; Adam S. Kanter, MD; David O. Okonkwo, MD, $P h D ;$ D. Kojo Hamilton, $M D$

Introduction: The proper management of cervical spine fractures in the elderly population is a controversial issue. To date, no studies have compared the outcomes of surgical management of cervical fractures in elderly patients aged 65 to 74 to those of elderly patients 75 years of age and older. Given the possibility of increased frailty in "older" elderly patients (i.e. 75 years of age and older), it is important to consider how the overall short-term and long-term outcomes of surgery in patients 75 and older compare to those of patients under the age of 75. In this study, we aim to determine if the outcomes of surgical management differ when comparing patients $=75$ years old to patients aged 65 to 74 .

Methods: A retrospective review was conducted of a prospectively maintained database of 107 elderly patients who experienced cervical spine trauma between 2007 and 2014. Of these patients, 46 patients were managed surgically. 19 of the surgically managed patients were 65 to 74 years old; the remaining 27 surgically managed patients were 75 years old or greater. Chi-square analysis and Fisher exact tests were performed to the two patient groups on various outcome measures, include length of stay, hospital mortality, follow-up mortality, improvement in neurologic status, and the development of mental status decline in the follow-up period.

Results: Based on our analysis, there are no statistically significant differences in a number of clinical outcomes when comparing patients $<75$ and patients $=75$ in a number of clinical outcome measures. Tested measures include length of stay, likelihood of death during admission, likelihood of death following discharge, likelihood of developing mental status decline following discharge, post-operative neurologic improvements, and long-term functional status.

Conclusion: Patients aged 75 and older have statistically similar outcomes following surgical management of cervical spine fractures when compared to patients under the age of 75 . These results indicate that surgical management of cervical fractures in elderly patients is beneficial without regard to age, and should not be withheld for fear of poor outcomes in "older" elderly patients. 
172. Thoracolumbar Fractures in the Elderly: Complications After Non-Operative and Operative Treatment

Taylor Elise Purvis, BA; Rafael De la Garza-Ramos, BA; C. Rory Goodwin, MD, PhD; Daniel M. Sciubba, MD

Introduction: Although thoracolumbar fractures make up approximately ninety percent of all spine fractures, the optimal treatment is unknown. Management of these fractures in elderly patients, who present with higher rates of comorbidities and lower rates of osseous union, has not been adequately explored in the spine literature. We sought to compare in-hospital outcomes for operative versus non-operative treatment of patients over 75 years of age with thoracolumbar fractures using a national database.

Methods: The 2002-2011 NIS database was queried for patients over the age of 75 with a primary diagnosis of thoracolumbar fracture without spinal cord injury. Patients with an associated diagnosis of vertebral column tumor were excluded. The sample was stratified based on treatment modality as non-operative, operative (spinal decompression and/or fusion) and vertebroplasty/kyphoplasty (VP/ $\mathrm{KP})$. Variables analyzed included development of at least one in-hospital complication, length of stay, and total adjusted charges.

Results: A total of 61,119 patients undergoing treatment for thoracolumbar fracture were identified; 1,520 treated operatively, 48,143 treated non-operatively, and 11,456 treated with VP/KP. Patients treated operatively had the longest in-hospital stays $(8.7 \pm$ 8.3 days vs. $5.0 \pm 4.2$ days [non-operative] and $5.2 \pm 4.2$ days [VP/ $\mathrm{KP}]$; $\mathrm{P}<0.001)$. Adjusted charges were highest for operative patients $(\$ 123,499 \pm 135,406$ vs. $\$ 27,261 \pm 32,703$ [non-operative] and $\$ 42,642$ $\pm 33,958$ [VP/KP]; $\mathrm{P}<0.001$ ). Operative patients had the highest rates of developing at least one complication (16.2\%), versus $8.7 \%$ in the non-operative and $8.2 \%$ in the $\mathrm{VP} / \mathrm{KP}$ groups $(\mathrm{P}<0.001)$. Patients with operative treatment had the highest percentages of unplanned intubation, myocardial infarction, pneumonia, stroke, pulmonary embolism, shock, acute respiratory distress syndrome (all $\mathrm{P}<0.001$ ) and acute kidney injury $(\mathrm{P}=0.038)$.

Conclusion: Clinicians should advise elderly patients of the potential increased risk associated with operative treatment for thoracolumbar fractures. Future research is necessary to compare more long-term complications among treatment groups.

\section{Posterior Thoracolumbar Corpectomy and Reconstruction with Two Small Cages. Technical Note and Clinical Study}

\section{Jae-Young Park, MD; Jung-Kil Lee, MD, PhD}

Introduction: The combined posterior-anterior approach has been widely employed for single level corpectomy in destructive thoracolumbar spinal disease. However, anterior corpectomy and fixation is technically demanding and has several disadvantages. Therefore, we tried the posterior approach only for decompression and circumferential reconstruction.

Methods: From July 2013 to Dec. 2015, ten consecutive patients were treated at our institution using this technique in various spinal disease including burst fracture, osteoporotic compression fracture and deformity. After performing subtotal or total corpectomy with upper and lower discectomy were performed, 360-degree reconstruction with two small titanium mesh cages insertion and correction of kyphosis by posterior transpedicular screw fixation were performed. Clinical and radiological data were retrospectively analyzed.

Results: All ten patients ( 2 male and 8 female, mean age: 68.2 years) suffered from severe kyphotic deformity with or without neurological deficits. Mean surgical time was 374 minutes. Mean blood loss was $1220 \mathrm{~mL}$. All patients experienced pain relief after the procedure. There was no intraoperative complication and newly developed neurological deficit after surgery. A Successful restoration for kyphotic change was achieved in all patients and maintained during follow-up period.

Conclusion: This operation is a reliable, effective, safe and less invasive treatment option and can be a good alternative modality for various spinal diseases. Long-term follow-up study with large number will be required to clarify the effectiveness of this technique in the future.

174. Surgical Practice in Traumatic Spinal Fracture Treatment With Regard to the TLICS and SLICS Systems: A Review of 58 Patients at the University of Wisconsin

Kimberly Michelle Hamilton, MD; Darnell T. Josiah, MS, MD; Nathaniel P. Brooks, MD

Introduction: Spine surgeons at a Level 1 Trauma Center have observed a high incidence of spine and spinal cord injuries due to falls from tree stands during the hunting season. These injuries have been retrospectively reviewed in the context of the TLICS and SLICS classification systems to assess the inter-user reliability and validity.

Methods: We reviewed the University of Wisconsin Hospital and Clinics' trauma database for tree-stand-related injuries from 1999 to 2013, and selected for patients suffering from spine and spinal cord injuries. We collected data pertaining to hunters' demographics, comorbidities, type and mechanism of injury, injury severity and Glasgow outcome scores. Independent reviewers, who were blinded to the patients' treatments, reviewed the spinal imaging, and assigned scores according to the TLICS and SLICS scale. We then analyzed the inconsistencies between reviewers, and between the suggested treatment and our institution's management practices.

Results: The two reviewers assigned identical scores in only 25 of 67 cases $(37 \%)$. While exact scores were infrequently identical, the assignment to operative or conservative management was consistent between reviewers in 14/15 (93\%) of cervical and 47/52 (90\%) of thoracolumbar scores. With the scores averaged between reviewers, the score-assigned treatment was consistent with the actual treatment in 11/15 (73\%) cervical cases and 44/52 (85\%) thoracolumbar cases.

Conclusion: The TLICS and SLICS systems were reflective of our surgeons' treatment strategies in the majority of cases within this trauma-based patient population. Classification systems should be used with caution in the setting of multi-trauma as the patient's injuries may in fact be more complex than suggested by the TLICS or SLICS score. Systems such as TLICS and SLICS may be best applied in the educational setting to confirm between surgeons and radiologists the complex fracture morphology and presence of ligamentous injury.

\section{Prevention of Iatrogenic Cervical Cord Injury: Preoperative Screening}

Stephen Shelby Burks, BA, MD; Jason Liounakos, MD; David McCarthy; Howard B. Levene, MD, PhD

Introduction: A rare but devastating complication of surgery / general anesthesia is iatrogenic cervical cord injury. Anesthesia providers have called for better understanding and prevention as review of claims data demonstrated these to be particularly costly instances. We present a series of patients where Neurosurgical evaluation of the cervical spine was requested, preoperatively, in those complaining of neck pain, where providers appreciated symptoms of myelopathy, or CSM was appreciated on preoperative images. Patients were evaluated retrospectively to determine whether preoperative screening measures introduced changes in management.

Methods: Data collected from 2010 to 2016 at the Miami VA Hospital. Standardized, preoperative cervical screening consisted 
of questioning for signs of myelopathy, as well as detailed physical exam including reflexes, strength, sensation, etc. Physical exam included cervical range of motion with attention on neck extension held for 1 minute. Positive finding being new sensory abnormalities in extension or at baseline.

Results: Forty patients were referred for cervical evaluation and clearance. Predominant referring service was Otolaryngology (19). Objective signs of myelopathy were noted in 8 patients. Using neck extension, sustained for 1 minutes, we elicited sensory abnormalities in 4 additional patients. A total of 18 patients had significant changes to their surgical plan; use of SSEP monitoring (12), transition to percutaneous surgery (1), plan for surgical decompression (1), and cervical decompressive surgery (4).

Conclusion: This particular series represents a guideline to the management of potentially high risk cervical patients. All examinations were supplemented with a prolonged neck extension maneuver as part of the Range of Motion exam. With our screening measures we aimed to detect subtle forms of myelopathy and found a significant portion of patients with positive findings. These basic screening methods could easily be employed by anesthesia, or other, personnel preoperatively and could potentially avoid the complication of iatrogenic spinal cord injury.

176. Early Surgical Intervention for Acute Central Cord Syndrome is not Associated with Increased Mortality

Jakub Godzik, MD; Jay Dalton; Rohit Mauria, BS; Alan Cook; Kristina Chapple, PhD; Jay D. Turner, MD, PhD

Introduction: The influence of surgical timing for acute central cord syndrome (ACS) on patient mortality is controversial. Recent reports have conflicting results. The objective of this study was to evaluate the impact of early versus delayed surgical intervention on inpatient mortality using propensity score weighting to control for confounding factors not addressed in prior studies.

Methods: We performed a retrospective study using the National Trauma Data Bank (NTDB, 2011-2014), including patients $>18$, with ACS (identified using ICD-9 coding). Information was collected on demographics, mechanism of injury, surgical timing ( $<24$ hours, $>24$ hours), Charlson Comorbidity index (CCI), injury severity index (ISS), and mortality. Logistic regression and propensity weighting were used to evaluate relationship between hospital mortality and surgical timing.

Results: A total of 2383 patients with ACS following trauma were identified. Average age was $56 \pm 15$, and $79.3 \%$ were male, with an average ISS of $19.5 \pm 9.0$ and mortality rate of $3.0 \%$. A total of 731 patients underwent surgery for ACS within 24 hours. Univariate analysis did not demonstrate a significantly higher mortality rate in the early versus late surgery groups $(3.8 \%$ vs $2.7 \%, p=0.127)$, although the early surgery group demonstrated significantly higher ISS $(p=0.04)$, lower CCI $(p<0.001)$, and younger age $(p<0.001)$. Predictors of death in the early surgery group included older age $(p<0.001)$, higher CCI $(p<0.001)$, higher ISS $(p<0.001)$, and GCS $<15$ $(\mathrm{p}=0.021)$. Logistic regression demonstrated higher ISS (OR 1.05, $\mathrm{p}<0.001$ ), higher CCI (OR 1.84, $\mathrm{p}<0.001$ ), and days to surgery (OR $0.89, \mathrm{p}=0.027$ ) as significant predictors of mortality. Propensity score weighting demonstrated no significant relationship between days to surgery and in-hospital mortality $(\mathrm{P}=0.138)$.

Conclusion: Delayed surgical intervention does not appear to reduce mortality among patients with acute central cord syndrome. We propose that survival in the NTDB is confounded by multisystem trauma and existing comorbidities, rather than choice of surgical timing.
177. Trends in Patient Care for Traumatic Spinal Injuries in the United States: A National Inpatient Sample Study of the Correlations With Patient Outcomes From 2001 to 2012

Marcus D. Mazur, MD; Christopher M. Holland, MD, PhD; Erica F. Bisson; Meic H. Schmidt, MD; Andrew T. Dailey, MD

Introduction: Although patient outcomes after cranial injuries are better at high-volume centers with specialized, multidisciplinary teams, similar assessments have not been done for spinal injuries. We examined changes in patient characteristics, distribution of care, and patient outcomes for spinal cord injury (SCI) in the US between 2001 and 2012.

Methods: We retrospectively reviewed the National and Nationwide Inpatient Samples for the years 2001, 2002, 2011, and 2012 to identify patients with spinal fracture with or without SCI. The demographic characteristics of the patient cohort, clinical course, hospital characteristics, interhospital transfer, and disposition were statistically analyzed relative to patient mortality, total hospital costs, and length of stay. How these data changed over this 11-year period was also evaluated.

Results: A total of 159,875 cases were identified, with 141,737 fractures without SCI and 18,138 SCIs with or without fracture. There was a statistically significant decrease in the percentage of patients transferred with spine injury from $4.2 \%$ to $3.4 \%(\mathrm{p}<0.001)$ from the early years to the later years and in patient transfers for SCIs $(8.1 \%$ versus $6.5 \%, \mathrm{p}<0.001)$. Interestingly, the overall mortality rate $(3.5 \%$ v $3.6 \%)$ remained unchanged $(\mathrm{p}=.679)$, but mortality from SCI increased $(6.6 \%$ to $7.4 \%, \mathrm{p}=.021)$.

Conclusion: From 2002 to 2012, the rate of interhospital transfer of spinal injury patients declined, while the mortality rate for patients with SCI increased. Interestingly, there was an increase in transfers after operative spinal surgery at the index hospital. The decentralization of spine care may be responsible for the increase in mortality.

178. Pathologic Position of the Transverse Ligament Prevents Reduction in Fixed, Irreducible Os Odontoideum Operative Illustration and Radiographic Correlate in $\mathbf{4 1}$ Patients

Brian J. Dlouhy MD; Bruno Policeni; Arnold H. Menezes, MD

Introduction: Os odontoideum $(\mathrm{OO})$ is a craniovertebral junction (CVJ) abnormality in which an ossicle (small bone) is cranial to a hypoplastic dens by a variable gap. OO can result in instability, which may be reducible or irreducible. What leads to irreducibility in $\mathrm{OO}$ is unclear. Therefore, we sought to better understand the causes of irreducibility in OO.

Methods: We conducted a retrospective review, which identified more than 200 patients who had undergone surgical treatment for OO from 1978 to 2015 at the University of Iowa Hospitals \& Clinics. Only the 41 patients found to have irreducible $\mathrm{OO}$ were included in this study. All inpatient and outpatient records were retrospectively reviewed.

Results: Forty-one adults and children were found to have irreducible OO. A form of atlantoaxial dislocation was seen in all cases. On CT, in all 41 cases, atlantoaxial facets were dislocated but did not have osseous changes that would have prevented reduction. On MRI, the transverse ligament was identified anterior and inferior to the ossicle and superior to the hypoplastic odontoid process in all cases in which MRI was available (post MRI era - 36 of 36 cases). It was hypointense on $\mathrm{T} 2$ but also had an associated hyperintense signal on T2. Intraoperatively, the transverse ligament was identified anterior and inferior to the ossicle and superior to the hypoplastic odontoid process in all 41 cases.

Conclusion: In the largest series to date of irreducible $\mathrm{OO}$ and the only study to examine variable factors that lead to irreducibility 
in $\mathrm{OO}$, we found that the position of the transverse ligament anterior and inferior to the os is the most common factor in the irreducibility of OO. This is the first study in which intraoperative findings of the transverse ligament have been correlated with MRI.

179. Short Segment Posterior Fixation with Index Level Screws Versus Long Segment Posterior Fixation for Thoracolumbar Spine Fracture: Angle of Correction and Pain

Walid Ahmed Abdel Ghany, MD, PhD; Ahmed Mamdouh Sallam, MBBS, MS; Mohamed A. Habib; Ali Kotb Ali; Sherif H. Abo Zied; Mohamed S. Ismail; Ahmed M. Elsayed; Ahmed F. Toubar

Introduction: Traumatic spinal fractures of the thoracolumbar area represent approximately $90 \%$ of all spinal fractures. mostly unstable, require surgical intervention. its management has been controversial between advocates of long segment fixation versus short segment fixation

Methods: A prospective study of 61 patients,admitted to Ain Shams University and Arab Contractors Co Hospitals have single level thoracolumbar spine fracture with Cobb's angle $=25^{\circ}$, underwent posterior fixation. Of them, Thirty three patients underwent short segment fixation one level above and one level below with screws into the index level, and twenty eight patients underwent long segment fixation with two levels above and two levels below with skipped index level. The angle of correction and pain were regularly assessed by Cobb's angle measurement and visual analogue scale (VAS) respectively.

Results: The sixty one patients who underwent posterior fixation , 33 short segment cases (54.1\%) and 28 long segment cases (45.9\%). All were followed for 12 months. In short segment group the preoperative mean Cobb's angle was $19.3^{\circ} \pm 3.7^{\circ}$,whereas, in long segment group the pre-operative mean Cobb's angle was $18.6^{\circ} \pm 3.8^{\circ}$ $(\mathrm{P}=0.520)$. the post operative mean angle of correction were $6.8^{\circ}$ $\pm 2.6^{\circ}$ and $5.8^{\circ} \pm 1.6^{\circ}$ respectively $(\mathrm{P}=0.098)$. After 1 year follow up, the angle of correction have become $7.8^{\circ} \pm 1.6^{\circ}$ and $7.9^{\circ} \pm 1.8^{\circ}$ respectively $(\mathrm{P}=0.860)$. The pain was assessed by VAS on regular base follow up. In short segment group the pre-operative VAS was 5.6 \pm 2.1 whereas the long segment group VAS was $5.1 \pm 2.1(\mathrm{P}=0.284)$. After 6 months the VAS were $2.9 \pm 1.4$ and $3.9 \pm 1.4(\mathrm{P}=0.883)$ respectively. On one year follow up the VAS were $1.4 \pm 0.5$ and $1.8 \pm$ $0.4(\mathrm{P}=0.590)$ respectively.

Conclusion: There is no superiority of long segment over short segment fixation with index level stabilization in correction of kyphotic angle. Moreover, short segment fixation has faster relief of pain.

\section{The Relationship Between Mortality and Acute Spinal Cord Injury}

Chengmin Zhang, PhD; Fred H. Geisler, MD, PhD; Karen K. Anderson, BS; Qiang Zhou, MD; Paul M. Arnold, MD

Introduction: Surgical decompression can facilitate recovery in SCI patients; still unknown is the relationship between mortality and specific SCI factors. We analyzed mortality and SCI in relation to level and grade of injury, age and gender to delineate demographic or neurological trends.

Methods: Retrospective review of 43 patients who died during the Sygen $\AA$ trial, a randomized multicenter study assessing the influence of GM-1 ganglioside on neurologic recovery after SCI. Age, gender, fracture level, and cause and severity of injury were analyzed. Patients were divided by age $(=29$ years, $=30$ years $)$ and injury level and severity.

Results: Of 760 patients, 43 died within one year (mortality $5.66 \%$ ). Of 43 deaths, 30 (27 male) underwent surgery; 13 (9 male) did not. Of 43 deaths, $37(86.05 \%)$ were cervical and $6(13.95 \%)$ were thoracic. Of
579 cervical injuries, 37 (6.39\%) were fatal; of 181 thoracic, $6(3.31 \%)$ were fatal. Of 760 patients, $37(4.87 \%)$ with cervical injuries died, compared to $6(0.79 \%)$ thoracic. Of 43 deaths, 16 (37.21\%) were C4; 8 (18.60\%) C5; 5 (11.63\%) C7; 3 (6.98\%) each C3 and T8. Of 43 deaths, $34(79.07 \%)$ were Grade A; $4(9.3 \%) \mathrm{B}$, and $5(11.63 \%)$ Grade C+D. Of 482 Grade A patients, 34 (7.05\%) died; of 131 Grade B, $4(3.05 \%)$; and of 147 Grade C+D, 5 (3.40\%). Of 760 patients, 34 (4.47\%) Grade A died; 4 (0.53\%) Grade B; and $5(0.66 \%)$ Grade C+D. In 30 surgical mortality patients (24 Grade A), $11(36.67 \%)$ at $\mathrm{C} 4$ died; $7(23.33 \%)$ at C5; and $3(10 \%)$ each C7 and T8. In 13 non-surgical mortality patients (10 Grade A), $5(38.46 \%)$ at $\mathrm{C} 4$ and $2(15.38 \%)$ at $\mathrm{C} 7 \mathrm{died}$.

Conclusion: Surgical decompression can decrease mortality and prolong patients' lives, especially for patients with cervical AIS Grade A SCI.

\section{Application of AOSpine Subaxial Cervical Spine Injury Classification in Simple and Complex Cases}

Bizhan Aarabi, MD; Cumhur Oner; Alexander R. Vaccaro, MD; Gregory Schroeder; Noori Akhtar Danesh

Introduction: Cervical spine injury classification systems should be simple, easy to relate and remember, reliable guide for surgical planning and predictor of outcome in clinical settings. We investigated whether the AOSpine subaxial cervical spine classification system predicted injury severity and neurologic outcome.

Methods: We analyzed the relevant clinical, imaging, management, and AIS grade conversion of 92 AIS grades A-C patients with cervical spine injury. We correlated morphology class with age, injury severity score (ISS), follow-up ASIA motor score (AMS), intramedullary lesion length (IMLL), and ASIA impairment scale (AIS) grade conversion at 6 months post injury.

Results: The mean age of patients was 39.3 years, 83 were male, and 69 were injured during an automobile accident or following a fall. The AOSpine class was A4 in 8, B2 in 5, B2A4 in 16, B3 in 19, and $\mathrm{C}$ in 44 patients. The mean ISS was 29.7 and AMS was 17.1. AIS grade was $\mathrm{A}$ in 48, B in 25, and $\mathrm{C}$ in 19 patients. Mean IMLL on postoperative MRI was $72 \mathrm{~mm}$ : $\mathrm{A} 4=68.1$; $\mathrm{B} 2 \mathrm{~A} 4=86.5$; $\mathrm{B} 2=59.3$; $\mathrm{B} 3=46.8$; and $\mathrm{C}=79.9$. At a mean follow-up of 6 months, the mean AMS was 39.6. Compared to patients with class B3 injuries, those with class $C$ injuries were significantly younger $(\mathrm{P}<0.0001)$, had longer IMLL $(\mathrm{P}<0.002)$, and were less likely to have AIS grade conversion to a better grade $(\mathrm{P}<0.02)$.

Conclusion: The AOSpine subaxial cervical spine injury classification system successfully predicted injury severity (longer IMLL) and chances of neurologic recovery (AIS grade conversion) across different class subtypes.

182. A Clinical Practice Guideline for the Management of Patients with Acute Spinal Cord Injury: Recommendations on the Role of Baseline Magnetic Resonance Imaging in Clinical Decision Making and Outcome Prediction

Allan R. Martin, BASc, MD; Bizhan Aarabi, MD; Paul A. Anderson, MD; Paul M. Arnold, MD; Darrel S. Brodke, MD; Anthony Burns, MD; Kazuhiro Chiba; Joseph Dettori; Julio C. Furlan, MD, PhD, MBA, MSc; James S. Harrop, MD, FACS; Langston T. Holly, MD; Susan Howley; Tara Jeji; Sukhvinder Kalsi-Ryan BScPT, MSc, PhD; Mark Kotter; Shekar N. Kurpad, MD, PhD; Brian K. Kwon, MD, PhD, FRCSC; Ralph Marino; Eric M. Massicotte, MD; Geno Merli; James Middleton; Hiroaki Nakashima, MD; Narihito Nagoshi, MD, PhD; Katherine Palmieri; Anoushka Singh; Andrea Skelly, PhD; Lindsay Tetreault, Bsc; Jefferson R. Wilson, MD, PhD; Albert Yee, MD; Michael G. Fehlings, MD, PhD, FRCS(C), FACS

Introduction: Magnetic resonance imaging (MRI) is the gold standard for imaging the spinal cord and related soft tissues; however, 
there remains debate about the appropriate use of MRI in patients with acute spinal cord injury (SCI) as it requires considerable resources and may be risky in trauma patients with respiratory difficulties or hemodynamic instability. This guideline aims to outline the role of MRI in clinical decision making and outcome prediction in patients with traumatic SCI.

Methods: A systematic review of the literature was conducted to address the following key questions and inform guideline development: (1) how does the acquisition of a baseline MRI influence management strategy(ies) and, consequently, neurologic, functional, patient-reported and safety outcomes?; (2) do spinal cord lesion characteristics, pattern and length identified on baseline MRI predict neurologic, functional, patient-reported, and safety outcomes?; (3) do spinal cord characteristics identified on diffusion tensor imaging (DTI) predict neurologic, functional, patient-reported and safety outcomes?; (4) is there evidence to suggest that baseline MRI is cost-effective in patients with acute SCI? A multidisciplinary guideline development group (GDG) used this information, in combination with clinical expertise and patient input, to develop recommendations on the use of MRI in the evaluation and treatment of patients with SCI.

Results: Based on the limited available evidence and the clinical expertise of the GDG, our recommendations were: (1) "We suggest that MRI be performed in adult patients with acute spinal cord injury prior to surgical intervention, when feasible, to facilitate improved clinical decision-making" and (2) "We suggest that MRI should be performed in adult patients in the acute period following SCI, before or after surgical intervention, to improve prediction of neurologic outcome."

Conclusion: These guidelines should be implemented into clinical practice to improve outcomes and prognostication for patients with SCI.

183. A Clinical Practice Guideline for the Management of Patients with Acute Spinal Cord Injury: Recommendations on the Type and Timing of Rehabilitation

Anthony Burns, MD; Bizhan Aarabi, MD; Paul A. Anderson, MD; Paul M. Arnold, MD; Darrel S. Brodke, MD; Kazuhiro Chiba; Joseph Dettori; Julio C. Furlan, MD, PhD, MBA, MSc; James S. Harrop, MD, FACS; Langston T. Holly MD; Susan Howley; Tara Jeji; Sukhvinder Kalsi-Ryan BScPT, MSc, PhD; Mark Kotter; Shekar N. Kurpad, MD, PhD; Brian K. Kwon. MD, PhD, FRCSC; Ralph Marino; Allan R. Martin, BASc, MD; Eric M. Massicotte, MD; Geno Merli; James Middleton; Hiroaki Nakashima, MD; Narihito Nagoshi, MD, PhD; Katherine Palmieri; Anoushka Singh; Andrea Skelly, PhD; Lindsay Tetreault, Bsc; Jefferson R. Wilson, MD, PhD; Albert Yee, MD; Michael G. Fehlings, MD, PhD, FRCS(C), FACS

Introduction: Rehabilitation plays a central role in maximizing function and facilitating community reintegration following a spinal cord injury (SCI). Despite this, many fundamental questions remain regarding the timing and efficacy of various rehabilitation strategies. The objective of this study is to develop guidelines that outline the appropriate type and timing of rehabilitation in patients with acute SCI.

Methods: A systematic review of the literature was conducted to address the following questions: (1) Does the time interval between injury and commencing rehabilitation affect outcome? (2) What is the comparative effectiveness of different rehabilitation strategies? (3) Are there patient or injury characteristics that impact the efficacy of rehabilitation? (4) What is the cost-effectiveness of various rehabilitation strategies? A multidisciplinary guideline development group used this information, in combination with their clinical expertise, to develop recommendations for the type and timing of rehabilitation. The benefits and harms, financial impact, acceptability, feasibility and patient preferences of each recommendation were carefully considered.
Results: (1) We suggest rehabilitation be offered to patients with acute spinal cord injury when they are medically stable and can tolerate required rehabilitation intensity; (2) We suggest BWSTT as an option for ambulation training in addition to conventional overground walking, dependent on resource availability, context, and local expertise; (3) We suggest that individuals with acute and subacute cervical SCI be offered FES as an option to improve hand and upper extremity function; and (4) Based on the absence of any clear benefit, we suggest not offering additional training in unsupported sitting beyond what is currently incorporated in standard rehabilitation.

Conclusion: These guidelines should be implemented into clinical practice to improve outcomes and reduce morbidity in patients with SCI by promoting standardization of care, decreasing the heterogeneity of management strategies and encouraging clinicians to make evidence-informed decisions.

184. Variations of Lumbopelvic Alignment in Standing, Seated, and Slumped Postures in a Cohort of Asymptomatic Adults: Implications for Lumbar Fusion Surgery

Avinash G. Patwardhan, PhD; Fouad Jabbour; Robert Havey; Saeed Khayatzadeh, PhD; Jean-Charles LeHuec; Ellis Cohen; Laurie M. Lomasney; Antonio Faundez; Leonard I. Voronov, MD, PhD

Introduction: Lower lumbar spine is the most prevalent site of spinal fusions for painful degenerative conditions in adults. Lumbopelvic alignment in the standing posture is recommended as the ideal fusion alignment to avoid postoperative complications even though adults spend increasingly larger amount of time sitting. In this study we asked: how do sitting postures alter the lower lumbar spine alignment?

Methods: Full-length standing lateral radiographs of 11 asymptomatic subjects [39 years (SD: 12$), 10 \mathrm{M} / 1 \mathrm{~F}]$ were analyzed. Each subject was instructed to assume the following postures: (i) standing erect $(\mathrm{P} 1)$, (ii) sitting erect (P2), and (iii) sitting slumped (P3). Subjects were instructed to maintain horizontal gaze while a full-length lateral radiograph was taken for each posture. Lumbopelvic alignment parameters were compared between three postures (P1 vs P2 vs P3).

Results: L4-S1 lordosis significantly decreased (28.8 to 17.4 degrees, $\mathrm{p}<0.001)$ when transitioning from standing (P1) to sitting erect $(\mathrm{P} 2)$. Postural change from erect sitting to slumped sitting (P2 to P3) caused significant change in L1-L3 alignment, from 11.1 degrees of lordosis to 3.1 degrees of kyphosis $(p<0.001)$. Figure shows a subject with the average alignment parameters of the cohort. The yellow overlay demonstrates spinal alignment (without other compensation) if the L4-S1 segment were fused in lordotic alignment corresponding to standing erect posture (P1). Because the L4-S1 alignment cannot change after fusion, the L4 superior endplate along with the rest of the cephalad segments are posteriorly tilted in the erect seated posture. The alignment mismatch is further exacerbated in the slumped posture.

Conclusion: L4-S1 lordosis significantly decreased when transitioning from standing to sitting. If fusion across L4-S1 is indicated, our observations suggest the sagittal alignment of the lower lumbar spine in the standing posture should not be considered as the gold standard for surgical reconstruction since it may put the proximal segments at risk of developing postfusion breakdown. 


\section{Spinal Deformity Surgery in Patients with Movement Disorders}

Rafael De la Garza-Ramos, BA; C. Rory Goodwin, MD, PhD; Benjamin D. Elder, MD, PhD; Taylor Elise Purvis, BA; Amit Jain, BS; Alejandro Ruiz-Valls; A. Karim Ahmed; Daniel Martinez-Ramirez, MD; Isaac O. Karikari, MD; Daniel M. Sciubba, MD

Introduction: The objective of this study is to investigate the immediate postoperative morbidity rate of patients with movement disorders (MD) after spinal deformity surgery.

Methods: The Nationwide Inpatient Sample database from 2002 to 2011 was queried to identify adult patients with MD who underwent spinal deformity surgery. Complication rates were compared between patients with MD and controls. A multiple logistic regression analysis was conducted to assess the effect of MD on outcome.

Results: Three-hundred and sixty-five patients with MD (3.3\%) were identified among 11,043 patients undergoing surgery for spinal deformity. Patients with MD were on average 8 years older than the control group (67 vs. 59 years of age, $\mathrm{p}<0.001$ ). The complication rate was $55.1 \%$ for patients with MD and $43.7 \%$ for patients without MD $(\mathrm{p}<0.001)$. The most common complication was acute post-hemorrhagic anemia, which occurred in $31.9 \%$ of all patients $(41.6 \%$ in MD patients and $31.5 \%$ in the control group, $\mathrm{p}<0.001)$. Other complications that were more common in patients with MD included delirium $(\mathrm{p}<0.001)$, acute kidney injury $(\mathrm{p}=0.032)$, and pulmonary embolism $(\mathrm{p}=0.014)$. After controlling for patient age, sex, osteoporosis, complex procedures, fusion to the lumbosacral spine, use of bone morphogenetic protein, and use of blood transfusion, patients with MD were 1.3 times more likely to develop a complication compared to patients without MD (OR 1.27; 95\% CI, $1.021 .59 ; \mathrm{p}=0.032$ ) on multiple logistic regression analysis.

Conclusion: Patients with MD who undergo spinal deformity surgery may have worse short-term outcome compared to patients without these disorders.

\section{Spine Deformity Surgery in the Elderly: Risk Factors and 30-day Outcomes}

John K. Yue, BA; David Sing; Sourabh Sharma; Pavan S. Upadhyayula, BA; Lionel N. Metz, MD

Introduction: Spine deformity surgery rates have increased dramatically over the past 2 decades, with the highest increases seen in the elderly (over 60-years). In a population with relatively higher comorbidities, the risk factors for poor outcome following elective spine fusion remain in need of improved characterization.

Methods: Patients aged over 60-years who underwent elective posterior or anterior-posterior ("combined") fusion were extracted from the American College of Surgeons National Surgical Quality Improvement Program (ACS-NSQIP) database years 2007-2013 and analyzed by surgical cohort (posterior-vs.-combined). Primary outcome measures include operation time, hospital length of stay (HLOS), 30-day complications, and discharge destination. Multivariable logistic and linear regressions controlling for demographic/clinical variables were performed for outcomes. Odds ratios (OR), mean differences (B) and respective 95\% confidence intervals $(\mathrm{CI})$ are reported.

Results: Overall, 881 elderly subjects underwent fusion for spine deformity (18.2\%-combined; $81.8 \%$-posterior). Subjects were aged $70 \pm 6.2$ years, $32.8 \%$ male, and $87.2 \%$ Caucasian. Posterior fusions were associated with extreme body habitus (obese class II/ III and underweight; $p=0.027)$, functional independence $(97.5 \%$-vs.$91.8 \%$; $\mathrm{p}=0.010$ ), and multilevel fusions (7-12 levels: $24.8 \%$-vs. $18.1 \%$; $=13$-levels: $8.9 \%$-vs.-3.1\%; $\mathrm{p}=0.004)$. Overall operation time was $338.0 \pm 150.2$-minutes, HLOS 7.4 \pm 6.6 -days, $17.1 \%$ suffered 1 or more complications, and $54.5 \%$ were discharged home. On multivariable analysis, the combined approach $(\mathrm{B}=63.8$-minutes, 95\% CI [39.188.5], $\mathrm{p}<0.001$ ), and multilevel fusions (7-12: 61.0 [38.4-83.6]; $\mathrm{p}<0.001$; $=13: 133.8$ [98.0-169.6]; $\mathrm{p}<0.001$ ) associated with increased operation time. HLOS increased for multilevel fusions (7-12: 1.3-days [0.3-2.3]; $\mathrm{p}=0.012 ;=13: 2.2$ [0.6-3.8]; $\mathrm{p}=0.008)$. Overall complications did not differ by cohort or levels, however on post-hoc analysis the combined approach was associated with increased odds of pneumonia (OR $3.05[1.33-6.96] ; p=0.008)$. Multilevel fusions showed decreased odds of discharge to home $(7-12$ : $\mathrm{OR}=0.57$ [0.40-0.82]; $\mathrm{p}=0.003 ;=13$ : $\mathrm{OR}=0.41$ [0.22-0.73]; $\mathrm{p}=0.003)$.

Conclusion: The profile of 30-day outcomes is comparable for posterior vs. combined approaches in the elderly. Multilevel fusions increase operation time, HLOS, and discharge to higher level of care.

187. Early Ambulation Decreases Length of Hospital Stay, PeriOperative Complications and Improves Functional Outcomes in Elderly Patients Undergoing Surgery for Correction of Adult Degenerative Scoliosis

Owoicho Adogwa, MD, MPH; Aladine A. Elsamadicy, BE; Jared Fialkoff, BS; Joseph S. Cheng, MD, MS; Isaac O. Karikari, MD; Carlos Antonio Bagley, MD

Introduction: Prolonged immobilization after surgery can result in functional decline and an increased risk of hospital-associated complications. Whether early mobilization after elective spine surgery for correction of adult degenerative scoliosis improves functional outcomes, shortens length of stay, decreases the incidence of peri-operative complications, and 30-day hospital readmission rates remains unknown.

Methods: We conducted an ambispective study of 125 elderly patients ( $>65$ years old) undergoing elective spinal surgery for correction of adult degenerative scoliosis. Patient demographics, comorbidities, and post-operative complication rates were collected. We identified all unplanned readmissions within 30 days of discharge. "Days of immobility" was defined as the number of days until a patient moved out of bed beyond a chair. Patients in the top and bottom quartiles were dichotomized into "Early ambulators" and "Late ambulators", respectively. "Early ambulators" were ambulatory within 24 hours of surgery, while "Late ambulators" were ambulatory at a minimum of 48 hours following surgery. Complication rates, duration of hospital stay, and 30-day readmission rates were compared between cohorts.

Results: One hundred and twenty-five patients (Early ambulators: $\mathrm{n}=66$ patients vs. Late ambulators: $\mathrm{n}=59$ patients) were enrolled in this study. Baseline characteristics were similar between both cohorts. Compared to patients with a longer duration of immobility(i.e "Late ambulators"), the prevalence of at least one peri-operative complication was significantly lower in the "Early ambulators" cohort (30\% vs. $54 \%, p=0.06)$. The length of in-hospital stay was $34 \%$ shorter in the "Early Ambulator" cohort (5.33 days vs. 8.11 days, $p=0.01$ ). Functional independence was superior in the "Early ambulator" cohort, with the majority of patients discharged directly home after surgery compared to "Late ambulators" ( $71.2 \%$ vs. $22.0 \%, \mathrm{p}=0.01$ ).

Conclusion: In our experience, early ambulation after surgery significantly reduces the incidence of peri-operative complications, shortens duration of in-hospital stay, and contributes to improved peri-operative functional status in elderly patients undergoing surgery for correction of adult degenerative scoliosis. 
188. Use of the Adult Spinal Deformity (ASD) Frailty Index (ASD-FI) to Predict Major Complications in the Scoli-Risk 1 Multicenter, International Patient Database

Lawrence Lenke; Emily K. Miller; Daniel M. Sciubba, MD; Justin K. Scheer, MD; Brian J. Neuman, MD; Justin S. Smith. MD, PhD; Benny Dahl, MD, PhD, DMSci; Michael G. Fehlings, MD, PhD, FRCS (C), FACS; Kenneth Cheung, MBBS, MD; Leah Y. Carreon MD, MSc; Christopher I. Shaffrey MD, FACS; Khaled Kebaish, MD; Christopher P. Ames, MD; International Spine Study Group

Introduction: Patients with a greater degree of deformity, those with neuromuscular or congenital deformity, and those undergoing more complex procedures including 3 column osteotomies have an increased rate of major complications. In this complex cohort, accurate preoperative risk assessment is even more important. A deformity-specific frailty index (ASD-FI) has been validated in less complex cohorts and was strongly associated with major complication incidence and hospital length of stay (LOS).

Methods: The Scoli-Risk 1 database only included patients with severe deformity (eg $>80$ ? Cobb curvature or congenital deformities) undergoing complex procedures (eg 206/272 patients had 3-column osteotomies). The recently developed ASD-FI was used to calculate frailty scores for all patients. Patients were classified as not frail $(<0.3$ $\mathrm{NF})$, frail $(0.3-<0.5 \mathrm{~F})$, and severely frail $(=0.5 \mathrm{SF})$. We then performed a multivariate logistic regression to determine the relationship between ASD-FI cohorts, incidence of major complications, and hospital length of stay (LOS), adjusted for important preoperative and surgical covariates such as operative time and estimated blood loss.

Results: Of 267 participants, the mean ASD-FI score was 0.33 $(0-0.72)$. The odds of having a major complication was 1.5 [0.92.8] $(\mathrm{p}=0.1)$ for $\mathrm{F}(\mathrm{n}=103)$ and $3.6[1.8-7.0](\mathrm{p}<0.001)$ for $\mathrm{SF}(\mathrm{n}=59)$ compared to NF pts $(n=105)$. After adjusting for operative covariates, the odds ratios still trended upwards with frailty (1.5 for F, [0.8-2.8] $\mathrm{p}=0.3$ ) and 4.1 [1.9-9.2] $\mathrm{p}<0.001$ for SF). Unadjusted, LOS increased with frailty, increasing 4.0\% [-13.5-25.0] $(\mathrm{p}=0.6) \mathrm{F}$ and $11.9 \%$ [-9.838.9] $(p=0.3)$ SF from the LOS of NF pts. Adjusted, LOS increased $6.5 \%$ for $F$ [-6.9-21.7] $(\mathrm{p}=0.4)$ and $18.9 \%$ [1.4-39-4] $(\mathrm{p}<0.05)$ for $\mathrm{SF}$ compared to NF.

Conclusion: Frailty, as measured by the ASD-FI, was associated with increased risk of major complications and LOS (after controlling for site/region differences) for complicated ASD patients undergoing complex spine surgery. This validation of the index in the most complex deformity patients reinforces the role ASD-FI could play to improve the accuracy of preoperative risk stratification.

189. Despite Higher Risk Stratification Scores, Frail Patients Achieve Greater Two-year Health Related Quality of Life Improvement from Baseline Compared to Non-frail Patients Following Adult Spinal Deformity Surgery

Emily K Miller; Tamir T. Ailon, MD, MPH; Justin Kemp Scheer, MD; Alan H. Daniels, MD; Justin S. Smith, MD, PhD; Frank Schwab, MD, PhD; Virginie Lafage, PhD; Douglas C. Burton, MD; Robert Hart, MD; Shay Bess, MD; Christopher I. Shaffrey, MD, FACS; Christopher P. Ames, MD; International Spine Study Group

Introduction: The ASD-FI is a risk stratification tool which predicts major complications and prolonged hospital length of stay. The impact of frailty on postoperative improvement in HRQOL is unknown.

Methods: ASD patients who underwent $=4$ level instrumented fusion and had minimum 2-year follow-up were stratified by ASDFI score into categories: not frail 0-3 (NF); frail 3-5 (F); and severely frail $>5$ (SF). We compared baseline demographic, HRQOL, and radiographic parameters. The primary outcome measure was reaching substantial clinical benefit SCB (SCB) in ODI, SF-36 PCS, back and leg pain. Secondary outcomes included: absolute and change in ODI, PCS, MCS, back and leg pain. SCB thresholds for outcome following lumbar fusion were utilized (Table 1).

Results: 332 patients were identified with 2-year follow-up: 135 $\mathrm{NF}, 175 \mathrm{~F}$, and $22 \mathrm{SF}$. F and SF patients were significantly older, had more comorbidities, worse baseline HRQOL and pain scores (e.g. ODI 69.5, 52.5, 27.6 for SF,F, and NF; $\mathrm{p}<0.0001$ ), and worse radiographic deformity (e.g. SVA $130.5,85.9$, and $28.4 \mathrm{~mm}$ for SF, F, and NF; $p<0.0001$ ). At 2-years, ODI, PCS, MCS, back and leg pain were all worse in F/SF than NF patients. More NF than $\mathrm{F}$ patients reached SCB for back pain (63.4 vs. $57.5 \%$; $\mathrm{p}=0.045)$ whereas more $\mathrm{F}$ than NF reached SCB for ODI (43.7 vs. 29.3\%; $\mathrm{p}=0.025)$, PCS (56.9 vs. $51.2 \%$; $p=0.03$ ), and leg pain ( 45.8 vs. $23.0 \%$; $p=0.003)$. SF patients were least likely to achieve SCB for ODI (28.6\%), PCS (18.2\%), and back pain $(28.6 \%)$ (Table 1$)$.

Conclusion: Despite higher preoperative risk stratification scores, worse baseline HRQOL scores, and greater complication rates, frail patients experience greater improvement in HRQOL and likelihood of reaching substantial clinical benefit compared to non-frail patients.

190. Impact of Surgical Approach Translates on Complication Rates After Elective Spinal Fusion (e"3 Levels) for Adult Spine Deformity

Aladine A. Elsamadicy, BE; Owoicho Adogwa, MD, MPH; Shay Behrens, BS; Angel Chen, BS; Ankit Mehta, MD; Raul A. Vasquez, MD; Joseph S. Cheng, MD, MS; Carlos Antonio Bagley, MD; Isaac O. Karikari, $M D$

Introduction: While there are variations in techniques and surgical approaches to spinal fusion, there is not a defined consensus on a recommended surgical approach. The aim of this study is to determine if there was a difference in intra- and post-operative complication rates between different surgical approaches after elective spinal fusion (=3-levels) for adult spine deformity.

Methods: The medical records of 443 adult ( $=18$ years old) spine deformity patients undergoing elective spinal fusion(=3-levels) at a major academic institution from 2005 to 2015 were reviewed. We identified $96(21.7 \%)$ anterior only, 225(50.8\%) posterior only, and $122(27.5 \%)$ combined anterior/posterior approaches taken for spinal fusion (Anterior: $n=96$, Posterior: $n=225$ ). Patient demographics, comorbidities, anatomical location, intra- and post-operative complication rates were collected for each patient. The primary outcome investigated in this study was the rate of intra- and postoperative complications.

Results: Patient demographics and comorbidities were similar between all groups. The anatomical locations of the fusions were similar between all groups, with lumbar-only and thoraciclumbar fusions being most common. The posterior approach had significantly higher $\operatorname{EBL}(\mathrm{p}<0.0001)$ and number of PRBC blood transfusions $(p<0.002)$, while the combined approach had a higher operative time $(p<0.0001)$. The posterior approach had a significantly higher rate of intraoperative durotomies than anterior and combined (Anterior: 0\% vs. Posterior: $11.1 \%$ vs. Combined: $4.1 \% \mathrm{p}<0.0001$ ). Postoperative complications were similar between all the groups, with the combined cohort having a higher rate of delirium, and the posterior cohort having a higher rate of postoperative fever and patients being discharged with a Foley catheter. There was no significant difference in the rate 30 -day readmissions between the cohorts (Anterior: 10.4\% vs. Posterior: 12.8\% vs. Combined: $13.1 \%, \mathrm{p}=0.80)$.

Conclusion: Our study suggests that there may be a difference in surgical outcomes based on operative approach, with the posterior approach more susceptible to increased complications compared to the anterior or combined anterior/posterior approaches. 
191. Asymmetric Pedicle Subtraction Osteotomy for Adult Spinal Deformity with Coronal Imbalance: Complications, Radiographical and Surgical Outcomes

Andrew Kai-Hong Chan, MD; Darryl Lau, MD; Sigurd Berven, MD; Shane Burch; Serena Hu; Praveen V. Mummaneni, MD; Vedat Deviren, $M D$; Christopher P. Ames, $M D$

Introduction: Asymmetric pedicle subtraction osteotomy (APSO) with posterior spinal fusion can be utilized for adult spinal deformity (ASD) with fixed coronal plane imbalance. There are few reports investigating outcomes following APSO and no series that include multiple revision cases.

Methods: All thoracolumbar ASD cases from 2004-2016 at one institution were retrospectively reviewed. Pre-operative and latest follow up radiographical parameters and data on surgical outcomes and complications were obtained.

Results: There were 14 patients who underwent APSO with mean follow-up of 26.7 months. $10(71.4 \%)$ were revision cases. $3(21.4 \%)$ involved staged procedures. APSO surgeries involved a mean 12 levels (range 7-25) and were associated with $3.0 \mathrm{~L}$ of blood loss (range 1.2-4.5) and 457 minutes of operative time (range 283-540). Operations were associated with 1.9 days of ICU stay (range 0-6) and with 8.7 days of hospitalization (range 6-15). $42.9 \%$ of patients were discharged to home or home health care. Surgical complications were observed in $57.1 \%$ of patients comprising durotomy $(35.7 \%)$, pleural injury (14.3\%), persistent neurologic deficit (14.3\%), and painful iliac bolt requiring removal $(7 \%)$. Medical complications were observed in $14.3 \%$ of patients comprising urosepsis and 2 cases of pneumonia. There were two 90-day readmissions $(14.3 \%)$ for pneumonia and for revision osteotomy after neurologic deficit. There were 4 re-operations ( 3 patients, 21.4\%) for a painful iliac bolt, L5 radiculopathy, and pseudoarthrosis (2 re-operations). Mean lumbar scoliosis and coronal vertical axis improved from 27.1 to 13.6 degrees and 8.1 to $2.8 \mathrm{~cm}$, respectively. PI-LL mismatch, mean sagittal vertical axis, and pelvic tilt improved from 38.0 to 16.1 degrees, 10.9 to $4.5 \mathrm{~cm}$, and 33.6 to 26.1 degrees, respectively.

Conclusion: The APSO, in both a revision and non-revision population, has an acceptable complication profile and provides excellent restoration of coronal balance, in addition to sagittal and pelvic parameters, in adults with spinal deformity.

192. Adult Spinal Deformity Patients with Previous Fusions Have an Equal Chance of Reaching Substantial Clinical Benefit Thresholds in Health-related Quality of Life Measures but Do Not Reach the Same Absolute Level of Improvement

Tamir T. Ailon, MD, MPH; Justin S. Smith, MD, PhD; Christopher I. Shaffrey, MD, FACS; Alex Soroceanu; Virginie Lafage, PhD; Frank Schwab, MD, PhD; Douglas C. Burton, MD; Robert Hart, MD; Han Jo Kim, MD; Jeffrey Lynn Gum, MD; Richard A. Hostin, MD; Michael P. Kelly, MD; Steven D. Glassman; Justin K. Scheer, MD; Shay Bess, MD; Christopher P. Ames, MD; International Spine Study Group

Introduction: Substantial clinical benefit (SCB) represents a threshold above which a patient recognizes substantial benefit and may therefore be a desirable target outcome. We investigated the impact of prior spinal fusion on the likelihood of reaching SCB thresholds for 2-year health-related quality of life (HRQOL) after adult spinal deformity (ASD) surgery.

Methods: We included ASD patients who achieved minimum 2-year follow-up. We compared baseline demographic, HRQOL, and radiographic features for patients undergoing primary versus revision procedures. The primary outcome measure was reaching SCB threshold in Oswestry Disability Index (ODI), SF-36 physical component summary (PCS), back and leg pain (numeric rating scale;
NRS). Secondary outcomes included absolute and change scores in ODI, PCS, back and leg pain. We used SCB thresholds previously reported for outcome following lumbar fusion.

Results: 332 patients achieved 2-year follow-up, including 228 primary and 104 revision cases. Those undergoing revision surgery had similar demographic features (age 58.3/55.9, female $80.8 / 82.9 \%$, Charlson Comorbidity Index (1.9/1.6) to primary surgery patients. Revision surgery patients had worse baseline HRQOL (ODI 48.5/41.2, PCS 29.5/33.4, back 7.5/7.0, and leg pain 4.9/4.3; $<<0.001$ ) and radiographic deformity (sagittal vertical axis 111.4/45.1, pelvic incidence to lumbar lordosis mismatch 26.7/11.0, pelvic tilt 29.5/21.0; $\mathrm{p}<0.0001)$. Nevertheless, the number of patients who reached SCB for ODI (38.3/36.3\%), PCS (48.5/53.4\%), back (53.1/60.5\%) and leg pain NRS (28.6/36.9\%) did not significantly differ between revision and primary operations. Despite similar magnitudes of improvement of outcomes measures, revision patients had worse 2-year HRQOL for all measures compared with primary surgery patients.

Conclusion: Patients undergoing revision ASD surgery have worse baseline HRQOL and radiographic deformity. Although they do not achieve the same absolute level of 2-year HRQOL outcome scores as patients undergoing primary surgeries, they have a similar likelihood of reaching SCB threshold for improvement in 2-year HRQOL.

193. Under-Correction of Sagittal Deformities Based on AgeAdjusted Alignment Thresholds Leads to Worse HRQOL While Over-Correction Provides No Additional Benefit

Justin Kemp Scheer, MD; Renaud Lafage; Barthelemy Liabaud; Frank Schwab, MD, PhD; Justin S. Smith, MD, PhD; Gregory M. Mundis; Richard A. Hostin, MD; Christopher I. Shaffrey, MD, FACS; Douglas C. Burton, MD; Han Jo Kim, MD; Shay Bess, MD; Munish C. Gupta, MD; Virginie Lafage, PhD; Christopher P. Ames, MD

Introduction: Recent research in sagittal plane proposed ageadjusted alignment thresholds. However, impact of these thresholds on post-operative HRQL is yet to be investigated. This study aims to compare $2 \mathrm{yr}$ clinical outcomes of patients (pts) who underwent surgical reconstructions based on their achievement to age-adjusted alignment ideals.

Methods: Retrospective review of prospectively collected database. Patients were included if $>18 \mathrm{y} / \mathrm{o}$, underwent surgical correction of adult spinal deformity with a complete $2 \mathrm{yr}$ FU. Pts were stratified into 3 groups based on achievement of age-adjusted thresholds in pelvic tilt (PT), pelvic incidence minus lumbar lordosis (PI-LL), sagittal vertical axis (SVA). First group included pts who reached the exact age-adjusted threshold \pm 10 yrs (MATCHED), other two groups included pts who were over corrected (OVER), and under corrected (UNDER). Clinical outcomes including actual value and offset from age-adjusted ODI, SF36-PCS and SRS-22 (PROM) were compared between groups at $2 \mathrm{yr} \mathrm{FU}$

Results: 343 patients (57.0yo and $83 \%$ F) were included. Sagittal profile of the population was: $\mathrm{PT}=23.6^{\circ}, \mathrm{SVA}=65.8 \mathrm{~mm}, \mathrm{PI}-\mathrm{LL}=15.6^{\circ}$. At 2-year follow-up there was a significant improvement in all sagittal modifiers with $25.7 \%, 24.3 \%$ and $33.1 \%$ of the patients matching their age alignment targets in terms of PT, PI-LL and SVA respectively. For PT and PI-LL the 3 groups (MATCHED, OVER, UNDER) had comparable values and offsets from age-adjusted PROM. However, for SVA groups, patients in UNDER had significantly worse HRQOL than the two other groups. Patients in PT, PI-LL, and SVA UNDER groups were significantly younger than the other groups, $\mathrm{p}<0.05$.

Conclusion: At $2 \mathrm{yr}$ following ASD surgical treatment only $24.3 \%$ to $33.1 \%$ of the pts reached age-adjusted alignment thresholds. Those under corrected in SVA demonstrated worse clinical outcomes. No significant improvements were found between matched and overcorrected pts, with overcorrection being an established risk for PJK. These results further emphasize the need for patient specific operative planning. 
194. Use of a Validated Predictive Model For Patient Selection in Adult Spinal Deformity (ASD) Surgery Has the Potential to Enhance QALYs Gained at 2-years: Simulation in 234 ASD Patients

Richard A. Hostin, MD; Justin Kemp Scheer, MD; Chessie Robinson, MA; Jeffrey Lynn Gum, MD; Frank Schwab, MD, PhD; Robert Hart, MD; Virginie Lafage, PhD; Douglas C. Burton, MD; Shay Bess, MD; Themistocles Protopsaltis, MD; Eric Klineberg, MD; Christopher I. Shaffrey, MD, FACS; Justin S. Smith, MD, PhD; Christopher P. Ames, $M D$

Introduction: A preop predictive model for meeting 2yr ODI MCID may be useful to determine health utility improvement and thus could aid in selection for cost effective care. Also, it is unknown if an ODI MCID model can identify patients with QALY improvements. The goal of this study was to build a preop predictive model for meeting 2yr ODI MCID and use it to relate QALYs gained at $2 \mathrm{yrs}$.

Methods: Retrospective review of prospective multicenter ASD database. Inclusion criteria: age $=18$, ASD, preop, 1yr, $2 \mathrm{yr}$ followup, preop ODI $>15$. 46 baseline variables were used to train the model: demographics, comorbidities, HRQOL, coronal and sagittal radiographic values, and modifiable surgical variables. Pts grouped by reaching $2 \mathrm{yr}$ ODI MCID or not. An ensemble of 5 different boostrapped C5.0 algorithm decision trees was constructed. Internal validation included 70:30 data split for training and testing each model, respectively. Accuracy and area under a receiver operator characteristic curve (AUC) were calculated. SF6D scores used to calculate QALYs gained at 2yrs and mean 2yr QALYs which were discounted at the recommended 3.5\%/yr. QALYs were compared between pts predicted to meet MCID and those not.

Results: 234 patients were included, (+MCID:129, -MCID:105). The testing data consisted of 69(29.5\%) pts and of those, the predicted versus actual results were: +MCID:50vs.40,-MCID:19vs.29; resulting in 10 misclassified pts with an accuracy of $85.5 \%$ and 0.96 AUC. From the predicted results of the testing dataset, the +MCID pts had significantly greater $2 \mathrm{yr}$ mean QALYs gained $(0.232 \pm 0.22$ vs. $-0.016 \pm 0.19, p=0.0002$, Figure)

Conclusion: Based on a successful 2yr ODI MCID model, $(85.5 \%$ accuracy, 0.96 AUC) those predicted to meet MCID had significantly improved 2yr QALYs gained despite 10 of the pts being misclassified. This provides a proof-of-concept for predictive modeling in $\mathrm{pt}$ selection and QALY estimation and sets the groundwork for applied predictive analytics in spine outcomes research.

195. Predictive Modeling of Length of Hospital Stay (LOS) Following Adult Spinal Deformity (ASD) Correction: Analysis of 653 Patients with an Accuracy of 75\% Within 2 Days

Justin Kemp Scheer, MD; Tamir T. Ailon, MD, MPH; Justin S. Smith, MD, PhD; Robert Hart, MD; Douglas C. Burton, MD; Shay Bess, MD; Brian J. Neuman, MD; Peter G. Passias, MD; Emily Miller; Christopher I. Shaffrey, MD, FACS; Frank Schwab, MD, PhD, Virginie Lafage, PhD; Eric Klineberg, MD; Christopher P. Ames, $M D$

Introduction: The LOS following ASD surgery is a critical time period allowing for recovery to levels safe enough to return home or to rehabilitation. Thus, the goal is to minimize it for conserving hospital resources and third party payer pressure. Factors related to LOS have not been studied nor has a predictive model been created. The goal of this study was to construct a preadmission predictive model based on patients' (pts) baseline variables and modifiable surgical parameters.

Methods: Retrospective review of a multicenter, prospective ASD database. Inclusion criteria: operative pts, age $>18 y$ rs, ASD.
Pts with staged surgery at a separate hospitalization or LOS $>30$ days were excluded. 66 variables were initially evaluated with 40 being used for model building following univariable predictor importance $=0.90$, redundancy, and collinearity testing. Variables included: demographics, comorbidities, preop HRQOL, preop coronal and sagittal radiographic parameters, and modifiable surgical factors (Figure). A generalized linear model was constructed using a training dataset developed from a boostrapped sample with replacement using a random number generator. Pts randomly omitted from the boostrapped sample composed the testing dataset. Accuracy was calculated by comparison of predicted LOS to the actual LOS.

Results: A total of 689 patients were eligible with 653 meeting inclusion criteria. The mean LOS was $7.9 \pm 4.1$ days (range: 1-28). Following bootstrapping, 893 pts were modeled in total, Training:653, Testing:240(36.6\%). The linear correlations for the training and testing datasets were 0.632 and 0.507 , respectively. Testing dataset accuracy within 2 days of actual LOS was 75.4\% (181/240 pts). Predictor importance rankings are listed in the Figure.

Conclusion: A successful model was created to predict LOS to an accuracy of $75 \%$ within 2 days. There are some factors related to LOS that are not likely captured in large databases, which may partially explain the $75 \%$ accuracy, such as rehab bed availability and social support resources.

196. The Impact of Different Intraoperative Fluid Administration Strategies on Postoperative Extubation Following Multi-Level Thoracic and Lumbar Spine Surgery: A Propensity Score Matched Analysis

Subaraman Ramchandran, MBBS, MS; Shay Bess, MD; Louis Day, BS; Breton G. Line, BSME; Aaron James Buckland, MBBS, FRACS; Themistocles Protopsaltis, MD; Peter G Passias, MD; Thomas Errico

Introduction: Immediate extubation following surgical procedures reduces complications and shortens hospital stay. Patients undergoing multi-level spine surgery are at risk for delayed extubation. Study purpose: To evaluate the impact that the type and volume of intraoperative fluids administered during multi-level thoracic and/or lumbar spine surgery has upon postoperative extubation status.

Methods: Medical records of patients $=18$ years undergoing $=4$ levels of thoracic and/or lumbar spine fusion procedures were evaluated. Patients were organized according to postoperative extubation status: immediate (IMEX; in OR/PACU) or delayed (DEX; outside OR/PACU). Patients were divided according to intraoperative estimated blood volume loss (EBVL); $<15 \%, 15-30 \%$, $>30 \%$ of EBVL. Propensity score matched (PSM) analysis was performed to compare IMEX and DEX groups. Volume, proportion and ratios of intra-operative fluids administered were evaluated for the associated impact on extubation status.

Results: 246 patients (198 IMEX, 48 DEX) were included. PSM analysis demonstrated that increased administration of non-cell saver blood products (NCSB) and increased ratio of crystalloid colloids infused were independently associated with delayed extubation. Increased EBL was associated with increased NCSB infusion for IMEX and DEX, however, with increasing EBL, IMEX had a proportionate reduction in crystalloid infusion $(\mathrm{R}=-0.5, \mathrm{p}<0.001)$, while the proportion of crystalloids infused remained relatively unchanged for $\operatorname{DEX}(\mathrm{R}=-0.27 ; \mathrm{p}=0.06)$. Twenty-six percent of patients receiving crystalloid: colloid ratio $>3: 1$ had delayed extubation compared to none of those receiving crystalloid: colloid ratio $=3: 1$ $(p=0.009)$. DEX had greater cardiac and pulmonary complications, surgical site infections and prolonged ICU and hospital stay $(\mathrm{p}<0.05)$.

Conclusion: PSM analysis of patients undergoing multi-level thoracic and/or lumbar spine fusion demonstrated that increased administration of crystalloid to colloid ratio is independently associated with delayed extubation. With increasing EBL, a 
proportionate reduction of crystalloids facilitates early extubation. Administration of crystalloid: colloid ratio $>3: 1$ increases the risk for delayed extubation. Delayed extubation is associated with increased in-hospital complications and prolonged hospital stay.

\section{Direct Costs of Adult Scoliosis in the United States}

Rory R. Mayer, MD; Terence Verla, MD, MPH; I-Wen Pan, PhD; Ben Allen Strickland, MD; Andrew Jea, MD; Sandi Lam, MD, MBA

Introduction: In this current legislative era, it has become paramount to define the costs of surgical care to help guide payers and reimbursement models. We sought to develop estimates of the direct costs in the treatment of adult scoliosis in the U.S. by systematic evaluation of published studies and to compare similarities and differences in types of estimates, methods, and cost parameters.

Methods: Papers were identified by systematic 3-stage literature search including estimates of average annual direct costs for the treatment of adult scoliosis in the U.S. Type of scoliotic deformity, study design, estimation method, and data sources were extracted. Annual cost estimates were identified and compared.

Results: 9 of 1549 publications discovered met criteria for review. For patients with adult scoliosis, (comprising all subtypes including idiopathic, degenerative, sagittal plane deformity and revision), direct nonoperative costs per person ranged from $\$ 4,852-5558$ per year and hospitalization costs with deformity correction ranged from $\$ 75,410$ to $\$ 391,889$. Direct surgical costs ranged from $\$ 46,051-\$ 72,034$, with costs on readmission between $\$ 46,599$ and $\$ 75,707$. Despite adjustment to 2015 dollars, the data suggests significant increases in the cost of overall hospitalization in the past fifteen years.

Conclusion: This analysis is the first to appraise the literature in a systematic fashion in an attempt to define direct costs associated with the treatment of adult scoliosis in the United States. We sought to identify the methodological differences between studies, including an evaluation of their respective data sources and methods for cost estimation. Our analysis suggests that the conservative treatment of adult scoliosis carries a high cost and resource utilization burden. Although the costs of index surgery are high, revision surgery and rehospitalization have a significant impact on total direct costs. Our study demonstrates that the vast majority of cost papers evaluating adult scoliosis are limited to single or limited multi-center samples.

198. Biomechanical Study to Determine Change in Range-ofMotion of Human Thoracic Cadaveric Spine with Intact Rib Cage Following Sequential Ponte Osteotomies

Erin M. Mannen, MS; Paul M. Arnold, MD; John T. Anderson, MD; E.(Lisa) A. Friis, PhD

Introduction: Adolescent idiopathic scoliosis and Scheuermann's kyphosis are spinal deformities affecting the lateral and sagittal planes; posterior Ponte osteotomies (POs) are often performed to provide flexibility in the sagittal plane at an estimated correction potential of $5^{\circ}$ per PO. POs remove the posterior spinous process, the ligamentum flavum, and the facets at the apex of the sagittal curve. No studies have quantified the change in range-of-motion (ROM) of the human cadaveric thoracic spine with intact rib cage following sequential POs.

Methods: Seven fresh-frozen male human cadaveric thoracic specimens (T1-T12) with intact rib cages were dissected to include stabilizing ligaments, vertebrae, intervertebral discs, and ribs. Specimens were thawed, potted parallel to the T1 and T12 endplates, and mounted in a six-degree-of-freedom spine testing machine (Applied Test Systems, Butler, PA). Motion capture pins were inserted into the right pedicles of T6-T10 and at the top potting, representing
T1. Pure moments were applied to T1 in flexion-extension, lateral bending, and axial rotation at a rate of $1 \%$ second to a load limit of $5 \mathrm{~N} \cdot \mathrm{m}$ for five conditions: intact, $\mathrm{PO}$ at T9-T10, PO at T8-T9, $\mathrm{PO}$ at T7-T8, and PO at T6-T7. Motion of T1, T6, and T10 were measured, and overall (T1-T12) and regional (T6-T10) ROMs were calculated for each mode of bending at each condition.

Results: POs increased ROM in flexion only, both overall (T1T12) and regionally (T6-T10), although the magnitude of the increase was marginal $(<1 \%$ PO). No significant differences were found in axial rotation or lateral bending.

Conclusion: POs may increase sagittal correction potential before fusion in patients with hyperkyphosis. More in vitro work should be done to determine the magnitude of the changes, and to examine if the level at which the PO is performed affects correction potential, which could inform surgeons of the most appropriate levels for POs.

\section{Neurologic and Radiographic Outcomes of Anterior Column Realignment (ACR)}

Rajiv Saigal, MD, PhD; Gregory M. Mundis; Robert Eastlack, MD; Stacie Nguyen, BS, MPH; Behrooz A. Akbarnia, MD

Introduction: Anterior Column Realignment (ACR) is a less invasive alternative to three column osteotomy for correction of sagittal imbalance. ACR involves sectioning the anterior longitudinal ligament and placing a hyperlordotic cage via a lateral transpsoas approach. We aim to report a detailed analysis of neurologic risk and radiographic outcome after ACR.

Methods: Patients $>18$ who underwent ACR from 2005-2013 were eligible. Standing scoliosis radiographs were studied at pre-, and post op ( $<6$ weeks; EARLY) and latest follow up (LFU). Clinical/ radiographic data was collected by retrospective chart review, with T1SPI used as the angular surrogate for SVA. Wilcoxon signed rank and McNemar's test were used to assess significant differences.

Results: 35 patients met inclusion, 29 had complete data, with mean follow up 2.4 years (1-7). Pre-op, sagittal parameters were: LL of $-18.3^{\circ}$, PI-LL $40^{\circ}$, T1SPi $3.7^{\circ}$ and PT $32^{\circ}$. LL improved by $28.9^{\circ}$ $(\mathrm{p}<0.001)$. Mean change in PT $(-8)$, SS (7.1), T1SPi (-5.2), and PI-LL $(-29.9)$ were all significant (Table 1). The motion segment angle (superior endplate of vertebra above and inferior endplate of vertebra below) improved by $23.4^{\circ}$, from $3.8^{\circ}$ to $-19.3^{\circ}$ ( $\left.p<0.001\right)$. Neurologic complications occurred in $38 \%(n=11 ; 1$ patient with both sensory and motor). Thigh numbness/paresthesia in $5(17 \% ; \mathrm{p}=0.063)$ at 6 weeks; all resolved at LFU. 9 (31\%) had pre-op motor deficit and 7 (24.1\%) developed a new deficit $(\mathrm{p}=0.549)$, with 3 (10.3\%) having persistent weakness at LFU. Of the original 9 with pre-op weakness, 3 had persistent weakness. Patients saw improvement in VAS, ODI and SRS $(\mathrm{p}<0.05)$.

Conclusion: There was net neurologic improvement, with $33 \%$ improvement in pre-op deficits and $11 \%$ persistent new weakness at latest follow up. Radiographic results demonstrate that ACR is a useful tool to address patients with severe sagittal plane deformity with improvement in HRQOL despite its inherent neurologic risk.

200. Neurological Comorbidities Predict Proximal Junctional Kyphosis: A Case-Matched Cohort Analysis Performed at a Single Center

Hideyuki Arima, MD, PhD; Steven D. Glassman; John R. Dimar, $M D$; Leah Y. Carreon, $M D, M S c$

Introduction: Proximal Junctional Kyphosis (PJK) is a common and potentially devastating complication following surgery for adult spinal deformity (ASD). Although proposed prevention strategies include restoration of spinal balance, cement augmentation, and use 
of softer transitions such as hooks, the effectiveness of these measures has been limited. Recently, we reported that non-mechanical neurological comorbidities play an important role in postoperative sagittal imbalance and PJK. This study was performed to further define the contribution of non-mechanical factors to the occurrence of PJK after ASD.

Methods: We identified a consecutive series of ASD patients who required revision surgery for PJK between 2013 and 2015. A matched cohort of ASD patients that did not develop PJK was identified based upon age, gender, preoperative deformity type and number of fusion levels. We compared medical and surgical histories in the matched cohorts, with particular attention to the prevalence of preoperative neurologic comorbidities that might affect standing balance. Preoperative, immediate postoperative and followup radiographs were reviewed to document specific characteristics of mechanical failure that resulted in PJK and required revision surgery.

Results: Twenty-eight cases of PJK requiring revision surgery were identified. The prevalence of pre-operative neurological comorbidities in PJK patients were statistically significantly higher than in non-PJK patients $(75 \%$ vs. $32 \%, \mathrm{p}<0.001)$. Neurological comorbidities included prior stroke (4), metabolic encephalopathy (2), Parkinson's disease (1), seizure disorder (1), cervical and thoracic myelopathy (7), diabetic neuropathy (4) and other neuropathy (4). The mean preoperative sagittal vertical axis in PJK patients was more positive compared to non-PJK patients ( $143 \mathrm{~mm}$ vs. $65 \mathrm{~mm}, \mathrm{p}=0.009$ ). There were no significant differences in immediate postoperative or follow-up radiographic parameters between cohorts.

Conclusion: Risk factors identified for the development of PJK included non-mechanical neurological comorbidities, emphasizing the need to look beyond radiographic alignment in order to reduce the incidence of PJK.

\section{Bimodal Incidence and Causes of Proximal Junctional Kyphosis (PJK) in Adult Spinal Deformity (ASD)}

Munish C. Gupta, MD; Bassel G. Diebo, MD; Themistocles Protopsaltis, MD; Robert Hart, MD; Justin S. Smith, MD, PhD, Christopher P. Ames, MD; Renaud Lafage; Justin K. Scheer, MD; Han Jo Kim, MD; Shay Bess, MD; Douglas C. Burton, MD; Peter G. Passias, MD; Frank Schwab, MD, PhD; Virginie Lafage, PhD; International Spine Study Group

Introduction: PJK is a well-known complication occurring in patients (pts) that undergo surgical ASD correction. Acute PJK (aPJK) occurs within the first six weeks, and chronic PJK (cPJK) more than 1 yr after surgery. Both PJK deformities can require surgical treatment. In this study, we aimed to first report the incidence of PJK, and contributing factors to either acute or chronic PJK.

Methods: Pts were included if they were $>18 \mathrm{y},=5$ levels fused including the pelvis and $2 \mathrm{y} \mathrm{f} / \mathrm{u}$. Using Glattes criteria, pts were stratified according to timing of PJK: aPJK $<6 \mathrm{wk}$, and cPJK $>1 \mathrm{y}$. Pts demographics, radiographic parameters, clinical outcomes (HRQL), operative data, complications and revisions rates were studied using univariate and multivariate analyses to identify independent predictors of aPJK.

Results: 176 pts (61.8 yo) were included. 71 pts (40.3\%) developed aPJK, and 39 pts $(22 \%)$ cPJK. Both groups were similar in age, baseline deformity, and HRQL. aPJK pts were more likely to have Charlson score $>2$ (39.4\% vs. $20.5 \%$ ), depression (34\% vs. $16.2 \%$ ), greater \# of co-morbidities (2.7 vs. 2.0), all $\mathrm{p}<0.05$. aPJK pts were more likely to have an abnormal neurologic exam ( $30 \%$ vs. $10.5 \%)$, and unable to perform toe-walking test $(17 \%$ vs. $0 \%)$, all $\mathrm{p}<0.05$. aPJK had higher revision rate (21 vs. $10.3 \%$ ), peri-operative complication rate $(14.1 \%$ vs. $0 \%)$ all $p<0.05$. Both groups underwent similar amounts of sagittal correction, \# of level fused, \#/type of osteotomies, however, aPJK had more proximal LL apex restoration vs more caudal apex restoration in cPJK. aPJK occurred in 50/70 (71.4\%) of posterior only approaches vs. $21 / 40(52.5 \%)$ in combined approaches, $\mathrm{p}<0.05$. On multivariate analysis depression (OR: 2.99 ), and abnormal neurologic exam (OR: 4.15) increased the likelihood of aPJK.

Conclusion: Compared to chronic PJK, Acute PJK pts have more co-morbidities, neurologic deficits, depression, and higher lumbar apex corrections with less caudal correction. In addition, aPJK pts had significantly higher peri-operative complication rates, and were more likely to be revised.

202. Towards a Better Understanding of Cervical Sagittal Alignment: Utilizing a Novel Angle to Describe the Relationship between T1 Vertebral Body Slope, Cervical Lordosis, and Cervical Sagittal Alignment

Ezequiel Goldschmidt, MD, PhD; Federico Angriman; Marcos Trevisan, PhD; James Zhou, BS; Nitin Agarwal, MD; Katherine Chen, BS; Adam S. Kanter, MD; David O. Okonkwo, MD, PhD; Peter G. Passias, MD; Justin Kemp Scheer, MD; Themistocles Protopsaltis, MD; Virginie Lafage, PhD; Renaud Lafage; Frank Schwab, MD, PhD; Shay Bess, MD; Christopher P. Ames, MD; Justin S. Smith, MD, PhD; Douglas C. Burton, MD; D. Kojo Hamilton, MD; International Spine Study Group (ISSG)

Introduction: Cervical alignment has become increasingly important in the planning of spine surgery. A relationship between the slope of T1 (T1S), the cervical lordosis (CL), and the overall cervical sagittal vertical axis (cSVA) has been demonstrated, but the exact nature of this relationship is poorly understood. In this study theoretical and empirical equations were derived in order to better understand how T1S and CL affect cSVA.

Methods: Two equations were derived to elucidate the relationship between T1S, CL, and cSVA. The first equation was developed using a Pythagorean theorem-based examination of the cervical spine. By treating the cervical spine as the arc of a circumference and by taking into account the cervical height $(\mathrm{CH})$, the geometric relationship between the T1S, CL, and cSVA was described via a trigonometric identity utilizing a novel angle $\mathrm{d}$ subtended by the $\mathrm{CH}$ and cSVA ( $d=$ T1S-CL/2). The second equation was developed on an empiric basis by performing a multiple linear regression analysis on a large multicenter deformity database collected by the International Spine Study Group. The two equations were compared to determine if an underlying relationship could be identified.

Results: The theoretical equation determined that the value of cSVA can be expressed as: $c S V A=\mathrm{CH}^{*} \tan (\mathrm{p} / 180 *(\mathrm{~T} 1 \mathrm{~S}-\mathrm{CL} / 2)$. The empirical equation determined that value of cSVA can be expressed as: cSVA $=\left(1.1^{*} \mathrm{~T} 1 \mathrm{~S}\right)\left(0.43^{*} \mathrm{CL}\right)+6.69$. In both, the sagittal alignment of the head over the shoulders is directly proportional to the T1S and inversely proportional to $\mathrm{CL} / 2$.

Conclusion: Two simple equations were derived using different methods that provide insight into the relationship between cSVA, CL, and T1S. These equations may allow surgeons to better understand how the CL compensates for the T1S, to accurately predict the postoperative cSVA, and to customize cervical interbody grafts by taking into consideration each individual patient's cervical parameters.

203. Gliding Anterior Cervical Cage Placement Combined with Posterior Fusion for Correction of Fixed Cervical Deformity with Myelopathy

Andrew James Grossbach, MD; Oliver Flouty; Stephanus Viljoen, $M D$

Introduction: Cervical myelopathy in the setting of fixed cervical kyphosis poses a complex surgical problem 3 since facet ankylosis 
often requires a posterior-anterior-posterior approach to first release the facets1, then place anterior hardware, followed by posterior instrumentation2. The placement of standard anterior instrumentation as the initial step is made difficult by the fused facets posteriorly. Furthermore, placing an anterior plate prior to posterior release will lock the spine in suboptimal position. In our series, we explore a new technique whereby anterior integrated cages are placed with a single screw traversing the cranial or caudal vertebrae. This allows the cages to glide with subsequent posterior correction, minimizing the number of patient flips from four to two.

Methods: A retrospective review of patients was performed to identify patients treated with anterior interbody cages with integrated screws followed by a posterior instrumentation and fusion from July 2014 December 2015. Five patients were identified. C1-C7 lordosis, C2-7 lordosis, C2 sagittal vertical axis (SVA), and T1 slope were measured4 pre- and postoperatively.

Results: Average pre-operative measurements showed C1-7 lordosis $19.4^{\circ}(-14-53)$, C2-7 lordosis $-18.2^{\circ}(-61-10)$, SVA $6.0 \mathrm{~cm}(1.5-$ $10.4)$, and $\mathrm{T} 1$ slope $27.4^{\circ}(0-48)$ were done in five patients. Average follow-up was 9 months (3-15). Average post-operative imaging showed C1-7 lordosis $40.2^{\circ}(20-51)$, C2-7 lordosis $2.2^{\circ}(-510)$, SVA $5.6 \mathrm{~cm}(4-8.7)$, and $\mathrm{T} 1$ slope $34.8^{\circ}(31-41)$. There was a trend towards improved lordosis and decreased SVA. These values did not reach significance $(\mathrm{p}>0.05)$, likely due to the small number of patients. $80 \%$ reported symptomatic improvement.

Conclusion: We conclude that the use of integrated anterior interbody cages with a single screw placed into the cranial or caudal vertebral body followed by posterior facet release, and fusion is a powerful tool to address fixed cervical kyphosis while minimizing the number of patient flips from a total of four to two when compared to the back-front-back approach.

204. Primary Drivers of Cervical Deformity: Prevalence and Effect of Surgical Treatment Strategies on Post-Operative Alignment

Peter G Passias, MD; Cyrus M. Jalai, BA; Virginie Lafage, PhD; Renaud Lafage; Themistocles Protopsaltis, MD; Gregory $W$. Poorman; Subaraman Ramchandran, MBBS, MS; Samantha $R$. Horn; Munish C. Gupta, MD; Robert Hart, MD; Vedat Deviren, MD; Alex Soroceanu; Justin S. Smith, MD, PhD; Frank Schwab, MD, PhD; Christopher I. Shaffrey, MD, FACS; Christopher P. Ames, MD; International Spine Study Group (ISSG)

Introduction: Cervical spine deformity (CSD) primary drivers (PD) have yet to be evaluated in relation to pre/postop alignment. Ideal cervical deformity correction may be qualifiable by using the primary deformity driver. This study defines CSD drivers to understand the impact of driver region on postop sagittal realignment.

Methods: CSD patients (pts) $=18$ yrs with pre-/post-op (3M) radiographs. $\mathrm{PD}$ were classified based on spinal region: $\mathrm{CS}=$ cervical; $\mathrm{CTJ}=$ cervicothoracic junction; $\mathrm{TH}=$ thoracic; $\mathrm{SP}=$ spino-pelvic. Pts were evaluated if the surgery included the PD apex, based on lowest instrumented vertebra (LIV): $\mathrm{CS}$ : $\mathrm{LIV}=\mathrm{C} 7, \mathrm{CTJ}$ : $\mathrm{LIV}=\mathrm{T} 3, \mathrm{TH}$ : LIV=T12. Cervical alignment modifiers (cSVA, CBVA, mJOA, TS-CL) quantified post-op goals. Groups were compared with ANOVA/Pearson, and paired t-tests.

Results: 84 pts met criteria (mean age $63.1 y r s, 62 \% \mathrm{~F}$ ). Thoracic drivers $(n=26)$ showed the greatest pre-op cervical and global malalignment against other PD: higher TK, PI-LL, T1 Slope C2-T3 SVA, and C0-2 angle $(\mathrm{p}<0.05)$. Differences in baseline-3M alignment changes were observed between surgical PD groups, in PI-LL, LL, TS-CL, cSVA, C2-T3 SVA ( $p<0.05$ all cases). Main changes were between TH and CS driver groups: TH pts had greater PI-LL $\left(4.47^{\circ}\right.$ vs. $\left.-0.87^{\circ}, \mathrm{p}=0.049\right)$, TS-CL $\left(-19.12^{\circ}\right.$ vs. $\left.-4.30, \mathrm{p}=0.050\right), \mathrm{C} 2-7 \mathrm{SVA}$ (-18.12 vs. $-4.30 \mathrm{~mm}, \mathrm{p}=0.007)$, and C2-T3 SVA (-24.76 vs. $8.50 \mathrm{~mm}$, $\mathrm{p}=0.002)$ baseline-3M correction. CTJ drivers also had a trend toward greater LL correction compared to CS drivers $\left(-6.00^{\circ}\right.$ vs. $0.88^{\circ}$, $\mathrm{p}=0.050)$. In pts operated at CS driver level, there was a significant difference in the prevalence of $3 \mathrm{M}$ TS-CL modifier grades $(0=35.7 \%$, $1=0.0 \%, 2=13.3 \%, p=0.030$ ). There was also a significant difference in the 3M CBVA modifier grade distribution in pts operated at the $\mathrm{TH}$ driver level $(0=0.0 \%, 1=35.9 \%, 2=14.3 \%, p=0.049)$.

Conclusion: Characterizing CSD patients by primary driver type reveals differences in pre- and post-operative alignment. Evaluating surgical alignment outcomes based on PD inclusion is important in understanding alignment goals for CSD correction.

205. Reciprocal Changes in Unfused Spinal Regions After Treatment of Cervical Deformity Based on Deformity Driver and Surgical Strategies

Peter G. Passias, MD; Cyrus M. Jalai, BA; Renaud Lafage; Subaraman Ramchandran, MBBS, MS, (orth); Gregory W, Poorman; Virginie Lafage, PhD; Justin S. Smith, MD, PhD; Samantha R. Horn; Vedat Deviren, MD; Robert Hart, MD; Themistocles Protopsaltis, MD; Alan H. Daniels, MD; Eric Klineberg, MD; Christopher I. Shaffrey, MD, FACS; Christopher P. Ames, MD; International Spine Study Group (ISSG)

Introduction: Reciprocal changes (RC) may occur if the primary driver (PD) of cervical spine deformity (CSD) is included in the fusion construct. RC in spinal alignment adjacent to fusions for CSD correction are poorly understood, notably in relation to surgical strategy for deformity driver location.

Methods: CSD patients=18yr with pre-/post-op (3M) radiographs. PD type and apex were determined: $\mathrm{CS}=$ cervical, $\mathrm{CTJ}=$ cervicothoracic junction, $\mathrm{TH}=$ thoracic, $\mathrm{SP}=$ spinopelvic. Patients were evaluated if the surgery included the PD based on the lowest instrumented vertebra (LIV) - CS: LIV=C7, CTJ: LIV=T3, TH: LIV=T12. Paired t-tests compared post-op RC in unfused segments in PD groups.

Results: PD distributions in 84 CSD patients: CS=33 (40.2\%), $\mathrm{CTJ}=12$ (14.6\%), TH=26 (31.7\%), SP=11 (13.4\%). PD groups were similar in surgical approach and osteotomy type ( $\mathrm{p}>05$ all cases), but $\mathrm{CS}$ and TH drivers differed in levels fused (8.00 vs. 11.39, $\mathrm{p}=.032)$. Construct ranges for PD type: $\mathrm{CS}=\mathrm{C} 2-\mathrm{T} 3, \mathrm{CTJ}=\mathrm{C} 3-\mathrm{T} 6, \mathrm{TH}=\mathrm{C} 3-\mathrm{T} 8$. If the fusion included CTJ driver ( $\mathrm{LIV}=\mathrm{T} 3 ; \mathrm{n}=7,63.6 \%$ ), patients had a reciprocal LL decrease $\left(59.86^{\circ}\right.$ vs. $\left.54.71^{\circ}, \mathrm{p}=.039\right)$. Fusions including $\mathrm{TH}$ drivers (LIV $=\mathrm{T} 12 ; \mathrm{n}=20,76.9 \%$ ) experienced $\mathrm{RC}$ in PI-LL $\left(-5.42^{\circ}\right.$ vs. $\left.-1.11^{\circ}, \mathrm{p}=.018\right)$, $\mathrm{LL}^{\circ}\left(58.05^{\circ}\right.$ vs. $\left.53.95^{\circ}, \mathrm{p}=.017\right)$, and cranial parameters: $\mathrm{C} 1$ slope $\left(17.68^{\circ}\right.$ vs. $\left.0.11^{\circ}, \mathrm{p}<.001\right), \mathrm{C} 2-0$ angle $\left(46.72^{\circ}\right.$ vs. $\left.37.28^{\circ}, \mathrm{p}=.003\right)$, and MGS $\left(-27.00^{\circ}\right.$ vs. $\left.-2.60^{\circ}, \mathrm{p}=.010\right)$. Patients whose fusion did not include the PD did not display improvements in global/ spinopelvic alignment ( $\mathrm{p}>.05)$, but had lower $\mathrm{Cl}\left(3.00^{\circ}\right.$ vs. $-10.28^{\circ}$, $\mathrm{p}=.001)$ and $\mathrm{C} 0\left(1.73^{\circ}\right.$ vs. $\left.-10.28, \mathrm{p}=.001\right)$ angles.

Conclusion: Cervical and thoracic were the main CSD drivers. Cranial and thoracolumbar RC occurred after CSD surgery when the construct included the primary driver. RC that trend toward normalized values depended on PD apex and levels fused - considering these factors is important for optimizing CD sagittal alignment and planning.

206. Outcomes of Operative Treatment for Adult Cervical Deformity: A Prospective Multicenter Assessment with 1-Year Follow-up

Justin S. Smith, MD, PhD; Tamir T. Ailon, MD, MPH; Christopher I. Shaffrey, MD, FACS; Han Jo Kim, MD; Gregory M. Mundis; Munish C. Gupta, MD; Eric Klineberg, MD; Frank Schwab, MD, PhD; Virginie Lafage, PhD; Renaud Lafage; Peter G. Passias, MD; Themistocles Protopsaltis, MD; Brian J. Neuman, MD; Alan H. Daniels, MD; Justin K. Scheer, MD; Alex Soroceanu; Khaled 
Kebaish, MD; Robert Hart, MD; Michael F. O'Brien, MD; Douglas C. Burton, MD; Vedat Deviren, MD; Todd Albert, MD; K. Daniel Riew, MD; Shay Bess, MD; Christopher P. Ames, MD; International Spine Study Group

Introduction: There remains a paucity of high-quality studies that assess outcomes of surgical treatment for adult cervical deformity (ACD). Our objective was to assess outcomes following surgical treatment for ACD based on a prospective multicenter consecutive case series.

Methods: Surgically-treated ACD patients eligible for 1-year follow-up were identified from a prospectively collected multicenter database. Baseline deformity characteristics, surgical parameters, and 1-year outcomes were assessed. Outcome measures included: Neck Disability Index (NDI), neck pain numeric rating scale (NRS) score, and EQ-5D.

Results: Of 77 ACD patients, 55 (71\%) had 1-yr follow-up (64\% women, mean age 61 years, mean Charlson Comorbidity Index [CCI] of 0.6 , previous surgery in $44 \%$ ). Diagnoses included: cervical sagittal imbalance (62\%), cervical kyphosis $(60 \%)$, proximal junctional kyphosis $(8 \%)$ and coronal deformity (10\%). Posterior fusion was performed in $85 \%$ (mean levels $=10$ ), and anterior fusion was performed in $29 \%$ (mean levels $=5$ ). Three-column osteotomy was performed in $24 \%$ of patients. At 1-year following surgery, ACD patients had significant improvement in NDI (50.5 to 38.0, $\mathrm{p}<0.001$ ), neck pain NRS (6.9 to $4.3, \mathrm{p}<0.001)$, EQ-5D index (0.51 to 0.66 , $\mathrm{p}<0.001$ ), and EQ-5D subscores: mobility (1.9 to $1.7, \mathrm{p}=0.019)$, usual activities (2.2 to $1.9, \mathrm{p}=0.007)$, pain/discomfort ( 2.4 to $2.1, \mathrm{p}<0.001)$, and anxiety/depression (1.8 to $1.5, \mathrm{p}=0.014$ ). A nonsignificant trend favoring improvement was observed for EQ-5D self-care (1.5 to 1.3, $\mathrm{p}=0.070$ ). Compared with patients that achieved 1-year follow-up, those lost to follow-up did not differ significantly with regard to age, gender, CCI, number of fused vertebral levels, or baseline NDI, neck pain NRS or EQ-5D scores.

Conclusion: Based on a prospective multicenter series of ACD patients, surgical treatment significantly improved multiple measures of pain and function, including NDI, neck pain NRS score, and EQ-5D. Further follow-up will be necessary to assess the durability of these surgical procedures and the resulting improved outcomes.

\section{Three-column Osteotomy For Correction of Cervical and Cervicothoracic Deformities: Alignment Changes and Early Complications in a Multicenter Prospective Series of 24 Patients}

Justin S. Smith, MD, PhD; Christopher I. Shaffrey, MD, FACS; Renaud Lafage; Virginie Lafage, PhD; Frank Schwab, MD, PhD, Han Jo Kim, MD; Justin K. Scheer, MD; Themistocles Protopsaltis, MD; Peter G. Passias, MD; Gregory M. Mundis; Robert Hart, MD, Brian J. Neuman, MD; Eric Klineberg, MD; Richard A. Hostin, MD; Shay Bess, MD; Vedat Deviren, MD; Christopher P. Ames, MD; International Spine Study Group

Introduction: Three-column osteotomy (3CO; pedicle subtraction osteotomy [PSO] or vertebral column resection VCR]) can provide powerful alignment correction and disability improvement in adult cervical deformity (ACD). Our objective was to prospectively assess cervical alignment improvement and complications in ACD patients treated with $3 \mathrm{CO}$.

Methods: ACD patients treated with 3CO with minimum 90-day follow-up were identified from a prospectively-collected multicenter, consecutive patient ACD database. Complications within 90-days of surgery were collected and classified as minor or major. Standing radiographs were obtained at baseline and 90-days.

Results: All 24 ACD patients treated with 3CO (15 PSO/9 VCR) achieved 90 -day follow-up ( $71 \%$ women, mean age 62 years, previous surgery in 54\%). Diagnoses included: cervical sagittal imbalance $(92 \%)$, cervical kyphosis (38\%), proximal junctional kyphosis (17\%), coronal deformity $(8 \%)$ and distal junctional kyphosis (4\%). The mean number of posterior fusion levels was 13 . The most common 3 CO levels were T1 (38\%), T2 (29\%) and T3 (21\%). A total of 25 (19 major/6 minor) complications were reported, with $14(58 \%)$ and $6(25 \%)$ patients affected, respectively. Overall, 17 (71\%) patients had at least one complication. The most common complications were excessive blood loss $(>1.7 \mathrm{~L}, 25 \%)$, neurologic deficit $(17 \%)$, distal junctional kyphosis (DJK, 8\%), wound infection (13\%), and cardiorespiratory failure $(8 \%)$. Four $(17 \%)$ patients required re-operation within 90-days ( 2 for nerve root motor deficit, 1 deep wound infection, 1 implant pain/prominence). Cervical sagittal alignment improved significantly following 3CO: cervical lordosis $\left(\mathrm{CL}, 3^{\circ}\right.$ to $\left.13^{\circ}, \mathrm{p}=.031\right), \mathrm{C} 2-7$ sagittal vertical axis (66 to $44 \mathrm{~mm}$, $\mathrm{p}<.001)$, and $\mathrm{T} 1$ slope minus CL $\left(46^{\circ}\right.$ to $\left.27^{\circ}, \mathrm{p}<.001\right)$.

Conclusion: Among $24 \mathrm{ACD}$ patients treated with $3 \mathrm{CO}$, cervical sagittal alignment improved significantly following surgery. Overall, 17 (71\%) patients had at least one complication (19 major/6 minor). The most common complications were excessive blood loss $(>1.7 \mathrm{~L})$, neurologic deficit, DJK, wound infection and cardiorespiratory failure.

208. Impact of Multi-Level Interbody Fusion in the Treatment of Severe Adult Scoliosis (Thoracolumbar Coronal Cobb Angle $>\mathbf{5 0}$ Degrees)

Michael Y. Wang, MD, FACS; Joseph M. Zavatsky, MD; Paul Park, MD; Dean Chou, MD; Adam S. Kanter, MD; Juan S. Uribe, MD; Pierce D. Nunley, MD; Robert Eastlack, MD; Behrooz A. Akbarnia, MD; Neel Anand, MD; Stacie Nguyen, BS, MPH; Praveen V. Mummaneni, MD; Gregory M. Mundis, MD; International Spine Study Group

Introduction: The benefits of surgery in the treatment of severe adult spinal deformity (ASD) have been documented. Specific interbody fusion (IBF) techniques compared to all-posterior technique without IBF have yet to be analyzed.

Methods: Patients with ASD having thoracolumbar coronal Cobb angles $>50$, without primary thoracic curves or 3-column osteotomy, and 2-year follow up were included. Patients were split into 2 groups, IBF vs No IBF. IBF included either percutaneous or open pedicle screws. Open: all-posterior without interbody fusion. Differences in demographic, radiographic, and clinical parameters were analyzed. Subgroup analysis of IBF (ALIF vs TLIF vs LLIF) was performed.

Results: 420 patients met inclusion criteria, of those 165 were identified and 118 had full data for analysis (88 IBF patients; 30 No IBF). IBF were older, had higher BMI, and worse preop ODI $(\mathrm{p}<0.05)$. There were differences in pre to post: PT $(-3 \mathrm{vs} 3, \mathrm{p}=0.01)$, PI-LL (-13.5 vs $5, \mathrm{p}<0.001)$, LL (13.6 vs $-3.6, \mathrm{p}<0.001)$, SVA ( -36.1 vs $0.6, \mathrm{p}=0.002)$ and ODI (-17.9 vs $-7.7, \mathrm{p}=0.024)$ in the IBF vs No IBF. IBF had more staged procedures, blood transfusions, iliac fixation, longer OR time, and LOS $(\mathrm{p}<0.05)$. Subgroup analysis revealed LLIF approach had less iliac fixation, EBL, transfusions, and posterior segments fused, while achieving the greatest coronal correction $(\mathrm{p}<0.05$; Table 1$)$.

Conclusion: IBF resulted in significant improvements in all spinopelvic parameters including SVA. Subgroup analysis of the IBF Group revealed the LLIF technique had significantly less EBL, transfusions, and posterior segments fused, while achieving the greatest coronal correction compared to ALIF and TLIF. 
209. Does Concave Versus Convex Approach Matter When Using Lateral Lumbar Interbody Fusion for Adult Scoliosis?

Joseph M. Zavatsky, MD; Adam S. Kanter, MD; Gregory M. Mundis, MD; Zachary Tempel, MD; Stacie Nguyen, BS, MPH; Dean Chou, MD; Paul Park, MD; Juan S. Uribe, MD; Michael Y. Wang, MD, FACS; Neel Anand, MD; Robert Eastlack, MD; Praveen V. Mummaneni, MD; David O. Okonkwo, MD, PhD; International Spine Study Group

Introduction: Minimally-invasive (MIS) lateral lumbar interbody fusion (LLIF) is an effective adjunct in adult degenerative scoliosis (ADS) surgery. LLIF approaches from the concavity or convexity have inherent approach-related risks and benefits. We analyzed LLIF approach-related complications and radiographic and clinical outcomes in patients with ADS.

Methods: A multicenter retrospective review of a MIS adult spinal deformity database with the following inclusion criteria: age $>18$ years, and one of the following: coronal Cobb angle $(C C A)>20$, $\mathrm{SVA}>5 \mathrm{~cm}, \mathrm{PI}-\mathrm{LL}>10$ and/or PT $>20$. Patients were divided into two groups determined by LLIF approach: concave or convex.

Results: 420 patients met inclusion criteria of the database, of those, 165 had complete 2-year data. Of these, 63 patients underwent MIS reconstruction (cMIS: LLIF alone and LLIF + MIS pedicle screws), 40 from the concavity and 23 from the convexity side. No differences between groups were noted in demographic, and preop or post op radiographic parameters. EBL, length of stay and, and OR time were similar. Both groups equally treated L4-5 via LLIF and chose a similar LIV. Overall complications were similar between groups. There were no significant differences in neurologic, vascular, or visceral complications. There were 8 ( 3 major) neurologic complications in the concavity group of which 1 required reoperation for decompression and one revision of a pedicle screw. One (minor; LFCN palsy) neurologic complication occurred in the convexity group $(\mathrm{p}=0.09)$. Both groups experienced significant improvement in ODI, VAS back and leg (all $\mathrm{p}<0.05)$, with no difference between groups.

Conclusion: Patients undergoing cMIS surgery for adult degenerative scoliosis had equivalent complication rates, clinical and operative, with concave or convex LLIF approaches. Radiographic and clinical outcomes improved regardless of side of LLIF approach.

210. Patients with High Pelvic Tilt Achieve the Same Clinical Success as Those with Low Pelvic Tilt After Minimally Invasive Deformity Surgery

Gregory M. Mundis, MD; Jacob Januszewski, DO; Juan S. Uribe, MD; Michael Y. Wang, MD, FACS; Neel Anand, MD; David O. Okonkwo, MD, PhD; Stacie Nguyen, BS, MPH; Praveen V. Mummaneni, MD; Pierce D. Nunley, MD; Paul Park, MD; Adam S. Kanter, MD; Joseph M. Zavatsky, MD; Robert Eastlack, MD; Behrooz A. Akbarnia, MD; International Spine Study Group

Introduction: Pelvic tilt (PT) has been shown to correlate with HRQOL. The effect of PT in minimally invasive spine surgery for adult spinal deformity (ASD) has not been well studied. We present a comparison of clinical outcomes among ASD patients with high and low PT.

Methods: Retrospective review of a multicenter MIS ASD database was performed. The inclusion criteria for the database were: age $>18$, and one of the following: coronal Cobb angle (CCA) $>20$, SVA $>5 \mathrm{~cm}, \mathrm{PI}-\mathrm{LL}>10$ or PT $>20$. Patients undergoing circumferential minimally invasive (cMIS: lateral interbody fusion with MIS screw placement) correction with a minimum 2-year follow-up were included, and stratified into two groups based on the Schwab classification of PT: High PT $(>30)$ and Low PT $(<20)$.

Results: Among 420 patients in the database, 165 had complete 2-year data. Of the 165 eligible, 43 patients met criteria of the present study for analysis (25 Low, 18 High PT). The High PT group had higher pre-op PI-LL mismatch (32.1 vs. 4.7; $<<0.001)$ (Table 1). At last follow-up, 77\% (14) of patients in High PT Group had continued PI-LL mismatch compared to $40 \%$ (10) in the Low PT Group $(\mathrm{p}<0.006)$. There was a difference in the High and Low PT groups in terms of postop changes of PT $(-3.9 \mathrm{v} 1.9)$, LL $(8.7 \mathrm{v} 0.5)$ and PI-LL $(-9.5 \mathrm{v} 0.1)$. HRQOL were similar preoperatively with the patients in the High PT group having more back and leg pain. Postoperatively, HRQOL (ODI and VAS back/leg) were significantly improved in both groups $(\mathrm{p}<0.05)$.

Conclusion: The degrees of PT seems to be correlated with PI, SVA and PI-LL mismatch. The higher PT, the lower LL, and higher PI and PI-LL mismatch. However, patients with high preoperative PT treated with cMIS techniques had less radiographic success without compromising the clinical outcomes. Further investigation is required to explain the role of $\mathrm{PT}$ when treating ASD patients using MIS techniques.

\section{Advanced Lateral Approaches with Hyperlordotic Interbody Grafts Can Correct Spinopelvic Mismatch While Avoiding the Blood Loss Associated with Pedicle Subtraction Osteotomy}

\section{Jean-Christophe A. Leveque, MD; Vijay Yanamadala, MD; Quinlan} Buchlak; Rajiv Sethi, MD

Introduction: Pedicle subtraction osteotomy (PSO) achieves excellent correction of sagittal plane deformities but is associated with?operative morbidity including high estimated blood loss (EBL) and neurological injury. Anterior column realignment (ACR) with lateral graft placement and sectioning of the anterior longitudinal ligament allows restoration of lumbar lordosis through placement of interbody cages with fixed lordosis angles.

Methods: Retrospective chart review of all cases involving a lumbar PSO or lateral hyperlordotic ACR (XLH-ACR) between 2010-2015. Demographics, spinopelvic parameters, EBL, operative time, and LOS were gathered in the course of this chart review. Statistical significance was $\mathrm{p}<.05$ using a two-tailed t-test.

Results: 14 patients underwent PSO and posterior fusion. 13 patients who underwent multilevel XLH-ACR with posterior fusion. Mean EBL was significantly lower in the XLH-ACR group, measuring less than $50 \%$ of the mean blood loss in the PSO group (1466cc vs 2910cc, $\mathrm{p}<.01$ ). The total correction in lumbar lordosis was equivalent between the two groups (35 degrees in PSO group, 30 degrees in XLH-ACR group, $\mathrm{p}>$.05), as were the preoperative PI-LL mismatch (33 degrees in each group, $\mathrm{p}>.05$ ) and the postoperative PI-LL mismatch (- 0.6 degrees in the PSO group, +0.8 degrees in the XLH-ACR, $\mathrm{p}=.05)$.Total operative time and LOS were not significantly different between the two groups.

Conclusion: This is the first direct comparison of the ACR technique with the PSO in adult spinal deformity correction. We demonstrate that the ACR provides equivalent deformity correction with significantly reduced blood loss in patients with a previously unfused spine. This technique provides a powerful means to avoid the PSO in select patients who require spinal deformity correction. The reduced morbidity of the ACR may make spinal deformity surgery accessible to a broader range of patients. Future studies of ACR will be necessary to elucidate the long-term outcomes of this technique compared to the PSO. 
212. Scoliotic Deformity Correction in 107 Patients Undergoing Single-Level Minimally Invasive Transforaminal Lumbar Interbody Fusion

Vikram Chakravarthy, MD; Namath Syed Hussain, MD, MBA; Mick J. Perez-Cruet, $M D, M S$

Introduction: There exists substantial evidence to support lumbar fusion procedures in cases of spondylolysis, spondylolisthesis, and in other cases of sagittal deformity. However, coronal scoliotic deformity can also be a cause of low back and leg pain in a certain subset of patients.

Methods: 350 patients who underwent MITLIF were followed for 7 consecutive years. This was pared down to 107 patients who had preoperative and postoperative radiographs that exhibited a clear coronal deformity that correlated with the patient's symptomotology. Dynamic radiographs were analyzed using a three-point angle measurement tool through EasyViz viewer program. Cobb angles were recorded preoperatively and postoperatively along with a validated full set of Health-related Quality of Life (HRQL) Measures, including Visual Analog Scale, Oswestry Disability Index, and SF-36.

Results: 66 females and 41 males were treated (average age 68.6, range 39 - 92). Levels fused included L1-L2 $(n=1,1 \%), \mathrm{L} 2-\mathrm{L} 3(\mathrm{n}=10$, 9.3\%), L3-L4 ( $n=16,15 \%)$, L4-L5 ( $n=75,70 \%)$ L5-S1 $(n=1,1 \%)$, or multi-level fusion $(\mathrm{n}=4,3.7 \%)$. Average Cobb angle decreased from 9.47 preoperatively to 7.54 postoperatively $(\mathrm{p}<0.05$ ). Cobb angles ranged from $0.7 \%-43.2 \%$ preoperatively, and $0.7 \%-34.1 \%$ postoperatively. There was a statistically significant correlation between Cobb angle improvement and HRQL measure improvement. Patients also exhibited an age related trend, with average Cobb angles increasing as the patient ages.

Conclusion: Cobb angles improved after MITLIF in 94\% $(n=101)$ of patients and this improvement correlated with an improvement on symptom questionnaires. MITLIF resulted in a high rate of spinal fusion, and a low rate of reoperation. Cobb angles were reduced $21 \%$ on average with just a single-level fusion, supporting the consideration of a focused single-level fusion versus a large multilevel fusion in cases of coronal deformity, especially in the elderly.

213. Treatment of the Fractional Curve with Circumferential Minimally Invasive (cMIS) Interbody Versus Oery Without Interbody Fusion: An Analysis of Surgical Outcomes

Dean Chou, MD; Praveen V. Mummaneni, MD; Pierce D. Nunley, MD; Joseph M. Zavatsky, MD; Robert Eastlack, MD; David O. Okonkwo, MD, PhD; Michael Y. Wang, MD, FACS; Paul Park, MD; Juan S. Uribe, MD; Neel Anand, MD; Vedat Deviren, MD; Behrooz A. Akbarnia, MD; Stacie Nguyen, BS, MPH; Gregory M. Mundis, MD; International Spine Study Group

Introduction: The fractional curve of adult spinal deformity (ASD) can be difficult to treat. We sought to evaluate the outcomes of patients whose fractional curves were treated with either cMIS or open techniques without interbody (IB).

Methods: Retrospective review of 2 multicenter ASD databases (Open- prospective; MIS- retrospective), with the following inclusion criteria: age $>18$ years with fractional curves $>10,=3$ levels of instrumentation, and one of the following: coronal Cobb angle $(\mathrm{CCA})>20$, PI-LL $>10, \mathrm{PT}>20, \mathrm{SVA}>5 \mathrm{~cm}$. Fractional curve was measured from S1 and the last vertebrae of the lower Cobb. Only fractional curves of 10 degrees or greater were evaluated. In the OPEN cohort, only patients without IB were included.

Results: 888 patients met inclusion criteria for the database, of which 508 had complete 2 - year data. 118 patients had their fractional curves treated, and after propensity matching for levels treated, 40 patients were eligible with either cMIS (20) or with open (20) surgery. Preop fractional curve was 18 in both groups and corrected to 6.9 in cMIS and 8.5 in open ( $>00.05)$. cMIS patients had a smaller postop coronal Cobb (12.5 vs 24.3; $=0.02)$ and lower EBL (809cc vs $2299 \mathrm{cc} ; \mathrm{p}=0.002)$. Open patients had a higher SVA change (-1.96 vs $+1.32 \mathrm{~cm} ; \mathrm{p}=0.036)$, more pelvic fixation $(55 \%$ vs $15 \% ; \mathrm{p}=0.008)$, Both groups had similar pre and postop VAS leg pain with no difference between groups at 2 years (change VAS Leg -4.4 vs $-2.2 ; p=0.06$ ). There was no significant difference in change of Cobb angle, ODI, PI-LL, LL, or VAS Back.

Conclusion: Treating fractional curves with cMIS surgery improved leg pain and comparable correction to the open group, and when matched for levels treated, the outcomes remained similar.

214. Re-admission, Re-operation, and Patient Reported Outcomes After Lumbar Fusion Surgery for Spondylolisthesis in 480 Patients from the QOD Registry - Supported by the NREF Regis Haid Fund

Erica Bisson; Mohamad Bydon, MD; Steven D. Glassman; Kevin T. Foley, MD, FACS; Jonathan Slotkin, MD; Eric A. Potts, MD; Mark Edwin Shaffrey, MD, FACS; Domagoj Coric, MD; John J. Knightly, MD; Paul Park, MD; Kai-Ming G. Fu, MD, PhD; Anthony L. Asher, MD, FACS; Andrew Kai-Hong Chan, MD; Michael S. Virk, MD, PhD; Praveen V. Mummaneni, MD

Introduction: The American Association of Neurological Surgeons launched the National Neurosurgery Quality and Outcomes Database (QOD), a prospective longitudinal registry utilizing patient reported outcome instruments and includes data to measure the safety and quality of spinal surgery. In the present study, the authors analyzed 12-month outcomes data for patients undergoing lumbar fusion surgery for grade 1 degenerative spondylolisthesis.

Methods: The prospective QOD dataset from top enrolling sites was retrospectively queried and we found 480 patients undergoing elective spine surgery for one, two, or three level degenerative grade 1 lumbar spondylolisthesis. Baseline, 3-month, and 12-month follow-up readmission rates, re-operation rates, and patient reported outcomes (ODI and back and leg pain NRS) were collected. The absolute differences between 12-month post-operative and baseline ODI and back and leg pain NRS scores were evaluated.

Results: The 30-day readmission rate was 3.3\%. The 90-day readmission rate was $4.6 \%$. The 30 -day re-operation rate was $3.1 \%$. The baseline ODI scores differed from the 12-month scores by an absolute difference of $12.1 \pm 8.8(\mathrm{p}<.001)$. The baseline back and legpain NRS scores differed from the 12-month scores by an absolute difference of $3.9 \pm 3.1$ and $4.0 \pm 3.6$, respectively $(p<.001)$.

Conclusion: This registry is unique as it has patient data acquired directly from the surgeons' office. Lumbar fusion surgery for grade 1 degenerative lumbar spondylolisthesis is associated with low 30-day re-operation rates and 30- and 90-day readmission rates. Fusion surgery was associated with significant improvements in patientreported outcomes at one-year follow up in the N2QOD registry.

215. New Lamina Spacer that can be Applied to Both of Open Door and Double Door Cervical Laminoplasty and Gives Consecutive Bone Union Between Spacer and Opened Lamina Clinical Outcomes and Bone Union of the Opened Lamina and Spacer

Futoshi Suetsuna, MD

Introduction: HA lamina spacer has been used in cervical laminoplasty. However, reports on bone absorption of opened lamina or displacement of HA spacers were seen. The purpose is to evaluate the clinical and radiographic outcomes of laminoplasty using titanium 
lamina spacer (TLS) that bone union between opened lamina and spacer can be expected.

Methods: 30 patients with an average age of 65.4 years that underwent laminoplasty using TLS for cervical myelopathy were retrospectively reviewed. The average follow up period was 36 months $(>12$ months). Radiographic parameters included bone unions of TLS and opened lamina and lateral gutters, cervical range of motion (ROM) and lordosis. Clinical parameters included surgical outcomes using JOA scores, neck pain and complications. TLS has a gap to allow insertion of grafting bones. The graft filled in the gap involved only local bones (16 cases), only collagen hybrid hydroxyapatite (5 cases) and mixing of local bones and collagen hybrid hydroxyapatite ( 9 cases). Bone union was determined from continuous bone density of the opened lamina and new bone inside the gap using CT in one year after surgery.

Results: Bone union of lateral gutters was obtained in all cases. Consecutive bone union between TLS and opened lamina was obtained in $100 \%$ cases of local bone group, $60 \%$ of collagen hybrid group and $78 \%$ of mixed group. The average preoperative and postoperative cervical ROMs and lordosis were 39.9 degrees and 19.6 degrees and 29.0 degrees and 18.4 degrees respectively. The average preoperative and postoperative JOA scores were 10.6 points and 14.2 points. Recovery rate using was $56.2 \%$.

Conclusion: These clinical results were equivalent to that of our study using HA spacer. TLS that leads to bone union between spacer and opened lamina and has sufficient fixation force in biomechanical study is a safe and useful spacer for laminoplasty.

\section{Voice and Swallowing Outcomes Following Re-Operative ACDF Surgery with a Two Surgical Team Approach}

Matthew Erwood; Beverly C. Walters, MD, MSc, FRCS(C), FACS; Timothy Connolly; Amber S. Gordon, MD; William Carroll; Bonita Agee; Mark N. Hadley, MD, FACS

Introduction: Dyphagia and vocal cord palsy (VCP) are common complications after ACDF surgery. The incidence of VCP is reported to be as high as $22 \%$, while the incidence of dysphagia ranges widely, with estimates up to $71 \%$. This study set out to investigate the incidence of voice and swallowing disturbance before and after re-operative ACDF procedures using a two-team operative approach with comprehensive pre-and post-operative assessment of swallowing and direct vocal cord visualization.

Methods: A convenience sample of sequential patients identified as requiring re-operative ACDF surgery at the University of Alabama at Birmingham (UAB) were enrolled in a prospective, non-randomized study during the period May 2010 until July 2014. Sixty-seven patients undergoing revision ACDF surgery were enrolled using a two-team approach with neurosurgery and ENT. Dysphagia was assessed both preoperatively and post-operatively using MDADI and FEES, while vocal cord palsy was assessed using direct visualization with videolaryngostroboscopy (VLS).

Results: Five patients (7.5\%) developed a new post-operative temporary VCP after re-operative ACDF surgery. There were no new instances of permanent VCP. Twenty-five subjects had a new swallowing disturbance detected on FEES compared with their baseline assessment, with most being mild and requiring no intervention. $60 \%$ of patients showed a decrease in their post-op MDADI scores, particularly within the physical subset.

Conclusion: Two-team approach to re-operative ACDF was safe and effective, with no new cases of VCP on post-op VLS. Dysphagia rates as assessed through MDADI scale and FEES were in keeping with other published reports.

\section{Risk Factors for Durotomy in Elective Spine Surgery}

Panagiotis Kerezoudis; Meghan Murphy, MD; Mohammed Ali Alvi, MD; Patrick R. Maloney, MD; Lorenzo Rinaldo, MD, PhD; Daniel Levi Shepherd, MD; Daniel S. Ubl, BA; Elizabeth B. Habermann, PhD; Mohamad Bydon, MD

Introduction: Incidental durotomy(ID) is a known complication in elective spine surgery. Prior studies focus on smaller cohorts from single institutions with homogenous patient populations. Previously established risk factors include increased patient age, degenerative pathologies, and revision surgery. Using a large, multicenter database, this study evaluated an elective spine surgery population for ID risk factors using the American College of Surgeons National Surgical Quality Improvement Program (ACS-NSQIP) database.

Methods: A retrospective cohort analysis queried NSQIP for patients with degenerative spinal diagnoses undergoing elective spinal surgery from 2010-2014. Multivariable logistic regression analysis interrogated for independent risk factors of durotomy.

Results: Of 104,930 patients an ID occurred in $0.6 \%$ of cases. On adjusted analysis the following factors were independently associated with durotomy: ankylosing spondylitis vs. intervertebral disc disorders (OR 4.6, 95\% CI: 1.1-19.4), 2-4(OR 1.8, 95\% CI: 1.5-2.2) or $5+(\mathrm{OR} 1.8,95 \%$ CI: 1.4-2.5) vs. 1 level, orthopedist vs. neurosurgeon(OR 1.6, 95\% CI: 1.4-1.9), combined surgical approach (OR 3.5, 95\% CI: 1.9-6.6) or posterior approach (OR 8.1, 95\% CI: 4.913.4) vs. anterior approach, age groups $85+(\mathrm{OR} 2.2,95 \%$ CI: 1.4-3.5), 75-84 (OR 1.5, 95\% CI: $1.2-1.9$ ), or $65-74$ (OR 1.4, 95\% CI: $1.1-1.7$ ) vs. $<65$, obesity $(\mathrm{BMI}=30)(\mathrm{OR} 1.2,95 \% \mathrm{CI}: 1.0-1.4)$, corticosteroid use(OR 1.5 95\% CI: 1.1-2.1), and preoperative platelet count $<150$ (OR 1.4 95\% CI: 1.0-1.9).

Conclusion: This large, multicenter study identifies several risk factors for ID in the elective spine surgery population. Novel risk factors identified include surgeon specialty, corticosteroid use, thrombocytopenia, and ankylosing spondylitis. The results of this analysis provide further information for surgeons to use both in operative planning and in preoperative counseling when discussing of the risk of ID.

\section{The Extent of Lumbar Decompression and its Effect on Postoperative Morbidity}

Mohammed Ali Alvi, MD; Patrick R. Maloney, MD; Brandon A. McCutcheon, MD, MPP; Meghan Murphy, MD; Elizabeth B. Habermann, PhD; Cynthia Crowson; Daniel Levi Shepherd, MD; Panagiotis Kerezoudis; Jennifer Grauberger; Mohamad Bydon, MD

Introduction: Decompressive laminectomy without fusion is one of the most common surgical spine procedure for treating lumbar spondylosis. In the absence of instability, decompressive laminectomies provide symptom relief with restoration of functional movement in most patients. The extent of the decompression, by number of vertebral levels, and its effects on postoperative morbidity were assessed using a national surgical database.

Methods: This study is a retrospective cohort analysis utilizing the 2011-2013 American College of Surgeons National Surgical Quality Improvement Program (ACS-NSQIP). Patients admitted from home with degenerative spine disease for lumbar decompression without fusion were included. Thirty-day outcomes and operative characteristics were compared with regard to the number of vertebral levels decompressed using chi-square and Kruskal-Wallis tests. Multivariable logistic regression analysis was used to interrogate whether the extent of lumbar decompression was significantly associated with adverse discharge disposition, 30-day readmission, or any minor complication.

Results: A total of 8744 patients were identified and stratified into the following cohorts by the number vertebral levels of decompression: 
one, two, and three or more (3+) levels. Univariate analysis showed that $3+$ levels was significantly associated with increased age, increased BMI, non-smoking status, functional dependency , hypertension requiring medications, steroid use for chronic conditions, decreased glomerular filtration rate, and higher ASA score (all $\mathrm{p}<0.05$ ). Operative and outcome measures associated with $3+$ levels included operative time, perioperative blood transfusions, any major morbidity, length of hospital stay, readmissions within 30 days, return to the operating room, and non-home discharge(all $\mathrm{p}<0.05$ ). On multivariable analysis with single level surgery as the reference, increased levels of surgery were significantly associated with discharge location other than home (two levels OR 1.5, 95\% CI 1.29-1.85) (3+ levels OR $1.75,95 \%$ CI $1.40-2.20)$ but not 30 day readmission or any minor complication (both $\mathrm{p}>0.05$ ).

Conclusion: In patients undergoing lumbar decompression, increasing the number of vertebral levels was significantly associated with postoperative morbidity. Increased number of levels was associated with a significantly higher odds of discharge to a location other than home. Postoperative morbidity risk stratification and disposition merit discussion during preoperative counseling, especially when multilevel decompressions are being considered.

\section{Safety of Use of Autologous Concentrated Bone Marrow} Aspirate in Spinal Fusion Procedures

Lara Walsh Massie, MD; Kelly Tundo, BS, CCRP; Lonni Schultz, PhD; Mokbel K. Chedid, MD, FACS

Introduction: Concentrated bone marrow aspirate (cBMA) is an alternative source for autologous stem cells and endogenous factors that are found in iliac crest graft. Bone marrow can be aspirated through the same incision and prepared into cBMA during surgery. This study characterizes the safety of the cBMA technque and its application in spine fusion.

Methods: Medical records of 150 consecutive patients undergoing spinal fusion with cBMA between 2008-2011 were reviewed for any occurrence of adverse events (AEs). These events were categorized as related, possibly related, or unrelated to use of the cBMA.

Results: Seventy-four (49\%) of 150 patients reported no AEs. The remaining 76 patients $(51 \%)$ reported 124 total AEs. Back pain and/ or radiculopathy, accounted for $49 \%(\mathrm{n}=61)$ AEs. Ten $(8 \%)$ wound infections and $9(7 \%)$ at another site were documented. Other AEs included 6 deep vein thromboses, 5 ileuses, 3 unintended durotomies, 2 episodes of symptomatic post-operative anemia, 11 unrelated traumas, and 17 exacerbation of other medical condition (ex. anxiety, headaches, asthma). Four patients (3\%) required surgical intervention related to AEs: Two developed adjacent level disease an average of 2.5 years after surgery. One requested removal of hardware. One patient required revision of fusion for pseudarthrosis 35 months after surgery. None of the AEs were definitively related to the harvesting technique or use of cBMA. $76(61 \%)$ were clearly unrelated to the use of cBMA (ex: UTI, exacerbation of medical comorbidities). The remaining $48(39 \%)$ were probably unrelated to the use of cBMA (back pain, anemia)

Conclusion: Harvesting of cBMA from the iliac crest intraoperatively did not cause any definitive adverse events. Use of cBMA offers the benefit of autologous mesenchymal stem cells and endogenous growth factors as a valuable adjunct for use in spinal surgery, especially in patient populations in whom biosynthetic adjuncts are contraindicated.
220. Feasibility of C7 Lateral Mass Screws as a First-Line Fixation Method: A Consecutive Series of Forty Nine Patients Over Seven Years

Kevin T Huang, MD; Yethulsa Fingfing, BA; Muhammad M. Abd-ElBarr, MD, PhD; John Chi, MD, MPH

Introduction: Lateral mass screw fixation has become the most widely acceptable method for posterior fixation in the subaxial spine due to its relative ease of placement and lower risk of neurovascular injury. At the $\mathrm{C} 7$ level, there is still some controversy about the ideal method of fixation due to smaller size of the lateral mass at this level and certain biomechanical studies that have shown lower pullout strengths of $\mathrm{C} 7$ lateral mass screws compared to $\mathrm{C} 7$ pedicle screws. In this retrospective study, we attempt to demonstrate the feasibility of lateral mass screw fixation at $\mathrm{C} 7$, with an emphasis on durability and safety.

Methods: A consecutive series of 49 patients who underwent posterior cervical fusion and c7 lateral mass fixation over the course of 7 years was retrospectively reviewed. Patients were then subsequently tracked for any signs of hardware loosening and any subsequent reoperations. Any other complications, such as wound infections, neurologic injury, reoperations, or unintentional durotomies, were also recorded.

Results: Our cohort consisted of 23 females (42\%) and 32 males $(58 \%)$, with an average age of 61 years at time of surgery (SD: 13.0 years). With an average available follow-up of 25.3 months (SD: 26.2 months), two patients (4.1\%) had evidence of hardware failure, only $1(2.0 \%)$ of which was due to screw loosening at the c7 level. 10 $(20.4 \%)$ patients eventually required reoperation (two for hardware failure, three for wound dehiscence, two for adjacent level disease, two for recurrent tumor growth, and one for an epidural hematoma). No patient experienced any neurologic complications or durotomies.

Conclusion: C7 lateral mass screw fixation can be employed in a variety of pathologies with acceptable long-term rates of hardware failure, neurologic injury, and unintentional durotomy.

221. Limiting the Cranial Extent of Fusion to the Atlas Does not Result Delayed Craniocervical Settling or Occipitocervical Instability After Surgical Decompression for Basilar Invagination

Brice Allen Kessler; Nathan Davis; Joshua E. Loewenstein, MD; Deb A. Bhowmick, MD

Introduction: Occipitocervical fusion is commonly employed for the treatment of basilar invagination due to craniocervical junction instability and the destabilizing effects of adjunctive brainstem decompression surgery. This procedure has significant morbidity associated with loss of physiological function at the craniocervical junction. However, we hypothesized that many of the underlying pathologies causing basilar invagination were due to dysfunction of the atlantoaxial articulation. In those patients, our practice has been to limit the cranial extent of instrumented fusion to the atlas after direct ventral odontoidectomy or indirect decompression performed by atlantoaxial intraarticular graft placement.

Methods: A retrospective review of postoperative imaging and neurological status was performed for patients undergoing limited arthrodesis to the $\mathrm{C} 1$ vertebral body performed at our institution for treatment of symptomatic basilar invagination from 2013-2015.

Results: A total of 16 patients were treated for basilar invagination with atlantoaxial fusion after decompression, with a minimum of 1 year follow-up over the time period stated. No instances of delayed craniocervical settling or $\mathrm{Cl}$ lateral mass displacement was noted on 1 year radiographs for any of the patients. One patient developed self-limited symptomatic occipitocervical adjacent segment disease without neurological sequelae, treated successfully with conservative management. There was no instances of delayed neurological decline. 
There was one instance of immediate postoperative neurological decline that improved upon follow-up.

Conclusion: Limiting arthrodesis to the atlas can be safely performed without delayed adverse sequelae when performing decompressive surgery for basilar invagination.

222. Perioperative Malnutrition and its Relationship to Hospital Course and Clinical Outcomes in Patients Undergoing Surgery for Cervical Myelopathy

Christopher M. Holland, MD, PhD; Jian Guan, MD; Vijay Ravindra, MD, MSPH; Erica Fay Bisson MD, MPH

Introduction: Malnutrition pre- and postoperatively has been associated with various negative outcomes following surgery. Surgical treatment of cervical myelopathy is common, and the impact of perioperative malnutrition in these patients is unclear. We evaluated the association of low prealbumin level, a marker for poor nutrition, with clinical outcomes after surgery for cervical myelopathy.

Methods: We identified patients who underwent anterior cervical discectomy and fusion, anterior cervical corpectomy and fusion, or posterior cervical decompression and fusion for cervical myelopathy over a five-year period. Patients with prealbumin levels measured within 4 days of surgery were included. Prealbumin levels were dichotomized using a 15-mg/dL threshold into low and normal groups. Demographic, clinical, operative, and postoperative parameters were evaluated with univariate and multivariable analyses to assess association with prealbumin levels.

Results: Eighteen patients met criteria for the "low prealbumin" group and 37 for the "normal prealbumin" group. Patients with low prealbumin were significantly more likely to have an extended length of hospitalization (median 6 days, interquartile range 7.5 days vs. median 3 days, interquartile range 2 days, $p<0.001$ ) and to have had postoperative complications ( $44 \%$ versus $3 \%, \mathrm{p}<0.001$ ). Multivariable analysis revealed that only prealbumin category $(\mathrm{p}<0.001)$ and discharge destination $(\mathrm{p}=0.002)$ were associated with prolonged length of stay.

Conclusion: Low prealbumin levels were associated with prolonged length of hospitalization after surgery for cervical myelopathy. Further investigation is needed to clarify this association and to better define the effects of malnutrition in the cervical myelopathy population.

223. Thoracolumbar Arthrodesis of Degenerative Spondylosis with Stereotactic Cortical Screws and Interbody Fusion

Michael Karsy, MD, PhD; Kyrl Cole, BS; Jian Guan, MD; Andrea Archambault Brock; Chad Douglas Cole, MD, MSc

Introduction: The use of cortical screw and interbody fusions has been a relatively new modality in posterior spinal fusion with equivalent long-term fusion rates compared to traditional pedicle screw placement. Cortical screws allow for a reduced surgical exposure and tissue dissection. However, the long-term followup, spinopelvic parameters and patient quality-of-life outcomes have yet to be fully evaluated with this technique.

Methods: A prospective database was screened for patients that underwent cortical screw placement and interbody fixation for degenerative spondylosis from 2013 to 2014 with at least 1 year follow up. Clinical variables, spinopelvic parameters, fusion rates, and quality-of-life metrics were evaluated

Results: A mean age of $60.3 \pm 12.3$ years was seen for 134 patients. A total of 225 interbody implants and 906 screws were placed. The mean number of interbody devices was $1.8 \pm 1.1$ and fusion levels per patient was $3.4 \pm 1.6$. A total of $24(17.9 \%)$ patients had undergone spine fusion or decompression prior to the index case. Postoperative of pelvic tilt was significantly improved $(1.3 \pm 6.1 \mathrm{o}, \mathrm{p}=0.04)$. While mean change in lumbar lordosis was unchanged postoperatively $(-0.5 \pm 9.7, \mathrm{p}=0.6)$, the mean change in pelvic incidence to lumbar lordosis mismatch was significantly decreased postoperatively $(-1.5 \pm 8.1, \mathrm{p}=0.05)$. Mean screw trajectories were calculated for each level. Four patients suffered complications including 2 dural tears, 1 post-op deep vein, 1 readmission, and 1 pseudoarthrosis at 1 year followup. Patients showed a significant improvement in SF-36 and Oswestry Disability Index metrics.

Conclusion: Our series of 134 patients, 906 cortical screw placements, and 225 interbody implants suggest that this approach can confer improvement in spinopelvic parameters, good long term fusion rates and improvement in quality of life metrics with a low complication rate. The additional benefit of smaller incisions with this procedure can reduce blood loss and tissue dissection to conferring good long-term patient outcomes.

\section{A Clinical and Radiological Study of Zero Profile Device for Anterior Cervical Discectomy and Fusion}

Allen Ho, MD; Sayantan Deb, BA; Suzanne Audrey Tharin, MD, PhD; Eric Tranvinh; Stephen Skirboll, MD

Introduction: Zero-profile, stand-alone polyetheretherketone interbody spacers have been developed to minimize soft-tissue irritation and postoperative dysphagia. However, the majority of the literature reports data utilizing the Zero-P (Synthes) implant and while a plethora of other stand-alone spacers have been developed, studies of other specific implants are limited. The object of the present study was to determine the clinical and radiological outcomes for patients who underwent ACDF using the COALITION (Globus Medical) ACDF spacer system.

Methods: Using a prospective surgical database, the authors conducted a retrospective analysis of all patients who had undergone ACDF between January 2011 and June 2011 at a single institution. All patients received a COALITION ACDF spacer system (Globus Medical). Preoperative characteristics and post-operative outcomes in terms of pain and dysphagia and radiographic findings were documented. Radiographs were assessed by an independent radiologist and parameters examined included cervical and segmental Cobb angles, total segmental height, and subsidence (defined as decrease in post-operative segment height $>3 \mathrm{~mm}$ ).

Results: 65 consecutive patients, of which 2 were female, with a mean age of $59.6 \pm 10.1$ years, underwent ACDF ( 88 total operated levels) in the defined study period. The mean clinical follow-up was $11.58 \pm 10.64$ months. $12(18.5 \%)$ of the patients developed some post-operative dysphagia with a mean duration of 2.5 months. Radiological outcome in was assessed at a mean follow-up of 6.84 months. Patients mean preoperative cervical Cobb angle was 10.63, which improved to a post-operative Cobb angle of 13.22 . There was an increase in segmental Cobb angle by $65.55 \%$. Total construct height increased by $9 \%$. $17 \%$ of the patients had some evidence of subsidence at last radiographic follow-up.

Conclusion: This is the first implant specific study of the COALITION ACDF spacer system. We found clinical and radiographic outcomes comparable to those for nonintegrated plate and spacer constructs.

\section{Should Long Segment Cervical Fusions be Routinely Carried into the Thoracic Spine? Multi-center Analysis}

Eeric Truumees, MD; Devender Singh, PhD; Matthew Geck, MD; John K. Stokes, MD

Introduction: While recommendations for caudal "end level" in 
posterior cervical reconstruction remain highly variable, the benefits of routine extension of posterior cervical fusions into the thoracic spine remain unclear. We compared clinical and radiographic outcomes in patients in whom posterior fusions ended in the cervical spine versus those in whom the fusion was extended into the thoracic spine.

Methods: We assembled a multicenter radiographic and clinical database of patients that had undergone 3 or more level posterior cervical fusions for degenerative disease with at least 2 years of postoperative (post-op) follow-ups. Patients were divided into two groups: group I (fusion ending in the cervical spine) and group II (fusion extending into the thoracic spine).

Results: Group I and Group II had 104 and 73 patients, respectively. Mean estimated blood loss (EBL) for group II was significantly higher than group I $(p<0.05)$. Mean operative time $(\mathrm{OR})$ and length of hospital stay (LOS) were comparatively higher ( $p>0.05)$ for group II than group I. There was no significant difference in change in mean cervical lordosis ( 2 wk vs. 2 year post-op) between the two groups $(\mathrm{p}>0.05)$. Similary, there were no significant differences in change in mean C2-C7 sagittal plumbline and T1 slope ( 2 wk vs. 2 year post-op) between the two groups( $\mathrm{p}>0.05)$. Rate of pseudoarthrosis was higher in group I $(21.2 \%)$ than group II $(10.96 \% ; p>0.05)$. There were significant improvements in mean clinical outcomes at 2 years follow ups in both groups but there were no differences between the two groups ( $\mathrm{p}>0.05)$.

Conclusion: Our analyses indicate that both groups had similar clinical and radiographic outcomes. Lower pseudarthrosis rate but higher EBL, OR and LOS in group II suggest that extension of posterior cervical fusions into the thoracic spine still remains debatable. Prospective studies are needed to elucidate optimal means of posterior stabilization in patients with degenerative cervical disease.

226. Expandable vs. Static Inter-body Grafts Reduce Operative Time, Improve Lordosis and Accelerate Return to Work After Fusion for Lumbar Stenosis and Spondylolisthesis

Matthew J. McGirt, MD; Vincent Rossi, MD; Domagoj Coric, MD; Paul Keetae Kim, MD; Kevin Scott Cahill, MD, PhD; Deborah Pfortmiller

Introduction: Inter-body instrumentation (TLIF/PLIF) improves arthrodesis and lumbar lordosis. Static grafts have become standard of care. Expandable inter-body grafts allow for potential to generate increased lordosis while facilitating less disruptive placement. We set out to determine if expandable grafts were associated with improved lordosis and outcomes compared to static grafts.

Methods: All TLIF/PLIF cases for lumbar stenosis and spondylolisthesis prospectively entered into the National Neurosurgery Quality and Outcomes Database (N2QOD) from our institution between 2011-2015 were analyzed. Patients were stratified based on whether a static PEEK inter-body versus an expandable inter-body graft was used. Post-fusion lumbar lordosis (L1-S1) - pelvic incidence (PI) mismatch was compared between cohorts on standing lateral X-rays. Propensity score matching, Independent t-tests, chi-square tests were used to compare radiographic, peri-operative, and one-year outcomes.

Results: 230 patients with one-year reported outcomes were available for review (188 expandable vs 42 static). In unadjusted comparison, expandable grafts were associated with reduced operative times and a trend of reduced blood loss, Table 1. For single-level fusion, LL/PI mismatch was similar $(+3$ vs +5$)$ between cohorts. For two-level $(+8$ vs +14$)$ and three-level fusion $(+8$ vs +15$)$, expandable grafts were associated reduced LL/PI mismatch, Figure 1. After propensity matching, expandable grafts remained associated with reduced operative times (Table 2) and accelerated return to work (Figure 2). Static and expandable interbody grafts were associated with significant improvements in pain, physical disability, and quality of life 12 months after surgery, Figure 3.
Conclusion: Both static and expandable inter-body grafts provide significant improvements in one-year pain, disability, and quality of life with similar safety profiles when performing lumbar fusion for stenosis and spondylolisthesis. Expandable interbody grafts may facilitate more efficient graft placement resulting in reduced operative times; and can help achieve more appropriate lumbar lordosis potentially contributing to the accelerated functional recovery and return to work observed here.

227. Titanium Versus Polyetheretherketone (PEEK) Interbody Fusion: Meta-analysis and Review of the Literature

Scott Seaman, MD; Panagiotis Kerezoudis; Mohamad Bydon, MD; James Torner; Patrick W. Hitchon, MD

Introduction: Spinal interbody fusion is commonly performed in the United States[1,2]. Interbody technology includes Titanium (Ti) and Polyetheretherketone (PEEK) cages with distinct biomechanical properties such as elastic modulus, corrosion resistance and promotion of cell adhesion and osseointegration[3-6]. Titanium and PEEK cages have been evaluated in the cervical and lumbar spine, yet conflicting results regarding bone union and interspace collapse rates have been reported[7-9]. We reviewed the available literature evaluating Ti and PEEK cages to assess subsidence and fusion rates.

Methods: Using PRISMA guidelines, we searched Pubmed, Embase, Google Scholar, Cochrane and Scopus databases. Keywords included "titanium" AND "polyetheretherketone", "PEEK" AND "cage", "interbody" AND "cervical fusion", "lumbar fusion" or "interbody fusion". Inclusion criteria were original research articles on human subjects published in peer-reviewed journals. The articles were compared on the basis of design, patient demographics, surgical and radiographic outcomes.

Results: 7 studies were included in the analysis (6 retrospective, 1 randomized controlled trial). There were 4 studies with class of evidence IV, 2 studies with class III, and one study class II. A total of 435 patients (Ti-234, PEEK-201) and 612 levels (Ti-333, PEEK279) were studied. Pooled mean age was 50.8 years in the Ti group and 53.1 years in the PEEK group. Anterior cervical discectomy was performed in four studies (395 levels), transforaminal interbody fusion in two studies (192 levels) and anterior cervical corpectomy and fusion in one study (25 levels). No statistically significant difference was found between groups with fusion (OR 1.16, 95\% C.I $0.59-2.89, \mathrm{p}=0.686$, I-squared $=49.7 \%)$ or subsidence $(\mathrm{OR} 2.74,95 \%$ C.I $0.09-8.28, p=0.073$, I-squared $=62 \%$ ) rates at last follow-up.

Conclusion: Titanium and PEEK cages are associated with similar bone union and subsidence rates. Future prospective randomized controlled trials are needed to further evaluate these materials in surgical and patient reported outcomes following spinal instrumentation.

228. Risk Adjustment Modeling for Urinary Retention in the Michigan Spine Surgery Improvement Collaborative (MSSIC): Results from 8,442 Lumbar Patients

Victor Chang, MD; Michael Bazydlo, MS; Lonni Schultz, PhD; David Nerenz, PhD; Jason M. Schwalb, MD, FACS; Stephen Bartol, MD, MBA, FACS; Muwaffak Abdulhak, MD

Introduction: MSSIC is a prospective registry, and a platform for quality improvement (QI) of elective spine surgery across the State of Michigan. As one of its QI targets, the rate of, and risks for, urinary retention (UR) were assessed. A risk adjustment model was developed to account for differences in patient risks across hospitals for further comparison.

Methods: Using the MSSIC registry, the rate of UR (as defined 
by reinsertion of an indwelling catheter after surgery), and lumbar surgery were performed. Potential risk factors for UR were identified through literature review, bivariate analysis, and consultation with MSSIC surgeons. Odds ratios for each risk factor were estimated with multivariable logistic regression, and were used in generating the expected rate for each hospital. An adjusted rate for any given hospital was calculated by: (Observed rate/Expected rate) * Overall MSSIC rate.

Results: A total of 8,419 patients across 21 hospitals were included in this analysis. The overall rate of UR was $9.2 \%$. Observed rates among hospitals ranged from $1.6 \%$ to $19.3 \%$ (Fig 1). Patients who had UR had a median length of stay (LOS) of 3 days (Interquartile Range: $2-5)(\mathrm{n}=744)$ versus 2 days (IQR: 1-3) for patients without $\mathrm{UR}(\mathrm{n}=$ 7639). Patients with UR also had 1.58 times higher odds of hospital readmission $(\mathrm{p}<0.001)$. The following risk factors were statistically significant: age; male gender; presence of scoliosis; disc herniation; lumbar stenosis; fusion procedure; length of surgery; multi-level procedure (Fig 2). Adjusted rates ranged from 2.3\% to $17.3 \%$ (Fig 3).

Conclusion: UR is a frequent event, and is associated with increased LOS. Within MSSIC there is a wide variability of UR rates between hospitals, with potential for QI initiatives to lower UR and possibly lower LOS across the State of Michigan.

229. Does Saving the Spinous Process in Decompression Surgery Positively Affect Future Instability: A Single Institution Experience

Ziev Moses, MD; Muhammad M. Abd-El-Barr, MD, PhD; Justin Slavin, MD; Viren Sahai Vasudeva, MD; John Chi, MD, MPH; Michael W. Groff, MD, FACS

Introduction: Recent data trends have shown that patients suffering from lumbar spinal stenosis are undergoing higher rates of fusion surgery in situations with and without concomitant spondylolisthesis. Surgeons often perform fusions when they believe a traditional laminectomy would otherwise de-stabilize the target level. We aim to determine whether a bilateral decompression surgery that preserves the midline tension band while decompressing the dural tube has similar clinical outcomes as traditional laminectomy.

Methods: We retrospectively reviewed patients who underwent bilateral decompression surgery (Group $1, \mathrm{n}=33$ ) versus patients who underwent traditional laminectomy (Group 2, n = 40) for lumbar spinal stenosis. All patients underwent a trial of conservative measures prior to surgery as well as pre-operative imaging where the presence of spondylolisthesis was determined. No patients had evidence of a dynamic slip on flexion-extension x-rays. Surgical outcomes were assessed at last follow-up and rated as: excellent, good, fair, or poor. Statistical comparisons between the groups were made with a two-sample unpaired t-test and a Fisher's exact test.

Results: Table 1 compares baseline characteristics of both groups. Notably, the presence and degree of pre-existing slip did not significantly differ between groups. Follow-up averaged 16.6 months. Table 2 describes the clinical outcomes in both groups.

Conclusion: This retrospective series of patients undergoing surgery aimed at maintaining the midline tension band reveals significantly reduced blood loss and shorter hospital stays without significant differences in patient satisfaction compared with traditional laminectomy. While not statistically significant, there were also lower rates of future spondylolisthesis and need for fusion surgery. Further study is warranted with larger numbers of patients to better delineate these potential benefits.
230. Cost-effectiveness of Novel Collagen-mimetic Osteogenic Peptide P15-based Bone Substitute Vs. Local Autograft in Singlelevel Anterior Cervical Discectomy and Fusion (ACDF)

John M. Caridi, MD; Samuel K. Cho, MD; Branko Kopjar, MD

Introduction: Collagen-mimetic osteogenic peptide P15-based graft (i-Factor) is a novel bone substitute that has been FDA-approved for single-level ACDF. We compared cost-effectiveness of P15-based bone substitute and local autograft.

Methods: Two-year horizon was used for the analysis and no discounting applied. Effectiveness estimates were obtained from the RCT IDE study of the P15-based graft. Primary effectiveness endpoint was defined as Overall Patient Success consisting of successful fusion, functional improvement (NDI change $>15$ ), no new neurological deficit and no reoperations or device failures at two years. Direct treatment cost estimates included preoperative workup, anesthesia fee, surgeon fee, surgery and acute care, rehab or home care, medications, and annual monitoring and were converted into 2012 US Dollars.

Results: The probability of Overall Patient Success was $69.8 \%$ for the P15-based substitute vs. $56.4 \%$ for the autograft. The probability of secondary surgery was $7.3 \%$ for the P15-based graft and $10.4 \%$ for the control group. The average treatment cost per patient was $\$ 15,370$ for P15-based substitute and $\$ 15,809$ for local autograft. The cost-effectiveness ratio was $\$ 22,011$ and $\$ 28,056$ per Overall Patient Success for the P15-based graft and local autograft, respectively. The net difference in favor of the novel bone substitute was $\$ 6,045(\mathrm{p}=0.03)$.

Conclusion: Single-level ACDF with the P15-based bone substitute appears to be more cost-effective than with local autograft at two-year follow-up. Longer follow-up is needed to evaluate sustainability of the results.

231. Characterizing Factors Leading To Malpractice Litigation: An Opportunity for Spine Surgeons to Reduce Legal Liability

Nitin Agarwal, MD; Raghav Gupta, BS; Prateek Agarwal, AB; Pravin Matthew; Richard Wolferz, Jr.; Christopher Kim, MD; Arpan V. Prabhu, BS; Adam S. Kanter, MD; David O. Okonkwo, MD, PhD; D. Kojo Hamilton, $M D$

Introduction: Medical malpractice is of substantial interest to the medical community due to concerns costs to the health care system and medical decisions for the sole purpose of reducing legal liability. Physicians in high-risk specialties, such as neurosurgery, are likely to experience malpractice litigation. Thus, this study aimed to characterize the factors leading to malpractice litigation in cases involving spine surgery.

Methods: The Westlaw online legal database (Thomson Reuters, New York, NY) was searched for verdict and settlement reports pertaining to spine surgery from 2010 to 2015. Data was collected regarding type of procedure, patient age and gender, defendant specialty, outcome, award, alleged cause of malpractice, and factors involved in the plaintiff's decision to file. Initial search queried 187 cases whereby exclusion criteria was applied to eliminate duplicates and cases unrelated to spine surgery yielded a total of 98 cases for analysis.

Results: Out of the cases identified and reviewed, the verdict was in favor of the defendant in 62 cases $(63.3 \%)$. Neurosurgeons and orthopedic surgeons were the most common defendants in 29 $(17.3 \%)$ and $40(23.8 \%)$ of the cases, respectively. A perceived lack of informed consent was noted as a factor in $24(24.4 \%)$ of the cases. A failure to diagnose or treat was noted in $31(31.6 \%)$ and $32(32.7 \%)$ cases, respectively. Procedural error was cited in 58 (59.2\%) of cases. Award payouts for plaintiff verdicts and settlements ranged from $\$ 10,000$ to $\$ 16,475,000$, with median payments for plaintiff verdicts being nearly double that of settlements $(\$ 2,525,000$ vs $\$ 1,300,000)$. 
Conclusion: Physicians were not found liable in the majority of cases reviewed. These results suggest that an emphasis on informed consent in spinal surgery is needed, along with close monitoring of patients presenting with related symptoms, and prompt movement towards surgical treatment when indicated.

233. Impact of Resident Participation on Outcomes After Posterior Lumbar Fusion: An Analysis of 5655 Patients from the American College of Surgeons National Surgical Quality Improvement Program Database

Jonathan Tad Yamaguchi, BS; Robert B. Kim; Roxanna Garcia, MD, MS, MPH; Zachary A Smith, MD; Nader S. Dahdaleh, MD

Introduction: Although an integral part of academic medicine, surgical resident participation in the operating room and its impact on patient outcomes have been a topic of debate. No large-scale study has been performed to examine this relationship in posterior lumbar fusion.

Methods: The American College of Surgeons National Surgical Quality Improvement Program (ACS-NSQIP) database was retrospectively reviewed to identify all patients who underwent posterior lumbar fusion (single and multilevel) procedures during 2006-2014. A propensity score-matching algorithm was employed to minimize baseline differences. Multivariate logistic regression analysis of unadjusted and propensity-matched cohorts was performed to examine the effect of resident participation on 30-day postoperative complication rates and length of hospital stay.

Results: A total of 5655 patients met inclusion criteria. The propensity score-matching procedure yielded 1965 pairs of wellmatched nonresident and resident pairs. The multivariate analysis of propensity score-matched population demonstrated that resident involvement in the operating room increased superficial surgical site infections (OR:1.78, $\mathrm{p}=0.037$ ) and thromboembolic complications (OR: $2.02, p=0.023$ ) within 30 -days as well the length of hospital stay (4.0 days vs 4.6 days $\mathrm{p}<0.001)$ and operative duration (198 min vs 243 min, $\mathrm{p}<0.001)$.

Conclusion: This large-scale, population-based study found that surgical resident participation in the operating room during posterior lumbar fusion increased the risk of infectious and thromboembolic complications. This is probably stemming from the increased operative duration.

234. The Timing of Surgery and Symptom Resolution in Patients Undergoing Transforaminal Lumbar Interbody Fusion for Lumbar Degenerative Disc Disease and Radiculopathy

Sharad Rajpal, MD; Alan T. Villavicencio, MD; Sigita Burneikiene, MD; Ewell Lee Nelson, $M D$

Introduction: Success rates of surgical interventions for lumbar disorders vary significantly depending on multiple factors and among them the duration of symptoms. It is not clear whether there is a "cutoff" time when decompression and fusion surgery becomes less effective in the conditions with chronic nerve root compression symptomatology. The main objective of this study was to analyze if the duration of symptoms has any effect on clinical outcomes and primarily resolution of radicular pain symptoms due to degenerative disc disease and stenosis with spondylolisthesis in patients undergoing transforaminal lumbar interbody fusion (TLIF).

Methods: This is a prospective observational study. Eighty-four patients with radicular symptoms due to degenerative disc disease, stenosis or spondylolisthesis with no previous fusion surgeries and undergoing one- to three-level TLIF surgery were enrolled. A comparison between 2 groups of patients was performed to analyze the changes of clinical outcomes for the patients who underwent fusion within $<24$ months vs. $>24$ months.

Results: It was determined that the duration of symptoms was a significant predictor of better leg pain resolution $(\mathrm{p}=0.018)$, but not back pain resolution $(\mathrm{p}=0.27)$, or improvement in ODI $(\mathrm{p}=0.10)$ and SF-36 PCS scores $(p=0.19)$. The patients with shorter duration of symptoms had significantly better radicular symptom resolution $(\mathrm{p}=$ 0.032) compared to patients who waited at least 24 months or longer to undergo fusion.

Conclusion: A shorter duration of symptoms was found to be a statistically significant predictor for better resolution of radicular symptoms in patients undergoing TLIF for painful degenerative disc disease, stenosis, and spondylolisthesis.

\section{Can We Predict Long-term Disability and Discharge Destination After Elective Cervical Spine Surgery?}

Ahilan Sivaganesan, MD; Silky Chotai, MD; Matthew J. McGirt, MD; Clint Devin, $M D$

Introduction: We introduce predictive models for clinically meaningful improvement in disability, as well as discharge destination, after elective cervical spine surgery (CSS).

Methods: 430 patients undergoing CSS were enrolled into a prospective registry. LOESS regression was performed to verify the appropriateness of linear regression. A vast array of patientand diagnosis-related variables were used to power a multivariate regression model for NDI. Possible interactions were also accounted for. We then used Repeated Random Sub-Sampling to validate the performance of our model. A separate logistic regression model was constructed to predict a clinically important improvement in NDI at one year. A third model was similarly developed and validated to predict post-surgery discharge destination (home versus facility).

Results: Our predictive model for 12-month NDI has an R-squared of 0.69 (Figure 1), and in validation, it achieved an R-squared of 0.43 . The predictors, in descending order of influence, are: employment, baseline NDI, diagnosis, smoking, ethnicity, claudication, narcotic use, and symptom duration. Our model for achieving a MCID in NDI has an AUC greater than 0.80 for the development phase and an AUC of 0.65 in validation. The predictors are: baseline NDI, motor deficit, depression, ambulation, revision surgery, employment, diagnosis, smoking, and symptom duration. Finally, our predictive model for discharge destination has an AUC greater than 0.80 for the development phase and an AUC of 0.75 for the validation phase (Figure 2). The predictors are: baseline EQ-5D, number of levels, myelopathy, depression, baseline NDI, and motor deficit.

Conclusion: We present validated models that help predict disability at one year and discharge destination after elective CSS. Our NDI model explains roughly $70 \%$ of the variation in 12 -month disability. Our model for discharge destination has strong predictive accuracy, and can become a useful tool as neurosurgeons seek to better understand patients' post-operative trajectories.

236. Risk Factors for Dysphagia After Anterior Cervical Surgery: Preliminary Results from a Prospective Multicenter Study of the AOSLA Group

Asdrubal Falavigna, PhD; Marco Antonio Koff; Diego Dozza; Juan Pablo Guyot; Ratko Yurac; Pablo Jalón; Jose Maria Jimenez; Barón Záratte; Nicolas Scheverin; Ericson Sfreddo, MD

Introduction: The objective of the study is to determine the degree of dysphagia and predict factors associated with it.

Methods: Prospective and multicenter observational cohorts of patients with spinal degenerative pathologies undergoing anterior 
cervical surgery. The evaluation was made in the preoperative period and on postoperative days: 1, 7,21, and 180. The factors analyzed were related to pathology (type (disc herniation, cervical stenosis, level of the disease, and anterior osteophytes)), patient (ASA score, age, BMI, sex, history of hypertension, diabetes mellitus, pulmonary disease, coronary artery disease, cancer, anxiety, depression, and smoking) and surgery (intubation attempts, right or left-sided approach, release of endotracheal tube cuff pressure, number of fused levels, length of the surgery, and bleeding. The dysphagia was assessed by swallowing satisfaction index and swallowing questionnaires. Patients who refer to having dysphagia after 6 months will undergo an OTL examination.

Results: The preliminary results comprise 85 consecutive patients who underwent anterior cervical approach and 105 consecutive cases in which a posterior lumbar approach was performed. The satisfaction index and the severity of dysphagia (mild, moderate, and severe) were similar between the cervical and lumbar cases at the preoperative evaluation. The patients who underwent cervical approach had a lower satisfaction index on days 1, 7, and 21 after surgery when compared with the lumbar patients. Patients who underwent anterior approach had a major incidence of dysphagia on day $1(49.4 \%$, 42/85). Complaints about swallowing are progressively lower on day $7(25.9 \%, 22 / 85)$ and day $21(11.8 \%, 10 / 85)$. The only variable that showed significance for dysphagia in cervical patients on the day 1 assessment was 2 or more orotracheal intubation attempts $(\mathrm{p}=0.05)$.

Conclusion: The incidence of dysphagia after anterior cervical approach was $49.4 \%, 25.9 \%, 11.8 \%$ and $0.0 \%$ on $1,7,21$, and 180 days after surgery. The severity of dysphagia was usually mild.

\section{The Timing of Venous Thromboembolic Events After Spine Surgery: A Single Center Experience With 6870 Consecutive Patients}

Michael Brendan Cloney, BA; Ekamjeet Dhillon; Helena Roberts; Zachary A. Smith, MD; George R. Cybulski, MD; Tyler R. Koski, MD; Nader S. Dahdaleh, MD

Introduction: Venous thromboembolic events (VTE), including both deep venous thrombosis (DVT) and pulmonary embolism (PE), are a major cause of morbidity after spine surgery. Chemoprophylaxis can prevent VTE. However, the timing of VTE, and the effect of chemoprophylaxis on VTE timing, remains under-investigated.

Methods: We retrospectively examined records from 6870 consecutive spine surgeries at our institution. Data on patient demographics, surgery, hospital course, and timing of VTE were collected. Patients who received chemoprophylaxis were compared to those who did not. Appropriate regression models were used to examine selection for chemoprophylaxis, and the timing of VTE.

Results: Age (OR 1.037 [1.022, 1.051], p < 0.001), longer surgery (OR 1.003 [1.002, 1.004], p < 0.001), history of DVT (OR 1.697 $[1.038,2.776], \mathrm{p}=0.035)$, and fusion surgery $(1.917[1.356,2.709], \mathrm{p}<$ $0.001)$ predicted selection for chemoprophylaxis. Chemoprophylaxis patients experienced more VTE $(3.62 \%$ v. $2.03 \%, \mathrm{p}<0.001)$, but also longer hospital stays (5.0 days v. 1.0 day, HR 0.5107, p < 0.0001) and greater time to occurrence of VTE (median 6.8 days v. 3.6 days, HR $0.6847, \mathrm{p}=0.0003$ ). The cumulative incidence of VTE was lower in the chemoprophylaxis group and correlated with postoperative day for both groups (chemoprophylaxis Spearman $\mathrm{r}=0.9746$ [0.9457, 0.9883], $\mathrm{p}<0.0001$; non-chemoprophylaxis Spearman $\mathrm{r}=$ $0.9061[0.8065,0.9557], \mathrm{p}<0.0001)$. Cumulative VTE incidence and postoperative day are linearly correlated for the first two postoperative weeks (chemoprophylaxis $\mathrm{R}=0.9396, \mathrm{p}<0.0001$; nonchemoprophylaxis $\mathrm{R}=0.8190, \mathrm{p}=0.0003$ ), and for the remainder of the 30-day postoperative period (chemoprophylaxis $\mathrm{R}=0.9535, \mathrm{p}<$ 0.0001; non-chemoprophylaxis $\mathrm{R}=0.6562, \mathrm{p}=0.0058$ ), but the linear relationships differ between these two postoperative periods $(\mathrm{p}<$ 0.0001 for both groups).

Conclusion: Anticoagulation reduces the incidence of VTE events after spine surgery. The cumulative incidence of VTE rises steadily within the first two postoperative weeks.

\section{Bone Morphogenetic Protein (BMP) and Surgical Site Infection Rates Following Lumbar Spine Surgery: A Report From Washington State SCOAP-CERTAIN Collaborative}

Vijay Yanamadala, MD; Jean-Christophe A. Leveque, MD; Sara Khor; Quinlan Buchlak; Amy Cizik; Rajiv Sethi, MD

Introduction: Bone morphogenetic protein (BMP) increases fusion rates and decreased long term reoperation rates. However, there is concern that BMP may increase surgical site infection (SSI) and wound complications. The Washington State Surgical Care and Outcomes Program (SCOAP) is a large multi-institutional prospective database that is collected at 20 hospitals in Washington. We queried the SCOAP database to examine the correlation between BMP usage and SSI.

Methods: A prospective cohort undergoing lumbar spine fusion in SCOAP were stratified according to the use of BMP during surgery. We assessed patient demographics including co-morbidities such as diabetes, hypertension, and active smoking status, and diagnosis to ascertain any confounding parameters. SSI rates were determined over a 90-day period and compared in cohorts who underwent fusions with or without BMP after adjustment. Data were analyzed using a random effects logistic model (index), and a time-to-event analysis (follow-up) using Cox proportional hazards.

Results: 2,324 patients underwent first-time open lumbar fusion across 20 hospitals (510 with BMP; 1814 without BMP). Onehundred $(4.3 \%)$ patients developed SSI within 90 days. The rate of SSI in patients who did and did not receive BMP was $3.5 \%$ and $4.5 \%$, respectively $(\mathrm{p}>.05)$. In our univariate analysis, a higher proportion of the surgical procedures involving BMP were performed by neurosurgeons vs. orthopedic surgeons. BMP procedures were more often conducted in large hospitals and academic hospitals. After adjustment for patient, operative, and hospital characteristics in the random effects model, BMP was associated with a no statistically significant change in SSI (OR 0.66, 95\% CI: 0.36, 1.20).

Conclusion: BMP use does not alter the rate of perioperative SSI, despite the higher propensity of surgeons to use BMP in patients with known risk factors for SSI, including active smokers and patients on steroids. These data suggest that BMP can be used without concern for increasing local wound complications.

239. Pre-Clinical and Initial Clinical Experience of a Novel Rapid Intra-Operative Registration Technique of Optical MachineVision to Pre-Operative Imaging for Spinal Surgical Navigation

Raphael Jakubovic, MSc; Daipayan Guha, MD; Michael Lu; Shaurya Gupta; David W. Cadotte, MSc, MD; Chris Heyn, MD, PhD; Peter Howard, MD; Todd Graham Mainprize, MD; Albert Yee, MD; Victor Xiao Dong Yang, $M D, P h D, M S c, B S c$

Introduction: Computer-assisted navigation is standard for most cranial procedures, and is employed in the spine to guide instrumentation, bony decompression and soft-tissue resection. Current navigation techniques register patient surface anatomy to either pre- or intra-operative imaging. The former is hampered by lengthy setup and registration protocols as well as an inability to account for intra-operative tissue movement; the latter requires setup of cumbersome imaging units, and exposes patients to additional intra-operative radiation.

Methods: A novel structured-light-based optical topographic imaging (OTI) system was developed for spinal neuronavigation (Fig. 1). Initial validation was performed in 4 adult swine, followed 
by clinical validation in 53 patients undergoing open posterior thoracolumbar instrumentation. Registrations to thin-slice preoperative CT imaging were performed using OTI and benchmarked to existing neuronavigation systems (StealthStation/O-Arm, Nav3i). Navigation data was compared to post-operative imaging, and the absolute deviation of final screw positions from intra-operativelyplanned trajectories computed (Fig. 2). Final screw positions were also graded clinicoradiographically using the Heary classification, independently by 7 raters.

Results: For 71 screws placed in 4 adult swine, translational and angular median $(95 \%)$ errors were $1.67 \mathrm{~mm}(5.12 \mathrm{~mm}) / 4.37^{\circ}\left(12.95^{\circ}\right)$ and $1.63 \mathrm{~mm}(7.81 \mathrm{~mm}) / 6.50^{\circ}\left(17.76^{\circ}\right)$ in the axial and sagittal planes, respectively. In human clinical trials, with 129 screws placed with OTI in 22 patients and 209 screws placed with benchmark systems in 31 patients, OTI registration was achieved using 2251+/-1047 surface points per vertebral level (mean+/-SD). Registration and manual verification of navigation accuracy were performed significantly faster than both benchmark systems (mean 40s vs. 258s and 794s). Translational and angular median $(95 \%)$ errors of $1.21 \mathrm{~mm}(2.99 \mathrm{~mm}) / 1.80^{\circ}\left(6.60^{\circ}\right)$ and $1.45 \mathrm{~mm}(3.57 \mathrm{~mm}) / 2.02^{\circ}\left(7.62^{\circ}\right)$ in the axial and sagittal planes, respectively, were achieved with OTI. No significant differences in clinicoradiographic grading or absolute quantitative errors between OTI and benchmark systems were observed.

Conclusion: Optical machine vision is faster and comparably accurate for spinal neuronavigation. Rapid structured-lightillumination allows efficient initial and repeat registrations with minimal workflow disruption.

\section{Dysphagia in Standalone Versus Conventional Anterior Cervical Discectomy}

Christian Fisahn, MD; Fernando Enrique Alonso, MD; Marc Daniel Moisi, MD; Rod J. Oskouian, MD; R. Shane Tubbs, PhD, PA-C; Jens Chapman, $M D$

Introduction: Dysphagia is an extensively described complication of anterior cervical discectomy and fusion. It has been proposed that mechanical irritation, additional dissection or displacement of the esophagus by plate placement may contribute to a greater incidence of post-operative dysphagia. The aim of this study was to compare dysphagia symptoms and pain severity of standalone cage systems versus interbody devices in combination with an anterior plate.

Methods: A retrospective cohort study identified 377 consecutive patients (standalone, $\mathrm{n}=211$; plate and cage, $\mathrm{n}=166$ ) meeting the study criteria between the years 2014 to 2015. Patient-specific characteristics and surgical characteristics and Numeric Pain Rating Scale (NRS) scores were collected preoperatively. Complication and readmission rates, the Dysphagia Disability Index (DDI), and NRS scores were collected at one year and two years post-operatively.

Results: Both groups were similar with respect several comorbidities including the Charlson score, and pre-operative NRS scores $(5.3 \pm 2.1$ versus $5.4 \pm 1.9$ in stand alone and plate and cage groups). The stand along group experienced a higher rate of symptomatic non-union with reoperation but this did not achieve statistical significance ( $9 \%$ versus $4 \% ; p=.07)$. There was a significantly greater improvement in neck pain scores in the plate and cage group after one and two years post-surgery compared to the stand alone group (2.3 and 3.1 versus 1.6 and 1.5 , respectively; $\mathrm{p}<.01$ ). Post operative DDI scores were similar at 2 years post-surgery $(9.4 \pm$ 11.9 versus $9.5 \pm 11.7$ ).

Conclusion: Chronic dysphagia scores are comparable between standalone cage systems and interbody grafts with plating. We observed a greater improvement in neck pain and a lower incidence of nonunion in the group that underwent interbody graft and plate placement at followup. Further research is needed in order to determine factors that may lead to a higher rate of non union for patients undergoing stand alone cage placement.

\section{Use of Liberal Hemoglobin Triggers in Spine Surgery}

Taylor Elise Purvis, BA; C. Rory Goodwin, MD, PhD; Rafael De la Garza-Ramos, BA; A. Karim Ahmed; Virginie Lafage, PhD; Brian J. Neuman, MD; Peter G. Passias, MD; Khaled Kebaish, MD; Steven M. Frank, MD; Daniel M. Sciubba, MD

Introduction: While blood transfusions are commonly used in spine surgery, they are associated with increased intra- and postoperative patient morbidity. No studies have examined the association between the reliance on liberal hemoglobin triggers that is, an intraoperative hemoglobin level of $10 \mathrm{~g} / \mathrm{dL}$ or greater, or a postoperative level of $8 \mathrm{~g} / \mathrm{dL}$ or greater and perioperative morbidity in spine surgery patients. In this study, we examined the association between perioperative outcomes and a liberal transfusion strategy during spine surgery.

Methods: Institutional inpatient surgical records were queried for spine surgeries between 2008 and 2015. Included in this group were patients undergoing spinal fusion, tumor-related surgeries, and other spine surgeries. Variables analyzed included mortality, in-hospital morbidity, and length of stay associated with liberal transfusion triggers.

Results: A total of 6931 patients undergoing spine surgery were identified and separated into eight major procedural groups. For patients with an entire hospital stay hemoglobin nadir between 8 to $10 \mathrm{~g} / \mathrm{dL}$, transfused patients had longer in-hospital stays (median [IQR], 6 [5-9] vs. 4 [3-6] days; $\mathrm{P}<0.0001)$ and higher perioperative morbidity $(\mathrm{n}=145,[11.5 \%]$ vs. $\mathrm{n}=74,[6.1 \%] ; \mathrm{P}<0.0001)$ than those not transfused. Moreover, even after adjusting for potential confounders such as age, estimated blood loss, number of operated levels, ASA class, CCI score, and surgery type, patients with a hemoglobin nadir of $8-10 \mathrm{~g} / \mathrm{dL}$ who were transfused had an independently higher risk of perioperative morbidity (odds ratio $[\mathrm{OR}]=2.12 ; 95 \%$ confidence interval [CI], 1.24-3.64; $\mathrm{P}=0.006$ ).

Conclusion: Reliance on liberal transfusion triggers is associated with worse perioperative outcomes, even after adjusting for confounders. Clinicians may wish to consider alternative transfusion triggers for spine surgery patients to reduce patient morbidity.

\section{The Effect of Time to Surgery for Degenerative Lumbar Stenosis in a Workers' Compensation Setting}

Erik Y. Tye, BA; Joshua T. Anderson, MD; Jay M. Levin, BA; Arnold Haas; Stephen T. Woods; Nicholas U. Ahn, MD

Introduction: The effect of time to surgery for the indication of degenerative spinal stenosis (DLS) is poorly characterized. The goal of the study was to determine if outcomes of surgery for DLS in a Workers' Compensation population are affected by time to surgical decompression.

Methods: 227 Ohio WC subjects were identified who underwent primary lumbar decompression for the indication of LSS. We allocated patients into two groups: those that received operative decompression before and after 1 year of onset of symptoms. Our primary outcome was if patients were able to make a stable return to work (RTW). The authors classified subjects as RTW if they returned within 2 years after surgery and remained working for more than 6 months. We measured a number of secondary outcomes.

Results: The early cohort had a RTW rate of $50 \%(25 / 50)$, which was significantly higher than the late cohort in which only $30 \%$ $(53 / 117)$ of subjects met our RTW criteria $(p=0.01)$. A logistic regression was performed to identify independent variables that predicted RTW status. After correcting for various factors, our model showed that time to surgery remained a significant negative predictor of RTW status $(p=0.04)$. Compared with the late surgery group, patients who had a decompression before 1 year cost on average, $\$ 37,332$ less in total medical costs that those who opted 
for surgery after 1 year $(\mathrm{p}=0.01)$. Furthermore, total medical costs accrued over 3 years after index surgery was on average, \$13,299 less when patients received their operation within 1 year after onset of $\operatorname{symptoms}(\mathrm{p}=0.01)$.

Conclusion: The findings in the present study demonstrate that time to surgery has a significant impact on return to work rates on WC subjects receiving lumbar decompression for LSS. The promising results presented here can perhaps be used to guide surgical decisionmaking and provide predictive value for the WC population.

243. Lumbar Fusion for Symptomatic Mechanical Disc Collapse Without Stenosis Improves Pain, Disability, and Quality of Life: Rethinking the Debate on Degenerative Disc Disease (DDD)

Matthew J. McGirt, MD; Clinton J. Devin, MD; Deborah Pfortmiller; Silky Chotai, MD; Mohamad Bydon, MD; Steven D. Glassman; Anthony L. Asher, MD, FACS

Introduction: The efficacy of lumbar fusion for degenerative disc disease (DDD) without stenosis remains unclear and highly debated by physicians, payers, and policy makers. Many patients with medically refractory mechanical axial back pain and significant lumbar disc height collapse are denied access to fusion procedures by their respective payer policies. Utilizing a standardized definition for DDD, we set out to determine if lumbar fusion was associated with improved patient-reported health benefits in a prospective nationwide registry.

Methods: N2QOD registry collects one-year patient-reported outcomes(PROs) after surgical care for six lumbar diagnosis groups. We queried all surgical cases that were enrolled under the DDD diagnosis group "symptomatic mechanical disc collapse" defined a priori as reproducible mechanical axial back pain with $>50 \%$ disc height loss without stenosis or listhesis proven to be medically refractory $>3$ months. The relative prevalence, safety, and effectiveness of surgery for symptomatic mechanical disc collapse was assessed.

Results: 7618 patients from 74 hospitals in 26 U.S. states were enrolled and completed 12month PROs. Symptomatic mechanical disc collapse represented the minority of these surgical cases at N2QOD hospitals [42(0.6\%)],Table 1. All cases underwent single-level fusion and associated with mean $\mathrm{EBL}(327 \mathrm{cc})$, length of stay(3.5days), discharge to inpatient rehab(12\%), 30-day morbidity $(9 \%)$ and 90 -day all-cause readmission( $9 \%$,Table 2. Lumbar fusion resulted in 12 month improvement in back pain(BP-NRS: 8 vs 5.3,p $<0.001$ ); leg pain(LP-NRS:6.5 vs 4.3,p<0.001); disability(ODI:54 vs 42,p<0.001); and quality of life(EQ5D:0.49 vs 0.59,p $<0.005$,Figure 1. Postoperative return to work reached $66 \%$ at $3 \mathrm{mo}$ and $100 \%$ by $12 \mathrm{mo}$,Figure 2 .

Conclusion: Lumbar fusion for symptomatic mechanical disc collapse without stenosis or listhesis represented the minority of surgical practice at the 74 N2QOD centers between 2010 and 2015. Those patients undergoing lumbar fusion for this narrowed definition of DDD experienced improvements in all measured domains of health, suggesting lumbar fusion is an effective treatment for back pain arising from mechanical disc collapse

244. Comparative Study on the Zero-Profile Cage with Screws System and Stand-alone Rectangular Peek Cage Systemaugmented Fusions After Single Level Anterior Cervical Discectomy

\section{Jong Tai Kim; Doo Yong Choi; Myeng Hoon Shin; Ho jin Lee}

Introduction: A Zero-profile Implant for Anterior Cervical Interbody Fusion is known as implant which may provide strong fixation and reduce the complications associated with other high profile cervical fixation systems. But, there is a question about role of screws at the zero-profile implant for the immediate strong fixation and improvement of fusion late compared with stand-alone PEEK cage. So, we evaluated and compared radiological results, clinical outcomes after the Zero-Profile cage with screws system and standalone rectangular peek cage system-augmented fusions in patients undergoing single level anterior cervical discectomy and fusion (ACDF).

Methods: The two groups compared were: 34cases of anterior interbody fusion with zero-profile cage with screws system (group A) ; 30 cases of anterior interbody fusion with stand-alone rectangular PEEK cage system (group B). Mean follow-up period were 36.6 months in group A and 37.5. months in group B. All patients could be followed-up for at least 12 months. Radiological (construct height and height loss which means disc height loss, segmental angle, cervical lordotic angle, especially, subsidence rate) and clinical assessments were performed preoperatively, immediately postoperatively, and at $1,3,6$ months and final follow up postoperatively.

Results: The mean construct heights (distance between upper endplate of upper body and lower endplate of lower body of the fused segment) were $30.1 \mathrm{~mm}$ preoperatively, $34.5 \mathrm{~mm}$ at 1 day, $33.3 \mathrm{~mm}$ at 1 months, $33.1 \mathrm{~mm}$ at 6 months and $32.5 \mathrm{~mm}$ at final follow-up after surgery in group A, and 34.0, 36.9, 35.8, 34.6 and 33.4 in group B, respectively. In group B, early, progressive and significant disc height loss and delayed fusion processing were observed during postoperative follow- up period. Subsidence (defined as any settlement in disc height of at least $3 \mathrm{~mm}$ on lateral radiographs) rate were $11.7 \%$ (4/34patients) in group A, 26.7\% (8/30 patients) in Group B. Clinical results according to the Odom's criteria were excellent and good in $88.2 \%$ ( group A ), $76.7 \%$ ( group B ). There were no significant complications associated with both systems except a case of minor $(2.5 \mathrm{~mm})$ pull-out of left lower screw of zero-profile system.

Conclusion: In this comparative study, the authors demonstrated that implantation of the zero-profile cage with screw systems maybe have the advantages of prevention of significant undesirable subsidence \& related complications and ameliorate the clinical outcome compared to implantation of the stand-alone rectangular cage.

\section{The Relationship Between the Deep Paraspinal Muscles of the Cervical Spine and Fusion Rate After Anterior Decompression and Fusion}

\section{Sung Bum Kim; Man-Kyu Choi}

Introduction: Deep paraspinal muscles (DPM) in the cervical spine function to preserve spinal stability and mobility. However, compared with the other factors affecting bone union after spinal surgery, there are no studies evaluating the relationship between DPM and fusion rate. To evaluate the relation of the cross-sectional area of DPM and the fusion rate after anterior cervical decompression and fusion (ACDF) using cage alone.

Methods: A total of 134 adult patients with degenerative cervical disease who underwent ACDF using cage alone at C5-C6 or C6-C7 segments were included in this study. The muscle cross-sectional area (MCSA) of the longus colli (LCo), longus capitis (LCa), semispinalis cervicis (SSC), and multifidus (MF) were measured using preoperative cervical magnetic resonance imaging at three segments, and the bone union was evaluated using dynamic cervical X-rays.

Results: The differences in the extensor (SSC, MF) MCSA at most of segments were significant between the union and non-union groups (except $\mathrm{C} 7-\mathrm{T} 1$ segment, all $\mathrm{p}<0.05$ ). On multivariate analysis, the MCSAs of extensor at C5-C6, C6-C7 were an independent factor in decreasing the possibility of non-union $(\mathrm{OR}=0.741,0.803$, all $\mathrm{p}<0.05)$. Pearson analysis demonstrated that the extensor MCSA had significant negative correlation with the fusion time at $\mathrm{C} 4-\mathrm{C} 5$ $(\mathrm{r}=-0.410, \mathrm{p}<0.05), \mathrm{C} 6-\mathrm{C} 7(\mathrm{r}=-0.479, \mathrm{p}<0.05)$ segments in $\mathrm{ACDF}$ 
at $\mathrm{C} 5-\mathrm{C} 6$ and all $(\mathrm{r}=-0.473,-0.518,-0.612$, all $\mathrm{p}<0.05)$ segments in $\mathrm{ACDF}$ at $\mathrm{C6}$-C7.

Conclusion: The MCSAs of extensor of union group were significantly larger than non-union group's one. Moreover, as the extensor MCSA was larger, the fusion time was short.

246. Vertebral Segmentation Osteotomy (type 2.5 osteotomy) for Degenerative Lumbar Sagittal Imbalance: Technical Motes and Surgical Results

Gwang Soo Lee: Dal-Dung Ryu; Kyung Hyun Kim; Jeong-Yoon Park; Sung-Uk Kun; Dong-Kyu Chin; Deun-Su Kim; Yong-Eun Cho

Introduction: Perioperative complication and reoperation rate due to high intraoperative blood loss, pseudoarthrosis and mechanical failure after pedicle subtraction osteotomy is significantly higher especially in patients with degenerative sagittal imbalance. Type II osteotomy as known as Smith Peterson Osteotomy is useful surgical techniques for correction of degenerative lumbar sagittal imbalance. However only posterior resection could not sufficiently release vertebral segment amd cause increased bone-metal interface stress, thus, recently we modified type II osteotomy with extended discectomy to make each vertebral segment fully mobile. Thus we described surgical techniques and early perioperative results.

Methods: A total of 17 patients underwent surgical corrective surgery using vertebral segmentation osteotomy for degenerative sagittal imbalance. We described detailed surgical techniques and limitations for correction. Postoperative clinical results includes SF-36, ODI, VAS and radiological results and complications are presented.

Results: Mean osteomized segments per patients were 2.75 (range, 2-3). Mean correction angle was 16.77(8.7-35.2). All postoperative sagittal parameters such as sagittal vertical axis, pelvic tilt, and PI-LL showed significant improvement compared to preoperative data and properly was corrected within normative data according to previous research. Radiological restoration was insufficient especially in patients with male, rigid spinal deformity. Preoperative range of motion, disc space preservation, and vacuum disc existence was important factor for sufficient correction.

Conclusion: Degenerative sagittal imbalances were corrected successfully using vertebral segmentation osteotomy without early perioperative complication. This surgical techniques could be viable option for treating the patients with degenerative sagittal imbalance. Preoperative meticulous patients selection for successful correction using this technique is important. Intraoperative epidural bleeding control, careful manipulation of root ganglion, and wide range of discectomy through the lateral and anterior margin are important key procedure for this technique.

\section{What is the Proper Management for Pyogenic Spondylodiscitis? Conservative? Surgery?}

James Ki Shinn; Seung Hwan Yoon; DoKeun Kim; Ji Yong Kim

Introduction: With population has been aging, infectious spondylodiscitis is being diagnosed with increasing frequency due to the increasing numbers of elderly patients, use of invasive spinal procedures. We discuss our experience on the management of infectious spondylodiscitis.

Methods: We reviewed the management, radiologic and clinical outcomes of 73 of pyogenic spondylitis patients treated between January 2008 and December 2012, and minimum follow up periods were 2 years. Patients were divided by conservative (group A) or surgical (group B) management. Surgery group and conservative treatment group in pyogenic spon-dylodiscitis were compared according to the clinical and radiologic profiles. Radiologic evaluation was assessed and clinical outcome was evaluated with Visual Analogue Scale (VAS) for pain and Oswestry Disability Index (ODI) for disability. Also, hospital stay term and medical cost were included in this study.

Results: Mean age of patients was 67.1 of group A and 65.7 of group B, respectively. For early surgical management, 42 out of 73 patients $(57.6 \%)$ were treated. Mean hospital stay was $32.7 \pm 4.6$ days for group A and $21.3 \pm 3.7$ days for group B, respectively. Medical costs of group B $(2,235,000$ Korean Won) was not more than that of group A (2,380,000 Korean Won). Radiologic outcome that of sagittal balance, local angle was different between two groups. Clinical outcomes including VAS and ODI were also different between two groups.

Conclusion: Surgical management such as debridement, decompression, restoration of spinal alignment, and correction of instability is considered for treatment options for failure of conservative therapy or severe pyogenic spondylodiscitis.

\section{Clinical Outcomes of Correcting Cervical Deformity in Cerebral Palsy Patients}

Yoon Ha MD, PhD; Chang-Kyu Lee; Dong Ah Shin; Seong Yi, MD, PhD; Keung Nyun Kim, MD, PhD; Hyun Chul Shin, MD; Do-Heum Yoon, $M D$

Introduction: To assess several different cervical alignment parameters to determine the clinical relationship between cerebral palsy (CP) with cervical spondylotic myelopathy (CSM) and cervical deformity.

Methods: This study included consecutive patients $(n=31)$ with CP-CSM who underwent cervical operation between January 2006 and January 2014 and who had cervical deformities such as angular and translational deformities. Cervical spine alignment was assessed with the following parameters: the $\mathrm{C} 2-7 \mathrm{Cobb}$ angle, $\mathrm{C} 2-7$ sagittal vertical axis (SVA), and T1 slope minus C2-7 Cobb angle. Other clinical values were the manual muscle test, spasticity, grip and pinch test, box and block test, and Jebsen-Taylor hand function test. Outcome assessments (Oswestry Neck Disability Index [NDI] and modified Barthel Index [MBI]) were obtained for all patients pre- and postoperatively.

Results: Mean follow-up duration was 3.5 years. There were 13 patients in the corrected group and 18 in the not corrected group. Angular and translational correction were $19.0^{\circ}$ (C2-7 Cobb angle), $19.8^{\circ}$ (T1 slope- C2-7 Cobb angle), and $16 \mathrm{~mm}$ (C2-7 SVA). Postoperative NDI showed greater improvement in the corrected group than the uncorrected group ( $\mathrm{p}=0.049)$. In the corrected group, grip power increased postoperatively $(8.9 \pm 8.9$ vs. $15.5 \pm 8.3$; $p=$ $0.021)$.

Conclusion: Surgical treatment for patients with CP-CSM deformity helped alleviate symptoms. Postoperative NDI scores and hand function improved in patients with CP-CSM deformity, especially those in the corrected group. Clinicians should consider correcting the deformity in patients with CP-CSM

249. Effects of Epidural Adhesion in Herniated Lumbar Disc: a 2D Non-linear Finite Element Analysis

Nam Lee; Dong Ah Shin; Gyu Yeul Ji, MD; Seong Yi, MD, PhD; Do Heum Yoon; Keung Nyun Kim, MD, PhD; Yoon Ha, MD, PhD

Introduction: It is well documented that epidural adhesion is associated with spinal pain. However, the underlying mechanism of spinal pain generation by epidural adhesion has not yet been elucidated. 
Methods: A two-dimensional nonlinear FE model of the lumbar spine was developed, consisting of the intervertebral disc, dura, spinal nerve, and lamina. The annulus fibrosus and nuleus pulpous were modeled as hyper elastic using Mooney Rivlin equation. The FE mesh was generated and analyzed by Abaqus (3DS, RI, United States).Epidural adhesion was simulated as assigning rough contact, in which no slip occurs once two surfaces are in contact, between the dura and posterior annulus fibrosus

Results: The FE model of adhesion showed significant stress concentration in the spinal nerve, especially on the dorsal root ganglion (DRG). The stress concentration was caused by the lack of adaptive displacement between the dura and posterior annulus fibrosus. Without adhesion, adaptive displacement was observed with decreasing stress in the spinal nerve and DRG.

Conclusion: The current study clearly demonstrated that epidural adhesion provokes significant increase of stress in the spinal nerve, especially the DRG. We believe that the increased stress on the spinal nerve can elicit more severe spinal pain under similar magnitude of lumbar disc protrusion.

\section{The Correlation Between Definite Preoperative Differential Diagnosis and Pathologic Results of Nerve Biopsy}

Linden Fornoff, MD; Kyle Schmidt; Steven Tenny; Kenneth A. Follett, $M D$

Introduction: Nerve biopsies are an important aspect of confirming a diagnosis for a number of neuropathies. The specimen, however, can frequently yield the irritating diagnosis of "nondiagnostic." Our aim is to determine if there is correlation between the requesting provider having a narrow differential diagnosis with the attainment of diagnostic pathologic specimen(s).

Methods: A retrospective review of a single institution's nerve biopsies was performed. 76 nerve biopsies were completed at the University of Nebraska Medical Center from January 1, 2012, to July 1, 2016. Information regarding pre-operative differential diagnoses, pathologic results, and treatment alteration secondary to pathologic diagnosis was recorded. A Fisher exact test was used to determine the $p$-values of three different observations: definite pre-operative differential diagnoses and definite pathologic diagnoses, definite preoperative differential diagnoses and alteration of treatment, and lastly, definite pathologic diagnoses and alteration of treatment.

Results: Of the three aforementioned observations, the last one, definite pathologic diagnoses and alteration of treatment, was statistically significant with a $p$-value $=0.0001$. The remaining two observations: definite pre-operative differential diagnoses and alteration of treatment revealed a $\mathrm{p}$-value $=0.1487$, and definite preoperative differential diagnoses and definite pathologic diagnoses had a $p$-value $=0.0868$. These neared statistical significance and were likely underpowered $(\mathrm{n}=76)$ for this study.

Conclusion: In conclusion, if a clinician has a definite pathologic diagnosis after requesting a nerve biopsy, then the biopsy will be more likely to alter that patient's treatment plan. One may also surmise that the clinician with a defined list of differential diagnoses will be more likely to attain a definite pathologic diagnosis from that nerve biopsy, as this neared statistical significance, but was likely underpowered.

\section{Neurologic Outcomes Following Proximal Hamstring Avulsion and Repair}

\section{Thomas J. Wilson, MD; Aaron Krych, MD; Robert J. Spinner, MD}

Introduction: Muscle bellies of the hamstring muscles are intimately associated with the sciatic nerve, putting the sciatic nerve at risk of injury associated with proximal hamstring avulsion. There are few data informing the magnitude of this risk, identifying risk factors for neurologic injury, or determining neurologic outcomes in patients with distal sciatic symptoms following surgery. The goal of the current study was to characterize the frequency and nature of sciatic nerve injury and distal sciatic nerve-related symptoms following proximal hamstring avulsion and to characterize the neurologic outcomes following surgical repair.

Methods: Retrospective study of patients with proximal partial or complete hamstring avulsion. The outcome of interest was neurologic symptoms referable to the sciatic nerve distribution below the knee. For operative patients, neurologic symptoms were compared pre- and post-operatively. Standard statistical methods were used.

Results: The cohort consisted of 162 patients; 67 operative (41.4\%) and 95 non-operative (58.6\%). Sciatic nerve related symptoms were present in $45(27.8 \%)$ patients; $8(4.9 \%)$ motor deficits, $11(6.8 \%)$ sensory deficits, and $36(22.2 \%)$ with neuropathic pain. Among the operative cohort, $3 / 3(100.0 \%)$ patients showed improvement in their motor deficit post-operatively, $3 / 4(75.0 \%)$ sensory symptoms improved, and $17 / 19(89.5 \%)$ had improvement of pain. A new or worsening deficit occurred in $5(7.5 \%)$ patients post-operatively; 2 (3.1\%) motor deficits, 1 (1.5\%) sensory deficit, and $3(4.5 \%)$ with new pain. Predictors of operative intervention included lower age $(\mathrm{p}$ $=0.001)$ and complete avulsion $(p<0.001)$. Presence of neurologic deficits was not predictive.

Conclusion: Sciatic nerve-related symptoms following proximal hamstring avulsion are under-recognized. Currently, neurologic symptoms are not considered when determining whether to pursue operative intervention. Given the high likelihood of improvement post-operatively, neurologic symptoms should be discussed in the context of the neurologic risk reported here when discussing operative intervention.

\section{Outcome Analysis of Common Peroneal Nerve Neuroplasty at the Lateral Fibular Head}

\section{John Souter; Kevin Swong, MD; Vikram C. Prabhu, MD, MS}

Introduction: Common Peroneal Nerve (CPN) neuropathy is the most common lower extremity entrapment neuropathy. Patients present with painless weakness. Diagnosis is confirmed by physical examination, and may be aided electrodiagnostic studies. We examined our patient experience to see if BMI or time to surgery were significant predictors of outcome.

Methods: IRB approval was obtained through our hospitals administration. This was a retrospective review of a prospectively maintained databse. We examined age, gender, body-mass index (BMI), time to surgery, operative time, and surgical costs.

Results: 35 patients underwent CPN neuroplasty over a 10 year period. 22 were male and 13 female, and average age was 47 years. All had a loss of sensation in the distribution of the CPN, 18 patients reported pain, and 28 had weakness. Predisposing trauma was reported in 13 patients, and 13 patients had a previous surgical procedure. The average time between onset of symptoms and surgery was 114 weeks. A minimum of 3-month follow up was available in 30 patients; average follow up was 54 weeks. Of the 25 patients who presented with abnormal lower extremity motor scores and had a 3-month follow up, 22 had at least 1 grade improvement in motor scores, 2 patients experienced no change, and 1 patient experienced slightly worse motor function. 28 patients reported improved sensation, while 2 patients reported worsened sensation. Patients with an increased BMI and increased time to surgery had a non-significant trend towards worse outcomes.

Conclusion: Surgical neuroplasty of the CPN at the lateral fibular neck is an effective and safe procedure. Even though there was a trend worse outcomes with increasing BMI and time to surgery, there was no significant difference based on BMI or time to surgery. Taken together, these results lend credence to common peroneal nerve 
decompression regardless of the patients BMI or possible delay to surgery.

\section{Osteopontin Enhances IGF-1 Signaling in Promoting Axon Regeneration After Nerve Injury in the Central Nervous System}

\section{Yi Lu MD, PhD; Hong Guo}

Introduction: Spinal cord injury $(\mathrm{SCI})$ often results in permanent deficits below the level of the injury. Adult mammalian central nervous system (CNS) has limited inherent capacity to regenerate and restore functional connectivity across the injury site. During development, Insulin-like growth factor-1 (IGF-I) specifically enhances central nervous system axon outgrowth. Corticospinal motor neurons express high levels of IGF-1 receptors (IGF-1R) during development. The enhancement by IGF-I is mediated via the IGF-IR and its downstream PI3-kinase/Akt signaling pathway. In mature CNS, IGF-1R was downregulated on the CNS axons and leads to the the inability of IGF-1 to elicit regeneration from the mature axons. Through a screening experiment, we identified osteopontin (OPN), a secreted, glycosylated phosphoprotein, enhances the IGF-1 function and promotes mature neurons axon regeneration combined with IGF-1.

Methods: We used cell culture system to study the molecular signal pathway of the synergistic effect of OPN with IGF-1. We used primary cortical neuron cell culture system to study the neurite outgrowth with OPN and its different segments. We also set out to study the mechanisms how OPN promotes IGF-1 signaling in mature CNS system.

Results: Using the primary cortical neuron cell culture system, we demonstrated that OPN plus IGF-1, but neither OPN or IGF-1 alone, promotes IGF-1R phosphorylation (activated form of IGF-1R) and the activation of its downstream PI-3K pathway. Phosphorylation of the $\beta$-subunit of IGF-1R is a critical step to inducing neurite outgrowth by IGF-1. In the primary neuron cell culture system, we demonstrated that OPN or its N-terminal segment, but not the C-terminal segment, promotes significant neurite outgrowth. We also identified that OPN promotes IGF-1R distributing into distinct cholesterol-rich microdomain of the cell membrane components (lipid raft) and the aggregation of IGF-1R into the lipid raft promotes the phosphorylation of IGF-1R. This process is likely mediated with $\beta 1$ integrin, as the deletion of the integrin binding site on the OPN abolish the effect of OPN in promoting IGF-1/IGF-1R signaling.

Conclusion: OPN promotes IGF-1/IGF-1R signaling in axon regeneration response in adult central nervous system.

\section{Intra-operative Localization of Peripheral Nerves Using Ultrasound-guided Instrument Placement in Patients with Peripheral Nerve Pain Syndromes}

P. Troy Henning, DO; Thomas J. Wilson, MD; Matthew Willsey, MD; Jessin Koshy John, MD; Miriana Popadich, MSN; Lynda Jun-San Yang, $M D, P h D$

Introduction: Nerve transections in patients with peripheral nerve pain have traditionally relied on surgical landmarks. However, this can be challenging, especially for patients with variable nerve course, elevated body-mass index, and in re-do cases. We propose a novel technique for nerve localization using ultrasound guided (USG) instrument placement.

Methods: Three patients with mononeuropathies (2 meralgia paresthetica and 1 saphenous neuropathy) were selected for nerve transection. Intra-operatively, under direct USG, pituitary forceps were clamped on the target nerve through a small skin incision. Through a separate incision, dissection was carried down to a sub- fascial layer where the forceps delivered the nerve into view for transection. Operative time, incision length, and body-mass index (BMI) were recorded for each case.

Results: The time from the start of ultrasound visualization to nerve transection varied from 7-14 minutes for lateral femoral cutaneous nerve and was 10 minutes for saphenous nerve transection. The average operative time (OT), from incision to closure, for all cases was $19 \mathrm{~min}$. This is in contrast to traditional open cases with average time of approximately 150 minutes. With an average cost of $\$ 92 /$ minute OT, this equates to $\$ 12,052$ in potential cost savings per case. The average primary incision length was $29 \mathrm{~mm}$. BMI for the 3 patients were $29.1,30.2$, and $38.7 \mathrm{~kg} / \mathrm{m} 2$.

Conclusion: While ultrasound assisted surgery is not necessary for all peripheral nerve cases, we believe it can be applied to cases that involve nerves prone to anatomic variability or found in deeper locations. Utilization of this technique may advance the field through improved patient outcomes related to shorter operative times and smaller incisions. Cost savings associated with reduced operative time may also help promote use of the technique.

255. Development of a Sciatic Nerve Schwannoma Model for Surgical Simulation Using 3D Printing and Polymer Hydrogels

Jonathan Jay Stone, MD; Shivali Mukerji; G. Edward Vates, MD, $P h D$

Introduction: Surgical education relies heavily on training in an operating room but this unfortunately threatens patient safety and increases operative times. Peripheral nerve tumors are relatively rare leading to underprepared resident graduates. To fill training gaps, we used 3D printing technology and polymer hydrogels to develop a full-procedural model for resection of a sciatic nerve schwannoma.

Methods: All anatomical layers of a human posterior thigh were recreated with polyvinyl alcohol (PVA) hydrogels. First, virtual models of each component were generated using imaging from several patients with lower extremity nerve tumors. Injection molds were 3D printed for the tumor, tumor capsule, sciatic nerve, musculature, subcutaneous fat, and skin. PVA was selectively crosslinked to mimic the different mechanical properties of each element. The individual tissue layers were then anatomically assembled; creating a model that permitted skin-to-skin rehearsal. Conductive yarn was molded into the nerve in order to permit stimulation and detection of injury to nerve fibers.

Results: A realistic bleeding peripheral nerve tumor simulator was developed and fabricated that closely approximated the relevant anatomy and tactical feel of a live case. This complete model incorporated each step of the surgery, including delicate dissection of the anatomic planes, identification of proximal and distal parent nerve, subscapular dissection of the tumor, and multilayer closure. Reproducible measurements were made from the conductive yarn, which allowed the trainee to stimulate and find viable nerve fibers within the capsule. It also added in trainee assessment by detecting if there was stretch on the nerve or transection of nerve fascicles.

Conclusion: This model has great potential as an educational tool. The next step is to conduct a validation study to assess face, content and construct validity between experts and residents. We plan to use this model for boot-camp scenarios to supplement resident training of this rare procedure. 
256. Effectiveness of Distal Supercharge End-to-Side Nerve Repair for Neuroma In-continuity (NIC) Nerve Injury

Mustafa Nadi; Sudheesh Ramachandran, MCh; Yuval Shapira; Rajiv Midha, MD; Joanne Forden; Abir Islam; Guifang Guo

Introduction: Introduction Transfer of an intact motor nerve distal to the side of a proximally injured nerve has been termed Supercharge end to side transfer (SETS). The use of SETS close to the neuromuscular junction could potentially lead to earlier muscle re-innervation and better functional outcome in NIC injuries where primary repair is not required. Aim To investigate distal SETS repair for management of NIC injuries in rodents for functional and regeneration outcomes.

Methods: Male Lewis rats ( $n=35)$ were assigned to one of five groups for unilateral sciatic nerve surgeries: tibial NIC with distal peroneal-tibial SETS (treatment group), tibial NIC without SETS (injury control), intact tibial nerve with peroneal transection (positive control), tibial nerve transection with SETS (ETS control) and tibial nerve transection without SETS (negative control). Recovery was evaluated biweekly (upto week-12) using ladder rung skilled locomotion task and monthly by electrophysiological assessment utilizing compound muscle action potentials (CMAP). At study endpoint, the contribution of ETS repair was analyzed by retrograde labeling from the distal tibial nerve to assess tibial and peroneal motorneuron contribution.

Results: CMAP difference at week-12 between ETS groups and the negative control was statistically significant, suggestive of a robust motor neuronal regeneration across the NIC and/or SETS. Behavioural analysis did not show significant differences in the treatment group and ETS group. Retrograde labelling from the distal tibial nerve showed significant increase in the treatment and the ETS control groups suggestive of significant collateralisation from the peroneal nerve via SETS. Improvement in CMAP and retrograde labelling were found to be poorly reflective of functional outcome, possibly due to simultaneous activation of antagonistic muscles innervated by the tibial and peroneal nerves.

Conclusion: SETS is an effective strategy to enhance motor re-innervation in incomplete nerve injuries, but collateral innervation may be detrimental if an antagonistic motor donor is chosen.

\section{Trends in Lower Extremity Nerve Injuries Using the National Inpatient Sample}

Michael Jensen; Ryan Watkins, BS; Michael Karsy, MD, PhD; Jian Guan, MD; Mark Alexander Mahan, MD

Introduction: Medical care costs have risen substantially; however, the relationship of peripheral nerve injury to medical costs is not well known. We seek to understand the clinical and epidemiological factors associated with peripheral nerve injuries, and how surgical treatment may contribute to cost.

Methods: Using the National Inpatient Sample(NIS), we compared lower extremity peripheral nerve injuries, including lumbosacral plexus and single nerve injuries, between early (2001-2002) and late (2011-2012) patient groups. Statistical analysis was performed using Prism software.

Results: We randomly sampled a total of 162 early and 150 late group patients from the NIS showing a similar national incidence (106:1,000,000 vs. 97:1,000,000 patients). The average age of the patient was $41.3 \pm 20.4$ years, $64.7 \%$ were male, and the average length of stay was $7.4 \pm 10$ days. When comparing the two epochs, there was an increase in the number of peripheral nerve procedures billed in the late group when compared to the early group, though not statistically significant (11 early vs. 22 late, $\mathrm{p}=0.06$ ). There was a substantial difference in cost when comparing the two epochs $(\$ 36,284$ early vs. $\$ 81,369$ late, $\mathrm{p}<0.01$. There was no difference in associated lower extremity fracture ( 4.2 vs. $4 \%, \mathrm{p}=0.9)$, or muscle/tendon procedures (4.6 vs. $3.7 \%, p=0.4$ ). However, there was a significant increase in transfusions in later years $(1.4$ vs. $2.8 \%, \mathrm{p}=0.005)$, suggesting a greater proportion of other associated traumas in later patients.

Conclusion: The cost of medical care associated with lower extremity peripheral nerve injuries has significantly risen over a tenyear period. This is potentially due in part to the repair of associated injuries as well as nerve repair. The advance of surgical techniques, surgeon training and appreciation of outcomes from earlier surgical intervention may underlie the increased cost, with the aim of improving patient outcomes.

258. Trends in Upper Extremity Nerve Injury Using the National Inpatient Sample Database

Ryan Watkins, BS; Michael Jensen; Michael Karsy, MD, PhD; Jian Guan, MD; Mark Alexander Mahan, MD

Introduction: Peripheral nerve injury can be underappreciated in patients with polytraumatic injuries. We sought to understand trends in upper extremity nerve injuries, treatments and costs.

Methods: Patients with upper extremity (UE) nerve injury from 2001-2002 and 2011-2012 were compared using the National Inpatient Sample. Patients with either brachial plexus or upper extremity nerve injuries were included. Patient demographics, hospital characteristics, associated diagnoses, treatments, and disposition were evaluated and compared between periods.

Results: A random sample of 593 and 469 patients from the early and late years were characterized, respectively, representing a decrease in incidence $(387 / 1,000,000$ vs $306 / 1,000,000 \mathrm{p}<0.001)$. Demographically, the epochs were not statistically different in mean age of $37.9 \pm 18.6$ years or gender $(73.3 \%$ male). Most patients were admitted with trauma designation, with a significant decrease in the later epochs $(75.7 \%$ vs. $48.4 \%, p<0.0001) .30 .3 \%$ of patients had damage to a main artery in the UE and $39.5 \%$ of patients had an open wound with visible tendon involvement. Treatment of the nerve injury did not statistically differ between epochs for nerve transposition (1.9 vs. $2.6 \%, p=0.53$ ), nerve decompression ( 3.9 vs. $5.5 \%, p=0.24$ ), nerve suturing ( 40.3 vs. $35.4 \%$, p- 0.11 ), or other neuroplasty ( 7.9 vs. $6.4 \%, p=0.4)$. There was a significant increase in nerve grafts $(0.7$ vs. $5.3 \%, \mathrm{p}=0.0001)$ for later years. Discharge disposition to home or self-care was significantly lower in later years (81.9 vs. $50.1 \%$, $\mathrm{p}=0.0001)$. Charges were significantly increased in the later epoch $(\$ 26,082 \pm 44,969$ vs. $\$ 72,530 \pm 105,182 \mathrm{p}<0.05)$.

Conclusion: While the incidence of UE peripheral nerve injury seems to have decreased, the use of nerve graft procedures has increased - suggesting a trend towards higher level reconstructive approaches on a national scale. With majority of cases being referred to large teaching hospitals, peripheral nerve injury should be included within residents' curricula to help improve outcomes via recognition, referral, and primary treatment.

\section{An Overlooked Cause of Neck Pain: Calcific Tendinitis of the Longus Colli}

\section{Hyun-Seung Rhyu; Jung-Kil Lee, MD; Bong Ju Moon; Ki-Young Choi}

Introduction: Acute calcific tendinitis of the longus colli muscle is a rare clinical entity that causes severe neck pain. This entity is not well recognized due to its non-specific presentation such as acute neck pain, neck stiffness, odynophagia or dysphagia. The importance of this disease with a review of the literature is presented.

Methods: Acute calcific tendinitis of the longus colli muscle is an inflammatory condition caused by deposition of calcium hydroxyapatite in the superior oblique tendon fibers of the longus colli muscle. It can be misdiagnosed as other life-threatening conditions including retropharyngeal abscess, resulting in unnecessary medical or surgical interventions. 
Results: We retrospectively reviewed the clinical data, radiological features, and laboratory reports of eight patients who were diagnosed with acute calcific tendinitis of the longus colli muscle presented in this article were seen at our institution between April 2008 and March 2015. We describe the clinical presentation, diagnosis, and treatment of acute calcific tendinitis of the longus colli muscle.

Conclusion: There were 5 men and 3 women who ranged in age from 41 to 49 years (mean age: 44.5 years). The associated symptoms included neck pain, stiffness, odynophagia, and headache. The duration of symptoms varied from 2 days to 1 week. All patients showed calcific deposition inferior to the anterior arch of the atlas, and prevertebral effusion extending from $\mathrm{Cl}$ to $\mathrm{C} 4$. All patients were treated with NSAIDs and immobilization with a cervical brace, and they showed complete resolution of symptoms within 1 week.

\section{Ovarian Hormones Differentially Affect Pain Responsiveness in Plasma Membrane Calcium ATPase 2 (PMCA2) Heterozygous Mice}

Veronika Khariv, BS, MS; Li Ni; Robert F. Heary, MD; Stella Elkabes, $P h D$

Introduction: Our earlier studies indicated that PMCA2, a neuronal calcium extrusion pump, is a novel modulator of pain responses and dorsal horn (DH) pain processing in a sex-dependent manner. Female, but not male, PMCA2+/- mice (HT) are more sensitive to mechanical pain than female PMCA2+/+ mice (WT) and show unique molecular changes in the $\mathrm{DH}$. As ovarian hormones and especially 17beta-estradiol (E2) have been implicated in the modulation of pain, we determined whether ovarian hormone depletion and E2 replacement show differential effects on mechanical pain in WT versus HT mice.

Methods: Two-month-old female WT and HT littermates underwent ovariectomy (OVX) or SHAM surgery. Ovariectomized mice received silastic capsules containing vehicle (sesame oil) or E2 $(25 \mu \mathrm{g} / \mathrm{mL})$, immediately following surgery. Two weeks later, mechanical sensitivity was assessed as a measure of spinally mediated pain using the von Frey filament test. ANOVA was used for statistical analysis.

Results: Mechanical sensitivity was significantly increased in ovariectomized WT mice compared to SHAMs $(\mathrm{p}<0.05, \mathrm{n}=9-10)$. In contrast, mechanical sensitivity trended to decrease in ovariectomized HT mice compared to SHAMs ( $p=0.06, n=12-13$ ). E2 replacement restored mechanical sensitivity to sham values in ovariectomized WT mice but had no effects on ovariectomized HT mice.

Conclusion: Depletion of ovarian hormones affects mechanical pain responses in WT and HT mice in an opposite manner. The induction of hyperalgesia in WT mice following OVX together with its reversal by E2 replacement, implies that E2 exerts an analgesic effect in the intact WT mice. In contrast, in HT mice, the reduction in mechanical sensitivity following OVX implies that ovarian sex hormones have a hyperalgesic effect in the intact mice. The lack of effects following E2 replacement also suggest that E2 is not enough to modulate pain responsiveness in HT mice. Additional ovarian hormones such as progesterone may be necessary to affect pain responses in the HT mice.

262. A Novel Resorbable, Osteoconductive Tetracalcium Phosphate - Phosphoserine Bone Adhesive for Spinal Fusion: Initial In Vivo Studies in a Rabbit Posterolateral Fusion Model

Howard Eisenbrock, DO; Brian J. Hess, BS, MBA; George W. Kay, DMD, MMSc; Michael C Brown, BS; David J. Kosh; Andrey Marchenko, BS; Jonathan R. Slotkin, MD; Eric J. Woodard, MD

Introduction: We describe initial in vivo investigations of a novel calcium and phosphoserine-based bone adhesive. This material shows a unique constellation of properties including rapid self-setting, immediate tensile and load-bearing strength, and notable capacity to adhere to both bone and metal. This material is gradually biodegraded and replaced by bone through bone growth and remodeling. The aim of this study was to examine the tensile strength of this material and its prospective radiographic findings in a rabbit posterolateral fusion model.

Methods: Twelve adult New Zealand White rabbits underwent testing at the L5-6 level. The transverse process (TP) were exposed and decorticated at L5 and L6 bilaterally using a high speed drill. Rabbits received either freeform syringe-injectable or preformed solid-state material. One rabbit was used as a negative control. All rabbits were analyzed using Cone Beam Computed Tomography (CBCT) every three weeks. Selected animals were chosen for biomechanical testing at 3,6, and 10 weeks. Tensile strength testing was done at both L5/6 (experimental level) and L4/5 (control).

Results: In this study our $\mathrm{T}=0$ data shows a $1.66 \mathrm{x}$ increase in tensile strength with freeform syringe-injectable material compared to control (131.4 N vs. $79.1 \mathrm{~N})$. Later data points show an even greater increase in strength as compared to control: $283.7 \mathrm{~N}$ at 10 weeks with freeform and $257 \mathrm{~N}, 283.7 \mathrm{~N}$, and $288.5 \mathrm{~N}$ at 3,6, and 10 weeks respectively for preformed material.

Conclusion: This material has shown initial promise to be a valuable adjunct for posterior spinal fusion as evidenced by initial strength testing and imaging data. Ongoing in vivo and in vitro testing will determine what role it may have in future spine surgery.

\section{Bioresorbable Electrical Stimulator for Improved Bone Regeneration}

Jawad M. Khalifeh, BS; Zohny Zohny, MD; Manu Stephen; William R. Johnston, BS; Paul Gamble; Youchun Zeng, BS; Matthew R. MacEwan, BSE, PhD; Wilson Zachary Ray, MD

Introduction: Non-union/Pseudoarthrosis can be a significant problem following instrumented spinal fusion. Direct current electrical stimulation (DCES) of bone growth represents a unique surgical adjunct to promote and accelerate bone healing. Existing spinal fusion DCES systems utilize permanent electronic components, are invasive, and carry significant safety risks. The present study describes the design and implementation of a novel implantable bioresorbable DCES device and its application to a rodent femoral injury model.

Methods: Non-critical femoral defects were created in 15 Lewis rats. Animals were randomized into three treatment groups ( $\mathrm{n}=5$ each). Group I did not receive any stimulation, and Groups II and III received daily continuous 50uA DCES through permanent and bioresorbable femoral electrodes, respectively. All animals were euthanized two weeks post implantation and the injured femurs were harvested. Qualitative and quantitative analysis for regional bone formation was performed using morphometric and density parameters, as assessed by high resolution micro-computed tomography (micro-CT).

Results: Micro-CT morphometric analysis demonstrated increased overall bone formation, total tissue and bone volume, bone volume fraction, cross-sectional area, and cortical bone area after two weeks of DCES (Figure 1). The performance of bioresorbable stimulators compared favorably with that of permanent devices.

Conclusion: The results of the study suggest a trend toward increased bone formation with DCES in line with previous work, and highlight the possibility for integrating implantable bioresorbable technology to enhance bone healing. Further work is needed to examine the impact of varying DCES on osteogenesis and the optimization of bio-degradable hardware systems. 


\section{Design and Evaluation of Bioresorbable Spinal Cord Stimulator}

William R. Johnston, BS; Zohny Zohny, MD; Manu Stephen; Jawad M. Khalifeh, BS; Youchun Zeng, BS; Matthew R. MacEwan, BSE, PhD; Wilson Zachary Ray, MD

Introduction: Spinal cord injury (SCI) is a devastating condition that results in substantial impact on quality of life and longevity [1]. Recovery is rare and limited intervention exits to restore functional use of paralyzed limbs. Recent studies indicate chronic epidural stimulation may provide a degree of functional recovery following SCI [2-5]. However, these therapies rely on permanent electronic hardware that carry high infection risk [6-7]. Here we evaluate the longevity and functionality of a novel partially bioresorbable spinal cord stimulator to evoke motor evoked potentials (MEPs) and somatosensory evoked potentials (SSEPs) in a rat model, as a potential clinical solution to improve SCI therapies.

Methods: The functionality and longevity of the device was tested by stimulating the spinal cord via the device and recording MEPs and SSEPs at varying amplitudes each day. In addition, recordings were taken without stimulation being delivered to gather baseline signal as comparison. After the devices we no longer capable of delivering adequate stimulation, they were removed and the spinal cords explanted for histological analysis.

Results: Four devices have been successfully implanted, with two lasting seven days, one lasting eleven days, and another lasting twenty eight days. MEPs and SSEPs were successfully recorded for each at varying frequencies and amplitudes, with a significant difference demonstrated between the stimulation and no stimulation recordings. This demonstrates the capacity of the device to successfully initiate both motor and sensory responses over an extended time period. Figure 1 demonstrates the difference between the stimulation and no stimulation recordings for one device, in which MEPs were successfully recorded for 11 days and SSEPs for 9 days.

Conclusion: These results demonstrate the ability of our device to evoke MEPs and SSEPs for 1-3 weeks, indicating both longevity and functionality. This highlights the possibility of using resorbable technology in spinal cord therapies, providing an avenue to reduce infection and immune reaction risk. As device design is optimized, we expect greater and more consistent longevity and functionality to be achieved. Future studies will evaluate the ability of the device to assist in sensory and motor function recovery following SCI, over both acute and chronic time courses.

\section{Toll-like Receptor 9 Antagonism Inhibits Spinal Cord Astrocyte Proliferation and Migration}

Lun Li, MS, MD; Li Ni; Eliseo Eugenin, PhD; Robert F. Heary, MD; Stella Elkabes, $P h D$

Introduction: The glial scar has been considered as an impediment to axonal regeneration, albeit studies also show that astrocytes are necessary for axonal re-growth following spinal cord injury (SCI). The principal cell type that form the glial scar are proliferating and migrating reactive astrocytes, which secrete both helpful and harmful effectors. Modulation of astroglial proliferation and migration can alter the properties of the glial scar and thereby, influence the outcomes of SCI. Evidence indicates that Toll like receptors (TLRs), which are expressed by SC neurons, glia and infiltrating immune cells, play important roles in SCI. Our laboratory has previously shown that a TLR9 antagonist, CpG ODN 2088, administered intrathecally, improves the functional and histopathological outcomes of SCI, and attenuates the pro-inflammatory phenotype of SC astrocytes, in vitro, through direct actions. The current studies were undertaken to determine whether the TLR9 antagonist modulates astroglial functions pertinent to glial scar formation such as proliferation and migration.

Methods: Mixed glial cultures, derived from the SC of postnatal day 2-3 mouse pups were used to isolate astrocytes, which were passaged three times for $99 \%$ purification. Astrocyte cultures were incubated in Minimum Essential Medium containing 1\% heat inactivated fetal bovine serum, in the presence or absence of CpG ODN 2088. EdU, a thymidine analogue was used to label proliferating cells whereas a scratch-wound assay and live cell imaging were utilized to assess the astroglial migration.

Results: CpG ODN 2088 significantly reduced by $40 \%$ the number of proliferating astrocytes $(p<0.001 ; n=4)$. The antagonist also significantly decreased the astroglial migration into the gap formed by the scratch. These effects necessitated TLR9 since CpG ODN 2088 did not affect the proliferation or migration TLR9-/- astrocytes.

Conclusion: Astroglial TLR9 antagonism inhibits both proliferation and migration, in vitro. Thus, CpG ODN 2088 has the potential of targeting astrocyte functions pertinent to glial scar formation.

266. Deficient Toll-like Receptor 4 Signaling Alters Chondroitin Sulfate Proteoglycan Expression Following Spinal Cord Injury in Mice

Viren Sahai Vasudeva, MD; Jared Groff; Nandhu M. Sobhana; Mariano S. Viapiano, PhD

Introduction: Following spinal cord injury (SCI), activation of the innate immune system occurs, drawing activated CNS macrophages expressing TLR4 receptors to the injury site. Surprisingly, mice with deficient TLR4 signaling pathways have worse locomotor outcomes following thoracic SCI when compared to wild type controls. The mechanism of this action however is unknown. We designed an experiment to explore if deficient TLR4 signaling influences the expression of chondroitin sulfate proteoglycans (CSPGs), which are the major structural components of the CNS extracellular matrix. Differential expression of CSPGs may then influence cell migration, axon extension, synaptogenesis, and functional recovery.

Methods: $\mathrm{C} 3 \mathrm{H} / \mathrm{HeJ}$ (TLR4 deficient) and $\mathrm{C} 3 \mathrm{H} / \mathrm{HeOuJ}$ (wild type control) mice were anesthetized and a laminectomy was performed at T9. Mice were designated to either a 'sham' group or 'SCI' group. The sham mice did not undergo any spinal cord injury. The SCI mice received a moderate spinal contusion injury using an electromechanical spinal contusion injury device. Fourteen days post-injury, spinal cords were isolated and blocked into three $1 \mathrm{~cm}$ segments centered on the impact site (rostral, epicenter, or caudal). Protein was isolated from tissue homogenates and western blotting was performed to test for expression of lectican (neurocan and brevican) and non-lectican (phosphacan and NG2) CSPGs. GFAP expression was used as a marker of astrogliosis.

Results: When comparing $\mathrm{C} 3 \mathrm{H} / \mathrm{HeJ}$ and $\mathrm{C} 3 \mathrm{H} / \mathrm{HeOuJ}$ spinal cord tissue, these experiments demonstrated that there were changes expression of full-length neurocan, N-terminal brevican, and NG2 that occurred not only at the injury site, but also in rostral and caudal sections. Neither phosphacan nor GFAP showed any change in expression in any of the treatment groups.

Conclusion: Although TLR4 signaling is thought to be primarily involved in activation of the innate immune system; these results show that it is also involved in regulating the structure of the extracellular matrix following SCI. 
267. A Toll Like Receptor (TLR) 9 Antagonist Restores Astroglial Glutamate Transporter Expression in the Dorsal Horn (DH) Following Spinal Cord Injury (SCI): Implications for BelowLevel Neuropathic Pain

Alexandra Pallottie; Ayomi Ratnayake; Li Ni; Robert F. Heary, MD; Stella Elkabes, PhD

Introduction: Astroglial glutamate aspartate transporter (GLAST) and glutamate transporter 1 (GLT-1) play important roles in the clearance of extracellular glutamate and have been implicated in glutamate-mediated hyperexcitability of dorsal horn (DH) neurons, a mechanism underlying central sensitization and neuropathic pain. We have previously reported that intrathecal treatment of mice sustaining a mid-thoracic spinal cord injury (SCI), with a toll-like receptor (TLR) 9 antagonist, CpG ODN 2088, limits the development of below-level neuropathic pain. The current study examined the effects of CpG ODN 2088 on GLAST and GLT-1 levels in the lumbar DH, a region involved in the processing of below-level chronic pain.

Methods: A severe mid-thoracic contusion injury was induced in female mice. Intrathecal CpG ODN 2088 administration started at 1 day post-injury (dpi) and was repeated at $48 \mathrm{hr}$ intervals until $28 \mathrm{dpi}$. Thereafter, the injury epicenter and lumbar $\mathrm{DH}$ regions were collected. All samples were assessed for protein expression by western blot analysis. A one-way ANOVA was used for statistical analysis.

Results: A mid-thoracic SCI reduced GLAST and GLT-1 protein levels within the lumbar DH at 28dpi $(\mathrm{p}<0.001)$. Treatment of injured mice with CpG ODN 2088 prevented the reduction in GLAST and restored GLT-1 to uninjured values. Neuronal glutamate transporter and receptor subunit levels were not affected by the antagonist. In contrast to the lumbar DH, GLAST and GLT-1 levels in the epicenter were not altered following CpG ODN 2088 treatment.

Conclusion: These findings indicate that the TLR9 antagonist affects regions remote from the injury epicenter and selectively modulates astroglial glutamate transporters following SCI, potentially, through direct effects on astrocytes.

\section{Spinal Cord Injury Disrupts Resting-State Networks in the Human Brain}

Ammar H. Hawasli, MD, PhD; Jerrel Rutlin; Rory K.J. Murphy, MD; Eric C. Leuthardt, MD; Joshua S. Shimony, MD, PhD; Wilson Zachary Ray, $M D$

Introduction: Despite 253,000 spinal cord injury (SCI) patients in America, there are few diagnostics and therapeutics after SCI. Magnetic resonance imaging provides limited non-structural information and insight into functional connectivity. Resting-state functional brain MRI (RS-fMRI) quantifies network connectivity through resting-state networks (RSNs) and allows detection of functionally-relevant changes during disease. Given a robust network of spinal cord afferents to the brain, we hypothesized SCI produces meaningful changes in brain RSNs.

Methods: Serial clinical assessments and RS-fMRIs were performed on 9 SCI patients. Blood oxygen-dependent RS-fMRI sequences were acquired. After preprocessing, registration and quality assurance, seed-based correlation mapping was performed using RSNs: default-mode, dorsal-attention, salience, control and somatomotor. Correlation maps were generated by extracting timecourse from each seed. Internal and cross connectivity of RSNs were compared to 36 controls using false-discovery rate-corrected Wilcoxon rank-sum tests.

Results: SCI altered brain network connectivity. SCI reduced connectivity within salience $(\mathrm{p}=0.016)$, somatomotor $(\mathrm{p}=0.038)$ and default-mode ( $\mathrm{p}=0.047, \mathrm{~N}=9)$ networks. Internetwork connectivity increased between control and somatomotor $(\mathrm{p}=0.041)$ and decreased between control and default-mode $(\mathrm{p}=0.031)$. Complete and incomplete SCI had different effects on RSNs. Connectivity within default-mode was disrupted after complete $(\mathrm{p}=0.049)$ but not incomplete $(p=0.45)$ SCI. After SCI, networks changed over time: connectivity increased between control and somatomotor but decreased between control and default-mode.

Conclusion: SCI disrupted RSNs in the human brain. SCI impaired connectivity within salience regions that filter/process sensory/cognitive inputs and activate executive-function. SCI also mitigated connectivity within primary motor/sensory regions and within the network most active at rest (default-mode). SCI disrupted functional connectivity between executive-control and sensorimotor regions but enhanced connectivity between executive-control regions and areas active during rest. Connectivity aberration were dependent on severity of spinal cord injury and changed over time. We expect brain connectivity changes after SCI will serve as biomarkers to predict functional recovery following a spinal cord injury and guide future therapy.

\section{Increased Cortical Plasticity is a Driver of Recovery of Function in Spinal Cord Injury}

David Gimbel, MD; Cafferty William, PhD; Stephen Strittmatter, $M D, P h D$

Introduction: Axonal outgrowth and plasticity are limited by central nervous system myelin while peripheral nervous system myelin is a viable substrate for axonal growth after injury. This is a significant reason that stroke and spinal cord injury are particularly devastating. Nogo Receptor $(\mathrm{NgR})$ is the receptor for the inhibitory oligodendrocyte membrane proteins Nogo, OMGP, and MAG. Genetic deletion of $\mathrm{NgR}$ in mice has been shown promote functional recovery in spinal cord injury. However, in all rodent spinal cord injury models the degree of new axonal outgrowth below the site of injury is modest in comparison to the degree of functional recovery. These observations suggest that the driver for functional recovery must be upstream of the site of injury. We hypothesize that increased cortical plasticity, coopting uninjured fiber tracts, is the driver of return of function in spinal cord injury.

Methods: Cortical windows were created on YFP C57B6 mice genetically engineered with and without $\mathrm{NgR}$. These mice were then serially imaged before and after dorsal hemisection and through the recovery period. Motor cortex dendritic spine turnover was then quantified. Additionally, pilot experiments were performed with GCaMP mice with motor cortex windows measuring calcium channel activity before and after injury.

Results: Mice with spinal cord injury have increased spine turnover in motor cortex when $\mathrm{NgR}$ is genetically deleted. Additionally, preliminary studies show in the setting of pyridotomy there is a change in calcium signaling patterns with ambulation.

Conclusion: Given the degree of modest axonal outgrowth below the site of injury it is reasonable to conclude that the driver of functional recovery after SCI is upstream. Our data indicate that cortical plasticity on a structural and functional level is affected by spinal cord injury.

270. Do Somatosensory Evoked Potential (SSEP) Changes Increase the Probability of a Postoperative Neurological Deficit Following Posterior Cervical Decompression Surgery?

Gurpreet Surinder Gandhoke, MD; Jaspreet Kaur; Yash Kalpesh Pandya; Zachary J. Tempel, MD; Donald Crammond; Jeffrey Balzer, PhD; Parthasarthy Thirumala; Adam S. Kanter, MD

Introduction: Neuromonitoring changes that occur in an operation may or may not recover to baseline values; we sought to 
determine if a persistent SSEP change increased the likelihood of a new neurological deficit.

Methods: Retrospective analysis of an observational cohort of prospectively collected data on 4489 patients over a 3-year period was performed. Subset of 1372 patients undergoing posterior cervical decompression surgery with or without fusion was analyzed. All patients had SSEP monitoring during the procedure. SSEP changes were classified as transient or persistent. New neurological deficits in the immediate post-operative period $(24 \mathrm{hr}$.) were identified.

Results: Of 1372 patients, 786 (57\%) were male and 586 (43\%) female. Mean age 57.9 years (Std. Dev. 6.49, range 2-94), mean weight $84.7 \mathrm{Kg}$. (Std. Dev. 22.54, range 27.5-200), Mean BMI 29.15 (Std. Dev. 7.18, range 13.6-80.6), Mean length of stay 5.65 days (Std. Dev. 6.49, range 1-95). Ninety-six patients (7\%) had significant SSEP changes, of which $13(13.5 \%)$ developed a new post-operative neurological deficit. One thousand two hundred and seventy six (1276) did not have a significant SSEP change and 49 (3.8\%) of these patients developed a post-operative neurological deficit $(\mathrm{p}<0.0001)$. Of the 96 patients with SSEP changes, 65 were transient, eight of which had a new deficit; five of the 31 with persistent SSEP changes had a new deficit $(\mathrm{p}=0.751)$. There was no statistically significant difference in the development of a new neurological deficit in patients with normal (33/626) or abnormal (26/658) baseline SSEPs $(p=0.287)$.

Conclusion: Any change in SSEPs, transient or persistent, is a statistically significant predictor in the development of a new neurological deficit in patients undergoing posterior cervical decompression surgery. Multimodality monitoring including motor potentials may be considered to detect predominantly motor deficits in patients without SSEP changes while long term follow-up may better elucidate the clinical relevance of transient versus persistent changes.

271. Chondroitinase ABC Pretreatment Enhances Functional Repair of the Chronically Injured Cervical Spinal Cord by Induced Pluripotent Stem Cell Derived Neural Stems Cells

Christopher Saagar Ahuja, MD; Hidenorii Suzuki; Ryan Salewski; Lijun Li; Kajana Satkunendrarajah; Narihito Nagoshi, MD, PhD; Michael G. Fehlings, MD, PhD, FRCS(C), FACS

Introduction: Cervical spinal cord injury (cSCI) is associated with devastating physical, social, and vocational consequences for patients and families. Intraparechymal transplantation of induced pluripotent stem cell-derived neural precursor cells (iPS-NPCs) is an exciting therapeutic strategy, however, the vast majority of patients are in the chronic phase of their injuries where dense chondroitin sulfate proteoglycan (CSPG) scarring potently inhibits regenerative cell migration and neurite outgrowth. Chondroitinase ABC (ChABC) is a bacterial enzyme capable of rapidly degrading CSPGs and has been shown to enhance recovery after acute SCI in animal models.

Methods: We investigated a novel approach administering 7 days of intrathecal ChABC to 'unlock' the chronic injury microenvironment prior to intraparenchymal murine iPS-NPC transplant. We compared five groups: (1) ChABC, (2) NPCs, (3) ChABC + NPCs, (4) vehicle alone and (5) sham injury (laminectomy alone). Behavioural assessments were completed weekly for 9 weeks post-transplant.

Results: We found that initial ChABC pretreatment reduced CSPG scarring and dramatically improved iPSC-NSC graft survival $(7.8 \%$ vs $2.4 \%)$. This combined treatment group also resulted in significantly higher numbers of functionally-critical cholinergic neurons (ChAT+, NeuN+; 7516 \pm 832 ) being present compared to NPC transplant alone $(4904 \pm 1243)$ or ChABC alone $(5890 \pm 552)$. Functionally, only the ChABC + NPC group demonstrated sustained forelimb grip strength recovery versus controls $(p<0.05)$.

Conclusion: This study provides key evidence that ChABC pretreatment can 'unlock' the chronically injured spinal cord niche to dramatically enhance NPC-mediated recovery. Furthermore, this work opens new avenues for exploration towards an effective and translatable treatment for our patients.

\section{SMaRT Human Stem Cells to Optimize Regeneration of the Traumatically Injured Spinal Cord}

Mohamad Khazaie; Christopher Saagar Ahuja, MD; Michael G. Fehlings, $M D, P h D, F R C S(C)$, FACS

Introduction: Cell-based regeneration of the traumatically injured spinal cord is an exciting therapeutic strategy as engrafted cells can reform functional neural circuits, remyelinate denuded axons, and provide substantial local trophic support. Unfortunately, several barriers to regeneration exist including poor graft survival, low grafthost integration, and impeded neurite outgrowth within the potently inhibitory chondroitin sulfate proteoglycan (CSPG) rich glial scar.

Methods: We have genetically engineered human induced pluripotent stem cell-derived neural precursor cells (hiPS-NPCs) into three unique lines of Spinal Microenvironment Modifying Regenerative Therapeutic (SMaRT) cells to directly address these barriers.

Results: (1) We generated pro-survival glial-derived neurotrophic factor (GDNF) expressing SMaRT cells which demonstrate enhanced survival $(21.3 \%$ vs $12.2 \%)$ in a clinically-relevant clip-contusion model of cervical spinal cord injury (cSCI). (2) We also established a line of 'spinal cord identity' SMaRT cells which are transcriptomically and phenotypically distinct from typical 'brain identity' NPCs to allow greater integration into the host cord. (3) Finally, we used non-viral techniques to produce SMaRT cells capable of efficiently degrading CSPGs in their local environment through inducible expression of enzyme Chondroitinase ABC (ChABC).

Conclusion: Together, these data represent key milestones towards an optimized human stem cell therapy for cSCI. By clearly establishing the therapeutic effects of each of these individual SMaRT cell lines, we can move towards a synergistic combinatorial strategy which exploits the capabilities of all three lines to overcome the multifaceted challenges to regeneration in cSCI.

\section{Neuromodulation for Chronic Cervical Spinal Cord Injury: Electromyogram Features of Promoted Upper-limb Volitional Function}

Nicholas Au Yong, MD, PhD; V. Reggie Edgeton; Morteza Modaber, MD; Erika Morikawa; Sharon Zdunowski; Melanie Sarino; Majid Sarrafzadeh, PhD; Marc Nuwer; Roland R. Roy; Yury Gerasimenko; Daniel C. Lu, MD

Introduction: Neuromodulation through electrical stimulation has been clinically employed in the central nervous system for the treatment of movement disorders and pain. More recently, epidural stimulation (ES) for the restoration of lower-limb motor function has been shown in spinal cord injured human subjects. In this study, we demonstrate that ES can also be applied to human subjects with chronic cervical cord injuries for promoting volitional hand function. Electromyogram features of hand function recovery was characterized to elucidate the underlying mechanism.

Methods: Two chronic ( $>18$ mo from injury) American Spinal Injury Association (ASIA) Impairment Scale (AIS) B subjects, implanted with cervical epidural array of 16 electrodes for chronic pain, were evaluated for improvement of handgrip control with ES. A handgrip force measurement device, in conjunction with a computer program, was used to measure voluntary hand control and grip characteristics. Surface electromyogram activity was measured concurrently during testing. Clinical metrics were also assessed throughout the study. 
Results: Hand control and force production improved in both subjects, as compared to baseline function, and were sustained even with cessation of stimulation. Improved hand function was associated with increases in electromyogram amplitudes. ES produced evoked responses predominantly during voluntary effort. Amount of force produced corresponded best with combined stimulus evoked (i.e. direct stimulation) and non-evoked electromyogram activity, as opposed to stimulus evoked alone.

Conclusion: Cervical electrical modulation merits further investigation as a means to improve hand volitional function in patients with chronic tetraplegia. These results suggests that the cervical motor circuitry, while operationally dissimilar to the conceptualization of the locomotor central pattern generator, is also amenable to neuromodulation through electrical stimulation. Features of electromyogram activity point to a mechanism whereby electrical stimulation augments the excitability of premotor circuits by shifting the network's state closer to motor threshold.

\section{Rehabilitation of Hand Function After Spinal Cord Injury Using a Novel Handgrip Device: A Prospective Cohort Study}

Haydn Hoffman, MD; Tiffany Sierro; Tianyi Niu, MD; Melanie Sarino; Majid Sarrafzadeh, PhD; David L. McArthur; Reggie Edgerton, PhD; Daniel C. Lu, MD

Introduction: Activity-based therapy (ABT) for patients with $\mathrm{SCI}$, which consists of repetitive use of muscles above and below the spinal lesion, improves locomotion and arm strength. Less data has been published regarding its effects on hand function. We sought to evaluate the effects of a weekly hand-focused therapy program on hand strength and function in a SCI cohort.

Methods: Patients with SCI were enrolled in a weekly program that involved activities with the MediSens (Los Angeles, CA) handgrip. These included maximum voluntary contraction (MVC) and a tracking task that required each subject to adjust his/her grip strength according to a pattern displayed on a computer screen. For the latter, performance was measured as mean absolute accuracy (MAA). The Spinal Cord Independence Measure (SCIM) was used to measure each subject's independence prior to and after therapy.

Results: Seventeen patients completed the program with average participation duration of 21.3 weeks. The cohort included patients with ASIA A $(n=12)$, AIS B $(n=1)$, AIS C $(n=2)$, and AIS D $(n=2)$ injuries. The MVC scores for motor complete subjects increased from $2.6 \mathrm{~N}$ to $3.7 \mathrm{~N}$ and in motor incomplete subjects, the scores increased from $4.1 \mathrm{~N}$ to $21.2 \mathrm{~N}$, but neither improvement was significant. Both groups showed significant improvement in MAA by the end of the study (motor complete: $5.7 \%$ to $20.7 \%, \mathrm{p}=.001$; motor incomplete: $12.8 \%$ to $35.1 \%, \mathrm{p}=.04$ ). At the end of the study, the average SCIM score for the motor complete group was unchanged. In the motor incomplete group it increased 2.75 points, but this was not significant.

Conclusion: A weekly handgrip-based ABT program is feasible and efficacious at increasing hand task performance in subjects with SCI. Those with incomplete motor injuries demonstrate larger taskspecific improvements than subjects with complete motor injuries.

\section{The Effects of Hypothermia on Functional Outcomes} Following Simulated Iatrogenic Spinal Cord Injury

Ahmed Jorge; Parthasarathy D. Thirumala; Shreya Umredkar; Erika Fish; Rohan Krishnan

Introduction: Spinal cord injuries accounts for the majority of neurosurgical procedures in the United States. Furthermore, data demonstrate that iatrogenic spinal cord injuries (iSCI) are potential complications. The incidence rate of iSCI varies from $0.57 \%$ to
$5.93 \%$ depending on among other factors age (adult vs pediatric) and initial injury etiology (1) (2). Examples of these perioperative injuries include direct traumatic injury from instrumentation, unintentional compressive injury during deformity correction or indirectly injury of surrounding vasculature. Outcomes vary from radicular pain to paraplegia or quadriplegia; moreover, in adolescent patients, impaired learning abilities (3) and depression have been documented (4).

Methods: Female Sprague Dawley rats were randomly divided into two groups: a hypothermia group $\left(32^{\circ} \mathrm{C}\right)$ and a normothermia group $\left(36^{\circ} \mathrm{C}\right)$. After anesthetizing the rats, a laminectomy was performed at T8. The group assigned to the iSCI was further contused with an Infinite Horizon impactor at $200 \mathrm{kdyn}$. After the contusion was performed, both groups received an iSCI. After 2 hours under hypothermia, the rats were rewarmed. The sham surgery group experienced the same procedures except the contusion. Locomotor scores were collected one day after the surgery and weekly for six weeks post surgery.

Results: After 35 days, the mean BBB score for the rats that underwent hypothermia was 11.6 (left hindleg). In contrast, the mean BBB score for the rats that were kept under normothermia was 4.3. A two sided t-test comparing the two groups yields a statistically significant difference between the two means of 7.2 points with a $\mathrm{p}$-val $=.02$.

Conclusion: Modest hypothermia before an iSCI does improve the neurophysiological functional outcomes as tested by the BBB locomotor test in Female Sprague Dawley rats.

276. Interim Safety and Efficacy Findings From the SCiStar Study. A Phase 1/2a Trial of Human Embryonic Stem CellDerived Oligodendrocyte Progenitor Cells (AST-OPC1) in Patients with Subacute Cervical Spinal Cord Injury

Richard G. Fessler, MD, PhD; Donald Leslie, MD; Gary K. Steinberg, MD, PhD; Charles Y. Liu, MD, PhD; Shekar N. Kurpad, MD, PhD; Kevah Khajavi, MD; Jane Lebkowski, PhD; Edward Wirth, $M D, P h D$

Introduction: The initial clinical safety of AST-OPC1 was evaluated in a phase 1 trial that enrolled five patients with neurologically complete T3-T11 thoracic spinal cord injuries (SCI). Based on the favorable safety data from that study, a phase 1/2a trial (SCiStar Study) was initiated to evaluate the safety and activity of AST-OPC1 in patients with motor complete ASIA Impairment Scale A or B (AIS-A or B) C5-C7 cervical SCI.

Methods: The SCiStar Study is evaluating three escalating doses of AST-OPC1 $(2,10, \& 20$ million cells) administered via direct intraparenchymal injection between 14 and 30 days post-SCI. Enrollment of Cohort 1 ( $\mathrm{N}=3$ AIS-A patients, 2 million cell dose) and Cohort 2 ( $\mathrm{N}=6$ AIS-A patients, 10 million cell dose) has been completed and enrollment in the remaining study cohorts is in progress. Subjects are being followed for 1 year under the main study protocol and will be followed for an additional 14 years under a long-term follow up protocol.

Results: To date, there have been no intraoperative complications or serious adverse events (SAEs) related to AST-OPC1, the injection procedure, or immunosuppression with low-dose tacrolimus. Interim ISNCSCI exam data through Day 90 post-injection are currently available for all subjects in Cohort 1 and the first four subjects in Cohort 2. The mean UEMS improvement at Day 90 relative to baseline was 5.0 points in Cohort 1 and 9.5 points in Cohort 2. All subjects have improved at least one motor level, and 2 of the 4 subjects in Cohort 2 have improved two motor levels on at least one side.

Conclusion: The data to date suggest that AST-OPC1 can be safely administered to patients in the subacute period after severe cervical SCI, and a dose response effect on upper extremity motor recovery appears to be emerging by Day 90 post-injection. 
277. INSPIRE Study Update on 10 Acute Complete Spinal Cord Injury Patients Implanted with a Bioresorbable Polymer Scaffold

Domagoj Coric, MD; Nicholas Theodore, MD; Kee Duk Kim, MD; Patrick Hsieth; Wilson Zachary Ray, MD; K. Stuart Lee, MD, FACS; Travis Michael Dumont, MD; Kristen Neff; Lisa Crockett; Alex Aimetti, PhD; Thomas Ulich; Lorianne Masouka

Introduction: The INSPIRE study is an ongoing clinical trial of patients with complete paralysis following acute traumatic SCI (AIS A) implanted at the site of cord injury with a Neuro-Spinal Scaffold (NSS).

Methods: Patients are enrolled into the study within 96 hours after sustaining a complete thoracic T2-L1 traumatic paralysis. The NSS is implanted into the cord following durotomy and in some cases myelotomy if no open laceration is present. Patients are followed with serial MRI scans, neurologic evaluations and validated quality of life measures including bowel and bladder function for 6 months with long-term in-clinic follow up for up to 24 months.

Results: Ten patients have been implanted with a NSS. Eight patients are between 3 months and 2 year follow up. Two subjects died postoperatively one from underlying injuries and one from a stroke, neither of which were related to the NSS or implantation procedure. One subject converted from AIS A to AIS C, having recovered 18 points of motor function over the first year of recovery. Four additional subjects converted from AIS A to AIS B, with one subject continuing to recover sensory function over the first year of recovery. All subjects have substantially improved bladder function. Chronic cyst formation by MRI is low, with evidence of architectural preservation of tissue post-operatively.

Conclusion: The NSS is the first device implanted into the spinal cord in a series of acutely paralyzed patients. Thus far, the neurologic recovery of the patients has exceeded natural history expectations and there have been no adverse events associated with the Scaffold.

278. Efficacy and Safety of Riluzole in Acute Spinal Cord Injury (SCI): Rationale and Design of AOSpine Phase III Multi-center Double Blinded Randomized Controlled Trial (RISCIS)

Michael G. Fehlings, MD, PhD, FRCS(C), FACS; Branko Kopjar, MD; Robert G. Grossman, MD

Introduction: There is convincing evidence from the preclinical realm that the pharmacologic agent riluzole attenuates certain aspects of the secondary injury cascade leading to diminished neurological tissue destruction in animal SCI models. The safety and pharmacokinetic profile of riluzole have been studied in a multicenter pilot study in 36 patients. Efficacy of riluzole in acute human SCI has not been established.

Methods: This ongoing multi-center, international double-blinded phase III RCT will enroll 351 patients with acute C4 C8 SCI and ASIA Impairment Grade A, B or C randomized 1:1 to riluzole and placebo. Primary outcome is the change in ASIA Motor Score (AMS) between baseline and 180 days. Other outcomes include ASIA Upper and Lower Extremity MS; ASIA Sensory Score; ASIA grade; SCIM); SF-36v2; EQ-5D and GRASSP. Two-stage sequential adaptive trial statistical design has $90 \%$ power to detect 9 points difference in the ASIA Motor Score at one-sided alpha $=.025$.

Results: A matched cohort analysis performed in the Phase I study showed that riluzole treated cervical SCI patients experienced an additional 15.5 points in AMS recovery at 90 days post injury. Although the phase I study was underpowered to investigate efficacy the current phase III study is poised to definitive address this question. Subject enrollment for this trial began on October 1, 2013. To date, 57 subjects have been enrolled. Average age of the enrolled subjects is 49.7 (SD 16.3); 78\% males. ASIA at arrival and Pre-Injury status, ASIA Grade A (50\%), B (26\%), C (24\%). GRASSP 67.1 (SD
62.0), SF35v2 PCS 53.1 (SD 9.2) SF36v2 MCS 54.8 (SD 11.2).

Conclusion: This is a Phase III study of riluzole in acute SCI.

\begin{abstract}
279. Magnetic Resonance Imaging (MRI) does not Distinguish Between Contusion and Compound Cord Lesions Following Severe (AIS A) Traumatic Acute Thoracic Cord Injury: Intraoperative Microsurgical Observations from the INSPIRE Trial
\end{abstract}

Richard T. Layer, PhD; Alex Aimetti, PhD; Paul M. Arnold, MD; Maureen Barry, MD; Domagoj Coric, MD; Michael G. Fehlings, MD, PhD, FRCS(C), FACS; James D. Guest, MD, PhD; Robert F. Heary, MD; Patrick C. Hsieh, MD, MSc; Kee Kim, MD; K. Stuart Lee, MD, FACS; Lorianne K. Masuoka, MD; Kristin M. Neff, BS, MS; Zack Ray; Nicholas Theodore, MD; Thomas Ulich

Introduction: The Neuro-Spinal Scaffold, a biodegradable investigational device, is implanted via durotomy and often myelotomy into acutely injured spinal cord parenchyma to facilitate neural repair, plasticity, and possibly regeneration. The procedure enabled retrospective comparison of preoperative MRIs with visible post-durotomy/myelotomy cord pathology.

Methods: The INSPIRE trial enrolled patients with nonpenetrating severe (AIS A) spinal cord injury (T2-T12/L1 excluding complete cord transections). MRIs were obtained prior to open spine surgery. Following spine stabilization, durotomy enabled dorsal cord inspection. In subjects with intact cord surface, myelotomy enabled scaffold implantation.

Results: Scaffolds were implanted in 7 males and 2 females (aged $18-55$; 9-83 hours post-injury). Injuries were classified as 'contusiontype' (5 patients) with central necrosis, spared peripheral white matter, and intact pial surface, or 'compound-type' (4 patients) with elements of contusion, pial-disruption, laceration, maceration, and damaged peripheral white matter but preserved cord continuity. Following dorsal myelotomy of contusion-type injuries, gentle irrigation of loose necrotic/hemorrhagic debris revealed an intra-medullary cavity. Scaffolds implanted into the intra-medullary cavity of contusiontype injuries were generally not visible but could be identified using ultrasound. Scaffolds implanted into compound-type injuries remained visible. Pre-operative MRI did not distinguish between injury types. Of patients with contusion-type injury, one converted to AIS C and one to AIS B by 1 month. Of patients with compound-type injury, two converted to AIS B by 2 and 6 months. Two subjects died from causes deemed unrelated to scaffold implantation.

Conclusion: The Scaffold implantation procedure enabled comparison of pre-operative MRI with direct visual inspection of the acutely injured cord. Direct visual inspection, but not pre-operative MRI, enabled characterization of injuries as either 'contusion-type' or 'compound-type'. Both injuries were successfully implanted with the scaffold. AIS conversion was seen in both pathological types. Refinement of these observations will continue with future patient enrollment and could provide the basis of a novel clinical-pathological classification system.

\section{Hospital-Acquired Conditions in Spine Procedures}

Layton Lamsam, BS; Ian David Connolly; Anand Veeravagu, MD; John K. Ratliff, MD, FACS

Introduction: Beginning in October 2008, the Centers for Medicaid and Medicare Services (CMS) no longer reimbursed for 14 groups of inpatient complications known as Hospital-Acquired Conditions (HAC's)1,2. The spatial and temporal incidence of these complications in spine surgery remains largely unknown. Likewise, the effects of the CMS non-reimbursement policy on HAC's have not 
been examined in spine procedures.

Methods: The 2007-2014 MarketScan administrative database was used to query inpatient and outpatient records for patients receiving anterior cervical discectomy and fusion, lumbar laminectomy, lumbar discectomy, or posterior lumbar fusion, exclusively. Data on the incidence of HAC's, their geographic distribution, temporal distribution, and payment data were extracted. States with less than 100 observations (Hawaii, Vermont) and counties with 10 or fewer observations were excluded from individual analysis. HAC or nonHAC groups with less than 10 observations over a year-long time period were excluded from time series analysis.

Results: Geographically, there was a large variation in the HAC incidence from $3.5 \%$ (Wyoming) to $17.2 \%$ (West Virginia). Overall, HAC incidence increased from $1.7 \%$ in 2007 to $2.6 \%$ in 2014 . Falls $(67.0 \%)$, DVT/PE's $(19.5 \%)$, and surgical site infections $(9.6 \%)$ were the most common HAC groups. In 2007, median payments for Medicare patients with and without HAC occurrence were \$17,838 and $\$ 10,712$, respectively. Similarly, in 2014 they were \$42,474 and \$29,084. Median payments for admissions with HAC's were consistently higher than admissions without HAC's regardless of Medicare or private insurance.

Conclusion: The geographic incidence of HAC's is highly variable and warrants further research to correlate case volume and other systems-level factors. HAC incidence has not decreased over time after implementation of CMS's HAC non-reimbursement policy in 2008, and payments for admissions with HACs have remained consistently higher than their non-HAC counterparts.

\section{Risk Adjustment Modeling for Surgical Site Infection in the Michigan Spine Surgery Improvement Collaborative (MSSIC): Results from 7,150 Lumbar Patients}

Victor Chang, MD; Michael Bazydlo, MS; Lonni Schultz, PhD; David Nerenz, PhD; Jason M. Schwalb, MD, FACS; Muwaffak Abdulhak, MD; Stephen Bartol, MD, MBA, FACS

Introduction: MSSIC is a prospective registry, and a platform for quality improvement $(\mathrm{QI})$ of elective spine surgery across the State of Michigan. As one of its QI targets, the rate and risks for surgical site infection (SSI) were assessed. A risk adjustment model was developed to account for differences in patient risks across hospitals for further comparison.

Methods: Using the MSSIC registry, analyses of the rate of SSI, for lumbar surgery were performed. Potential risk factors for readmission were identified through literature review, bivariate analysis, and consultation with MSSIC surgeons. Odds ratios for each risk factor were estimated with multivariable logistic regression, and were used in generating the expected rate for each hospital. An adjusted rate for any given hospital was calculated by: (Observed rate/ Expected rate) * Overall MSSIC rate.

Results: A total of 7,150 patients across 21 hospitals were included in this analysis. The overall rate of SSI was $2.3 \%(n=179)$. Observed rates among hospitals ranged from $0.7 \%$ to $6.1 \%$ (Fig 1). The following risk factors were found to be statistically significant with increased risk: BMI; ASA $>2$; and length of surgery (Fig 2). Adjusted rates ranged from $0.6 \%$ to $7.4 \%$ (Fig 3). A total of $56 \%$ of patients who had an SSI were readmitted, and SSI accounted for $14 \%$ of all the 90-day hospital readmissions within MSSIC. There was a nearly 14-fold increase in the odds of readmission $(13.98, p<0.001)$ with an SSI vs no SSI.

Conclusion: While the overall rate of SSI is fairly low, any opportunity to lower the rate of SSI would represent a significant benefit. Within MSSIC there is a wide variability of SSI rates between hospitals, with potential for QI initiatives to lower SSI rates across the State of Michigan.
282. Lumbar Spine Surgical Site Infection Prevention with a Systematized Peri-operative Protocol: A Propensity Matched Multidepartmental Multiyear Retrospective Cohort Study

Vijay Yanamadala, MD; Thomas D. Cha, MD; Louis G. Jenis, MD; Joseph Schwab, MD; Bryan D. Choi, MD; Brian V. Nahed, MD; Jean-Valery Coumans, MD; Lawrence F. Borges, MD; John H. Shin, $M D$

Introduction: Surgical site infection (SSI) occurs after approximately $4 \%$ of degenerative lumbar spine cases and higher for complex spine surgery, including surgery for adult spinal deformity. It remains imperative to develop methods to reduce the incidence of SSI

Methods: After institutional review board approval, we retrospectively examined 1,089 consecutive lumbar spine surgeries performed between August 1, 2014 and August 1, 2016. Surgeons elected whether or not to use a peri-operative infection control protocol which included: (1) three days of chlorhexidine rinse prior to surgery; (2) chlorhexidine pre-prep scrub of the surgical site; (3) chlorhexidine prep with a 5-minute mandatory wait time; (4) intrawound vancomycin powder; and (5) iodine-impregnated antimicrobial film bandage. Nearest-neighbor propensity matching was performed according to demographic (age, sex, body mass index, co-morbidities, ASA grade, active smoking status) and surgical (operative time, estimated blood loss, number of levels fused) parameters. Statistical significance was $p<.05$ with a two-tailed t-test.

Results: 592 patients underwent surgery with this protocol, while 443 patients underwent only chlorhexidine prep. 446 patients (257 with protocol; 189 without) underwent lumbar decompressions. 589 patients (448 with protocol; 141 without) underwent lumbar fusion, with 54 patients (36 with protocol; 18 without) undergoing lumbar fusion involving six or more levels. Overall SSI rate was $1.1 \%(0.3 \%$, decompression; $1.6 \%$, fusion; $3.2 \%$, fusion $=$ six levels). SSI rate with the protocol was $0.9 \%(0.3 \%$, decompression; $1.1 \%$, fusion; $2.1 \%$, fusion $=$ six levels) compared to $1.3 \%$ without the protocol $(0.3 \%$, decompression; $1.9 \%$, fusion; $4.1 \%$, fusion $=$ six levels $)$. Multivariate regression analysis after propensity matching revealed a statistically significant difference in SSI rates for patients undergoing fusion involving six or more levels $(\mathrm{p}<.05)$, with a trend towards significance for all lumbar fusions.

Conclusion: A rigorous SSI prevention protocol can reduce infection rates, particularly in complex cases. Multi-institutional assessment will be essential to further corroborate its efficacy.

283. Posterior Cervical Spine Surgical Site Infection Prevention with a Systematized Peri-operative Protocol: A Propensity Matched Multidepartmental Multiyear Retrospective Cohort Study

Vijay Yanamadala, MD; Thomas D. Cha, MD; Louis G. Jenis, MD; Bryan D. Choi, MD; Jean-Valery Coumans, MD; Lawrence F. Borges, MD; John H. Shin, MD

Introduction: Surgical site infection (SSI) occurs after approximately $3 \%$ of cases in posterior cervical fusion cases. It remains imperative to develop methods to reduce the incidence of SSI.

Methods: After institutional review board approval, we retrospectively examined 189 consecutive cervical posterior spinal fusions performed between August 1, 2014 and August 1, 2016. Surgeons elected whether or not to use a peri-operative infection control protocol which included: (1) three days of chlorhexidine rinse prior to surgery; (2) chlorhexidine pre-prep scrub of the surgical site; (3) chlorhexidine prep with a 5-minute mandatory wait time; (4) intrawound vancomycin powder; and (5) iodine-impregnated antimicrobial film bandage. Nearest-neighbor propensity matching was performed according to demographic (age, sex, body mass index, 
co-morbidities, ASA grade, and active smoking status) and surgical (operative time, estimated blood loss, and number of levels fused) parameters. Statistical significance was $\mathrm{p}<.05$ with a two-tailed t-test.

Results: A total of 107 patients underwent posterior cervical fusion with this protocol, and 82 patients underwent only chlorhexidine prep. Overall SSI rate was $1.7 \%$ for this population. The overall SSI rate for patients undergoing the protocol was $0.9 \%$ compared to $2.4 \%$ for patients not undergoing the protocol $(\mathrm{p}=0.04)$. Our multivariate regression analysis after propensity matching demonstrated a $p$-value of 0.13 , suggesting a trend towards lower infections in patients undergoing the infection protocol.

Conclusion: We demonstrate that a rigorous protocol for SSI prevention can reduce infection rates. Multi-institutional assessment of our protocol will be essential to further corroborating the efficacy of this protocol in preventing posterior cervical surgical site infections after fusion procedures.

284. Prophylactic Use of Intra-Operative Vancomycin Powder and Post-Operative Infection: An Analysis of Microbiological Patterns in 1200 Consecutive Surgical Cases

Owoicho Adogwa, MD, MPH; Aladine A. Elsamadicy, BE; Amanda Sergesketter; Ankit Mehta, MD; Raul A. Vasquez, MD; Joseph S. Cheng, MD, MS; Carlos Antonio Bagley, MD; Isaac O. Karikari, MD

Introduction: Wound infections following spinal surgery for deformity place a high toll on patients, providers and the healthcare system. The prophylactic application of intra-operative vancomycin powder has been shown to lower the infection risk after thoracolumbar decompression and fusion for deformity correction. Few studies have assessed the microbiologic patterns of post-operative surgical site infections after prophylactic use of vancomycin powder. To investigate the incidence, epidemiology and pathogens associated with surgical site infections(SSI) after prophylactic use of intraoperative vancomycin powder.

Methods: All adult patients who underwent adult deformity reconstruction between 2011 and 2013 with a minimum of 3-months of clinical follow-up were retrospectively reviewed. All patients enrolled in the study received prophylactic crystalline vancomycin powder to the surgical bed. Baseline characteristics, operative details, rates of wound infection as well microbiologic data for each case were gathered by direct medical record review.

Results: Of 1200 consecutive operative spine cases performed for deformity between 2011 and 2013, 34(2.83\%) cases of SSI were identified. The mean \pm SD age was $62.08 \pm 14.76$ years. $29.41 \%$ had a history of diabetes and the mean BMI was $30.86 \pm 7.15 \mathrm{~kg} / \mathrm{m} 2$. The average dose of vancomycin powder was $1.41 \pm 2.77 \mathrm{~g}$ (range, $1-7 \mathrm{~g}$ ). Subfascial drains were placed in all patients. All SSI's occurred within 30-days of surgery, with deep wound infections accounting for $50 \%$ of all SSI's. Culture positive SSI's occurred in $74 \%$ of cases with the most common organisms being gram-negative infections such as Citrobacter Freundii, Proteus Mirabilis, Morganella Morgani and Pseudomonas aeruginosa. Only a minority of infections were due to gram-positive organisms. There were no adverse clinical outcomes related to the local application of vancomycin.

Conclusion: Our study suggests that in the setting of prophylactic vancomycin powder use, the preponderance of SSI's are caused by gram-negative or polymicrobial organisms. Further randomized control trials of prophylactic adjunctive measures is warranted to help guide choice of empiric-antibiotics.
285. Implementation of an Infection Prevention Bundle and Increased Physician Awareness Improve Surgical Outcomes and Reduce Costs Associated with Spine Surgery: A 10-Year Experience

Nitin Agarwal, MD; Prateek Agarwal, AB; Ashley Querry; Zachary J. Tempel, MD; Robert Max Friedlander, MD; Peter C. Gerszten, MD, MPH, FACS; D. Kojo Hamilton, MD; David O. Okonkwo, MD, $P h D ;$ Adam S. Kanter, MD

Introduction: Previous studies have demonstrated the efficacy of infection prevention protocols in reducing infection rates. However, there exists a lack of literature on the implementation of infection prevention bundles to reduce SSIs specifically in patients undergoing spine surgery. Furthermore, besides formal infection prevention bundles, the effect of physician awareness interventions on healthcare risks has not been adequately addressed. As such, we investigated the effects of an evolving infection prevention protocol augmented by increased physician awareness on spine surgery infection rates and resultant cost containment.

Methods: Neurological spine surgeons at a single academic institution were informed of spine surgery infection control measures as well as individual, independently adjudicated, spine surgery infection rates and rankings amongst their institutional peers. The groups were divided into those that actively employed recommended infection control measure protocols, and those that did not.

Results: With the implementation of postoperative surgical dressing measures and physician awareness, the postoperative spine surgery infection rate decreased by $45 \%$ from $3.8 \%$ to $2.1 \%$ for collaborative neurosurgeons (Risk Ratio $=0.55 ; 95 \% \mathrm{CI}, 0.32$ $0.93 ; p=0.03)$, resulting in an estimated annual cost savings of $\$ 291,000$. This reduction in infection rate was not observed for non-collaborative neurosurgeons, though the overall infection rate amongst all neurosurgeons decreased from $3.3 \%$ to $1.5 \%$ (Risk Ratio $=0.46 ; 95 \%$ CI, $0.280 .73 ; p=0.0013$ ). Thus, it appears as if the infection rate reduction amongst collaborative neurosurgeons was driving the reduction in the overall infection rate amongst all neurosurgeons.

Conclusion: A novel paradigm for spine surgery infection control combined with physician awareness methods resulted in significantly decreased infection rates and associated cost reduction. Thus, information sharing and physician engagement as a supplement to formal infection control measures results in improvements in surgical safety and costs.

286. Coagulation Profile as a Risk Factor for 30- Day Morbidity and Mortality Following Posterior Lumbar Fusion

Rachel S. Bronheim, BA; Eric Karl Oermann, MD; Samuel K. Cho, MD; John M. Caridi, MD

Introduction: Spinal fusion is one of the most common surgical procedures that routinely require transfusion, and posterior lumbar fusion (PLF) is traditionally associated with significant blood loss. There is research that suggests that abnormal coagulation profile is associated with postoperative complications, notably the need for blood transfusion. However, there is little in the literature that directly addresses the influence of coagulation profile on postoperative complications following PLF.

Methods: The American College of Surgeons National Surgical Quality Improvement Program database (ACS-NSQIP) was utilized to identify patients undergoing single level posterolateral lumbar fusion between 2006 and 2013. Multivariate analysis was utilized to identify associations between abnormal coagulation profile and postoperative complications.

Results: Low platelet count was an independent risk factor for organ space surgical site infections $(\mathrm{SSI})(\mathrm{OR}=6.0, \mathrm{P}<0.001)$, 
ventilation $>48$ hours $(\mathrm{OR}=4.5, \mathrm{P}=0.002)$, Acute renal failure $(\mathrm{OR}=5.8, \mathrm{P}=0.007)$, transfusion $(\mathrm{OR}=1.6, \mathrm{P}<0.001)$, sepsis $(\mathrm{OR}=$ $2.2, \mathrm{P}=0.037)$, reoperation $(\mathrm{OR}=2.5, \mathrm{P}=0.001)$, and death $(\mathrm{OR}=3.7$, $\mathrm{P}=0.049)$. High PTT was an independent risk factor for ventilation $>$ 48 hours $(\mathrm{OR}=5.6, \mathrm{P}=0.002), \mathrm{CVA} /$ stroke with neurological deficit $(\mathrm{OR}=5.1, \mathrm{P}=0.011)$, cardiac arrest $(\mathrm{OR}=5.4, \mathrm{P}=0.030)$, transfusion $(\mathrm{OR}=1.5, \mathrm{P}=0.020)$, and death $(\mathrm{OR}=4.5, \mathrm{P}=0.050)$. High $\mathrm{INR}$ was an independent risk factor for pneumonia $(\mathrm{OR}=8.7, \mathrm{P}=0.001)$, pulmonary embolism $(\mathrm{OR}=5.6, \mathrm{P}=0.021), \mathrm{DVT} / \mathrm{Thrombophlebitis}$ $(\mathrm{OR}=4.8, \mathrm{P}=0.011)$, Septic shock $(\mathrm{OR}=8.4, \mathrm{P}=0.048)$, and death $(\mathrm{OR}=9.8, \mathrm{P}=0.034)$. Bleeding disorder was an independent risk factor for organ space $\mathrm{SSI}(\mathrm{OR}=5.4, \mathrm{P}=0.01)$, pneumonia $(\mathrm{OR}=3.0$, $\mathrm{P}=0.023)$, and sepsis $(\mathrm{OR}=4.4, \mathrm{P}<0.001)$ (Table 1$)$.

Conclusion: Abnormal coagulation profile was found to be an independent predictor of morbidity and mortality in patients undergoing posterior lumbar fusion. As such, it should be considered in preoperative optimization and risk stratification.

287. 90-day Rehospitalization After Lumbar Spinal Fusion Surgery in New York State Between 2005 and 2014: A 10 Year Meta-analysis of the Statewide Planning and Research Cooperative System (SPARCS)

Gernot Lang, MD; Ali A. Baaj, MD; Mauricio J. Avila, MD; Jialin Mao; Wei-Chun Hsu; Roger Härtl, MD; Art Sedrakyan

Introduction: Re-admission is an important metric for quality in healthcare. Currently risk factors for readmission following spine surgery are under intense investigation. Minor evidence is available in terms of readmission rates specifically in patients undergoing lumbar spinal fusion surgery. The aim of this study is to analyze and evaluate unplanned readmission to hospital or emergency room within 90 days after primary lumbar spinal fusion surgery in New York State.

Methods: The New York Statewide Planning and Research Cooperative System (SPACRS) was utilized to capture patients undergoing primary lumbar spinal fusion surgery for degenerative pathologies of lumbar spine from 2005-2014. Temporal trend of 90-day readmission was assessed using Cochrane-Armitage test. Logistics regression was used to examine predictor associated with 90-day readmission.

Results: 87,004 patients were included into this large data study. The overall 90-day readmission rate was $24.8 \%$ (21581/87004). There was a decrease in same-day readmission $(\mathrm{p}<0.01)$ and a slight increase in $=1$-day readmission $(\mathrm{p}=0.16)$ in the 90 -day period following primary spinal fusion. The leading 90 -day complications associated with 90-day readmission were wound complications (3.7\%), wound infections (3.1\%) and pulmonary embolism $(2.7 \%)$. On a multivariable model, age (OR: 1.14 comparing $=75$ to $<35,95 \%$ CI: 1.04-1.25)), sex (OR comparing female to male: (1.16 (1.12-1.20)), length of stay (OR comparing >10d to 1-2d: 3.13 (2.89-3.38)), race (OR comparing African American to White: 1.48 (1.40-1.57)), insurance (OR comparing Medicaid to Medicare: 1.39 (1.29-1.49)), coronary artery disease (OR: 1.23 (1.16-1.30)), chronic pulmonary disease (OR: 1.24 (1.19-1.29)), congestive heart failure (OR:1.30 (1.14-1.47)), and diabetes (OR:1.27 (1.21-1.32) are significantly associated with 90-day readmission.

Conclusion: Age, sex, race, insurance, length of stay and comorbidities are major risk factors for 90-day readmission. Specific risk stratification should be conducted for patients at risk before undergoing spinal fusion surgery in order to prevent early readmission, improve quality of care and reduce health care expenditures.
288. Predictors of Readmission, Reoperation, and Venous Thromboembolic Complications After Spine Surgery: A Singleinstitution Experience with 6870 Consecutive Patients

Michael Brendan Cloney, BA; Ekamjeet Dhillon; Helena Roberts; Zachary A. Smith, MD; George R. Cybulski, MD; Tyler R. Koski, MD; Nader S. Dahdaleh, MD

Introduction: Readmission and reoperation are used as hospital and surgeon quality metrics. Venous thromboembolic events (VTE), including both deep venous thrombosis (DVT) and pulmonary embolism (PE), are a major cause of readmission, morbidity, and mortality after spine surgery. Specific procedural, perioperative, and patient characteristics may be associated with these outcomes.

Methods: We retrospectively examined records from 6870 consecutive spine surgeries at our institution. We collected data on patient demographics, surgery, hospital course, and 30-day rates of VTE, readmission, reoperation, and epidural hematoma requiring evacuation. Stepwise multivariable logistic regression was used to identify independent predictors of each outcome.

Results: Factors associated with VTE within 30 days of surgery include a history of VTE (OR 3.92 [1.83, 8.36], p < 0.001), EBL (OR 1.00 [1.00, 1.00], $\mathrm{p}=0.004)$, fracture (OR 5.42 [2.09, 14.05], $\mathrm{p}=0.001)$, history of PE (OR $4.04[1.22,13.42], \mathrm{p}=0.023)$, and transfusion (OR $2.26[1.07,4.77], \mathrm{p}=0.033)$. Factors associated with readmission were a history of PE (OR 3.27 [1.07, 9.97], $\mathrm{p}=0.038$ ), $\mathrm{PE}$ within 30 days $(\mathrm{OR} 8.07$ [2.26, 28.8], $\mathrm{p}=0.001)$, transfusion $(2.54$ $[1.55,4.17], \mathrm{p}<0.001)$, comorbid disease burden (OR 1.35 \{1.01, $1.80], \mathrm{p}=0.041$ ), and tumor surgery (OR $2.84[1.32,6.10], \mathrm{p}=0.007)$. Factors associated with reoperation were EBL (OR 1.00 [1.00, 1.00], $\mathrm{p}=0.008)$, transfusion (OR 3.86 [1.38, 10.79], $\mathrm{p}=0.01)$, and PE within 30 days (OR $6.05[1.03,35.62], \mathrm{p}=0.046)$. Only transfusion was associated with epidural hematoma within 30 days (OR 7.38 [1.37, 39.83], $\mathrm{p}=0.02$ ).

Conclusion: Transfusion and EBL are associated with numerous negative outcomes after spine surgery. Transfusion is an independent predictor of VTE, readmission, reoperation, and epidural hematoma requiring evacuation. Specific spinal pathologies as trauma/fractures were associated with specific negative outcomes.

\section{Preventing Promulgation of Inaccurate Complications}

John J. Knightly, MD; Scott A. Meyer, MD; Andrew Koslov; Sherry Ninni, RN, CPHQ, CCRN-K

Introduction: The increased use of billing data for quality benchmarking purposes in healthcare makes physician awareness of accurate coding and reporting more important than ever. Inaccurate diagnosis and procedural coding entered often by coding specialists can be a result of incomplete documentation by physicians or erroneously entered codes that do not accurately reflect the patient's condition or treatment. The use of codes for quality measures to benchmark physicians and institutions makes the fidelity of the data of great importance.

Methods: We evaluated 2063 consecutive patients who received neurosurgical care at our institution. Reports were obtained from our institutional database. Medical record review of each complication, including database assigned complications, AHRQ complications, observed to expected mortality deviations and 30-day readmissions, was performed. The complication was then identified as accurate, needing coding review or having incomplete documentation.

Results: Out of 2063 consecutive patients, 247 patients (12\%) had complications noted. After medical record review of those complications $51(21 \%)$ of the coded complications were identified as potentially miscoded and referred back to medical records for review. Of the 51 patients sent to be reassessed, a total of $34(67 \%)$ were corrected and recoded. 
Conclusion: Quality and safety measures are increasingly derived from coding databases. Knowledge of appropriate documentation principles as well as verification of coding accuracy can have a significant impact on quality and safety for benchmarking for physicians and institutions.

290. A Modified Risk Assessment and Prediction Tool For Predicting Discharge Destination Following Lumbar Surgery: A Multicenter Analysis of 937 Patients from the Quality Outcomes Database

\section{Jian Guan, MD; John J. Knightly, MD; Erica Fay Bisson, MD, MPH}

Introduction: Patients who are unable to return home following surgery represent a significant challenge to physicians and allied health professionals. No tools currently exist to help predict a patient's risk for non-home discharge following lumbar spine procedures. We modified a previously developed metric - the Risk Assessment and Prediction Tool (RAPT) - in order to help determine which patients may be at risk.

Methods: Using prospectively collected data from the Quality Outcomes Database at two high volume spine centers, we modified the RAPT score to match factors associated with discharge destination in the lumbar spine population. Chosen variables were age, gender, need for ambulation aids, insurance type, the presence of a motor deficit, a diagnosis of depression, and education level. Each variable was assigned a weighted value, and a modified RAPT score calculated for each patient. This score was then dichotomized based on the median score of 9 (range of 3-12). Chi-square analysis was performed to assess the strength of the correlation between the modified RAPT score and rate of discharge home.

Results: 937 patients met inclusion criteria. Of these $76(8.1 \%)$ were unable to discharge home postoperatively. Patients in the high risk (low modified RAPT) group were significantly more likely to discharge to a non-home destination than the low risk (high modified RAPT) group ( $13.3 \%$ vs. $2.3 \%$, p $<0.001)$.

Conclusion: The modified RAPT score may be a useful tool for determining which patients may be a higher risk for non-home discharge following lumbar spine surgery. Further evaluation of this tool using larger patient cohorts is warranted.

\section{Patient Expectations and Preferences in the Spinal Surgery Clinic}

Elizabeth Kuhn, MD; Matthew Christopher Davis, MD; Borna E. Tabibian, MD; Patrick R. Pritchard, MD

Introduction: Patient satisfaction, which reflects pain relief, restoration of function, realization of expectations, surgeon affability and patient engagement, is associated with improved clinical outcomes. Satisfaction can be improved when patient and surgeon expectations are aligned and patient preferences are met.

Methods: Patients presenting to clinic for spinal disease completed a questionnaire assessing demographics, pain, reason for visit, expectations and preferences. Variables were compared using chisquared tests or one-way analysis of variance to determine factors associated with patient expectations. New and return patients were compared utilizing matched pair t-tests.

Results: 240 patients were included. 141 patients $(60.7 \%)$ had at least some education beyond high school. New patient evaluation was the most common reason for evaluation $(26.6 \%)$, and pain relief was the most common chief concern (39.3\%). $20.7 \%$ of patients were seeing the surgeon for a second opinion and $45.3 \%$ had previously undergone spinal surgery by the neurosurgeon they were seeing. Patients preferred their surgeon wash their hands in the room instead of before entering $(\mathrm{p}<0.001)$, and wear professional attire over scrubs $(p<0.001)$. Patients believe their wait time will be longer than it should be $(p=0.002)$, spend longer in clinic than they should $(p=0.03)$, get less face-to-face time with their surgeon than they should $(\mathrm{p}<0.01)$, but also that the surgeon is not getting paid enough for the clinic visit $(p=0.02)$. While new patients felt they would not have as much faceto-face time with the surgeon as they should $(\mathrm{p}=0.030)$, there was no significant difference in anticipated versus acceptable face-to-face time among return patients $(\mathrm{p}=0.511)$.

Conclusion: In elective spine surgery, patients often seek treatment to improve quality of life and alleviate subjective symptoms. Understanding patient expectations is critical to ensure that patients and physicians are working toward similar goals. Targeted, multimodality educational interventions may improve patient satisfaction and engagement.

292. Using PROMIS Health Domains in Spine Surgery: An Evaluation of Responsiveness and Concurrent Validity

Taylor Elise Purvis, BA; S. Selverajah, MD, MPH; Elena Andreou; Brian J. Neuman, MD; Lee H. Riley, MD; Richard L. Skolasky, MD

Introduction: Patient-Reported Outcomes Measurement Information System (PROMIS) health domains may help assess quality of care and determine minimal important differences (MIDs) after surgery. We sought to determine the concurrent validity and responsiveness of the PROMIS domains and to establish MIDs for the PROMIS domains for patients with cervical or lumbar degenerative disease.

Methods: We included 368 adult patients undergoing surgery at one academic spine center from February 2015 through June 2016 for a lumbar or cervical spine degenerative condition. Concurrent validity was shown using correlation of PROMIS domains with legacy measures (Brief Pain Inventory; Generalized Anxiety Disorder 7-item scale [GAD-7]; Medical Outcome Study Short Form-12, version 2 [SF-12v2]; Neck Disability Index [NDI]; Oswestry Disability Index [ODI]; and Personal Health Questionnaire Depression Scale [PHQ8]) at the preoperative assessment. Responsiveness of PROMIS health domains was determined using the methods of Coyne et al. PROMIS MIDs were determined using distributional-based, cross-sectional anchor-based, and longitudinal anchor-based criteria (change from preoperative to postoperative, within 6 months).

Results: All 7 PROMIS domains showed moderate to strong correlations with NDI, ODI, SF-12v2 mental health, and pain interference and weak correlations with intensity of back/leg pain and neck/arm pain (except pain and neck pain $[\mathrm{r}=0.53, \mathrm{p}<0.05]$ ). PROMIS domains were well-correlated with GAD-7 and PHQ-8 except for physical function and satisfaction with social roles. The PROMIS pain domain showed strong responsiveness $(-0.95)$, whereas social role (0.76), physical function $(0.57)$, anxiety $(-0.54)$, sleep disturbance $(-0.59)$, and fatigue $(-0.60)$ showed moderate responsiveness. Depression $(-0.37)$ had weak responsiveness. Using the anchor-based method, the MIDs for each PROMIS domain were 45 points.

Conclusion: PROMIS health domains are valid tools for evaluating health-related quality of life in patients with cervical or lumbar degenerative disease and MIDs can be identified for assessing clinically meaningful change. 
293. Effect of Insurance Status on Patient-reported Outcomes for Lumbar Stenosis Patients Treated Surgically

Galal El-Sayed, MD; Samuel McClugage, MD; Esther Dupepe; Matthew Christopher Davis, MD; Mark N. Hadley, MD, FACS; Beverly C. Walters, MD, MSc, FRCS(C), FACS

Introduction: Evaluate the effect of payer status on outcomes in adult patients treated surgically for lumbar spinal stenosis.

Methods: Patients were evaluated as part of a quality outcomes database at a single institution. Data was obtained from 20122014 as part of a retrospective cohort. Outcome measures were evaluated at baseline, 3-months, and 12-months and analyzed in regard to payer status (private insurance versus Medicare/VA insurance).

Results: 100 patients were included in the study with outcome data for at least 3 months. At baseline, patients had similar scores for back/ leg pain based on a Visual-Analog Scale (VAS), Oswestry Disability Scale (ODI), and Euroqual-5D (EQ-5D). At 3 months, patients with government insurance reported more leg pain than those with private insurance (mean difference 1.26, $\mathrm{p}=0.051$ ) and lower EQ-5D (mean difference $0.11, \mathrm{p}<0.001$ ). At 12 months, patients with government insurance reported lower EQ-5D (mean difference 0.14, $\mathrm{p}<0.001$ ). There were no significant differences at 3 months or 12 months between groups for back pain ( $p=0.14$ and 0.42 ) or ODI $(p=0.19$ and 0.15 ). In paired outcomes analysis, patients in both groups showed improvement at 3 months and 12 months in all 4 functional outcomes compared to baseline $(\mathrm{p}<0.001)$

Conclusion: Patients with government insurance reported worse leg pain at 3 months, but this difference resolved at 12 months. At 3 months and 12 months, patients with government insurance reported worse quality of life than those with private insurance. This may be related to advanced age seen in the Medicare population or worse baseline ASA scores.

294. Barriers to the Use of Patient-reported Outcomes in Spine Care in Latin America: Rationale of the AOSLA Quality Assessment Registry

Asdrubal Falavigna, PhD; Alisson R. Teles, MD; Zoher Ghogawala, $M D, F A C S$

Introduction: The assessment of patient-reported outcomes (PRO) in spine care provides useful information for quality improvement, effectiveness, and comparative effectiveness. The objective of this study was to evaluate the perceptions about PROs among Latin America (LA) spine surgeons and to evaluate the barriers to implement its routine.

Methods: Internet-based survey to evaluate knowledge and perceptions on the use of PROs among members of AOSLA. The results of this survey supported the development of AOSLA Quality Assessment Registry.

Results: A total of 731 participants from 22 countries answered the electronic questionnaire, a response rate of $52.02 \%$. In general, more than $70 \%$ of participants agree that: PROs are useful to evaluate treatment outcomes, they help to monitor outcomes and burden of disease, they can benefit the patients, they facilitate physician-patient communication, and they are useful to compare the results of different centers. However, $36.8 \%$ of participants reported not using any PROs in their current activities. The main barriers to implementing PROs collection in routine practice were reported to be lack of time and structure (electronic database, assistants, etc) to perform this activity. However, when asked if they would use an electronic database to store and manage patient data, $87 \%$ of participants answered that they would use it routinely to monitor clinical outcomes of patients. Differences among countries were identified. The rationale of AOSLA Quality Assessment Registry is described in detail.

Conclusion: This survey identified the barriers to the use of PROs and clinical registries in spine care in LA. Strategies for overcoming these barriers should be addressed in order to fully implement a large clinical registry of spine care in LA.

295. The Effect of Prior Surgery on Hospital Consumer Assessment of Healthcare Providers and Systems (HCAHPS) Scores in Lumbar Spine Surgery Patients

Robert Winkelman, BA; Jay M. Levin, BA; Gabriel Alexander Smith, MD; Michael P. Steinmetz, MD; Thomas Mroz

Introduction: The Hospital Consumer Assessment of Healthcare Providers and Systems (HCAHPS) survey has made patient experience a key driver of quality and reimbursement for hospital systems and spine surgeons nationwide. Patients with a history of prior spine surgery have been linked to worse outcomes, such as longer hospital stays and higher complication rates, following additional surgery compared to patients with no prior history of spine surgery. The aim of this study was to explore the effect that prior spine surgery may have on the HCAHPS scores of lumbar surgery patients.

Methods: A retrospective cohort analysis was performed using the HCAHPS survey data of 405 patients who underwent lumbar decompression and/or fusion surgery at Cleveland Clinic Foundation's Main Campus location from 1/1/2013-12/31/2015. Prior surgery information was obtained via chart review and patients were stratified into three groups: no prior $(\mathrm{n}=258)$, one prior $(\mathrm{n}=96)$, and $>2$ prior surgeries $(\mathrm{n}=56)$. Pre-operative data for EuroQol 5 Dimensions (EQ-5D), Pain Disability Questionnaire (PDQ), and Visual Analog Score for back pain (VAS-BP) were also analyzed.

Results: A chi-squared analysis of pre-operative quality of life and disability scores demonstrated a significant difference across the three groups $(\mathrm{p}=0.02$ and $\mathrm{p}=0.002$, respectively). Analysis of HCAHPS responses also revealed a significant difference for questions pertaining doctor listening $(\mathrm{p}<0.01)$, doctor explanations $(\mathrm{p}=0.05)$, pain control $(\mathrm{p}=0.03)$, staff response to pain control $(p=0.01)$, and overall rating of the hospital $(p=0.02)$.

Conclusion: In our lumbar spine surgery cohort, history of prior lumbar spine surgery had a significant impact on the patient experience of care as measured by HCAHPS.

\section{The Association Between Patient Satisfaction and Clinical} Outcomes in Lumbar Spine Surgery

Jay M. Levin, BA; Robert Winkelman; Gabriel Alexander Smith, MD; Thomas Mroz; Michael P. Steinmetz, MD

Introduction: Under Value-Based Purchasing the patient experience of care - as measured by the Hospital Consumer Assessment of Healthcare Providers and Systems (HCAHPS) survey - is being used to determine hospital reimbursement. Current literature is inconsistent in finding an association between patient satisfaction and surgical outcomes. In our study, we evaluate whether there is an association between patient satisfaction on the HCAHPS survey and clinical outcomes following lumbar spine surgery.

Methods: This is a retrospective cohort study of patients undergoing lumbar spine surgery between 2013-2015. Patients were excluded if they had been diagnosed with spinal malignancy, scoliosis, or had less than one year of follow up. Patients who selected a 9 or 10 (top-box) Overall Hospital Rating (OHR) on HCAHPS were placed in the satisfied group, and the remaining patients comprised the unsatisfied group. The primary outcomes of this study include patient-reported health status measures such as EuroQol 5 Dimensions (EQ-5D), Pain Disability Questionnaire (PDQ), and Visual Analog Score for back pain (VAS-BP).

Results: Our study population consisted of 249 patients undergoing lumbar spine surgery. Of these, 197 patients selected a top-box OHR 
on the HCAHPS survey and were included in the satisfied group. 52 patients comprised the unsatisfied group. Almost all patient characteristics measured preoperatively were similar between the two groups, including clinical measures such as EQ-5D, PDQ and VAS$\mathrm{BP}$. The only preoperative characteristics that differed significantly were gender, a diagnosis of DDD, and a diagnosis of chronic renal failure. At one year follow-up, no statistically significant differences in EQ-5D, PDQ or VAS-BP were observed.

Conclusion: Both the satisfied and unsatisfied groups made similar improvements in EQ-5D, PDQ and VAS-BP measured at one year postoperatively. Therefore, high satisfaction was not associated with favorable clinical outcomes. These results suggest HCAHPS may not be a reliable indicator of quality care in lumbar spine surgery.

\section{The Impact of Depression on Patient Satisfaction in a Lumbar Spine Surgery Population}

Jay M. Levin, BA; Gabriel Alexander Smith, MD; Robert Winkelman; Thomas Mroz; Michael P. Steinmetz, MD

Introduction: Hospital Consumer Assessment of Healthcare Providers and Systems (HCAHPS) surveys are used to assess the quality of the patient experience, and directly influences reimbursement for hospital systems and spine surgeons nationwide. Untreated depression has been linked to worse functional outcomes in spine surgery. However, we aimed to elucidate if HCAHPS survey responses were different in depressed patients.

Methods: Prospectively collected functional outcome data including patient health questionnaire (PHQ-9), EuroQol 5 Dimensions (EQ-5D), Pain Disability Questionnaire (PDQ), and Visual Analog Score for back pain (VAS-BP) pre- and postoperatively on 400 patients was analyzed. Preoperative PHQ-9 scores of greater than or equal to 10 (moderate to severe depression) defined our depressed cohort of patients. HCAHPS responses were obtained for each individual, allowing for real-world analysis of outcomes in this population.

Results: In our 400 patient cohort, depressed patients were younger, female, earned less income, were on full disability, and had lower scores on EQ-5D, PDQ, and VAS-BP preoperatively. $81.4 \%$ of depressed patients felt doctors treated them with respect, compared to $91.0 \%$ of patients without depression $(\mathrm{p}=0.010)$. Also, depressed patients felt nurses treated them with less respect $(p=0.045)$ and that physicians did not listen to them as carefully $(\mathrm{p}=0.048)$. Multivariate regression analysis revealed that higher preoperative pain and disability had higher odds of patients feeling less respected and listened to by physicians. Moreover, patient smoking and lower median household income was associated with feeling less respected by physicians. Multivariate analysis also revealed that depression was an independent predictor of lower patient satisfaction with nursing response to their needs and with respect from nurses.

Conclusion: Patients with moderate to severe depression are less satisfied with the respect given by hospital staff while they are inpatient after spine surgery. Moreover, depressed patients are less likely to feel satisfied with physician listening and nursing response to their needs.

\section{The Impact of Preoperative Depression Diagnosis on} Surgical Outcomes for Lumbar Arthrodesis

Chloe O'Connell, BA; Anand Veeravagu, MD; Tej Deepak Azad, BA; Eli Johnson; Vaishali Mittal; Daniel Vail; John K. Ratliff, MD, FACS

Introduction: Previous research has found that depression is associated with poorer postoperative outcomes after lumbar spinal stenosis surgery, including increased postoperative pain and disability and increased patient dissatisfaction [1-4]. However, the use of national databases to conduct high-powered investigations of this relationship has been limited.

Methods: Using the Truven MarketScan database, we identified a cohort of 75,604 patients who underwent lumbar arthrodesis from 2006-2009. Covarying for comorbidities and demographic factors, we used linear and logistic regression to assess the association of depression diagnosis in the 6 months preceding surgery $(n=4,753)$ with complications, hospital readmission within 30 days of surgery, revision surgeries, cost of inpatient stay, and likelihood of discharge home. We also used poisson regression to determine the effect of depression on opiate prescriptions 18 months and 2 years postsurgery.

Results: Depression was associated with increased likelihood of complications $(\mathrm{OR}=1.19, \mathrm{p}<0.0001)$, readmissions $(\mathrm{OR}=$ $1.39, \mathrm{p}<0.0001)$, and revision surgeries $(\mathrm{OR}=1.39, \mathrm{p}<0.0001)$. Depression was also associated with a slightly increased log cost of hospitalization $(B=0.026, p=0.015)$, and length of stay $(B=0.17, p$ $=0.0003$ ). Compared with controls, depressed patients received 1.25 and 1.28 times as many opiate prescriptions 18 months and 2 years post-surgery, respectively (18 months: $\beta=0.22, \mathrm{p}<0.0001 ; 2$ years: $\beta=0.25, \mathrm{p}<0.0001)$.

Conclusion: These data suggest that depression diagnosis in the 6 months preceding lumbar arthrodesis negatively impacts outcomes in terms of complications, readmissions, revisions, inpatient stay length and cost, as well as number of opiate prescriptions up to two years following surgery. An understanding of the way psychiatric diagnoses impact surgical risk profiles may improve our ability to provide quality care for this population, by allowing for more accurate risk assessment based on patient characteristics.

\section{Psychological Burden of Musculoskeletal Diseases:} Investigation on Spine, Hip and Knee In-Hospital Patients

Bassel G. Diebo, MD; Cyrus M. Jalai, BA; Gregory W. Poorman; Qais Naziri, MD; Samantha R. Horn; Thomas Errico; Ashish Patel, MD; Virginie Lafage, PhD; Carl Paulino, MD; Peter G. Passias, MD

Introduction: Musculoskeletal disorders (MSK) are the leading cause of disability around the world. The psychological burden (PB) of these conditions experienced by patients and their families is of paramount importance. This study is investigation of the prevalence of psychological impairments in MSK in relation to other degenerative and chronic diseases.

Methods: Retrospective review of the National Inpatient Sample (NIS) from 2001-2012. ICD-9 coding identified MSK patients undergoing surgical treatment for spinal (SPINE) pathologies (cervical fusions for radiculopathy, myelopathy, or deformity [CERV], 2-3 level fusions for lumbar degenerative disease [DEGEN], and adult spinal deformity [ASD]), total hip arthroplasty (THA) and total knee arthroplasty (TKA). Patients hospitalized for chronic conditions, including cardiac disease (HEART), diabetes (DM), lung cancer (LUNG) were identified. Based on ICD-9-CM codes reported in the Diagnostic and Statistical Manual of Mental Disorders (DSM 5), PB was defined as incidence of any of the following disorders: Depressive, Anxiety, Obsessive-compulsive, Stress, Somatic symptoms, Sexual dysfunction, Substance-related and addictive, Delirium, and Personality.

Results: 5,263,447 discharges identified. On average, 27.8\% of MSK patients had psychological impairment. In details, MSK had the highest frequency of patients with sleep disorders, second highest with depression and anxiety and third highest with substance abuse. In SPINE, revision patients displayed higher PB in comparison to primary cases, however, patients undergoing revision joint surgeries had less PB). MSK patients with PB were younger (61.3 vs. 64.8 years), and $\mathrm{PB}$ was higher in females.

Conclusion: Prevalence of psychological impairments in patients undergoing surgery for certain musculoskeletal disorders is higher 
than admissions for diabetes, lung cancer, and heart diseases. Though these patients were found to be younger, data revealed associated increased length of stay and total hospital charges.

\section{Lumbar Spine Fusion: A RAND/UCLA Appropriateness Study}

Daniel Yavin, MD; Steven Casha, MD, PhD; Samuel Wiebe, MD, MSc; Thomas E. Feasby, MD; Jayna Holroyd-Leduc, MD; R. John Hurlbert, MD, PhD, FACS, FRCS(C); Hude Quan MD, PhD; Andrew Nataraj, MD; Garnette R. Sutherland, MD, FRCSC; John D. Bartleson, MD; Sean D. Christie, MD, FRCS(C); W. Jeptha Davenport, MD; Ted Findlay, DO; Ian G. Fleetwood, MD, BSc, FRCS $(C)$; Andrea Furlan, MD, PhD; Clare Naomi Gallagher, MD, PhD, FRCS (C); Karim Mukhida, MD; Jean Ouellet, MD, FRCSC1; John E. O'Toole, MD, MS; Charles A. Reitman, MD; Owen Williamson, MBBS; Perry Pawandeep Singh Dhaliwal, MD; Stephan Jean du Plessis; Nathalie Jette

Introduction: Regional rates of surgical fusion of the lumbar spine vary more than any other surgical procedure.

Methods: The RAND/UCLA Appropriateness Method was used to identify degenerative indications for lumbar fusion. A North American expert panel of 13 physicians independently rated clinical scenarios for lumbar fusion. Panelists rated 1296 scenarios from 1 to 9 for their appropriateness for lumbar fusion. A meeting was then convened in a modified Delphi process and scenarios were again rated. The resulting criteria were applied in 150 patients who underwent elective instrumented lumbar fusion.

Results: Of the 1296 final scenarios, fusion was appropriate in $133(10 \%)$, uncertain in $375(29 \%)$, and inappropriate in 735 (57\%). Disagreement occurred in the remaining 53 scenarios (4\%). Of the appropriate indications, spondylolisthesis accounted for $98(74 \%)$, spinal stenosis for $18(14 \%)$, spondylosis for $9(7 \%)$, and disc herniation for $8(6 \%)$. Appropriate fusion was associated with mechanical low back pain $(\mathrm{P}<0.001)$ and radiologic signs of instability or sagittal imbalance $(\mathrm{P}<0.001)$. Of the 150 operated patients, fusion was appropriate in $72(48 \%)$, uncertain in $70(47 \%)$, and inappropriate in $8(5 \%)$. In the 2 years after surgery, patients who underwent appropriate fusion required less cross-sectional imaging for persistent, worsening, or recurrent symptoms (adjusted hazard ratio [HR], 2.42; 95\% confidence interval [CI], 1.31 to 4.48; $\mathrm{P}<0.01$ ) and fewer spinal injections (adjusted HR, 2.53; 95\% CI, 1.29 to 4.96; $\mathrm{P}<0.01)$. There was, however, no significant difference between groups in the probability of reoperation $(\mathrm{P}=0.84)$, rehospitalization $(\mathrm{P}=0.50)$, or use of prescription pain medication $(\mathrm{P}=0.23)$.

Conclusion: Criteria for the appropriate use of lumbar fusion for degenerative indications were identified (web-based decision tool accessible at www.appropriatelumbarfusion.com/site). In operated patients, appropriate fusion was associated with reduced healthcare demands. The criteria will require further validation and regular revision.

\section{Low Cost Accelerometers in Spine Surgery: Preliminary} Results from a Prospective Validation Study

Paymon Rezaii; Allen Ho, MD; Arjun Vivek Pendharkar, MD; Eric S. Sussman, MD; John K. Ratliff, MD, FACS; Atman Desai, MD

Introduction: The increasing popularity of low-cost consumer based accelerometers (LCAs) have great potential to offer dynamic, real-time, longitudinal mobility data. This prospective study aims to validate LCA activity data and to explore the utility of LCAs in daily clinical practice to track patient activity following spine surgery.

Methods: We prospectively enrolled patients undergoing spine surgery into our clinical LCA study from May 2016 until September of 2016. All patients were given LCA devices preoperatively. Mobility data in the form of steps per day were extracted from the devices at various points during the study. The Oswestry Disability Index (ODI) and the Patient Health Questionnaire-2 (PHQ2) depression assessment were also assessed preoperatively and post-operatively. Pearson's correlation coefficients were calculated to examine correlation between LCA mobility data and QOL data.

Results: 23 patients were enrolled, of which 13 were female, with a mean age $52.6 \pm 17.1$ years. $65 \%$ of the patients had lumbar procedures, and $35 \%$ had cervical. $43 \%$ of the procedures involved a fusion. Average number of steps/day preoperatively was $5279 \pm 3014$ steps/day. Compared to average number of steps/day preop, postoperatively average number of steps/day decreased by $50 \%$ in the first week, $40 \%$ in the second week, and $21 \%$ in the first month. LCA data in terms of average number of steps were day were most well correlated to ODI, with a correlation coefficient of $r=0.56$ for preoperative steps per day and $r=0.52$ for change in average number of steps/day in the first month.

Conclusion: In our study, we saw expected improvements in post-operative mobility over time. Furthermore, LCA step data was well correlated with corresponding ODI scores. Thus, LCAs in spine surgery patients may potentially serve as a reliable, objective measure of longitudinal mobility in the perioperative population.

\section{Comparison of Patient Outcomes and Cost of Overlapping Versus Non-overlapping Spine Surgery}

Corinna Clio Zygourakis, MD; Saman Sizdahkhani; Malla K. Keefe, BS; Janelle Lee MD, PH; Praveen V. Mummaneni, MD; Christopher P. Ames, $M D$

Introduction: Overlapping surgery, also known as concurrent/ simultaneous surgery or "running two rooms", has recently gained significant media attention, but there is limited on its safety and efficacy. To date, there has been no analysis of overlapping surgery in the field of spine. The goal of our study was to compare the patient outcomes and cost of overlapping versus non-overlapping spine surgery.

Methods: We performed a retrospective review of 2,319 spine surgeries ( $\mathrm{n}=848$ overlapping; 1,471 non-overlapping) performed by three neurosurgery attendings from 2012-2015 at the University of California, San Francisco. Collected variables: patient age, gender, insurance, American Society of Anesthesiology score, severity of illness, risk of mortality, procedure type, surgeon, day of surgery, source of transfer, admission type, overlapping vs non-overlapping surgery $(=1$ minute of overlapping procedure time), MS-DRG, osteotomy, presence of another attending/fellow/resident. Statistics: Univariate, then multivariate mixed-effect models to evaluate the effect of the collected variables on the following outcomes: procedure time, estimated blood loss (EBL), length of stay, discharge status, 30-day mortality, 30-day unplanned readmission, unplanned return to OR, and total hospital cost.

Results: Urgent spine cases on inpatients and those transferred from other hospitals or emergency departments were more likely to be done in an overlapping fashion (all $\mathrm{p}<0.01$ ). After adjusting for patient demographics, clinical indicators, and procedure characteristics, overlapping surgeries had longer procedure times (estimate $=26.17 ; \mathrm{p}<0.001$ ) and lower rates of discharge to home (Odds Ratio $(\mathrm{OR})=0.65 ; \mathrm{p}<0.001)$, but equivalent rates of 30 -day mortality, readmission, return to OR, EBL, length of stay, and total hospital cost, as compared to non-overlapping surgeries (all $\mathrm{p}=\mathrm{ns}$ ).

Conclusion: Overlapping spine surgery may be performed safely by these three surgeons at our institution, although continued monitoring of patient outcomes is necessary. Overlapping surgery does not lead to higher hospital costs. 
303. Geographic and Hospital Variation in Cost of Lumbar Laminectomy and Lumbar Fusion for Degenerative Conditions

Corinna Clio Zygourakis, MD; Caterina Liu; Glenn Wakam, MD; Chris Moriates, MD; Christy Boscardin; Adams Dudley, MD; Ralph Gonzales, MD; John K. Ratliff, MD, FACS; Praveen V. Mummaneni, MD; Christopher P. Ames, MD

Introduction: Spinal surgery costs vary significantly across hospitals and regions, but there is insufficient understanding of what drives this variation. The goal of this study is to examine the factors underlying the cost variation for lumbar laminectomy/discectomy and lumbar fusions.

Methods: We obtained patient information (age, gender, race, severity of illness, risk of mortality, population of county of residence, median zipcode income, insurance status, elective vs non-elective admission, length of stay) and hospital data (region, hospital type, bed size, wage index) for all patients who underwent lumbar laminectomy/discectomy $(n=181,267)$ or lumbar fusions $(n=433,364)$ for degenerative conditions in the 2001-2013 National Inpatient Sample database. We performed unadjusted and adjusted analyses to determine which factors affect cost.

Results: Mean costs for lumbar laminectomy/discectomy and lumbar fusion increased from $\$ 8,316$ and $\$ 21,473$ in 2001 (in inflation-adjusted 2013 dollars), to $\$ 11,405$ and $\$ 29,438$, respectively, in 2013. There was significant regional variation in cost, with the West being the most expensive region across all years and showing the steepest increase in cost over time. After adjusting for patient and hospital factors, the West was $23 \%$ more expensive than the Northeast for lumbar laminectomy/discectomy, and $25 \%$ more expensive than the Northeast for lumbar fusion $(p<0.01)$. Higher wage index, smaller hospital bed size, and rural/urban non-teaching hospital type were also associated with higher cost for lumbar laminectomy/discectomy and fusion $(\mathrm{p}<0.01)$.

Conclusion: After adjusting for patient factors and wage index, the Western region, hospitals with smaller bed sizes, and rural/urban non-teaching hospitals were associated with higher costs for lumbar laminectomy/discectomy and lumbar fusion.

\section{Four-year Cost Analysis for the Spinal Laminectomy Versus Instrumented Pedicle Screw (SLIP) Trial}

Vijay Ravindra, MD, MSPH; Robert G. Whitmore, MD; Jill Curran, MS; Subu N. Magge, MD; Zoher Ghogawala, MD, FACS

Introduction: The Spinal Laminectomy versus Instrumented Pedicle Screw (SLIP) trial demonstrated that the addition of lumbar fusion to laminectomy was associated with improved health-related quality of life versus laminectomy alone. Additionally, there was a reoperation rate of $14 \%$ in the fusion group versus $34 \%$ in the decompression-alone group; we performed a four-year cost analysis.

Methods: The participants were enrolled in a randomized, controlled clinical trial comparing laminectomy versus laminectomy with fusion for grade I degenerative spondylolisthesis (SLIP) between 2002-2009. Direct costs from the SPORT trial, which were derived from 2004 Medicare diagnosis related group payments, were applied to the SLIP cohort and inflation adjusted for 2015. Short Form-6D (SF-6D) utility scores were computed from the SF-36, and gains in Quality-Adjusted Life-Years (QALYs) were calculated by taking the area under the utility curve relative to the baseline.

Results: Thirty-five patients underwent laminectomy and 31 were assigned to undergo laminectomy plus fusion. Fourteen patients (21\%) underwent re-operation: 12 patients $(34 \%)$ in the laminectomy group and $4(14 \%)$ in the laminectomy with fusion group $(\mathrm{p}=0.05)$. Total costs, including reoperations, were lower for laminectomy alone compared to fusion $(\$ 55,193.88$ vs. $\$ 71,904.14)$. Patients receiving fusion showed greater gain in QALYs at 4 years compared to laminectomy alone (0.64 vs. $0.39, \mathrm{p}=0.086)$. Cost/QALY ratios were comparable for each procedure (fusion: $\$ 112,350.22$ vs. laminectomy: $\$ 141,522.77$ ). Incremental cost-effectiveness ratio (ICER) of fusion compared to laminectomy alone was $\$ 68,841.04$ well below the societal willingness-to-pay threshold of $\$ 100,000$.

Conclusion: The four year cost analysis demonstrates that laminectomy with fusion is associated with greater gain in QALYs at 4 years compared to laminectomy alone and is cost-effective for Grade I degenerative lumbar spondylolisthesis given the four-year re-operation rate in both groups; further analysis using exact cost data from the Medicare database is necessary.

\begin{abstract}
305. Comparison of Anterior Cervical Discectomy and Fusion to Posterior Cervical Foraminotomy for Cervical Radiculopathy: Utilization, Costs and Adverse Events 2003-2014
\end{abstract}

Christopher D. Witiw, MD; Fabrice Smieliauskas, PhD; John E. O'Toole, MD, MS; Michael G. Fehlings, MD, PhD, FRCS(C), FACS; Richard G. Fessler, MD, PhD

Introduction: Surgery for cervical radiculopathy is typically approached by either anterior cervical discectomy and fusion (ACDF) or posterior cervical foraminotomy (PCF). ACDF is more common, however recent single center studies suggest comparable efficacy and significant cost savings with PCF in appropriately selected patients. $(1,2)$ To compare ACDF and PCF in terms of approach related morbidity and costs from a national perspective.

Methods: A sequential algorithm was used to identify those undergoing ACDF or PCF for cervical radiculopathy from the Truven Health MarketScan Research Database spanning 2003 to 2014. Outcomes consisted of mortality, adverse events and readmission to hospital over a 30-day post-intervention horizon. Hospital length of stay and total payments to the health provider by the individual, a third party payer or Medicare were assessed. Propensity score matching was used to balance groups on baseline covariates.

Results: The PCF cohort comprised 4,851 subjects and the ACDF cohort included 46,147 . Time in hospital was 0.28 days [95\% CI: 0.25 , $0.31, \mathrm{p}<0.001]$ shorter for the PCF cohort. A significantly greater proportion of PCF cases were discharged on the same day $(70.6 \%$ vs. $46.1 \%$; $\mathrm{p}<0.001)$. Mortality $(0.1 / 1000, \mathrm{p}=0.012)$, vascular injury $(0.2 / 1000, p=0.001)$, post-operative dysphagia/dysphonia $(14.5 / 1000$, $\mathrm{p}<0.001)$, cutaneous cerebrospinal fluid leak $(0.2 / 1000, \mathrm{p}=0.002)$ and deep venous thrombosis $(0.9 / 1000, p=0.013)$ occurred more frequency in the ACDF cohort. Conversely, wound infections (14.6/1000, $\mathrm{p}<0.001)$ and 30-day readmissions to hospital $(9.8 / 1000, \mathrm{p}<0.001)$ were significantly more frequent in the PCF cohort. Unadjusted total payments for PCF were $\$ 15,281 \pm 12,225$ and $\$ 26,849 \pm 16,309$ for ACDF. Matched difference was -\$11,726 [95\% CI: -\$12,221, - $\$ 11,232$, $\mathrm{p}<0.001]$ over a 30 day horizon; favoring PCF.

Conclusion: These findings, from a national sample of commercially-insured patients, suggest an opportunity for value improvement in the management of cervical radiculopathy and indicate a need for large scale comparative study of clinical outcomes and costs. 306. Drivers of 90-day Cost After Anterior Cervical Discectomy
and Fusion for Degenerative Disease

Ahilan Sivaganesan, MD; Silky Chotai, MD; Matthew J. McGirt, MD; Clinton J. Devin, MD

Introduction: Under the Affordable Care Act, the bundling of payments for elective spine surgery is imminent. Variability in costs associated with the 90-day global period will affect neurosurgeons' ability to stay solvent. We therefore set out to determine the drivers 
of variability in 90-day cost for elective anterior cervical discectomy and fusion (ACDF).

Methods: A total of 445 patients undergoing elective ACDF were included in the study. Hospital discharge and billing records were collected in a prospective web-based registry. Total cost during the 90-day global period was derived from the diagnosis-related group (DRG) code (hospital fee), CPT code (surgeon fee), visits to providers (such chiropractors, PT, and OT), ER visits, readmissions, imaging, and medications. All cost data were adjusted based on Medicare national allowable payment amounts. Univariate (Figures 1 and 2) and multivariate linear regression modeling was performed for total 90-day cost which incorporated an array of pre-operative, operative and post-operative variables.

Results: The mean 90-day direct cost was $\$ 17685 \pm \$ 5731$ (median $\$ 16,394$; range $\$ 9,494$ to $\$ 62,590$ ). In a multivariate linear regression model, the duration of surgery, number of levels, length of stay, history of anticoagulant use, post-discharge resource utilization (imaging), complications, and readmissions were significant contributors to cost. The regression equation is as follows: total cost $=\$ 6309+\$ 1494$ (number of levels) $+\$ 32$ (length of surgery in min) $+\$ 5796$ (preoperative anticoagulation) + \$2263 (length of stay) + $\$ 4890$ (complications) + \$9344 (readmission) + \$758 (each x-ray post-discharge). The model performance as measured by R-squared was 0.601 .

Conclusion: We present a model which highlights drivers of 90-day cost following elective ACDF. Our model explains $60 \%$ of the variation in cost. Risk adjustment, based on factors included in our model, must be a key component of bundled payment initiatives if they are to be sustainable and effective in the long-term.

307. Anterior Cervical Diskectomy and Fusion (ACDF) in the Ambulatory Care Setting: Defining its Value Across the Acute and Post-acute Care Episode

Matthew J. McGirt, MD; Saniya S. Godil, MBBS; Tim E. Adamson, MD; E. Hunter Dyer, MD; Silky Chotai, MD; Anthony L. Asher, MD, FACS; Domagoj Coric, MD

Introduction: In the era of current healthcare reforms, all stakeholders have adopted value-based purchasing strategies to shift care toward higher benefit and lower cost treatment approaches. Degenerative spine disease is highly prevalent and its surgical intervention costly. In this study, we set out to quantify the potential cost savings and patient-centered benefits associated with performing ACDF in an ambulatory surgery center versus inpatient hospital setting.

Methods: 112 consecutive cases of one or two-level anterior cervical diskectomy and fusion(ACDF) performed at two centers were prospectively enrolled into a common registry. Data was collected on patient demographics, operative details and peri-operative and 90-day morbidity. 90-day morbidity, return to work, and 3-month patient reported outcomes were prospectively assessed. Direct costs were estimated from resource utilization via macro-costing with private pay estimated as $1.7 \mathrm{x}$ Medicare fee schedule. Indirect costs were calculated from lost work productivity using standard human capital approach.

Results: 53 outpatient ACDF and 59 inpatient ACDF patients were included. Cohorts were similar at baseline. 90-day surgical morbidity was similar between outpatient vs inpatient cohorts: 30 -day readmission $(0.0 \%$ vs. $1.7 \% ; \mathrm{p}=0.34)$, 90 -day readmission $(0.0 \%$ vs. $1.7 \% ; \mathrm{p}=0.34)$, DVT $(0.0 \%$ vs. $1.7 \% ; \mathrm{p}=0.34)$, dysphagia requiring $\mathrm{NPO} / \mathrm{NG}$ tube $(0.0 \%$ vs. $1.7 \% ; \mathrm{p}=0.34)$ and neck hematoma $(0.0 \%$ vs. $1.7 \% ; p=0.34)$,Table 1 . Improvement in three-month pain, disability, QOL, and return to work were also similar between two cohorts, Figures $1 \& 2$. Mean total 3-month cost per patient was significantly reduced in the outpatient vs. inpatient surgery cohort ( $\$ 20,043$ vs. $\$ 27,123 ; p<0.001)$ with similar QALY-gained,Table 2.
Conclusion: During the acute care and post-acute care episode, the outpatient ambulatory care versus inpatient hospital setting was associated with significant cost savings without a compromise in safety or clinical effectiveness for ACDF. From a patient, payer, purchaser, and societal perspective, the ambulatory surgery center setting offers superior value and can lead to cost savings of over $\$ 7,000$ per patient.

308. Posterior Micro-Endoscopic Discectomy vs. ACDF for Single-level Radiculopathy: Comparative Effectiveness and CostUtility Analysis

Matthew J. McGirt, MD; E. Hunter Dyer, MD; Domagoj Coric, MD; Silky Chotai, MD; Anthony L. Asher, MD, FACS; Tim E. Adamson, $M D$

Introduction: Cervical radiculopathy remains highly prevalent and costly in the U.S. healthcare system. While ACDF has remained the most popular surgical treatment modality, minimally invasive advancements such as posterior micro-endoscopic discectomy/ foraminotomy(pMED) has emerged as a motion preserving and less invasive alternative. To date, the comparative-effectiveness and costeffectiveness of pMED vs. ACDF remains unclear.

Methods: Patients undergoing surgery for single-level radiculopathy without myelopathy resulting from foraminal stenosis or foraminal disc herniation without instability over a one-year period were prospectively enrolled into an institutional database. Baseline, post-operative 3-months, and 12-months VAS-Arm and Neck, NDI, EQ-5D, and return to work(RTW) status were collected. Direct healthcare cost(payer perspective) and indirect cost (work-day losses multiplied by median gross-of-tax wage and benefits rate) was assessed.

Results: Total 20 ACDF and 28 pMED patients were identified. Baseline demographics, symptomatology, and co-morbidities were similar between the cohorts. For pMED vs. ACDF, mean length of surgery $(48.1 \pm 20.0$ vs. $69.9 \pm 11.6$ minutes, $\mathrm{p}<0.0001)$ and estimated blood loss $(20.3 \pm 9.3$ vs. $31.8 \pm 15.4 \mathrm{~mL}, \mathrm{p}=0.04)$ was reduced. There was no 90-day morbidity or re-admission for either cohort. One(3.6\%) pMED patient required a subsequent ACDF; no patients in the ACDF cohort required re-operation by one-year. $\mathrm{pMED}$ and $\mathrm{ACDF}$ cohorts demonstrated similar improvement in arm-VAS(3.1 vs. 2.6, $\mathrm{p}=0.66)$, neck-VAS(2.0 vs. $3.2, \mathrm{p}=0.24), \operatorname{NDI}(9.0$ vs. $6.8, \mathrm{p}=0.24)$, and EQ-5D(0.17 vs. $0.15, \mathrm{p}=0.82)$. Ability to RTW(93.8\% vs. $94.1 \%, \mathrm{p}=1.0)$ and median time to RTW(3.7[0.9-8.1] vs. 3.6[2.1-8.5] weeks, $\mathrm{p}=0.85)$ were similar,Fig 1 . pMED was associated with significantly reduced direct $\operatorname{cost}(p>0.001)$ but similar indirect $\operatorname{cost}(p=0.43)$, resulting in an average total cost savings of $\$ 7,689(\mathrm{p}<0.01)$ per case with similar QALY-gain (0.17 vs. $0.15, \mathrm{p}=0.82$ ).

Conclusion: For single-level unilateral-radiculopathy resulting from foraminal stenosis or lateral disc herniation without segmental instability, $\mathrm{pMED}$ was equivalent to ACDF in safety and effectiveness. pMED represents a minimally invasive, motion preserving alternative to select patients with cervical radiculopathy without the need for implant costs with concomitant significant cost saving.

309. Navigating Risk in a Capitated or Bundled Payment Model for Spine Surgery: Introduction of the Carolina - Semmes Prediction Tool

Matthew J. McGirt, MD; Scott L. Parker, MD; Silky Chotai, MD; Deborah Pfortmiller; Jeffrey M. Sorenson, MD; Kevin T. Foley, MD, FACS; Anthony L. Asher, MD, FACS

Introduction: Extended length of hospital stay(LOS), unplanned hospital readmission, and need for inpatient rehabilitation following 
spine surgery contribute significantly to variation in surgical healthcare cost. As novel payment models shift, the risk of cost over runs from payers to providers, understanding patient-level risk of these events is critical. We set out to develop a grading scale that stratifies risk of these costly events after elective surgery for degenerative lumbar pathologies.

Methods: 6,921 cases prospectively enrolled into the QOD registry were queried (elective 1-3 level lumbar surgery for degenerative pathology). The association between pre-operative patient variables and extended $\operatorname{LOS}(=7$ days), discharge status (inpatient facility vs. home), and 90-day hospital readmission were assessed by step-wise multivariate logistic regression. Carolina-Semmes grading scale was constructed using the independent predictors for LOS (0-8 points), discharge to inpatient facility (0-10 points), and 90-day re-admission $(0-8)$, its performance was assessed in the QOD dataset and then confirmed separately after applying to the Carolina Neurosurgery \& Spine Associates[CNSA] and Semmes-Murphy Clinic sites.

Results: $290(4.2 \%)$ patients required extended LOS, 654 (9.4\%) required inpatient facility rehab, and 474 (6.8\%) 90-day hospital readmission. Variables independently associated with these unplanned events in multivariate analysis are summarized in Table 1. Increasing point totals in the Carolina-Semmes scale effectively stratified the incidence of extended LOS, discharge to facility, and re-admission in both the aggregate QOD dataset(Fig 1) and when subsequently applied to two practice groups(Fig 2).

Conclusion: For patients undergoing first time elective 1-3 level degenerative lumbar spine surgery, we introduce the CarolinaSemmes grading scale that effectively stratifies risk of prolonged hospital stay, need for post-discharge inpatient facility care, and 90 -day hospital readmission. This scale may be helpful in identifying high-risk patients who may benefit from preventative health services strategies and education as well as help structure capitated/bundled care contracts to minimize risk on the provider.

\section{Genetic Influences of Lumbar Disk Herniation in Young Patients}

Travis Fulton; C. Rory Goodwin, MD, PhD; A. Karim Ahmed; Daniel M. Sciubba, MD; Nicholas Theodore, $M D$

Introduction: Intervertebral disk disease (IVDD) is one of the most common causes of low back pain and can substantially impact patient quality of life. Lumbar disk herniation has traditionally thought to be attributed to natural 'wear and tear' and mechanical insult. However, studies in the past two decades, however, have demonstrated the role for genetic influence instead of solely environmental factors. Intervertebral disk disease (IVDD) is one of the most common causes of low back pain and can substantially impact patient quality of life. Lumbar disk herniation has traditionally thought to be attributed to natural 'wear and tear' and mechanical insult. However, studies in the past two decades, however, have demonstrated the role for genetic influence instead of solely environmental factors.

Methods: A cohort of patients with definitively diagnosed lumbar intervertebral disk disease was compiled. The cohort was younger than the average age of presentation and excluded based on environmental risk factors for IVDD. A genome-wide association study (GWAS) was performed to characterize the genetic influences that may predispose IVDD.

Results: Missense mutations in collagen encoding genes were observed in 13 out of 15 patients in the cohort with intervertebral disk disease. Moreover, the odds ratios of key variants in Collagen 9A2 (COL9A2) and Collagen 11A1 (COL11A1) were greater than 1. The IVDD cohort also demonstrated statistical significance for 2 variants in the gene encoding for aggrecan which facilitates load-bearing properties in the cartilaginous end plate.

Conclusion: These results support the previously published work for collagen variants as a genetic risk factor for intervertebral disk disease. However, the present study sheds new light on the role for variants in aggrecan, which sustains the cartilaginous end plate. Genetic predisposition to IVDD, therefore, may be a multimodal combination of mutations in the nucleus pulposus, annulus fibrosus, as well as cartilaginous end plates.

\section{Epidural Corticosteroids Infiltrations for Lumbar Stenosis: A Prospective Study}

Majd G. El Hajj Moussa, MD; Charbel Tawk, MD; Georges Nohra; Souhail Chamandi; Fadi Hoyek, MD; Jean-Claude Lahoud, MD, $\mathrm{PhD}$

Introduction: Lumbar stenosis (LS) is a major cause of lower back pain and functional disability. Major symptoms are low back pain and radicular claudication. Medical therapeutic options include painkillers, NSAIDS, Steroids, antiepileptic drugs and epidural infiltrations. Surgery is indicated when medical treatment fails.

Methods: This prospective study was conducted on 60 patients presenting to our institution. Isolated LS was suspected after physical examination and was confirmed by MRI. All our patients were treated with NSAIDS and antiepileptic drugs (Gabapentin $800 \mathrm{mg}$ daily) for 6 weeks. 7 patients had laminectomy during the year of follow-up and were excluded from the statistical analysis; 53 patients were followed-up for 1 year (88.3\%). The treatment protocol consisted of three interlaminar epidural infiltrations under fluoroscopic guidance of $80 \mathrm{mg}$ Methylprednisolone and $100 \mathrm{mg}$ of Lidocaine administered at 2-weeks interval at the level of the stenosis. Evaluation was done using 4 scales: - Visual Numerical Scale (VNS) - Roland5 Point Scale (R5PS) - Walking distance (WD) (0 to 4) - Patient satisfaction scale (PS).

Results: Mean VNS: D0: 7,27 (4-10), D15: 3,13 (0,5 to 6), 1y: 3,45 (1 to 6) (p <0.0001) Mean R5PS: D0:4,08 (3 to 5), D15: 1,85 (0 to 3), 1y: 1,83 (1 to 4) ( $\mathrm{p}<0.0001)$ Mean WD: D0: 1,85, D15: 3,34, 1y: $3,34(p<0.0001) 50 \%$ amelioration of VNS: D15: $71.1 \%, 1 y: 67.9 \%$ $(p<0.0001) .50 \%$ amelioration of R5PS: D15: $75.5 \%$, 1y: $75.5 \%$ $(\mathrm{p}<0.0001) 50 \%$ amelioration of WD: D15: $50.9 \%$, 1y: $52.8 \%$ PS: Very good and good results $=65 \%$, Average and bad results $=35 \%$.

Conclusion: Based on our results, ESI are efficient in LS. Our study correlates with many results in the literature although few studies were conducted exclusively on LS. We attributed the efficacy of steroids to their probable ability to block the nociceptive pathways and to their effect on prostaglandins As an alternative for medical treatment, ESI could be a promising option before indicating surgery in LS, or in surgery contraindications.

\section{The Clivoaxial Angle is Associated With Development of Adjacent Segment Pathology After Craniocervical Fusion}

Shashank Gandhi; Salvatore Insinga, DO; Timothy G. White, BA; Ahmad Latefi, DO; Harold L. Rekate, MD

Introduction: Reduction by craniocervical fusion is a treatment option for craniovertebral instability and basilar invagination. The skull is placed into extension, anteriorly translating the odontoid, alleviating brainstem compression. We hypothesize that an increase in the degree of extension, measured by the clivoaxial angle, is associated with a higher risk of cervical developing adjacent segment pathology.

Methods: A retrospective, single institution analysis of all craniocervical decompression and occiput-C2 or occiput-C3 fusions from 2012-2015. Radiographic measurements were made from preand postoperative MRI/CT/X-rays: clivoaxial angle (CXA), GrabbOakes line $(\mathrm{pBC} 2)$, cervical kyphosis, cervical disc degeneration, and subluxation. Patients were grouped based on the development of 
adjacent segment pathology and statistical analysis with t-test, logistic regression, and Fisher's exact test was conducted.

Results: 83 patients underwent CCF. 70 met inclusion criteria for analysis. Ten (14.3\%) patients developed ASP specifically kyphosis. $3(4.3 \%)$ had cervical subluxation, $2(2.9 \%)$ of which required surgery. There was no significant difference for preoperative CXA between the non-ASP and ASP groups (127.2 \pm 6.8 versus $126.9 \pm 10.3$ degrees; $\mathrm{p}=0.901)$. Postoperative mean CXA were smaller in the non-ASP group (144.6 \pm 7.9 versus $156.5 \pm 8.7$ degrees; $\mathrm{p}=0.0001)$. Mean preoperative $\mathrm{pBC} 2$ was not different $(9 \pm 1.2$ versus $9.4 \pm 1.5 \mathrm{~mm}$; $\mathrm{p}=0.363$.). The change in CXA was significantly different in nonASP and ASP groups (17.4 \pm 8.8 versus $29.6 \pm 9.5$ degrees; $\mathrm{p}=0.0002$ ). Logistic regression analysis revealed that a large CXA was a risk factor for ASP $(\mathrm{OR}=1.2 ; \mathrm{p}=0.002)$. At CXAs of 145 and 155 the probability of ASP was $5.6 \%$ and $28.6 \%$, with $\mathrm{OR}=6.7$ for every 10 -degree increase in CXA. The postoperative $\mathrm{pBC} 2$ was larger in the non-ASP group $(6.2 \pm 1.4$ versus $5.3 \pm 0.8 \mathrm{~mm} ; \mathrm{p}=0.045)$.

Conclusion: Patients with craniovertebral instability and basilar invagination who undergo CCF with a large CXA have an increased risk of ASP mostly kyphosis. Surgeons must balance increasing the CXA to reduce brainstem compression with the increased risk of developing adjacent segment pathology.

\section{The Effect of Smoking Status on Instrumentation Success After rhBMP-2 Supplemented Lumbar Fusion Constructs}

Mohamed Macki, MD, BA; Sbaa Syeda, BS; Kenan Rajjoub, BA; Panagiotis Kerezoudis; Ali Bydon, MD; Jean-Paul Wolinsky, MD; Timothy F. Witham, BS, MD; Daniel M. Sciubba, MD; Mohamad Bydon, MD; Ziya L. Gokaslan, MD

Introduction: The primary objective of this study is to examine the effects smoking status on rhBMP-2 supplementation in spinal fusion constructs.

Methods: Patient records were retrospectively reviewed for a consecutive set of patients who underwent first-time posterolateral, instrumented fusion of the lumbar spine for degenerative spinal disease. All operations included arthrodesis supplementation with rhBMP-2. All patients were followed for at least two years. The primary endpoint of this study was reoperation for pseudarthrosis, instrumentation failure, and/or adjacent segment disease. Following rigorous sensitivity analysis, measure of association was calculated with a multivariable logistic regression controlling for smoking, age, and number of spinal levels fused.

Results: Of the 110 patients in the study population, 82 $(74.6 \%)$ were non-smokers and $28(25.5 \%)$ were smokers. Among perioperative predictors, smokers were younger in age (53.9 \pm 9.6 vs $61.1 \pm 13.1, \mathrm{p}=0.008$ ) and had shorter length on inpatient hospital stay ( $4.1 \pm 1.8$ vs $5.3 \pm 3.0, p=0.039)$. After a mean follow up of 59 months, the $32 \%$ incidence of reoperation for pseudarthrosis, instrumentation failure, and/or adjacent segment among smokers was statistically significantly higher than the $13.4 \%$ incidence in non-smokers $(p=0.027)$. Following multivariable logistic regression, the odds of reoperation among smokers was 4.75 times higher than non-smoker $(\mathrm{p}=0.009,95 \%$ Confidence Interval [1.48 15.24]).

Conclusion: While rhBMP-2 supplements arthrodesis of instrumented lumbar fusion constructs, smoking status ascertains the strongest predictor of reoperation for pseudarthrosis, instrumentation failure, and/or adjacent segment.
314. Micro vs. Macrodiscectomy: Does Use of the Microscope Reduce Complication Rates?

Meghan Murphy, MD; Jeffrey Hakim, PhD; Panagiotis Kerezoudis; Jennifer Grauberger; Mohammed Ali Alvi, MD; Daniel S. Ubl, BA; Elizabeth B. Habermann, PhD; Mohamad Bydon, MD

Introduction: A single level discectomy is one of the most common procedures performed by spine surgeons. While some practitioners utilize the microscope, others do not. We postulate improved visualization with an intraoperative microscope decreases complications and inferior outcomes.

Methods: A multicenter surgical registry was utilized for this retrospective cohort analysis. Patients with degenerative spinal diagnoses undergoing elective single level discectomies from 20102014 were included. Univariate analysis was performed comparing demographics, patient characteristics, operative data, and outcomes for discectomies performed with and without a microscope. Multivariable logistic regression analysis was then applied to compare outcomes of micro- and macrodiscectomies.

Results: Query of the registry yielded 23,583 patients meeting inclusion criteria. On univariate analysis the microscope was used in a greater proportion of the oldest age group as well as Hispanic white patients. Patients with any functional dependency, history of congestive heart failure, chronic corticosteroid use, or anemia (hematocrit $<35 \%$ ) also had greater proportions of microdiscectomies. Thoracic region discectomies more frequently involved use of the microscope than cervical or lumbar discectomies (25.0\% vs. $16.4 \%$ and $13.0 \%$, respectively, $\mathrm{p}<0.001$ ). Median operative time (IQR) was increased in microscope cases $[80$ minutes $(60,108)$ vs. 74 minutes $(54,102), \mathrm{p}<0.001]$. Of the patients that required reoperation within 30 days, $2.5 \%$ of them had undergone a microdiscectomy compared to $1.9 \%$ who had undergone a macrodiscectomy, $p=0.044$. On multivariable analysis, microdiscectomies were more likely to have an operative time in the top quartile of discectomy operative times, $=103$ minutes (OR 1.256, 95\% CI 1.151-1.371, $\mathrm{p}<0.001$ ). In regards to other multivariable outcome models for any complication, surgical site infection, dural tears, reoperation, and readmission, no significant association with microdiscectomy was found.

Conclusion: The use of the microscope was found to significantly increase the odds of longer operative time, but not influence rates of postoperative complications. Thus, without evidence from this study that the microscope decreases complications, the use of the microscope should be at the surgeon's discretion, validating the use of both macro and micro approaches to discectomy as acceptable standards of care.

\section{Extraforaminal Disk Herniations: What is the Best Approach? A Systematic Review and Meta-analysis}

Oluwaseun Akinduro, MD; Panagiotis Kerezoudis; Jamachi Eluchie; Jang W. Yoon, MD, MS, BS; Mohamad Bydon, MD

Introduction: Far lateral disk herniation accounts for about 3-11\% of all disk herniations. Due to the heterogeneity of spinal procedures, especially operations for disk herniation, there have been no clear studies demonstrating superiority of one surgical approach over others.

Methods: We performed a systematic review and meta-analysis of all PubMed and EMBASE for literature available for extra-foraminal disk herniation. Articles were limited to full text articles involving humans published in English, which resulted in 205 articles, which were reviewed for inclusion. We excluded studies with less than 8 patients, thoracic disk herniation, imaging studies, cadaveric studies, articles in which the extraforaminal patients were not analyzed separately, and articles with only paramedian or intraforaminal herniation patients. 
Results: The difference in rate of complications for the open surgery group (open $\&$ microscopic) versus the minimally invasive group (tubular microscopic, tubular endoscopic \& purely endoscopic) was found to be statistically significant favoring the minimally invasive group [6.7\% versus $2.4 \%(\mathrm{P}=0.0002)]$. The difference in reoperation rate was found to be statistically significant favoring the open group [2.5\% versus $5.4 \% \mathrm{P}=(0.0045)]$. The difference in MacNabs criteria for the open cohort versus the minimally invasive cohort was found to not be statistically significant, but favored the minimally invasive cohort $[82.3 \%$ versus $90.6 \% \mathrm{P}=0.4506)]$. The mean improvement in pre-operative and post-operative VAS scores for the open cohort and minimally invasive cohort were 6.0 and 5.5, in that order. The mean hospital stay for the open cohort was 64.1 hours, and the mean hospital stay for the minimally invasive cohort was 38.2 hours, which was statistically significant $(\mathrm{P}=0.0001)$.

Conclusion: Our study found that minimally invasive procedures for extraforaminal disc herniation is associated with less complications and shorter hospital stay, but slightly higher re-operation rates. There was no significant difference in pain outcomes or MacNab's criteria. Surgeons should be aware of the differences between these types of operations and use this data to select patients on a case-by-case basis.

316. Pseudarthrosis in Patients Undergoing Multilevel Posterior Cervical or Cervical-Thoracic Fusions: Multi-Center Analysis

Eeric Truumees, MD; Devender Singh, PhD; Matthew Geck, MD; John K. Stokes, $M D$

Introduction: Pseudarthrosis after multilevel posterior cervical or cervical-thoracic fusions is a common complication. We investigated the effect of pseudarthrosis on cervical aligment. Futhermore, we report the effect of type of bone grafts on the rate of pseudarthrosis in patients undergoing multilevel posterior cervical or cervical-thoracic fusions.

Methods: We assembled a multicenter radiographic and clinical database of patients that had undergone 3 or more level posterior cervical or cervical-thoracic fusions with at least 2 years of postoperative (post-op) follow-up. Patients were divided into two groups: group I (fusion ending in the cervical spine) and group II (fusion extending into the thoracic spine). For the analysis, bone grafts were divided into four groups: local only; local and allografts; bone morphogenetic protein (BMP) only; and iliac crest only.

Results: Rate of pseudarthrosis in group I and group II were $21.2 \%$ and $10.96 \%$, respectively. Overall, $53.3 \%$ of the patients with pseudarthrosis were current smokers. The rate of smoking in the solid fusion group was $21.9 \%$. The odds ratio of pseudarthrosis for a smoker compared with a non-smoker was 4.071 (95\% CI: $1.798-$ 9.221). Mean T1 slope for patients with pseudarthrosis increased (2 wk vs. 2 year post-op) in both groups $(\mathrm{p}<0.05)$. Both groups with pseudarthrosis had higher mean $\mathrm{C} 2-\mathrm{C} 7$ sagittal plumbline at 2 years follow-up $(\mathrm{p}<0.05)$. Mean cervical lordosis decreased in both groups with pseudarthrosis ( 2 wk vs. 2 year post-op; $p>0.05$ ). Overall, ANOVA showed no significant effect of type of bone grafts on the rate of pseudarthrosis $(\mathrm{p}>0.05)$.

Conclusion: We conclude that pseudarthrosis affects cervical alignment in patients undergoing multilevel posterior cervical or cervical-thoracic fusions. The study did not find any significant effect of type of bone grafts on the rate of pseudarthrosis. Prospective studies with greater statistical power are needed to further understand the implications of pseudarthrosis on cervical alignment.
317. Transforaminal Lumbar Interbody Fusion (TLIF) With Crescent Shaped, Expandable Lordotic Cage Provides Improved Lumbar Lordosis Compared to Static Cages, and Equivalent Lordosis when Compared to Tradition Smith-Petersen/Ponte Type Osteotomies

Akil Patel, MD; Joshua Alexa, BS, MD, Evan Lewis, MD; Charles A. Sansur, MD, MHSc; David M. Ibrahimi, MD

Introduction: Maintaining and restoring lumbar lordosis has been shown to improve sagittal spinal alignment, thus improving patient outcomes. Several techniques are used to enhance the degree of lordosis, including Smith-Petersen/Ponte type osteotomies (SPO), pedicle subtraction osteotomy (PSO), and vertebral column resection (VCR). This study aims to show if the use of an expandable lordotic cage during transforaminal lumbar interbody fusion (TLIF) produces equivalent or improved lumbar lordosis when compared to the use of static cages and/or stand alone SPOs.

Methods: A retrospective review of 54 patients yielding a total of 84 levels underwent standard TLIF with placement of a crescent shaped, expandable lordotic cage (Globus AlteraTM), at either single or multiple levels An 8 or 15 degree cage was used and expanded to its maximal potential (maximum expansion of $4 \mathrm{~mm}$ ), followed by posterior compression. The degree of lordosis change at each fused vertebral level and overall correction on pre- and post-operative standing scoliosis x-rays were then evaluated. Chart review was conducted to evaluate for post-operative nerve root complications.

Results: On average, a single level expandable interbody cage provided 4.92 degrees of positive lumbar lordosis change per level, and 7.65 degrees of positive lordotic change per patient. On chart review, there were no documented nerve root injuries causing permanent radiculopathy or new weakness.

Conclusion: Expandable, crescent shaped lordotic interbody cages offer greater lordosis correction when compared to static cages and comparable correction to SPOs. Additionally, these cages provided this benefit with no post-operative nerve root injuries. Therefore, expandable cages can be routinely used for comparable lordotic correction providing less overall morbidity. Ultimately, this raises the question of whether the use of these cages coupled with standard SPOs would provide higher achieved lumbar lordosis and obviate the need for more morbid osteotomies including PSO and VCR.

318. A Retrospective Observational Study on the Treatment Outcomes of 26 Patients with Spinal Cord Astrocytoma Including Two Cases of Malignant Transformation

Yong-Eun Cho; Seong Jun Ryu; Dal-Sung Ryu; Kyung-Hyun Kim; Jeong-Yoon Park; Sung-Uk Kuh; Dong-Kyu Chin; Keun-Su Kim; Se Hoon Kim

Introduction: To determine the biologic behavior and prognostic factors of spinal cord astrocytoma, we reviewed surgical and clinical outcomes. Due to the rarity of spinal cord astrocytoma, there is a lack of research regarding this type of tumor and malignant transformation.

Methods: We retrospectively reviewed the data from all patients on whom we performed spinal cord tumor removal between 1983 and 2014. Twenty-six patients were pathologically confirmed to have spinal cord astrocytoma or glioblastoma. Surgical extent and disease progression were confirmed by the surgeon based on operative findings, postoperative MRI, and outpatient department (OPD) follow-up.

Results: Pain or neurological deficit was the chief complaint for all patients. With MRI studies, there is a tendency for high-grade astrocytomas to show as enhanced and heterogeneous images. Two of the low-grade astrocytomas showed malignant transformation over the course of 4 and 11 months, respectively. The overall survival (OS) for low-grade astrocytoma was 28-480 months (mean 156.38 
months); the OS for high-grade astrocytoma was 1-36 months (mean 12.00 months).

Conclusion: Two of 12 low-grade cases showed malignant transformations at 4 and 11 months, respectively, based on pathological confirmation. With spinal cord astrocytomas, enhanced MRI results appeared similar to those of a malignant lesion. We suggest close observation and image correlation of low-grade astrocytomas, even when pathologically confirmed as low-grade. In this review, we found that histologic grade is the most important prognostic factor, although it is not always concordant with biologic behaviors.

\section{Application of Direct Reprogramming Technology to Treat Malignant Spinal Cord Glioma}

Yoon Ha, MD, PhD; Jin Soo Oh; Dong Ah Shin; Seong Yi, MD, PhD; Keung Nyun Kim, MD, PhD; Do-Heum Yoon, MD

Introduction: Glioma is the most malignant type of primary central nervous system tumor and has an extremely poor prognosis. The pathologic characteristic of glioma is poorly differentiated glial cells. Therefore, one potential therapeutic approach is to induce the terminal differentiation of glioma cells through the forced expression of pro-neural factors.

Methods: Rat C6 glioma cells were directly reprogramed in vitro by application with C-AMP inducer forskolin and GSK3 inhibitor CHIR99021. Cell proliferation assay was performed to investigate the role of small molecules on anti-proliferative effect. Immunocytochemistry for MAP2, Nestin, GFAP, NG2 were performed to identify the expression of neural cytoskeletal markers. Whole-cell patch clamp was performed to investigate the expression of neuron specific Na channel. Effect of small molecules on spinal cord tumor in vivo rat model was investigated.

Results: malignant glioma cells can be converted into fully differentiated neurons with no malignant characteristics (i.e., post-mitotic neurons termed small molecule-induced neurons, or SMiNs) through the combined action of two small molecules (GSK3 inhibitor [CHIR99021] and cAMP activator [forskolin]) in the absence of neural transcription factors. Combined, CHIR99021 and forskolin also effectively and consistently inhibited the proliferation of malignant glioma. Gene ontology and gene expression profiles relating to cell division were significantly down-regulated in SMiNs. In vivo, the combined treatment with CHIR99021 and forskolin markedly delayed neurological deficits, and significantly reduced the tumor volume. Combination treatment with small molecules led to various changes involving gene expression, protein expression, signal transduction pathways, and physiological and morphological changes in glioma cells

Conclusion: Direct reprogramming based on small molecules can be applied to suppress the proliferation of malignant glioma. We suggest that the combination of a GSK3 inhibitor and cAMP inducer would be a candidate molecule for the next-generation of anticancer drug for glioma treatment. In addition, reprogramming technology may be a potential treatment strategy, replacing the therapeutic paradigm of traditional treatment of malignant glioma.

320. Diagnostic Utility of Intraoperative Neurophysiologic Monitoring for Intramedullary Spinal Cord Tumors Systematic Review and Meta-analysis

Tej Deepak Azad, BA; Arjun Vivek Pendharkar, MD; Viet Nguyen, MD; James Pan; Ian David Connolly; Anand Veeravagu, MD; Rita Popat, PhD; John K. Ratliff, MD, FACS; Gerald A. Grant, MD

Introduction: Surgical management of intramedullary spinal cord tumors (IMSCT) can involve key neurologic and vascular structures.
Intraoperative neurophysiologic monitoring (IONM) aims to assess the functional integrity of susceptible elements in real time. This study evaluates the diagnostic utility of IONM for IMSCT resection.

Methods: We performed a systematic review of the PubMed and MEDLINE databases for studies investigating the use of IONM for IMSCT and conducted a meta-analysis of diagnostic capability.

Results: Our search produced 257 citations. After application of exclusion criteria, 21 studies remained, ten American Academy of Neurology (AAN) grade III and 11 AAN grade IV. We found that a strong pooled mean sensitivity of $90 \%(95 \% \mathrm{CI}, 84-94)$ and a weaker pooled mean specificity of $82 \%$ (95\% CI, 70-90) for motor evoked potential (MEP) recording changes. Somatosensory evoked potential (SSEP) recording changes yielded pooled sensitivity of $85 \%(95 \% \mathrm{CI}$, $75-91)$ and pooled specificity of $72 \%(95 \% \mathrm{CI}, 57-83)$. The pooled diagnostic odds ratio for MEP was 55.7 (95\% CI, 26.3-119.1), and 14.3 (95\% CI, 5.47-37.3) for SSEP. Bivariate analysis yielded summary ROC curves with AUC of $91.8 \%$ for MEPs and $86.3 \%$ for SSEPs.

Conclusion: MEPs and SSEPs appear to be more sensitive for detection of postoperative injury, though not as specific. Patients with perioperative neurological deficits are 56 times more likely to have had changes in MEPs during the procedure. We observed considerable variability in alarm criteria and interventions in response to IONM changes, indicating the need for prospective studies capable of defining standardized alarm criteria and responses.

321. Somatosensory Evoked Potentials (SSEP) Changes and Development of New Postoperative Neurological Deficits in 53 Patients Undergoing Surgery for Spinal Cord Tumors

Jaspreet Kaur; Gurpreet Surinder Gandhoke, MD; Kourosh Tavanaiepour, DO; Zachary J. Tempel, MD; Donald Crammond; Jeffrey Balzer, PhD; Parthasarthy Thirumala; Adam S. Kanter, MD

Introduction: Little is known about SSEP monitoring for patients undergoing surgery for spinal cord tumors. We present the incidence of SSEP changes and development of new neurological deficits in 53 consecutive patients.

Methods: Observational cohort study on a prospectively collected database from a single institution. We studied SSEP changes (transient or permanent), type of tumor, baseline electrophysiology (normal or abnormal SSEPs), and patient related variables including age, sex, diabetes, hypertension, smoking, coronary artery disease, peripheral vascular disease and body mass index. We identified new neurological deficits in the immediate $(24 \mathrm{hr}$.) postoperative period.

Results: Twenty-eight male and 25 were female. Mean age was 55.4 years (Std. Dev. 18.15, range 14-87), mean weight $84.2 \mathrm{Kg}$ (Std. Dev. 20, range 46-147), mean BMI 28.3 (Std. Dev. 5.91, range 20-49.12), mean duration of surgery 219 mins (Std. Dev. 103.7, range 67-606), mean length of stay 7 days (Std. Dev. 6.8, range 1-40). All neurological deficits were motor weaknesses attributable to a spinal cord (versus root) pathology. Seven new neuro deficits occurred. Four neurological deficits occurred without SSEP changes and three new neurological deficits occurred with accompanying permanent SSEP changes $(p=0.147)$. SSEP changes were found to have a sensitivity of $43 \%$ and specificity of $83 \%$ in predicting a new post-operative neurological deficit (area under the ROC $=0.63$ ). Classifying the SSEP changes as transient or permanent resulted in a sensitivity of $67 \%$ and specificity of $75 \%$ in predicting a new post-operative neurological deficit, (area under the ROC $=0.70$ ). Three out of 21 with normal baseline and 3/28 with abnormal baseline SSEPs developed a new neuro deficit, $\mathrm{p}=0.9$

Conclusion: Tumor location and change in SSEP (transient or persistent), were not found to be statistically significant predictors of postoperative neurological deficit in patients undergoing surgery for spinal cord tumors. 


\section{Independent Osteo-tropic Metastogenic Clones in Polymetastatic Renal Cell Carcinoma}

Nelson Moussazadeh, MD; Samuel H. Berman; Ilya Laufer, MD; Mark H. Bilsky, MD; Jorge Reis-Filho; Cameron Brennan, MD

Introduction: Genomic factors predictive of osteo-tropism have been hypothesized and partially established in several models of cancer. However, the evolutionary dynamics at work in metastatic carcinoma have yet to be characterized in detail. We identified a cohort of clear cell renal cell carcinoma (RCC) patients who also had multiple resections of bone and soft tissue metastases, and performed deep sequencing and computational subclonal analyses to infer phylogeny and essential genetic features acquired prior to systemic dissemination and site-specific colonization.

Methods: Exome capture and deep sequencing was performed on tissue from 3 patients with polymetastatic RCC (including 12 metastases, multiple regions of primary tumors, and paired germline tissue) to a mean depth of 250x. Somatic point mutations were called with Mutect, and insertions and deletions with Strelka and VarScan. Validation sequencing was performed with a custom NimbleGen panel hybridized to a custom sequence library and sequenced to a mean depth of $>500 x$. Allele-specific copy number and clonal prevalence were established using ABSOLUTE, and analyzed with Pyclone across primary and metastatic lesions to determine clonal architecture.

Results: Phylogenetic reconstruction identified ancestral clones, with attendant driver mutations in RCC tumor suppressors (including VHL, SETD2, PBRM1, MTOR etc) and independent subclonal populations in the metastases of all 3 patients. In an index case with multiple soft tissue, spinal and other bone metastases separated spatially and temporally, bone and soft tissue metastases demonstrate apparent independent ancestors. Convergent loss of known tumor suppressors was also noted in all cases, and in several cases these were found in conjunction with de novo mutations in known RCC driver genes acquired late in tumor development.

Conclusion: In this demonstration of subclonal and evolutionary analysis of multiple paired bone and soft tissue RCC metastases, we identified subclonal populations characterized by alteration of several tumor suppressors which subsequently exhibited osteo-tropism.

\section{Micro-Invasive Uninstrumented Spinal Tumor Decompression (MUST-D) for Spinal Metastases}

Dominic Anthony Nistal, MD, FACS; Stanislaw Sobotka, PhD; Kyle J. Riley, BS; Asif Javed; Jordan Patrick Hall

Introduction: We investigated whether minimally invasive vs. open approaches to tumor resection can reduce morbidity, correct deformity, and provide lasting benefit.

Methods: Patients with a diagnosis of cancer and MRI evidence of metastatic epidural spinal cord compression underwent minimally invasive surgery (MIS). Outcomes were compared against controls undergoing open surgery.

Results: 30 patients underwent 32 MIS procedures vs. 51 controls undergoing open surgery. Average surgical time (hours) was 2.8 in the MIS group vs. 4.43 in the open group, average EBL $(\mathrm{ml})$ was 592.83 vs. 988.03 , average hospital stay (days) was 6.69 vs. 10.05, and average time to ambulation (days) were 1.38 vs. 5.51. Among patients with poor ambulation one-month post-op, MIS patients improved significantly by an average of 2.25 Hauser score levels vs. control showing no improvement. Among MIS patients, Cobb angle decreased significantly vs. control showing no improvement. Among open patients there were $7 \mathrm{DVT}, 5 \mathrm{PE}$, and 6 wound infections in the first 30 days vs. none in the MIS group. In the MIS population the average period between recurrences was 6 months; those with surgical recurrence within 4 months of the initial procedure did not undergo any further procedures; they survived for an average of 4.7 months. Those with a recurrence more than 4 months after the index procedure survived more than 28 months on average, underwent an average of 3.75 procedures each, with an average period between re-operations of 6.9 months. Survival correlated to their JSI scores, which was significantly higher in the MUST-D group than the open group, although all of the other tumor scoring or co-morbidity scores were not significantly different pre-operatively to suggest any other differences between the two groups.

Conclusion: MIS had shorter procedures, hospital stays, reduced EBL, better improvement in spinal deformity, increased improvement in ambulation status when compared to open surgery. Low rates of infection and other post-operative complications improve the quality of life for patients in general. Survival differences between the two groups were predicted by their Jenkins Survival Index scores rather than their local disease severity or any other accepted tumor index scores.

\section{Management of Extranodal Lymphoma of the Spine: A Study of 30 Patients}

Shamsudini Hashi, BS; C. Rory Goodwin, MD, PhD; A. Karim Ahmed; Taylor Elise Purvis, BA; Nancy A. Abu-Bonsrah, BS; Benjamin D. Elder, MD, PhD; Genevieve Crane; Daniel M. Sciubba, $M D$

Introduction: Patients with lymphoma involving the spinal column may present with debilitating pain, neurological compromise resulting from spinal cord compression, or loss of spinal integrity. We sought to compare the clinical presentation, treatment, and posttreatment clinical outcomes of patients diagnosed with malignant lymphoma of the spinal column who underwent either medical or surgical intervention.

Methods: The medical records of 30 patients with histologically proven spinal lymphoma were retrospectively reviewed for demographic information, presenting characteristics, treatment, and outcome data. This study excluded patients who failed medical clearance for surgery and patients with insufficient follow-up data.

Results: Eleven patients were managed with chemotherapy and/or radiotherapy, and 19 patients underwent surgical intervention $(84 \%$ of surgical patients with an unknown diagnosis at time of surgery). All surgical patients received postoperative adjuvant therapy with $21 \%$ also being exposed to adjuvant therapy preoperatively. The median length of follow-up was 16 and 3 months for the medical and surgical groups, respectively. In both groups, the thoracolumbar spine was the most common site for these lesions. In patients undergoing surgical intervention, 4 patients were non-ambulatory at baseline with only one of these patients surviving beyond 6 months. All living patients with complete follow-up data $(n=14)$ saw an improvement or a preservation of neurological function at one year, regardless of treatment group. The overall mean survival was 87.6 months with a mean survival of 100.4 and 79.6 months for the medical and surgical groups, respectively.

Conclusion: For this study cohort, indications to undergo surgery included: emergent neurological deterioration, mechanical stabilization of the spine, refractoriness to medical management, or most frequently performing an open biopsy to obtain a pathological specimen. Comparison of medical and surgical management found no significant difference in the initial presentation and clinical outcomes following treatment. However, future prospective studies on patients with surgical indications are needed to provide further information. 
325. Complications Associated with Prolonged Length of Stay After Surgery for Metastatic Spinal Tumors

Alejandro Ruiz-Valls; C. Rory Goodwin, MD, PhD; Rafael De la Garza-Ramos, BA; Taylor Elise Purvis, BA; Nancy A. Abu-Bonsrah, $B S$; Benjamin D. Elder, MD, PhD; A. Karim Ahmed; Ilya Laufer, MD; Chetan Bettegowda, MD, PhD; Daniel M. Sciubba, MD

Introduction: Metastatic disease to the spine constitutes an increasing problem in healthcare due to the morbidity associated with its course and treatment, as well as the sequelae derived from the latter. With concern for the increasing cost of healthcare, the identification of factors associated with prolonged length of stay (PLOS), and its consequences have gained extreme relevance in the management of this subset of patients. The objective of this study is to determine factors that lead to complications associated with prolonged length of stay in patients that underwent surgery for resection of spinal metastasis.

Methods: For this case-control study the United States Nationwide Inpatient Sample (NIS) database was queried from 2002 to 2011. Inclusion criteria were patients who underwent surgery for a metastatic spinal tumor derived from primary lung, breast, kidney, prostate, colorectal, liver, or thyroid cancer. Multiple logistic regression analyses were used to identify predictors of PLOS.

Results: PLOS was defined as an inpatient stay over 14 days postoperatively ( $19.7 \%$ of 5,116 patients). Patients in the PLOS group were slightly younger as compared to the non-PLOS $(\mathrm{p}<0.018)$. Statistical significance was observed with primary tumor locations (i.e., histology), proportion of patients with pathological fracture, presence of visceral metastasis, proportion of patients who underwent internal fixation, and proportion of patients who required transfusion between the PLOS and the non-PLOS groups $(\mathrm{p}<0.001)$. The complication rate was higher in the PLOS group with higher incidence of pleurisy/ pneumothorax/pulmonary collapse, adult respiratory distress syndrome, pneumonia, unplanned intubation, acute renal failure, sepsis, pulmonary embolism, and delirium $(\mathrm{p}<0.001)$. Average total hospital charges for patients with PLOS were $\$ 232,123 \pm 163,851$ compared to $\$ 116,667 \pm 92,701$ for patients who did not experience PLOS $(\mathrm{p}<0.001)$.

Conclusion: Patients with prolonged length of stay may have a higher risk of developing postoperative complications including pneumonia, unplanned intubation, sepsis, pulmonary embolism, and delirium.

\section{Preoperative Risk Stratification in Spine Tumor Surgery -} A Comparison of Three Assessment Scores

Nikita Lakomkin, BA; Blaine Stannard; Scott L. Zuckerman, MD; Justin Virojanapa, DO; Joaquin Camara-Quintana; Luis Kolb, MD; Julio Montejo; Joseph S. Cheng, MD, MS

Introduction: The perioperative care of patients undergoing spinal tumor resection remains challenging. At present, few risk assessment tools have been validated for use in spine tumor surgery. The purpose of this study was to compare and validate the utility of several preoperative assessment scores in predicting outcomes following spinal tumor resection.

Methods: The 2008-2014 National Surgical Quality Improvement (NSQIP) database was used to identify all patients undergoing surgical resection of intramedullary, intradural extramedullary, and extradural spine lesions via CPT codes. American Society of Anesthesiologists (ASA) score, Charlson Comorbidity Index (CCI), and modified frailty index (mFI) were computed for each patient. A binary logistic regression model was used to explore the relationship between these variables and postoperative outcomes including mortality, major complications (Clavien IV), minor complications, and hospital length of stay (LOS). Other significant variables such as demographics, operative time, body mass index (BMI), and tumor location were controlled for in each model. The c-statistic was computed to assess the predictive capacity of the regression models.

Results: A total of 2,170 patients were identified. Of these, 97 (4.5\%) died within 30 days of surgery. Higher CCI scores were independent predictors of mortality $(\mathrm{OR}=1.40, \mathrm{P}<0.001)$, major adverse events $(\mathrm{OR}=1.15, \mathrm{P}=0.016)$, minor complications $(\mathrm{OR}=1.26$, $\mathrm{P}<0.001)$, and prolonged LOS $(\mathrm{OR}=1.20, \mathrm{P}<0.001)$. Patients' $\mathrm{mFI}$ scores were significantly associated with major complications and LOS, but not mortality or minor adverse events. ASA scores were not associated with any outcome metric when controlling for other variables. The computed c-statistic of the models ranged from 0.71 to 0.88 .

Conclusion: The CCI may be valuable as a preoperative tool for predicting an array of outcomes following spine tumor resection and provided superior predictive capacity to $\mathrm{mFI}$ and ASA scores. The validation of assessment scores is important for risk stratification and improving surgical outcomes in these high-risk and rare groups.

327. Predicting Survival in Patients with Spinal Metastatic Disease: A New Survival Index

Dominic Anthony Nistal; Daniel Wei; Stanislaw Sobotka, PhD; Michael Martini; Dominic Anthony Nistal, MD, FACS

Introduction: Spinal metastases have high variability in morbidity and mortality. Existing prognostic scoring systems, including the Tomita and Tokuhashi scores, have limited predictive value for survival of these patients. To improve the prediction of outcomes from treatments, for patients with spinal metastases, we have developed a survival index with superior prognostic value.

Methods: A retrospective analysis on 81 surgically treated patients with similar pre-operative parameters [inclusive of cord compression causing symptoms, excluding those with more than two levels locally symptomatic, and more than two discrete sites causing spinal cord compression] who received surgery for metastatic tumors to the spine. A survival index (Jenkins Survival Index, JSI) was derived from several preoperative assessments, including ambulation and functional indices and tumor burden assessments of six organ systems, to determine predictive value, compared to the scores of Tomita and Tokuhashi.

Results: Pre-operative lung tumor burden (a 0-3 score) and Hauser ambulation index (HAI, 0-9 score) were predictors of survival $(\mathrm{p}=.0025, \mathrm{p}<.0001)$. Using a "machine learning" algorithm, the JSI was found to be optimized by combining the HAI score and four times the lung tumor burden score (JSI, 0-21). The JSI was most significantly associated with survival time $(\mathrm{Rho}=0.588, \mathrm{P}<0.0001)$ with superior positive predictive value compared to either Tokuhashi and Tomita scales as well as several other existing indices of tumor, tissue diagnosis, or co-morbidities.

Conclusion: The JSI predicts survival for patients with a subset significant spinal metastases. This will allow physicians treating this subset of patients to better make treatment decisions and to identify the factors that are most influential in their care. We will validate our system to patients in a larger pool of oncology patients suffering from a more broad degree of metastatic disease, with or without spinal involvement, but in the interim, for patients with a similar degree of spinal involvement, this index can be more predictive than any other existing index. 
328. Application of Morphometrics as a Predictor for Survival in Patients with Prostate Cancer Metastasis to the Spine

Hesham Mostafa Zakaria, MD; Lara Walsh Massie, MD; Azam Basheer, MD; David Boyce-Fappiano; Erinma Elibe; Lonni Schultz, PhD; Farzan Siddiqui; Brent Griffith, MD; Ian Yu Lee, MD; Victor Chang, $M D$

Introduction: Surgery for spinal metastases can improve survival and reduce pain, but has high morbidity that can diminish the surgery benefits and hasten demise. 1 With the limited prognostic ability of Tokuhashi and Tomita scores,2-4 new objective ways of predicting overall survival are necessary. Analytic morphometrics quantifies patient frailty and can predict overall survival in lung cancer patients with spine metastases.5 This study evaluates whether morphometrics is predictive of survival in prostate cancer patients with spinal metastasis.

Methods: Utilizing a retrospective registry of spinal metastases patients who have undergone stereotactic body radiation therapy, we identified females with primary prostate cancer. Morphometric measurements of the psoas muscle were taken from the most recent lumbar spine CT. Patients were stratified into lowest, middle, and highest tertiles based on psoas muscle area. The primary outcome measure was overall survival from the date of CT scan. Hazard ratios were estimated using cox proportional hazards regression analysis.

Results: A total of 92 patients were identified, 52\% African American and 39\% Caucasian. The median survival for all patients was 124 days $(95 \% \mathrm{CI}=98$ 197d). Patients in the smallest third for average psoas size had significantly shorter survival as compared to the largest third: 117 days vs 302 , hazard ratio 2.42 (95\% CI $=1.32$ 4.43), $\mathrm{p}=0.004$. The shorter survival was also true for the middle third as compared to the largest third of psoas size: 113 days vs 302, hazard ratio $2.31(95 \% \mathrm{CI}=1.25-4.25), \mathrm{p}=0.007$.

Conclusion: In prostate cancer patients with metastases to the spine, morphometric analysis of psoas muscle size can be used to identify patients who are at risk for shorter survival. This simple method has the potential to allow clinicians to accurately risk stratify patients based on expected survival. This can aid in surgical decision making by allowing surgeons to weigh a given patient's expected survival and fitness versus the potential morbidity of intervention.

\section{Surgical Timing Affect Survival in Patients with Cervical Metastasis}

\section{Jong-hyeok Park; Sun-Ho Lee; Eun-Sang Kim; Whan Eoh}

Introduction: The purposes of this study are to investigate affecting factors and overall survival between initial Radiation therapy prior to surgical treatment (Group 1) and radiation therapy following initial surgical treatment (Group 2) at the diagnosis of cervical metastasis.

Methods: A retrospective analysis of medical records was performed on 36 cervical metastatic patients from February 2007 to December 2015. Overall survival (OS), OS after cervical metastasis, OS after surgery, neurological and pain outcomes were analyzed between Group 1 and Group 2. Affecting factors of overall survival included; primary tumor type, initial treatment modality, Tomita score, Eastern Cooperative Oncology Group, Karnofsky performance scale (KPS), Nurick grade, Frankel classification, preoperative symptom and Spinal stability neoplastic score.

Results: Both groups showed improvement of postoperative VAS. The difference of pre- and post-operative JOA score was $1.3 \pm 1.9$ in Group $2(p=0.03)$. OS after cervical metastasis was 7.0 months in Group 1 and 15.8 months in Group 2. OS after surgery was 4.5 months and 15.3 months in each Group. There was statistical significance of OS after cervical metastasis in each Group $(p<0.05)$. Factors related to overall survival after cervical metastasis were primary tumor type, initial treatment modality and preoperative symptoms $(\mathrm{p}<0.05)$.

Conclusion: Surgery had a good effect on pain control. The improvement of post-operative JOAS was better in Group 2. Surgery could provide longer OS after cervical metastasis. Early surgery in patients expected good prognosis before neurologic deficit may be a good decision in confined to cervical metastasis.

\section{Racial Disparities in Receiving Surgery and Survival in Patients with Primary Osseous Spinal Neoplasms}

Sayantan Deb, BA; Matthew Schoen; Arjun Vivek Pendharkar, MD; Sean Altekruse, DVM, MPH, PhD; John K. Ratliff, MD, FACS; Atman Desai, $M D$

Introduction: Osseous spinal neoplasms are aggressive tumors that include osteosarcoma, chondrosarcoma, Ewing's Sarcoma, and chordoma. Surgical resection is associated with improved survival for patients with these tumors. However, the effect of race on receiving surgery has not been studied.

Methods: 1779 patients from the SEER program at the National Cancer Institute database were included in this study. All patients were diagnosed with osseous spinal neoplasms of the spinal cord, vertebral column, pelvis, or sacrum from 2003 through 2012. Race was reported as Caucasian or other. Treatment included surgery (gross total resection and other surgery), and no surgery. Logistic regression and Cox proportional hazards models were used to analyze data with SAS v9.4. Results were adjusted for age at diagnosis, sex, socioeconomic status, tumor size, and tumor grade. Kaplan Meier survival curves were constructed according to surgery status and race.

Results: The study found that Caucasian patients were significantly more likely to receive surgery $(\mathrm{OR}=3.977, \mathrm{p}=0.0024)$. Furthermore, non-Caucasian race was associated with significantly shorter survival time $(\mathrm{HR}=1.815, \mathrm{p}=0.0269)$ [Figures 1.0 ]. Receiving surgery was associated with improved overall survival $(\mathrm{HR}=3.001, \mathrm{p}<0.0001)$ [Figure 2.0]. When adjusted for receiving surgery, the effect of race on survival was not significant $(\mathrm{HR}=1.575, \mathrm{p}=0.2079)$.

Conclusion: In this national study of patients with osseous spinal neoplasms, a significant correlation was found between race and the likelihood of receiving surgery. The study also found race to be a significant predictor of overall survival, but this association disappeared when adjusted for receiving surgical treatment. These findings suggest an effect of race on receiving treatment, and consequently, survival, in patients with osseous spinal tumors, regardless of socioeconomic status. Further studies are required to understand reasons underlying this racial disparity, and how they may be addressed.

\section{Distal Hard Palate Resection Allows for Increased Odontoid Resection from an Endoscopic Transnasal Approach Without Significant Dysphagia or Airway Complications}

Joshua E. Loewenstein, MD; Edward Yap, MD; Brian Thorpe; Deb A. Bhowmick, $M D$

Introduction: Feasibility of endoscopic transnasal odontoidectomy is dictated by anatomic variations of the location of the odontoid in relation to the nasopalatine line (NPL) and associated nasopalatine angle (NPA). We hypothesized a limited, dorsal transnasal hard palate resection would increase the NPA to allow for greater resection of the odontoid process for treatment of anterior craniocervical junction pathology.

Methods: Retrospective review of preoperative imaging of 119 patients treated for pathology of the craniocervical junction at our 
center over the past 8 years was performed to estimate the change of the NPA and increase in access to the $\mathrm{C} 2$ vertebral body if a transnasal approach was employed with a $5 \mathrm{~mm}$ distal hard palate resection. This was followed by a clinical review of 8 patients undergoing distal hard palate resection and odontoidectomy over the past 2 years at our institution. Change in NPA, rates of postoperative dysphagia, length of hospital stay, and rate of neurological decline were reviewed.

Results: A mean increase in NPA of $15.4^{\circ}$ was noted with a hypothetical hard palate resection of $5 \mathrm{~mm}$. This increased the potential caudal extent of resection by an average of $20 \%$. Of the 8 patients in which hard palate resection was performed, no significant worsening of swallowing mechanics was noted on postoperative speech evaluation. One patient experienced worsening of neurological function postoperatively followed by early recovery. All patients were extubated immediately postoperatively without need for delayed reintubation. The average hospital stay was 5.4 days.

Conclusion: Resection of the posterior hard palate significantly increases the extent of resection that can be performed by transnasal odontoidectomy, possibly obviating the need for transoral resection in most ventral craniocervical pathology. The adjunctive procedure appears to be well tolerated in the sample of patients in which we treated.

332. A Comparison of Operative Time with Conventional Fluoroscopy Versus Spinal Neuronavigation in Instrumented Spinal Tumor Surgery

James A Miller; Andrew J. Fabiano, MD

Introduction: Spinal metastases are common, occurring in 30\% of cancer patients (1-3). Instrumented spinal stabilization can preserve neurological function and reduce pain in select oncologic patients (4-6). Spinal neuronavigation improves screw placement accuracy $(7,8)$; however its use in oncological operations remains relatively unstudied. Concern exists that utilizing spinal neuronavigation will prolong operative time $(9,10)$.

Methods: Consecutive instrumented oncologic spinal operations were retrospectively reviewed. Patients were placed in two groups based on the method used for pedicle screw placement: 2-dimensional fluoroscopy versus spinal neuronavigation with 3-dimensional imaging. These groups were compared to examine age, number of pedicle screws placed, number of laminectomy levels, operative time, estimated blood loss, post-operative discharge day, and need for reoperation due to pedicle screw misplacement.

Results: There were 39 operations examined; 14 utilized 2D fluoroscopy and 25 utilized spinal neuronavigation. The mean ages of the patients were $64.71 \pm 7.21$ and $63.24 \pm 6.95(\mathrm{p}=0.534)$, mean number of pedicle screws placed was $8.07 \pm 1.98$ and $7.84 \pm 1.34(\mathrm{p}=0.667)$, mean number of laminectomy levels was $2.18 \pm 1.25$ and $1.60 \pm 1.02(\mathrm{p}$ $=0.126)$ in the $2 \mathrm{D}$ fluoroscopy and spinal neuronavigation groups respectively. The mean operative time was $200.79 \pm 34.99$ minutes compared with $193.48 \pm 43.77$ minutes $(\mathrm{p}=0.596)$, estimated blood loss was $790.00 \pm 769.61 \mathrm{ml}$ and $389.80 \pm 551.43 \mathrm{ml}(\mathrm{p}=0.068)$, and the mean number of days the patients were hospitalized following the operation prior to discharge was $7.64 \pm 4.63$ and $6.40 \pm 3.23$ (p $=0.331)$ in the $2 \mathrm{D}$ fluoroscopy and spinal neuronavigation groups respectively.

Conclusion: There was no significant difference in length of operative time when spinal neuronavigation was utilized as compared with standard 2D fluoroscopy for instrumented oncologic spinal surgery. There was a trend towards a significant decrease in estimated blood loss in the spinal neuronavigation cases. Concern over operative time should not be a barrier to using spinal neuronavigation in oncologic cases of spinal instability requiring instrumentation.
333. Myositis Following Spine Radiosurgery for Metastatic Disease: A Case Series

Dennis T. Lockney, BS, MD; Angela Jia; Eric Lis; Natalie Lockney; Chengbao Liu; Benjamin Hopkins, BS; Daniel Higginson, MD; Yoshiya Josh Yamada, MD, FRCP; Ilya Laufer, MD; Mark H. Bilsky, MD; Adam Schmitt

Introduction: Spinal stereotactic radiosurgery (SRS) has emerged as an attractive method to deliver high doses of radiation to oligometastatic spinal tumors of radioresistant histology. As this is a palliative therapy, attention to potential radiation toxicities is paramount when counseling patients. The objective of this study is to report a previously undescribed complication, radiation induced myositis.

Methods: Eleven patients with radiographic evidence of myositis following spinal radiosurgery underwent a retrospective chart review. Clinical and pathologic results were collected, including radiation dose, equivalent dose in 2 Gy fractions (EQD2), biologically effective dose (BED), and volume of muscle treated. Treatment toxicities were classified according to the Common Terminology Criteria for Adverse Events (CTCAE) version 4.03.

Results: Eleven patients were reviewed. Eight patients underwent single fraction 24 Gy treatment and three underwent 9 Gy x 3 fractions. The rate of myositis for patients undergoing single fraction treatment was $1.4 \%$ and was $0.9 \%$ for patients undergoing 3 fraction treatment. The median of the mean dose to muscle with myositis was 17.5 Gy. The median EQD2 was 55.1 Gy and the median BED was $82.7 \mathrm{~Gy}$. Median time to development of clinical symptoms was 1.4 months while the median time to imaging evidence was 4.7 months. Five patients received anti-VEGF therapy. Two patients (18.2\%) had CTCAE grade 3 complications.

Conclusion: Radiation myositis following spinal radiosurgery is a rare but important complication. Single fraction treatment schedules and anti-VEGF therapy may be associated with increased risk of myositis. We present the first series of radiation myositis in patients who underwent spinal radiosurgery for metastatic spinal disease.

334. Stereotactic Radiosurgery Versus Surgical Resection for Spinal Hemangioblastoma: A Systematic Review

\section{Kelly Bridges; Khoi Duc Than, MD}

Introduction: Spinal cord hemangioblastomas are benign vascular tumors arising sporadically in approximately $70-80 \%$ of cases. They can also be manifestations of von Hippel-Lindau (VHL) disease, as these patients will often have multiple spinal hemangioblastomas. Historically, surgical management of symptomatic intramedullary hemangioblastomas has been considered the treatment of choice. However, recently, stereotactic radiosurgery has been utilized as an adjuvant therapeutic modality, and some have suggested it may have utility as the primary treatment option for these tumors. Because of the rarity of spinal hemangioblastomas, management options, clinical outcomes, and prognostic factors have not yet been fully elucidated.

Methods: The National Institutes of Health (PubMed) was queried to identify all studies describing treatment of spinal hemangioblastomas. Focus was narrowed to institutional retrospective reviews, and comparisons were drawn regarding outcomes of both stereotactic radiosurgery and surgical resection.

Results: Stereotactic radiosurgery achieves stable or reduced tumor size with relatively little adverse clinical outcome long-term. Meanwhile, surgical resection results in successful removal of the tumor with approximately $96 \%$ stable or improved long-term clinical effect.

Conclusion: Cross-platform analysis has been challenging when comparing efficacy amongst treatment modalities for this rare tumor. For the institutional retrospective reviews that exist, researchers tend to collect and record data in a multitude of fashions, making 
direct comparisons problematic. As such, the authors propose use of a national registry to input data prospectively about spinal cord hemangioblastomas.

335. CyberKnife Stereotactic Radiosurgery for the Treatment of Symptomatic Vertebral Hemangiomas: A Single Institution Experience

Michael Zhang, MD; Yi-Ren Chen, MD, MPH; Steven D. Chang, $M D$; Anand Veeravagu, MD

Introduction: Symptomatic vertebral hemangiomas (SVH) are a very rare pathology that can present with persistent pain or neurological deficits that warrant surgical intervention. Given their relative rarity and difficulty in assessment, we sought to present a dedicated series of SVH treated by stereotactic radiosurgery (SRS) to provide insight into clinical decision-making.

Methods: A retrospective review of a single institution's experience with CyberKnife radiosurgery for SVH from 2004 to 2011 was conducted to determine the clinical and radiographic outcomes following SRS treatment. We report and analyze the treatment course of five patients with seven lesions, two of which were treated primarily by SRS.

Results: Of the five patients studied, four presented with a chief complaint of refractory pain. Three patients reported dysesthesias, and two reported upper extremity weakness. Following radiosurgery, $4 / 5$ patients exhibited improvement of their primary symptoms, three for pain and one for weakness, achieving clinical response after a mean period of 1 year. In two cases there was $20-40 \%$ lesion reduction in size in the most radiographically responsive dimension. All treatments were well tolerated.

Conclusion: SRS for SVH is a safe and feasible treatment strategy, comparable to prior RT studies, and in select cases may successfully confer delayed, decompressive effects. Additional reporting will determine future patient selection and how conformal SRS treatment can best be administered.

336. Low-Dose Stereotactic Body Radiotherapy for Spinal Metastases Has Reduced Vertebral Body Fracture Rates While Maintaining Local and Pain Control

Kevin D. Kelley, MD, PhD; Shashank Gandhi; Emile Gogineni, DO; Cristina Sison, PhD; Katherine Wagner, MD; Beatrice Bloom, MD; Louis Potters, MD; Maged Ghaly, MD; Ahmad Latefi, DO

Introduction: A staple of current treatment paradigm of spinal metastasis is radiosurgery as the primary modality, reserving surgery for high-grade epidural spinal cord compression or spinal instability. Multiple studies report vertebral body compression fracture rates from $11-39 \%$ after SBRT. Reports of patients' pain control ranged from $52-85 \%$ at 12 months and local control rates of $87-95 \%$ at 6 months to $73-92 \%$ at 12 months. Studies have shown that with high treatment doses the fracture rates are higher. We describe our experience of low-dose SBRT for spinal metastasis with fracture rates, local and pain control.

Methods: This is a retrospective, single institution analysis of 134 patients (316 spinal segments) undergoing SBRT for spinal metastases from 2010-2015. Fracture rates were assessed on follow-up CT. Local control analysis conducted with Kaplan-Meier statistics.

Results: 134 patients (316 spinal segments) were included in the analysis. Median age 67 years (17-90). Location of tumors were $13 \%$ cervical, $49 \%$ thoracic, 34\% lumbar, $4 \%$ sacral. Tumor histology was $56 \%$ radiosensitive and $44 \%$ radioresistant. Treatment doses were: $13-20 \mathrm{~Gy}$ single-fraction (54\%); 16-27Gy 3-fraction (36\%); and 25-40Gy 5-fraction (10\%). 54 (17\%) spinal levels required separation surgery for high-grade epidural spinal cord compression. Patient reported pain scores showed $61.8 \%$ improved, $22.4 \%$ stable, and $15.8 \%$ worsened. Fracture rates were $7.3 \%$. Local control rates were $83 \%(77-87 \%)$ at 6 -months and $76 \%(68-81 \%)$ at 12 -months. Local failure rates better in single-fraction versus multi-fraction $(\mathrm{HR}=2.43$ [CI, 1.14-5.16]; $\mathrm{p}<0.0352)$. There was no difference between radiosensitive and radioresistant tumors in local control $(\mathrm{HR}=1.59$ [CI, 0.74-3.46]; $\mathrm{p}<0.264$ ).

Conclusion: Low-dose SBRT can achieve durable radiologic local and pain control in spinal metastatic disease. Fracture rates can be lowered with this approach, while still balancing appropriate local and pain control. Single-fraction treatment is superior to multi-fraction regimens. Multidisciplinary treatment teams can entertain low-dose regimens for patients at higher risk for vertebral body fractures.

\section{Does Kyphoplasty Prior to Spine SBRT for Osseous Tumors Affect Local Control? A Retrospective Series Review}

Ori Barzilai, MD; Natalie DiStefano, BA; Eric Lis; Yoshiya Josh Yamada, MD, FRCP; Mark H. Bilsky, MD; Ilya Laufer, MD

Introduction: The goals of treatment for spinal metastases include treatment of the local tumor, restoration of spinal stability and palliation of symptoms. Mechanical instability in spine cancer patients causes pain and discomfort. Treatment of mechanical instability prior to SBRT may be beneficial in facilitating radiotherapy and providing timely pain relief. Kyphoplasty has been proven to be an effective method of pain reduction in both osteoporotic compression fractures as well as in tumor-related compression fractures. Kyphoplasty prior to radiosurgery has been previously described as an effective, non-invasive treatment strategy yet it's affect on SBRT local control remains unknown.

Methods: A retrospective review of charts, radiology reports and images was performed of all patients who received single-fraction spine body radio therapy (SBRT) either as stand-alone or postkyphoplasty at a large tertiary cancer center from January 2012 to July 2015. Patient and tumor variables were documented along with treatment planning data and dosimetry.

Results: Overall, 192 treatments in 164 patients were identified of which17 underwent kyphoplasty prior to SBRT at the treatment level. The median time from kyphoplasty to SBRT was 22 days. Four of 192 treatments $(2 \%)$ demonstrated local tumor recurrence/progression at the time of analysis. Of the 4 , one patient had kyphoplasty prior to radiation. This recurrence occurred 18 months following SBRT in the setting of widespread system ic disease and spine progression. Dosimetric review demonstrated a lower then average treatment dose for this case when compared to the cohort. There were no significant differences in dosimetry analysis between the group of patients who underwent kyphoplasty prior to SBRT and the remaining cohort.

Conclusion: Stabilization with cement augmentation prior to SBRT is safe and does not alter the radiation efficacy nor should it preclude physicians from adhering to SBRT planning and contouring guidelines.

338. Stereotactic Radiosurgery for Intradural Spine Tumors Using Cone Beam Computed Tomography (CBCT) Image Guidance

Alp Ozpinar MD; Andres Monserrate; Benjamin M. Zussman, MD; Ajay Niranjan, MD, MBA; John Flickinger, MD; Peter C. Gerszten, $M D, M P H$, FACS

Introduction: Cone beam computed tomography (CBCT) image guidance technology has been adopted for spine radiosurgery.This 
study prospectively evaluated a series of intradural spine tumors treated with radiosurgery using CBCT.The feasibility of using CBCT guidance for radiosurgery for intradural spine tumors was evaluated.

Methods: Eighty-two patients with intradural tumors were evaluated. The positioning deviations of the spine radiosurgery treatments in patients were recorded. Radiosurgery was delivered using a linear accelerator with a beam modulator and CBCT combined with a robotic couch that allows positioning correction in three translational and three rotational directions. To measure patient movement, three quality assurance CBCTs were performed and recorded in 30 patients: before, halfway, and after the radiosurgery treatment. The positioning data and fused images of planning CT and CBCT from the treatments were analyzed to determine intra-fraction patient movements.From each of three $\mathrm{CBCTs}$, three translational and three rotational coordinates were obtained.

Results: Lesion locations include cervical (22), thoracic (17), lumbar (38) and sacral (5). Tumor histologies included schwannoma (27), neurofibroma (18) meningioma (16), hemangioblastoma (8) and ependymoma (5).The mean prescription dose was 17 Gy delivered in one to three fractions. At the treatment halfway point, the translational variations and standard deviations were $0.4 \pm 0.5,0.5 \pm 0.8$, and $0.4 \pm 0.5$ $\mathrm{mm}$ in the lateral $(\mathrm{X})$, longitudinal $(\mathrm{Y})$ and $\mathrm{AP}(\mathrm{Z})$ directions, respectively. Similarly, the variations immediately after treatment were $0.5 \pm 0.4,0.5 \pm 0.6$, and $0.6 \pm 0.5 \mathrm{~mm}$ along $\mathrm{X}, \mathrm{Y}$ and $\mathrm{Z}$ directions, respectively.The mean rotational angles were $0.3 \pm 0.4,0.3 \pm 0.4$, and $0.3 \pm 0.4$ degrees along yaw, roll, and pitch, respectively, at the halfway point and $0.5 \pm 0.5,0.4 \pm 0.5$, and $0.2 \pm 0.3$ degrees after treatment.

Conclusion: Radiosurgery offers an alternative treatment option for intradural spine tumors in patients who may not be optimal candidates for open surgery. CBCT image guidance for patient setup for spine radiosurgery is accurate and successful in patients with intradural tumors.

\section{Predictors of Complications and Readmission Following} Spinal Stereotactic Radiosurgery

Daniel Lubelski, MD; Joseph E Tanenbaum, BA; Taylor Elise Purvis, BA; Thomas Bomberger; C. Rory Goodwin, MD, PhD; Ilya Laufer, MD; Daniel M. Sciubba, MD

Introduction: Stereotactic radiosurgery (SRS) is increasingly used for treatment of spinal tumor. It is currently unclear what baseline demographic factors predict post-treatment outcomes. The objective of the present study was to identify preoperative factors associated with major morbidity, mortality, increase hospital length of stay (LOS), 30 day readmission and operation rates following SRS for spinal tumors.

Methods: The American College of Surgeons National Quality Improvement Program (NSQIP) was queried from 2012 to 2014 to identify all adult patients that underwent SRS for spinal tumors. Univariate analysis was used to identify potential predictive variables, multiple imputation was performed to account for missing data. Multivariable logistic regression was used to identify independent statistically significant predictors of post-treatment outcomes.

Results: 2714 patients were identified that met inclusion criteria. 184 patinets $(6.8 \%)$ had "major morbidity or mortality," $193(7.1 \%)$ had a major morbidity, $186(6.9 \%)$ were readmitted within 30 days, and $116(4.3 \%)$ had a subsequent operation within 30 days. Age, BMI, and ASA class were predictive of LOS. Major morbidity was predicted by age greater than 80 , BMI $>35$, high ASA class, as well as pre-treatment functional dependence and other baseline comorbidities. Predictors of operation within 30 days included preoperative steroid use, renal failure, $\mathrm{BMI}>35$, and if the treatment was non-elective.

Conclusion: The data herein demonstrate that $4-7 \%$ of patients undergoing SRS for spinal tumors have morbidity following the procedure. A large percentage of this is likely attributable to baseline patient characteristics and severity of their oncologic disease. Factors that are independently predictive of morbidity, increased length of stay, and subsequent operation included age, BMI, and baseline comorbidities and functional status. Identification of preoperative patient-specific factors that are predictive of post-treatment outcome will aid in patient selection and patient counseling leading to greater patient satisfaction and hospital efficiency.

340. Adjacent Level Degeneration in the Lumbar Spine: Biomechanical Evaluation of a Novel Combination Cortical and Pedicle Screw Construct

\section{James Leonard West, BSE; Wesley Hsu, MD}

Introduction: Adjacent level degeneration is a ubiquitous problem after lumbar spinal stabilization which can lead to significant patient morbidity, particularly for patients who require extension of the preexisting fusion to stabilize the adjacent level. A novel hybrid "add on" construct utilizing cortical trajectory screws placed in the same pedicle as preexisting pedicle screws can minimize exposure of preexisting hardware.

Methods: Eight cadaveric lumbar spine specimens $(n=8)$ were obtained from fresh frozen cadavers. The specimens were randomly divided between a traditional pedicle screw fusion group and a hybrid pedicle/cortical trajectory fusion group. Specimens were then tested for range of motion in a robotic system under force/torque control to a moment of $8 \mathrm{Nm}$. Three surgical states (native state, two level fusion, and three level fusion) were evaluated. An optical tracking system was used to record relative displacement and rotation for each vertebra.

Results: The eight cadaveric specimens were instrumented as described. Overall results should reliable decreases in range of motion with instrumentation. There was no statistical significance between range of motion reduction between the hybrid and traditional pedicle screw constructs at the doubly instrumented level. Additionally, when taken as a whole, there was also no significant difference in reduction of range of motion between hybrid and traditional constructs.

Conclusion: Overall, these data support the potential viability of a hybrid cortical / pedicle screw construct on a bio-mechanical basis. This demonstrates a potential avenue to add onto existing lumbar fusion constructs in a minimally invasive fashion with minimal disruption to the pre-existing construct.

341. Patient-Reported Outcomes After Two-Level Anterior Cervical Discectomy and Fusion Versus Single-Level Corpectomy for Cervical Myelopathy: An Analysis of a Quality Outcomes Database Cohort

Taylor Sommer; Jian Guan, MD; Vijay Ravindra, MD, MSPH; Andrew T. Dailey, MD; Robert S. Hood, MD; Meic H. Schmidt, MD; Erica Fay Bisson, MD, MPH

Introduction: Anterior cervical disc and fusion (ACDF) and anterior cervical corpectomy and fusion (ACCF) are among the most common and effective surgical treatments for cervical spondylotic myelopathy. However, none of the previous studies have used a proven prospective instrument with patient reported outcomes and metrics to further elucidate which procedure is best. In this study, we combine patient demographics and patient reported outcomes from the Quality Outcomes Database to produce additional depth into the ACDF vs ACCF discussion.

Methods: Eighty-three patients underwent ACDF or ACCF during 2012-2015. Data was obtained from the Quality Outcomes Database (QOD). Patient reported outcomes (PRO) scores utilized VAS, NDI, TTO, and EQVAS. 
Results: Patients who underwent ACCF were older (62.82 vs $55.92 \mathrm{p}=0.016)$. They were also more likely to have hypertension $(\mathrm{p}=0.047)$ and osteoporosis $(\mathrm{p}=0.012)$. In preoperative PRO scores the ACCF group was less likely to have worse VAS neck scores $(\mathrm{p}=0.012)$, VAS arm scores $(\mathrm{p}=0.002)$, NDI scores $(15.53$ vs $22.23, p=0.017)$, and TTO scores $(p=0.015)$. The ACCF group had longer lengths of hospital stays (3.71 days vs 1.82 days, $\mathrm{p}=0.006$ ), greater estimated blood loss (154.71 $\mathrm{ml}$ vs $57.47 \mathrm{ml}, \mathrm{p}=<0.001)$, and longer operative time (241.41 minutes vs 199.83 minutes, $\mathrm{p}=0.037$ ). Complication rates postoperatively were similar $(\mathrm{p}=0.810)$ as were fusion rates at 12 months $(\mathrm{p}=0.675)$. The postoperative PRO scores at 12 months showed no statistical difference between the two groups both absolutely and relatively.

Conclusion: Patients who underwent ACCF tended to be older, were more likely to have hypertension and osteoporosis, and had poorer preoperative scores. The ACCF procedure led to longer operating times, more blood loss, and longer hospital stays. Despite these differences, PRO scores at 12 months were equivocal between the two groups both in absolute improvement and in relative improvement.

342. Aesthetic Comparison Between Subcuticular Suture and Staple Closure of Anterior Cervical Spine (ACS) Incision Scars: A Prospective Controlled Single-blinded Clinical Trial

Sina Rajamand; Diana Kakos, BSc; Doris Tong, MD; Teck Mun Soo

Introduction: ACS procedures are common and neck scar appearance is important aesthetically. This study compares subcuticular suture with staple closure regarding aesthetics of the neck scar.

Methods: This is a single-blinded comparative prospective controlled study with two cohorts in two hospitals. All consecutive patients who underwent one/two level ACS operation from 9/2015$8 / 2016$ were screened. We excluded patients with postoperative wound infection, reoperation in the same admission, previous ACS operations, non-compliance in follow-up, and inability to give informed consent. We did single layer skin stapling without platysma closure or subcuticular suture with platysma closure. Patients were followed up at 1.5, 3, and 6 months. We used Stony Brook Scar Evaluation Scale (SBSES, 0-5) with 5 being the best score. Digital images were taken in a standardized manner and saved in a secure database. A blinded plastic surgeon and a blinded trained non-healthcare professional evaluated the scars using SBSES. A-priori sample size using a clinically significant difference of 1 was determined. Rank-sum test was used.

Results: In this interim analysis, we studied 94 staple and 47 suture closures. There is no significant difference between the groups regarding age, sex, rate of diabetes, smoking, obesity (BMI $>30$ ), chemotherapy, duration of surgery, and length of incision with significant difference regarding number of cervical levels. The mean interval of staple removal is 13.3 days. There is no significant difference regarding SBSES as evaluated by the plastic surgeon (staples vs. sutures, median 2 vs. 2 , range $0-5, \mathrm{p}=0.63$ ), or nonhealthcare professional (staples vs. sutures, median 4 vs. 2 , range $0-5$, $\mathrm{p}=0.4$ ).

Conclusion: We demonstrated that staple and suture closure achieve equivalent aesthetic outcomes in ACS procedures when evaluated either by a plastic surgeon or a non-healthcare professional in interim analysis.
343. Systematic Review of Patient-specific Surgical Simulation: Next Step in Advancing Medical Education

Won Hyung (Andrew) Ryu, BSc, MSc, MTM, MD; Navjit Dharampal; Ahmed Mostafa; Ehud Sharlin; Gail Kopp; Bradley Jacobs, MD, FRCSC; R. John Hurlbert, MD, PhD, FACS, FRCS(C); Sonny Chan; Garnette R. Sutherland, MD, FRCSC

Introduction: Simulation-based education has been proven to be an effective tool to teach foundational technical skills in various surgical specialties. However, the majority of current simulations are limited to generic scenarios and does not allow continuation of the learning curve beyond basic technical skills to prepare for more advanced expertise such as patient-specific surgical planning. The objective of this study was to evaluate the current medical literature with respect to the current utilization and educational value of patientspecific simulations for surgical training.

Methods: We performed a systematic review of literature using Pubmed, Embase, and Scopus focusing on themes of simulation, patient-specific, surgical procedure, and education. We included randomized controlled trials, cohort studies, and case-controlled studies published between 2005 2016. Two independent reviewers (W.H.R., N.D) conducted the study appraisal, data abstraction, and quality assessments of the studies.

Results: Our literature search identified 13 studies that met our inclusion criteria with 7 studies utilizing computer simulations and 6 studies using 3-dimentional (3D) synthetic models. A wide range of surgical specialties have studied patient-specific simulation including neurosurgery, vascular surgery, orthopedic surgery and interventional radiology. The most common procedures being studied was spinal surgery procedures such as pedicle screw insertion followed by endovascular procedures. However, majority of the studies were small in size and primarily aimed at feasibility assessment and early validation.

Conclusion: Early evidence has shown feasibility, validity and utility in patient-specific simulation for surgical education. With further development of this technology, simulation-based education may be able to effectively improve necessary cognitive skills of residents to aid in their surgical performance.

\section{The Influence of Manual Bending Spinal Rods on Construct Fatigue Life}

Joseph E Tanenbaum, BA; Gabriel Alexander Smith, MD; Stephen Kral, BS; James Barber, BS; Michael P. Steinmetz, MD

Introduction: Surgeons typically manually bend rods when correcting spinal deformity. This bending is thought to possibly work harden the material and thus increase its yield point. However, alternatively, bending may reduce the yield point and thus affect construct fatigue life. Clinically this may result in construct failure.

Methods: Titanium and Cobalt Chrome $5.5 \mathrm{~mm}$ rods were tested in virgin (straight) and bent (10 degrees via French Bender) conditions in a cantilever setup (ASTM F2193-14) to determine their static yield points. Four groups were tested with five samples in each group: (i) Titanium virgin, (ii) Titanium bent, (iii) $\mathrm{CoCr}$ virgin, and (iv) $\mathrm{CoCr}$ bent. Ten degrees was chosen as a worst case condition for this test. Each rod was mounted in the cantilever setup so that the applied load was $50 \mathrm{~mm}$ away from the fixture face at a rate of $25 \mathrm{~mm} / \mathrm{minute}$.

Results: The yield points for both the Titanium and $\mathrm{CoCr}$ bent rods decreased by $42 \%$ when comparing virgin rods to those bent by 10 degrees. The fatigue life curve derived from ASTM F1717 testing for a $5.5 \mathrm{~mm}$ Titanium virgin rod reveals that a construct will survive 5 million loading cycles when loaded to $40 \%$ of yield (2,500 ustrain). It also demonstrates that if the load is increased to $62 \%$ of yield $(4,000$ ustrain) the virgin rod will survive less than 1 million cycles.

Conclusion: The results of the present study suggest that constructs 
made with manually bent rods have significantly lower fatigue life and potentially a greater chance of construct failure relative to virgin rods. A lower yield point will translate to a lower expected fatigue life. Extrapolating to the bent rods, this result implies that using manually bent rods may result in a significantly reduced fatigue life.

\section{Safety and Accuracy of Freehand Versus Navigated C2 Pedicle/Pars Screw Placement}

Randall Hlubek, MD; Michael Bohl, MD; Clinton David Morgan, MD; Tyler Scott Cole, MD; Jay D. Turner, MD, PhD; Udaya K. Kakarla, $M D$

Introduction: $\mathrm{C} 2$ pedicle and pars screws require accurate placement to avoid injury to the nearby neurovascular structures. Freehand, fluoroscopically guided, and CT-based navigation techniques have been described in the literature. The purpose of this study is to compare the safety and accuracy of freehand versus navigated technique for $\mathrm{c} 2$ pedicle/pars screw placement.

Methods: Retrospective review of consecutive patients treated with posterior fixation constructs containing $\mathrm{C} 2$ pars or pedicle screws placed by spine surgeons from 2010 to 2016 at Barrow Neurological Institute. Basic demographic data, intraoperative and postoperative complications, screw diameter and length, method of screw placement (freehand vs. navigated), and incidence of stroke and all-cause mortality within 30 days of the operation were recorded. Accuracy of screw placement was assessed in those patients with intraoperative/postoperative CT imaging available. Screw accuracy was graded independently by two reviewers according to the following criteria: Grade A (no breach of the cortical surfaces), grade B-E (breach with transverse foramen obstruction of 1-25\%, 26-50\%, $51-75 \%, 76-100 \%$ respectively), Grade M (medial breach). Screws were then divided into acceptable placement (Grades A,B) and unacceptable placement (Grades C-E, M).

Results: A total of $426 \mathrm{c} 2$ pedicle or pars screws (312 freehand, 114 navigated) were placed in 220 patients. Three vertebral artery injuries ( 2 freehand, 1 navigated; $p=1), 5$ deaths ( 4 freehand, 1 navigated; $\mathrm{p}=1)$, and 1 stroke in the navigated group ( $\mathrm{p}=.6$ ) occurred. CT imaging was available for accuracy grading of 182 screws (131 freehand, 51 navigated). No breaches (Grade A) occurred in $86 \%$ of the freehand screws and $67 \%$ of the navigated screws $(p=.023)$. More screws had acceptable placement in the freehand group (94\%) than the navigated group $(82 \%)(\mathrm{p}=.023)$.

Conclusion: Freehand is significantly more accurate than CTbased navigation for $\mathrm{c} 2$ pedicle/pars screw placement. There was no difference in complication rate between the two techniques.

\section{The Safety and Efficacy of Outpatient Anterior Cervical Discectomy and Fusion}

Michael Weiss, MD; Zoltan Bereczki, DO; Stefan Prada, MD; Jed Paul Weber; Michael Perry, MD; Chip Wade, PhD; Reginald J. Davis, MD, FACS

Introduction: Anterior cervical discectomy and fusion (ACDF) remains the standard of care for patients with cervical radiculopathy who are unresponsive to conservative medical care. ACDF has been shown to be a safe and effective treatment for a number of spinal disorders [1 2]. Performing an ACDF in an outpatient setting provides an opportunity to reduce the total cost of surgery. Research is widespread on the substantial benefits to outpatient surgeries besides overall reduced costs. However there are few studies that examine the safety and efficacy of performing an outpatient ACDF. The purpose of our study was to evaluate the safety and efficacy of performing an ACDF with instrumentation on an outpatient basis.
Methods: Over a 1-year period from December 2013 to January 2015,80 patients were retrospectively evaluated for the efficacy and safety of performing ACDF with instrumentation procedures on an outpatient basis. The safety and efficacy of performing ACDF as an outpatient procedure were assessed comparing intraoperative and perioperative complications, which were reported for a 1-year followup time period. Clinical parameters, such as surgical blood loss and duration of the surgical procedure were reported. Patient reported outcomes where measured via a Visual Analog Scale (VAS) and Neck Disability Index (NDI).

Results: Overall, $100 \%$ of patients were discharged less than 6 hours after their surgeries. There were 0 reported intra-operative and post-operative complications in the patient population. The average length of surgery was 136 minutes with an average effective blood loss of $59.84 \mathrm{ml}$. Results show a $62 \%$ improvement in the VAS and a $68 \%$ improvement in the NDI.

Conclusion: The findings of the current study indicate that an ACDF performed in an outpatient setting may prove to be beneficial in decreasing the complications and morbidity of standard treatments for with cervical radiculopathy.

\section{Spine Surgery A Mari Usque Ad Mare: Pre-operative Patient Metrics Across Canada}

Godefroy Hardy St-Pierre, MD, FRCSC; Michael M.H. Yang, MD, M.Biotech; R. John Hurlbert, MD, PhD, FACS, FRCS(C)

Introduction: CSORN is the spine registry of the Canadian Spine Society. For the first time, we use this powerful tool to directly compare pre-operative characteristics in patients undergoing thoracolumbar procedures between 5 surgical centers across the country: Vancouver, BC, Calgary AB, Edmonton, AB, Toronto, $\mathrm{ON}$ and St-Johns, NB. Patients were not stratified according to procedure performed.

Methods: We extracted data from the main CSORN database concerning pre-operative metrics. Those 32 variables included patient characteristics (Age, BMI, Smoking, etc.), comorbidities (medical, psych, type of medications, prior spine OR, etc.) and functional indicators (VAS LE, VAS Back, SF12, EQ5D, PHQ9 and ODI). ANOVA was used for preliminary analysis followed by multinomial logistic regression.

Results: There was a total of 1534 patients across the 5 centers. Average age ranged from 37 y.o. in Edmonton to 50 y.o. in Vancouver. Average BMI ranged from 26.4 in Edmonton to 29.9 in St-Johns. Average VAS LE ranged from 6.93 in Toronto to 7.71 in Calgary. Average VAS Back ranged from 6.12 in Toronto to 7.08 in St-Johns. Average ODI ranged from 43 in Toronto to 51 in Edmonton. Univariate analysis revealed statistically significant higher age in Vancouver $(p<0.001)$, higher BMI in St-Johns $(p=0.004)$, lower VAS LE and VAS Back in Toronto ( $\mathrm{p}=0.04$ for both) as well as lower ODI $(\mathrm{p}=0.03)$. There was no significant differences between other variables. The multivariate analysis found no statistically significant difference across centers.

Conclusion: Remarkable homogeneity in pre-operative patient characteristics exists between surgical centers across Canada despite varying surgical caseloads, procedures of choice and focus or lack thereof on academic pursuits. Canadian universal healthcare system might partially explain this intriguing finding. The consistency of patient populations across centers bodes well for the strength and reproducibility of findings from the CSORN registry. 


\section{Prognostic Utility of Pain Diagrams for Predicting Surgical Intervention Among Spine Referrals}

Michael M.H. Yang, MD, M.Biotech; Khaled Almansoori; Godefroy Hardy St-Pierre, MD, FRCSC; Stephan DuPlessis, MD

Introduction: Pain diagrams (PD) have been used as screening tools for the evaluation of back pain but show variability in their diagnostic and prognostic utility. Several studies have compared different PD methods, but none have investigated their role in predicting surgical intervention. Therefore, a prospective cohort study was undertaken to evaluate 5 different PD interpretation methods; Uden, Ransford, Margolis, Ohnmeiss and the Quebec Task Force (QTF) approaches. Primary objectives were to compare the method's likelihood of predicting operative versus non-operative management among patients referred for lower back pain pathologies.

Methods: Consecutive patients meeting inclusion criteria were asked to complete a PD prior to their first encounter with their spine surgeon. Clinical management details were retrospectively collected over a 24 month subsequent period and patients meeting inclusion criteria were evaluated. Statistical analysis of independent and dependent variables were performed using descriptive statistics, Spearman correlative analysis, and binomial logistic regression analysis using SPSS v.22 (SPSS Inc., Chicago, Illinois).

Results: A total of 317 were included in the study of which $12.9 \%$ underwent surgical intervention. Binomial logistic regression showed weak relationships between PD interpretations and surgical intervention as demonstrated by Nagelkerke-variability estimations; Uden $(\mathrm{R} 2=0.32)$, Ransford $(\mathrm{R} 2=0.21)$, Margolis $(\mathrm{R} 2=0.17)$, Ohnmeiss (R2 $=0.15)$ and the Quebec Task Force methods (R2 $=0.31$ ). Positive predictive value was highest among the QTF method $(39.6 \%)$ and negative predictive value was highest with the Uden method $(82.9 \%)$. In general, interrater agreement was highest with the Ohnmeiss method (88.5\%) and lowest with the Margolis method $(66.7 \%)$.

Conclusion: Pain diagrams demonstrate good inter-rater agreement and generally provide negative predictive value for evaluating the likelihood that a patient will undergo operative intervention. However, they are not useful in predicting who will undergo surgery. Their utility In clinical decision making is limited compared to modern day clinical-radiological correlation.

349. Potential Mechanism of Injury During Cervical Spine Procedures as an Etiology for Post-operative C5 Palsy -- An Anatomical Study

Fernando Enrique Alonso, MD; Vlad Voin; David Hanscom, MD; Jens Chapman, MD; Rod J. Oskouian, MD; R. Shane Tubbs, PhD, $P A-C$

Introduction: C5 palsy is a common cause of post-operative deltoid weakness. Multiple studies have described varied techniques in order to decrease its incidence. Inferior shoulder retraction is usually employed in order to assist with surgical visualization during anterior and posterior surgical procedures. Research into the amount of cervical traction generated during shoulder depression is lacking. We hypothesized that depressing the shoulder during cervical spine surgery might result in C5 nerve traction with resultant C5 nerve palsy.

Methods: Ten adult human cadavers underwent dissection of the spinal cord, cervical dorsal/ventral rootlets, spinal nerve, and cervical and $\mathrm{T} 1$ dorsal and ventral rami in the supine and prone positions. Various movements (performed five times each) were made (shoulder depression/elevation, neck flexion/extension, neck lateral flexion, and head rotation). The effects of movements upon the cervical nerve rootlets, nerves, and rami were measured. Video recordings were generated during each movement.
Results: The greatest amount of displacement of nervous tissue was generated by shoulder retraction and was seen primarily at the rootlet level. The nerve rootlets that underwent the greatest average displacement were found at C5 with a decreasing gradient to $\mathrm{C} 7$ and then no gross motion at $\mathrm{C} 8$ or T1. With maximal shoulder depression, $\mathrm{C} 5-\mathrm{C} 7$ rootlet tension produced cord movement to the ipsilateral side again with the $\mathrm{C} 5$ level moving the greatest distance.

Conclusion: Shoulder retraction is often used during cervical spine surgery. In cadavers, shoulder depression places a significant amount of tension and causes displacement of the $\mathrm{C} 5$ nerve rootlets and at an extreme, cord displacement to the ipsilateral side. This may be a potential mechanism for injury placing patients at a greater risk for C5 palsy.

350. Occipital-Cervical Fusion in Patients With Chiari Malformations and Connective Tissue Diseases: A Review of Practices and Guidelines

Mauricio J. Avila, MD; Jeffrey P. Greenfield, MD, PhD; Ali A. Baaj, $M D$

Introduction: Cranio-vertebral junction (CVJ) abnormalities are common in patients with co-morbid Chiari malformation and connective tissue disorders (Ehlers-Danlos, Marfan syndrome etc.). Some of these patients may require occipital-cervical fusion (OCF), however, the indications and the timing of an OCF in this population remains controversial. The aim of this study was to review the literature to determine current practices and guidelines for OCF in patients with Chiari malformations in the presence of a recognized connective tissue disease

Methods: We performed a literature review on the U.S National Library of Medicine via Pubmed. We used Medical Subject Headings (MeSH) when available and the search included: "ArnoldChiari malformation", "skin and connective tissue diseases", "congenital, hereditary, and neonatal diseases and abnormalities", "occipitocervical" and "fusion". We extracted the data looking for: type of study, number of patients, diseases etiology, previous surgeries, and indications utilized for OCF, follow-up and reported complications

Results: The search yielded 226 results, with 215 in English. We excluded 180 articles that didn't have information on OCF, patients with different diagnosis, no separate analysis between different pathologies of the CVJ. 34 articles were chosen for our final analysis. This cohort represented 1548 patients of whom 925 underwent OCF $(59.8 \%)$. The majority of the studies were retrospective case series. Ten common criteria were represented in the literature when discussing $\mathrm{OCF}$ in patients with Chiari and connective tissue diseases. Among these include: neurological dysfunction and a clivo-axial angle $<125-129^{\circ}$, ventral brainstem compression (basilar invagination or a pb-C2 line $>9 \mathrm{~mm}$ ), Chiari I and malformations of the CVJ, Chiari and co-morbid or Morquio syndrome, osteogenesis imperfecta or Klippel-Feil and CVJ instability, a history of prior Odontoidectomy, persistent neurological symptoms after posterior fossa decompression, a diagnosis of Morquio syndrome and no symptoms but progressive instability, and patients with multiple posterior fossa decompressions

Conclusion: This is the most comprehensive review of literature, to date, highlighting existing guidelines and practices for OCF in patients with Chiari malformations and connective tissue diseases. Larger prospective studies are needed to better assess the need and timing for OCF in this challenging and heterogeneous patient population. 
351. Inadvertent Durotomies During Spine Surgery are not Subject to the "July Effect"

Angela Richardson, MD, PhD; Joanna Elizabeth Gernsback, MD; Allan D. Levi, $M D, P h D$

Introduction: The concern that patient outcomes may be worse during the beginning of the academic year when new residents begin their training has been called the "July effect." Several studies have investigated the validity of this concern in patients undergoing neurosurgical procedures without consistent evidence for increased surgical complications.

Methods: We analyzed 5340 prospectively collected consecutive spine cases from a single surgeon over 19 years (January 1997 December 2015). Intradural and post-trauma durotomy cases were excluded a priori.

Results: We identified 4271 cases at risk for an ID, and in 165 of these cases, IDs were reported (3.58\%). The individual primarily involved in the ID was identified, and in our cohort, was $58 \%$ resident, $20 \%$ fellow, and $22 \%$ attending. We examined the rates of ID in our cohort by month and quarter. The rate of incidental durotomy was not significantly different between quarter 3 (July September) and quarter 2 (April June) $3.26 \%$ vs $4.32 \%$ ( $\mathrm{p}=0.2186$ ). Attending surgeons theoretically are not subject to the July effect, however, removing those IDs still failed to demonstrate a difference. Revision surgeries have higher reported rates of ID $(6.5 \%)$, but limiting our analysis to initial surgeries also did not demonstrate evidence of increased ID at the beginning of the academic year. Although not significant, the rate of ID in quarter 3 is consistently lower than in quarter 2 in all these analyses.

Conclusion: Our data suggests that while the July effect makes splashy headlines, it does not apply to incidental durotomies after spine surgery in this prospective, single surgeon consecutive series.

352. Nested Transforaminal Lumbar Interbodies: Increasing the Dimensions of the Interbody Footprint to Match Transpsoas and Anterior Graft Size

\section{Luis Manuel Tumialan, MD; Angelina N. Garvin, BA; Frederick F. Marciano, $M D$}

Introduction: Transpsoas and anterior lumbar approaches offer larger access corridors into the interbody space. The inevitable anatomical circumstance is a larger graft that can span the entire disc space with a greater volume for interbody fusion. Kambin's triangle makes the transpsoas corridor inherently more constrained thereby limiting the size of a graft. The authors recently published a cadaveric anatomical feasibility and biomechanical study demonstrating the capacity to secure sequential interbody spacers in a nested fashion. The goal of the study is to assess the safety, efficacy, radiological and clinical outcomes of the nested transforaminal technique.

Methods: A retrospective analysis from a prospectively collected database of 73 consecutive patients who underwent the nested transforaminal lumbar interbody technique was undertaken. Neurological status before and after surgical intervention, operative times, blood loss, hospital stay, preoperative and postoperative ODI, VAS and SF-36 were evaluated. Radiographic parameters were analyzed for segmental lordosis, foraminal height and arthrodesis.

Results: Among the 73 patients there was no neurological deficits. Operative time, blood loss and hospital stay remained the same when compared to an age and level matched cohort of single interbodies. One patient experienced a graft extrusion, but remained asymptomatic without the need for additional surgery. $96 \%$ achieved a radiographic arthrodesis at 6 months compared to $92 \%$ of single interbodies at 6 months.

Conclusion: The nested transforaminal interbody technique is a safe and effective in increasing the volume of the interbody graft, approaching the volume size of transpsoas and anterior approaches. A nested interbody doubles the volume of the interbody graft, better maintains foraminal height while allowing for more loading of the interbody graft to optimize Wolff's Law and minimize the risk of graft migration or extrusion. The nested interbody technique may be associated with a slightly earlier fusion.

353 Is Three Months an Appropriate Time to Evaluate Clinical Outcomes After Anterior Cervical Discectomy and Fusion?

\section{Marcus D. Mazur, MD; Erica Fay Bisson, MD, MPH}

Introduction: The optimal time to assess patient-reported clinical outcomes after anterior cervical discectomy and fusion (ACDF) has not been established. Although long-term outcomes (i.e., $>=12$ months) are desirable, obtaining follow-up data is time-consuming and expensive, and it may not be necessary for some situations. We evaluated whether 3 months, rather than 12 months, was an appropriate time to assess clinical benefit after ACDF.

Methods: Prospective data for the N2QOD registry were collected from patients undergoing ACDF at our institution from 2013 to 2015. Baseline, 3-month, and 12-month Neck Disability Index (NDI) scores were recorded. A change of -7.5 in NDI score has previously been defined as minimal clinically important difference (MCID). Patients reported their level of satisfaction with the surgical outcome at 3 and 12 months. Satisfied patients reported that surgery met expectations or that they would undergo the same operation for the same results. Unsatisfied patients reported that they would not undergo the same operation for the same results or they were the same or worse as compared with before surgery.

Results: Of 180 patients included, 169 (94\%) were satified at 3 months. Of these, $160(95 \%)$ continued to be satisfied at 12 months. Satisfied patients had a mean change in NDI score at 3 months that was greater than MCID, but unsatisfied patients did not (-9.9 vs. $-1.7, p<0.01)$. The proportion of satisfied patients with a change in NDI score greater than the MCID was similar at 3 and 12 months $(58 \%$ vs. $62 \%$, respectively, $\mathrm{p}=0.50)$, as was the proportion of unsatisfied patients with a change in score less than MCID (73\% vs. $50 \%$, respectively, $\mathrm{p}=0.40$ ). There was good correlation between 3 - and 12-month NDI scores for both satisfied $(\mathrm{r}=0.66, \mathrm{p}<0.01)$ and unsatisfied groups $(\mathrm{r}=0.80, \mathrm{p}<0.01)$.

Conclusion: Three months is an appropriate time for evaluating clinical outcomes after ACDF.

354. 30-Day Outcomes of Thoracolumbar Spinal Fusion Surgery in Extreme Obesity

Jacob R. Joseph, MD; Brandon Smith, MD, MSCR; Jennifer Neva; M. Oakley Strasser; Xilin Liu; Yamaan Saadeh, MD; Paul Park, MD

Introduction: Recent studies have suggested that obese patients have poorer outcomes after thoracolumbar spinal fusions. Here, we examined 30-day outcomes in patients with BMI $>=40$ (defined as extreme obesity by the CDC).

Methods: A retrospective chart review of spinal fusion surgery performed at our institution between 2006 and 2016 was executed. All patients with preoperative BMI $>=40$ were included. Baseline patient characteristics including age, gender, BMI, ASA, CCI, smoking, preoperative narcotic use, and depression were collected. Detailed procedural data was collected, as was 30-day outcomes including complications, readmissions, reoperations, and mortality.

Results: 56 patients with extreme obesity undergoing thoracolumbar fusion were identified. Mean age was 55.7(range 31-74), with 26(46\%) females and 30(54\%) males. Mean BMI was 44.2 (range 40.0 - 54.7). Mean ASA was 2.7 (range 2 3), and mean 
CCI was 1.1 (range 06 ). Seven patients (12.5\%) were active smokers, and $73.2 \%$ of patients used narcotics preoperatively. $51.8 \%$ of patients were on depression medication. Mean number of fused levels was 3.3 (range 215 ). Minimally invasive techniques were used on $35.7 \%$ of patients. Mean EBL was $744+/-924.37 .5 \%$ of patients had at least 1 complication. Mean LOS was 4.4+/-2.1. Mean number of complications was $0.8+/-1.2$. 30-day all cause readmission rate was $5.4 \%, 30$-day reoperation rate was $3.5 \%$. There were no deaths.

Conclusion: Extremely obese patients undergoing thoracolumbar fusion experienced similar 30-day outcomes when compared to the literature on non-obese and obese patients.

355. Trends in the Use of Patient-Reported Outcome Instruments in Neurosurgical Adult Thoracolumbar Deformity and Degenerative Disease Literature

Hanna Algattas, MD; Jonathan A. Cohen, BA; Nitin Agarwal, MD; D. Kojo Hamilton, $M D$

Introduction: Currently shifting national healthcare trends have placed an increased emphasis on patient-centered care and valuebased outcome measures. With this trend, patient-reported outcome instruments (PROIs) are more commonly being used as a surrogate to objectively quantify the value of healthcare offered. We sought to characterize the trends in PROI use over the past decade with regards to thoracolumbar degenerative spine disease and spinal deformity in major neurosurgical journals.

Methods: Articles were screened for PROI use via a PubMed search among five major neurosurgical journals from 2006 to 2016. Articles focusing on adult thoracolumbar deformity and degenerative disease were selected with at least a neurosurgeon included in authorship. Articles meeting inclusion and exclusion criteria were further characterized concerning PROI use, instruments used, and utilization over time.

Results: A total of 29 different PROIs were used among 102 articles identified from 2006 to 2016 using our search strategy. Journal of Neurosurgery: Spine contained the most articles utilizing PROIs with $35.3 \%$ of all articles meeting search criteria. The most frequently used PROIs were Oswestry Disability Index (ODI), Visual Analog Scale (VAS), and the European Quality of Life 5 Dimension Questionnaire (EuroQoL-5D) used in $79.4 \%, 59.8 \%$, and $29.4 \%$ of articles, respectively. Linear regression identified a significant increase in the number of articles employing PROIs from 2006-2016 $(\mathrm{y}=1.85, \mathrm{R} 2=0.77, \mathrm{p}<0.01)$. The total number of PROIs per article was relatively stagnant over time and did not significantly change $(y=0.03, R 2=0.05, p=0.51)$.

Conclusion: The use of PROIs as an outcome tool in the adult thoracolumbar disease literature has increased during the past decade. This trend may be indicative of its use to define patient expectations, with respect to optimum outcomes. There is also, a trend towards its use as a surrogate measure of value-based care.

356. Fusion Rate, Dysphagia and Patient Satisfaction for 4 level Anterior Cervical Decompression and Fusion (ACDF)

Ratnesh Nandan Mehra; Richard F. Cook, DO; Doris Tong, MD; Teck Mun Soo

Introduction: The literature on $\mathrm{ACDF}>3$ levels remains controversial. Combined anterior posterior cervical fusion leads to increased morbidity and loss of cervical mobility. We investigated fusion rate, incidence of dysphagia and patient satisfaction in 4-level $\mathrm{ACDF}$ to demonstrate that multi-level ACDF $>3$ levels is a reasonable option.

Methods: Radiographic and chart review were done for consecutive patients who underwent elective 4-level ACDF from 2006-2011. Fusion rate was evaluated for patients with postoperative cervical radiographs/CT at 6 months or more. We excluded patients with a planned staged anterior-posterior cervical fusion. Fusion is determined by neuroradiologists who evaluated post-operative standing radiographs and $\mathrm{CT}$. The criteria are lucency around the hardware, absence of spinous process movement on flexion/extension films, and bone bridging. Patients completed a dysphagia and satisfaction survey by phone. Descriptive statistics were used.

Results: Ninety-three patients were included in the study. Eightytwo patients had postoperative imaging at 6 months or more. There were a total of 328 interspaces; 316 interspaces were adequate for fusion evaluation. Ten interspaces either had failed fusion or indeterminate fusion status. Fusion rate per interspace is $96.8 \%$. Seventy-seven patients had successful fusion. Fusion rate per patient is $93.9 \%$. Patient satisfaction surveys were completed in 60 out of 93 $(64.5 \%)$ patients. Fifty-three $(88 \%)$ out of 60 patients were satisfied with the results of the surgery. Postoperative dysphagia resolved within 4-30 days with an average of 13 days. All patients surveyed regained normal swallowing function.

Conclusion: Four-level ACDF achieves a very high fusion rate in our institution with a very high satisfaction rate and comparable dysphagia rate. ACDF has proved to be a viable and effective alternative for patients with surgical pathology of $>3$ levels.

\section{Prediction of Clinical Outcomes After Transforaminal Lumbar Interbody Fusions Based on Preoperative Opioid Use}

Alan T. Villavicencio, MD; Lee Nelson, MD; Vinod Kantha, BA; Sharad Rajpal, MD; Sigita Burneikiene, MD

Introduction: Opioid analgesics have become one of the most prescribed drugs in the world, despite the lack of long-term studies evaluating benefits vs. risks for chronic conditions and the fact that long-term use may be associated with worse long-term clinical outcomes. The primary objective of this study was to identify if preoperative opioid use predicted inferior clinical outcomes in patients undergoing transforaminal lumbar interbody fusion.

Methods: A prospective observational study was performed. A total of 93 patients were enrolled undergoing one- to two-level transforaminal interbody fusions and divided into two groups based on preoperative opioid use. The preoperative and clinical outcomes scores at 12-month follow-up were compared.

Results: A total of $60(64.5 \%)$ patients preoperatively used prescribed opioid medications. The patients who preoperatively used opioid medications had significantly higher low back $(p=0.016)$ VAS scores, more disability $(\mathrm{p}=0.013)$, and lower health-related quality of life PCS scores $(p=0.03)$ at the 12 months follow-up. The SF-36 MCS scores remained significantly lower $(p=0.035)$ in the opioid user group, but this was due to the significantly lower baseline scores.

Conclusion: The use of opioid medications to control pain symptoms prior to undergoing lumbar fusion for degenerative lumbar conditions was associated with less favorable clinical outcomes postoperatively. This is the first study that has demonstrated this association in a homogeneous population of patients, which should be studied further to confirm these conclusions. 
358. Sitting Radiographs Use is a Novel Preoperative Planning Tool in Patients Undergoing Surgery for Degenerative Spinal Disease

Muhammad Burhan Janjua; Jared Tishelman; Gregory W. Poorman; Aaron James Buckland, MBBS, FRACS; Themistocles Protopsaltis, $M D$

Introduction: Adult degenerative spinal disease demands a detail surgical planning for an optimal outcome. Surgeons typically plan surgery based upon on the standing radiographs, however, more time is spent sitting than standing in the majority of these patients. Using preoperative global standing, and supine radiographs is universal as body positioning affects sagittal alignment. There is a paucity of information regarding the utilization of sitting radiographs in preoperative planning and assessment; we therefore, present a unique and novel idea of utilizing sitting radiographs as a part of preoperative planning.

Methods: Patients undergoing instrumentation and correction surgery for degenerative spinal disease were retrospectively studied at our institution (2015-2016). Sitting radiographs were used as a part of preoperative planning in all patients, and all sitting and standing radiography were obtained. The following radiographic parameters were analyzed in preoperative sitting and standing imaging: Pelvic Tilt (PT), Sacral Slope (SS), Lumbar Lordosis (LL|L1-S1), Pelvic Incidence (PI), Pelvic Incidence minus Lumbar Lordosis (PI-LL), T1 Pelvic Angle (TPA), Thoracic Kyphosis (TK|T1-T12), and Sagittal Vertical Axis (SVA). Paired samples t-tests were used to compare the means of the dependent variables in both radiographic positions.

Results: We identified 67 patients (61.52y.o. $\pm 12.7 ;$ 59.7\% Female). Patients' sitting radiographs demonstrated significant differences in spinopelvic alignment: $10^{\circ}$ higher PT (Sit: $26.48^{\circ}$ vs. Stand: $16.21^{\circ}, \mathrm{p}<0.001$ ) and $15.5^{\circ}$ higher PI-LL mismatch (Sit: $15.78^{\circ}$ vs. Stand: $\left.0.21^{\circ}, \mathrm{p}<0.001\right)$. Sitting radiographs displayed large changes in global alignment as well, as illustrated by TPA (Sit: $24.7^{\circ}$ vs Stand: $13.40^{\circ}, \mathrm{p}<0.001$ ) and SVA (Sit: $52.94 \mathrm{~mm}$ vs. Stand: $22.03 \mathrm{~mm}$, $\mathrm{p}<0.001)$. Table 1 depicts comparisons between sitting and standing radiographic parameters.

Conclusion: The traditional preoperative planning is performed with the standing radiographs. Key alignment parameters PT, PI-LL mismatch, TPA and SVA change significantly from sitting to standing position (changes in LL drive changes in PT). Sitting radiographs may be an important adjunct of preoperative planning for correction in patients with sagittal malalignment.

\section{Preoperative Hyponatremia and Perioperative Complications in Cervical Spinal Fusion}

Thomas Thallner Bomberger, BA; Joseph E. Tanenbaum, BA; Daniel Lubelski, MD; Edward C. Benzel, MD; Michael P. Steinmetz, MD; Thomas E. Mroz, MD

Introduction: Preoperative hyponatremia has been identified as an independent risk factor for poor outcomes in general and specific surgical cohorts. However, the relationship between preoperative hyponatremia and postoperative outcomes among cervical spinal fusion patients is unknown. The purpose of this study is to investigate whether preoperative hyponatremia is a risk factor for 30-day major morbidity and mortality, increased hospital length of stay, and 30-day readmission and reoperation rates in patients undergoing cervical fusion.

Methods: We identified patients using validated CPT codes for cervical fusion in the American College of Surgeons' NSQIP database, and identified hyponatremic $([\mathrm{Na}]<135 \mathrm{mEq} / \mathrm{L})$ and normonatremic ([Na] $135-145 \mathrm{mEq} / \mathrm{L})$ groups. We performed a univariable analysis to identify potentially predictive covariates in the NSQIP data, and addressed missing data using multiple imputation. A unique multivariate logistic regression model was used to identify significant associations between hyponatremia and several outcome measures, when compared to the normonatremic group.

Results: 23,858 patients met the inclusion criteria. After adjusting for differences in surgical approach and setting, patient demographics, and numerous comorbidities, we found that preoperative hyponatremia was significantly associated with increased odds of major morbidity and mortality (OR 1.23; 95\%CI 1.09-1.39), major morbidity (OR 1.24; 95\%CI 1.10-1.41), mortality (OR 1.36; 95\%CI 1.04-1.78), longer hospital length of stay (OR 1.14; 95\%CI 1.04-1.25), and increased odds of re-operation (OR 1.16; 95\%CI 1.00-1.36).

Conclusion: The present multi-institution study found that preoperative hyponatremia was independently associated with an increased risk of poor perioperative outcomes after controlling for numerous confounding covariates. As the United States transitions to a value and quality-based healthcare model, quantifying the association between modifiable risk factors and adverse perioperative outcomes has become increasingly important. The results of the present study can improve patient selection and preoperative risk counseling for cervical spinal fusion operations, leading to improved value and quality-related healthcare delivery.

360. FEA Model Demonstrates Fully Expanded TLIF Spacers Provide More Lordosis, Less Foraminal Compression, and More Endplate Stress than Static Spacers: The Relationship to Implant Positioning

Noelle F. Klocke, MS; Wenhai Wang, PhD; Karthik Madhavan, MD; Wilson Z. Ray; Anthony J. Russo, MD; Steven Vanni, DO; Lee Onn Chieng, BS; Ammar H. Hawasli, MD, PhD; Christoph Paul Hofstetter, MD, PhD; Brandon Bucklen, PhD

Introduction: While a static transforaminal lumbar interbody fusion (TLIF) spacer's height may be limited to discrete increments, in situ continuous, precise expansion to the desired height is achievable with expandable interbody technology. In theory, this improves surgical goals of decompression and focal lordotic change, yet additional stress may be placed upon the endplate. Using finite element analysis (FEA), the relationships between focal lordosis and posterior decompression, and focal lordosis and endplate stress were investigated.

Methods: Three titanium TLIF spacers were investigated: (1) static $10 \mathrm{~mm} 0^{\circ}$ spacer (S), (2) a $10-14 \mathrm{~mm} 8^{\circ}$ expandable spacer $\left(\mathrm{E}-8^{\circ}\right)$, and $(3)$ a $10-14 \mathrm{~mm} 15^{\circ}$ expandable spacer $\left(\mathrm{E}-15^{\circ}\right)$. Using a previously validated osteoligamentous L4-L5 segment, a left-sided TLIF surgical technique was simulated by placing the spacers at $75 \%$ and $95 \%$ towards the anterior lip following partial facetectomy and discectomy, and then a vertical compression force was applied to the posterior fixation until full closure (spinous processes touched). Expandable implants were evaluated at their maximum height (14mm). Maximum endplate stresses (normalized to subsidence stress of 40MPa, derived from Hou 2009) and foraminal height were obtained. The resulting focal lordosis was measured between the L4 inferior endplate and the L5 superior endplate.

Results: The intact FEA model had a lordotic angle of $3.8^{\circ}$ and average foraminal height of $21.7 \mathrm{~mm}$. While reduction of the posterior pedicle screws inherently reduced the foraminal height, both expandable spacers lessened the magnitude of compression (Figure). Yet, opposite trends were observed between expandable spacers for positioning and foraminal height. All three devices created more endplate stress and lost lordosis (only S gained lordosis) if they were placed more posteriorly.

Conclusion: The fully expanded TLIF spacers studied herein increased lordosis and foraminal height, but also increased endplate stress. Due to the inverse relationships between E- $8^{\circ}$ and E- $15^{\circ}$, spacer lordosis is another variable that affects these measures. 
361. Assessment of Sagittal Balance Post TLIF - Are We Kyphosing the Lumbar Spine?

Karthik Madhavan, MD; Alan Gregory Shamrock; Lee Onn Chieng, BS; Steven Vanni, DO; Michael Y. Wang, MD, FACS

Introduction: Transforaminal lumbar interbody fusion (TLIF) being "work horse" spine procedure bosting minimally invasive access channel. However, this is leading to suboptimal lordotic interbody cage placement. This is apparent in lower lumbar levels as they contribute significantly to lumbar lordosis. We reviewed our series of patients at our institution to evaluate this hypothesis.

Methods: 730 patients who underwent TLIF surgery were retrospectively reviewed. Standing $\mathrm{X}$ rays were assessed for changes in disc height, lumbar lordosis, pelvic incidence, pelvic tilt, and sacral slope. Reoperation rate was assessed as well.

Results: 730 operations were performed at more than 1000 levels. Mean presenting lumbar lordosis (LL) was $47.0 \pm 14.0$. Mean postop LL was $46.5 \pm 13.0$. Among 90 patients with kyphosis (12.3\%), $45 \%$ had restoration of lordosis. Interestingly, among those with normal lordosis, 57\% developed kyphosis postoperatively. High pelvic tilt (PT), sacral slope (SS), and pelvic incidence (PI) were observed in $57 \%, 8.5 \%$, and $24 \%$ respectively. However, only $32.1 \%$ and $60 \%$ of these patients with high PT and SS were successfully corrected with TLIFs while the high PI was remained unchanged as expected. Moreover, $44.5 \%$ of the patients had PI-LL mismatch $=11 \mathrm{o}$ (mean: $220 \pm 9.1$ ) in which $28 \%$ of these remained not corrected (mean: $22.9 \mathrm{o} \pm 8.5$ ). The reoperation rate was $4.1 \%$. Factors of reoperation included uncorrected LL (OR: 44.0; 95\%CI 2.7, 726; $<<0.0001)$ and uncorrected PT (OR: 4.5; 95\% CI 0.6, 36.5; $\mathrm{p}=0.16$ ).

Conclusion: Several factors influence sagittal balance post TLIF like inherent lordosis of the cage, the expandable nature, anterior position of cage and compression of posterior screws. Spino-pelvic parameters are the foundation column and any insufficient correction leads to "inverted pendulum effect" where the upper body gradually kyphoses forward leading deformity.

362. Predictors of Adjacent Segment Disease in Anterior Lumbar Interbody Fusion and Transforaminal Lumbar Interbody Fusion

Matthew R. Peck, BS; Heath P. Gould, BS; Jeffrey A. O'Donnell, BS; Joseph E. Tanenbaum, BA; Thomas E. Mroz, MD; Michael P. Steinmetz, $M D$

Introduction: Adjacent Segment Degeneration (ASD) is a common morbidity associated with lumbar spine surgery used to treat symptomatic spinal stenosis or spondylolisthesis. Two approaches to lumbar fusion are Anterior Lumbar Interbody Fusion (ALIF) and Transforaminal Lumbar Interbody Fusion (TLIF). Studies have shown similar rates of fusion in the procedures. However, there is no consensus on the relative incidences of ASD in ALIF as opposed to in TLIF. Finding predictors of ASD in ALIF and TLIF would improve surgical decision making and risk counseling.

Methods: The present study is a retrospective study of patients undergoing single-level ALIF or TLIF at the Cleveland Clinic between 2008 and 2012. Only adults undergoing their first lumbar spine surgery were included. Data were collected on patient gender, race, age, BMI, tobacco use, alcohol use, drug use, procedure type, and presence of ASD according to post-operative imaging. Three independent reviewers used Distortion-compensated Roentgen analysis (DCRA) to calculate anterior disc height and posterioranterior displacement before and after the procedure for each patient. ANOVA logistic regression was performed to identify patient characteristics associated with ASD.

Results: 115 patients met our inclusion and exclusion criteria. The average age of our cohort was $56 \pm 12.6$ with 60 men $(52.1 \%)$ and 55 women (47.9\%). 15 subjects (13.0\%) used tobacco, and 62 (53.9\%) were obese $(\mathrm{BMI}>30)$. Surgical indications included spondylolisthesis, spinal stenosis, spondylosis, disk herniation, degenerative disk disease, and discitis. 48 (41.7\%) patients underwent ALIF; 67 (58.3\%) underwent TLIF. ANOVA regression analysis showed that obesity $(\mathrm{p}=0.040)$, spondylosis $(\mathrm{p}=0.029 ; \mathrm{p}=0.024)$, tobacco use $(\mathrm{p}=0.010$; $\mathrm{p}=0.015)$, and TLIF $(\mathrm{p}=0.027)$ were all associated with increased odds of developing ASD associated DCRA value changes.

Conclusion: The present study found TLIF, obesity, spondylosis, and tobacco use were predictors of ASD following lumbar fusion. These results can improve decision-making and preoperative risk counseling.

363. Biomechanical Characterization of an Anterior Cervical Plate Capable of Extreme Oblique Screw Angulation: Does Angulation Affect Construct Rigidity?

Chris M. Ferry, BS, MS; Anup Gandhi, PhD; Sam Farmer; Tom Glorioso, MS; Jason Inzana; John E. Wanebo, MD; Ripul Rajen Panchal, DO

Introduction: Adjacent level ossification following plated anterior cervical discectomy and fusion (ACDF) remains of concern when utilizing the technique. Next-generation plate designs allow for greater oblique screw angulation to help diminish plate profile while still averting the index endplates. However, little consideration has been given to the implications of increased/extreme oblique angulation on construct rigidity. The objective of this study was to biomechanically assess an ACDF plate (Fig.1) capable of 30deg cephalad and 30deg caudal screw angulation to determine whether a correlation may exist between angulation and rigidity.

Methods: Nineteen cadaveric cervical spine specimens (C3T1) were tested. Each spine was first tested in an intact state. An anterior discectomy (C5/C6) was then performed, followed by instrumentation with the novel plate and PEEK cage. Plate size selection and screw angulation was at the discretion of the surgeon. Lateral and A/P images were taken after. Screw angle measurements: 1) angle between cephalad and caudal screws (total sweep) and 2) sum of cephalad and caudal screw angulation relative to affected vertebral endplates. A Pearson correlation for each metric was performed across all principle motion directions. A $2 \mathrm{Nm}$ moment was applied in flexion/extension (FE), lateral bending (LB), and axial rotation (AR). Range-of-motion (ROM) was tracked and reported relative to intact conditions.

Results: Screw sweep ranged from 6.4deg to $60.0 \mathrm{deg}$ (max), with a median of $38.5 \mathrm{deg}$ and a mean of $35.6 \mathrm{deg}$. Summed cephalad/ caudal angulation relative to index endplates ranged from 17.9deg to $73.2 \mathrm{deg}$, with a median of $45.3 \mathrm{deg}$ and a mean of 44.1deg. Analyses indicated no significant correlations between either angulation metric and ROM reduction (screw sweep Pearson correlations: $\mathrm{FE}=-0.12$ (Fig.2); $\mathrm{LB}=0.03 ; \mathrm{AR}=0.004$ ).

Conclusion: The stability of plated ACDF may not be compromised when using extreme oblique screw angulation. The ability to leverage greater angulation to support a smaller stature plate is clinically advantageous.

364. Robot-guided Pedicle Screw Fixation for Lumbar Spondylolisthesis - Long-term Clinical Outcomes and Revisions

Marc L. Schröder, MD, PhD; Victor E. Staartjes

Introduction: The accuracy of robot-guided pedicle screws has been proven to be high, but little is known on revision rates. No reports include long-term patient-reported clinical outcomes. A clinical benefit for the patient is unknown. This is the first report on long-term outcomes of robot-guided pedicle screw fixation for single 
level spondylolisthesis. Literature data were used to compare surgical revision rates and patient reported outcomes.

Methods: We retrospectively analyzed prospectively collected data from patients undergoing Minimally Invasive Transforaminal/ Posterior Lumbar Interbody Fusion (MI-T/PLIF). Patients were followed up at 6 weeks, 12 months, 24 months and by mailed questionnaires in March 2016 as a final follow up. Visual Analog Scale (VAS) for back and leg pain severity, Oswestry Disability Index (ODI), peri- and postoperative revision rates and socio-demographic factors were analyzed.

Results: 72 patients fit our inclusion criteria, and had a mean follow up of $32 \pm 17$ months. Mean improvements in VAS back pain $(49.3 \%)$, leg pain $(63.4 \%)$ and ODI $(61.2 \%)$ were consistent with previous reports. Length of stay was 2.4 days and operating time was 161 minutes. $89.1 \%$ of patients indicated they would choose this treatment again. Ability to work increased from $38.9 \%$ to $78.2 \%$ (p $<$.001). No instrumentation-related complications occurred, and no revision surgery was needed. Intraoperative screw repositioning was significantly lower compared to navigated and free hand techniques $(\mathrm{p}<.001)$. PLIF, high BMI, smoking status, and preoperative ability to work were identified as predictors of reduction in back pain.

Conclusion: Minimally invasive robot-guided pedicle screw fixation is a safe and effective means to treat spondylolisthesis. It lowers intraoperative screw revisions when compared to published evidence on both free hand and navigated procedures. Moreover, it significantly reduces the revision rate for pedicle screw malposition compared to freehand procedures. Although robotic surgery will never fully replace the surgeon, software and hardware have now advanced to the point where trajectories can be defined even more precisely than by an experienced surgeon's hand.

365. Preemptive Analgesia for Postoperative Pain Relief in Thoraco-lumbo-sacral Spine Operations: A Double-blinded, Placebo Controlled Randomized Controlled Trial

Linda S. Aglio, MD; Muhammad M. Abd-El-Barr, MD, PhD; Vwaire Orhurhu, MD, MPH; Grace Kim, BS; Jie Zhou; Laverne Gugino, MD, PhD; Lisa Crossley, MD, PhD; James Gosnell, RN; John Chi, MD, MPH; Michael W. Groff, MD, FACS

Introduction: Preemptive administration of analgesic medication, before the onset of the actual painful stimulus, is more effective than that after the onset of the painful stimulus. Its role in postoperative pain relief after thoraco-lumbo-sacral spine surgery has not been fully investigated

Methods: With IRB approval, we performed a double-blinded randomized controlled trial to analyze the efficacy of preemptive analgesia. Ninety-nine patients who underwent spine surgery in the thoraco-lumbo-sacral spine through the posterior approach, with or without instrumentation, were randomized into three groups. Patients in the control group $(n=32)$ received a single epidural injection of $10 \mathrm{~mL}$ of normal saline. Patients in study group $\mathrm{A}(\mathrm{n}=33)$ received a single epidural injection of $10 \mathrm{~mL}$ solution containing $0.5 \mathrm{mg}$ of hydromorphone alone. Patients in study group $B(n=34)$ received a single epidural injection of $10 \mathrm{~mL}$ solution containing $0.0625 \%$ bupivicaine with $0.5 \mathrm{mg}$ hydromorphone. Primary outcome was presence of opioid sparing and rescue time defined as time interval from when patient was extubated to the time pain medication was first demanded during the postoperative period.

Results: No significant difference was seen across the demographics and surgical complexity for placebo, group A, and group B. Compared to the control group, opioid-sparing was significantly higher in group A $(57.6 \%$ vs. $15.6, \mathrm{p}=0.0007)$ and group $\mathrm{B}(52.9 \%$ vs. $15.6 \%$, $\mathrm{p}=0.0045)$ in the first demand of intravenous hydromorphone as supplemental analgesic medication. There were significant difference in rescue time across all three groups. Across all three groups, there were no significant difference in amount of IV dilaudid used at the PACU, hospital length of stay, VAS score on rescue, and total oxycodone amounts on postoperative days 0,1 , and 2 .

Conclusion: Our trial demonstrated significantly better pain relief in both study groups with preemptive analgesic epidural. Preemptive analgesia with a single shot epidural injection of bupivicaine with hydromorphone or hydromorphone alone is a safe, simple and effective method for postoperative pain relief in patients undergoing thoraco-lumbo-sacral operations.

\section{Impact of Age on Change in Self-Image 5-Years After Complex Spinal Fusion (e"5 Levels)}

Owoicho Adogwa, MD, MPH; Aladine A. Elsamadicy, BE; Amanda Sergesketter; Keith Bridwell, MD; Isaac O. Karikari, MD

Introduction: With an aging population, the impact of age on long-term change in Self-Image is unknown. The aim of this study is to determine the effects of age on ? Self-Image 5-years after undergoing an elective complex spinal fusion (=5-Levels).

Methods: This was a retrospective analysis of prospectively collected data of 55 adult patients $(=18$ years old) undergoing $=5$-levels of spinal fusion to the sacrum with iliac fixation from January 2002 to December 2008. Patients were grouped by age: Young ( $<60$ years old) and older (=60 years old). Patient demographics, comorbidities, preoperative variables and post-operative complication rates were collected. All patients had prospectively collected outcome measures and a minimum of 5-year follow-up. Patient reported outcomes instrument SRS-22r (Function, Self-Image, Mental Health, and Pain) was completed before surgery then at follow-up ( $=5$ year). The primary outcome investigated in this study was the change in SelfImage after surgery.

Results: Baseline characteristics and preoperative variables were similar in both cohorts. There were no significant differences in intraoperative variables, including the mean $\pm \mathrm{SD}$ number of fusion levels between the cohorts (Young: $11.2 \pm 4.3$ vs Older: $12.1 \pm 4.0, \mathrm{p}=0.42$ ). Complication rates were similar between the cohorts, with no significant differences in the types of complications (Young: $29.63 \%$ vs Older: $25.0 \%, \mathrm{p}=0.77$ ). There were no significant differences in preoperative and follow-up patient reported outcomes between the cohorts. The mean $\pm \mathrm{SD}$ preoperative and follow-up self-image scores were (Young: $2.35 \pm 0.58$ vs Older: $2.68 \pm 0.64$ ) and (Young: $3.82 \pm 0.63$ vs Older: $3.51 \pm 0.94$ ), respectively. There were no significant differences in the change of function, mental health, or pain between the cohorts. However, the younger cohort experienced a significantly greater overall change in self-image than the Older cohort (Young: $1.49 \pm 0.87$ vs Older: $0.70 \pm 1.14, p=0.01$ ).

Conclusion: Our study suggests that age significantly affects the perception of self-image after deformity correction surgery; with younger patients reporting a greater change from baseline in selfimage after surgery.

367. Comparisons Between Extraforaminal Approach and Contralateral Approach to Lumbar Foraminal Disc Herniations

\section{Joon Young Ahn; Se Young Pyo; Ho Soo Kim; Yong Tae Jung}

Introduction: Surgical treatments of lumbar foramoinal disc herniations have been varied. Fusion surgery have been a traditional concept for the treatment of these pathologies, or Non-fusion surgery as extraforaminal approach also have been an acceptable concept. Recently facet joint preserving contralateral approach was introduced to the lumbar spinal stenosis with foraminal stenosis. Tubular retractor guided contralateral approach is a kind of minimally invasive techniques for preserving spinal functional units. We tried this procedure on that conditions and simultaneously removal 
of contralateral foraminal herniated discs. The aim of this study was to compare the results between extraforaminal approachs and contralateral approaches, and to give consideration of approach indications.

Methods: From Sep. 2010 to Dec. 2015 extraforalminal approaches of lumbar foraminal disc herniations were underwent 15 patients with unilaterally dominant leg radiculopathy, who were 10 female and 5 male, mean age was 69 years. From Feb. 2012 to Feb. 2016 procedure of bilateral decompression through unilateral approach were done 48 patients with 86 levels. Among these contralalteral discectomies were performed to 10 patients with 13 levels. Patients data were 5 Male and 5 female, mean age was 64 years. Outcome measurements were used Visual analogue scale pre- and post-operatively on pathology side buttock and leg pain, and functional outcomes were evaluated by using the MacNab criteria.

Results: For the extraforaminal approach patients, postoperatively there was significant improvement in VAS back pain and VAS leg pain $(\mathrm{P}<0.001)$. At follow-up of $6.8 \pm 1.7$ months, there was also significant improvement in VAS back pain and VAS leg pain $(\mathrm{P}<$ 0.001). The functional outcome was mostly excellent and good. One patient required revision with same procedure $(6 \%)$. For contralateral approach patients, postoperatively there was slightly improvement in VAS back pain, but significant improvement VAS leg pain $(\mathrm{P}<0.001)$. At follow-up of $6.5 \pm 1.3$ months, there was slightly improvement in VAS back pain, but significant improvement in VAS leg pain $(\mathrm{P}<$ 0.001). The functional outcome was excellent and good in 9 patients, but 1 patient was fair. No one had revision surgery. One was recurred leg pain after surgery 1month because progression of foraminal stenosis.

Conclusion: Contralateral approach can lead central decompression with widening of opposite side foraminal stenosis at the same time. Then in the condition of lumbar spinal stenosis combined with foraminal disc herniation, this procedure should be effective to solve that problems by contralateral discectomy simultaneously. More cases and long term follow up should be needed for over limitation such as extraforaminal disc hernation, recurrence of disc herniation and foraminal restenosis.

\section{Comparative Analysis of Fusionless Surgery Versus Fusion Extension as the Treatment of L5-S1 Adjacent Segment Disease After a Lumbar Fusion Surgery Which Stops at L5}

Un Young Choi; Dal-Sung Ryu; Kyung-Hyun Kim; Jeong-Yoon Park; Sung-Uk Kuh; Dong-Kyu chin; Deun-Su Kim; Yong-Eun Cho

Introduction: Adjacent segment disease is one of the long-term complications after a lumbar fusion surgery. Although an interbody fusion with additional pedicle screw fixation is a reliable surgical technique to terminate the problem, it requires consumption of time and effort. Segment surgery such as only decompression or interbody fusion without rod extension, is much simple to perform but sometimes it leads to more serious problem. This study was conducted to compare the result of surgical method to treat L5 - S1 adjacent segment disease.

Methods: We retrospectively reviewed the medical record of 22 patients who underwent the segmental surgery due to adjacent segment degeneration at L5 / S1 segment after a lumbar fusion surgery which stops at L5. They were divided into two groups depending on the segment of initial fusion (single vs multi-level). Clinical outcomes included pain scales at follow up and whether the patient had invasive procedure or not.

Results: The population consisted of 11 men and 11 women. Mean age at the time of second surgery was $61.7 \pm 6.8$. The interval between first fusion surgery and second surgery due to adjacent segment disease ranged from 2 to 644 months. Seventeen patients ( $77.3 \%$ ) complained significant radiculopathy ( VAS $>3$ ) on follow up. After all, 9 patients ( $40.9 \%$ ) underwent revision surgery at L5 / S1 and
4 patients ( $18.2 \%$ ) received epidural steroid injection to control radiculopathy. Only 5 patients ( $22.7 \%$ ) were pain-free on last follow up. Multi-level fusion group showed the incidence of radiculopathy as $11 / 14$ patients ( $78.6 \%$ ) whereas single level fusion group did as $2 / 8$ patients ( $20 \%)$.

Conclusion: A segmental surgery to treat L5-S1 adjacent segment disease showed high re-operation rate and incidence of radiculopathy on follow up. Spine surgeon should be aware of higher risk of problems, especially in initial multi-level lumbar fusion surgery.

\section{Biomechanical Analysis of S2 Alar Iliac (S2AI) Screw Fixation}

Sung Hyun Noh; Man-Kyu Choi; Dong Ah Shin; Kyung-Hyun Kim; Jeong-Yoon Park; Sung-Uk Kuh; Dong-Kyu Chin; Keun-Su Kim; Yong-Eun Cho

Introduction: Recently S2 alar iliac (S2AI) could be considered an alternative method of lumbosacral fixation. Some authors reported that it could address disadvantages of traditional iliac screw fixation such as separate fascial incisions with extensive dissection, the use of offset connectors, and painful prominent screw heads. However there were also mechanical failure especially screw fracture unexpectedly happened during follow-up period, thus its nature of techniques through sacroiliac joint, we hypothesized that the mobility at the sacroiliac joint could make more mechanical stress to the screw-head interface. This study was designed to determine the mechanical stress of S2AI screw according to sacroiliac motion using finite element biomechanical analysis.

Methods: Finite element models of Sacroiliac fixation were created in this study. Modelling was based on fixation with a single S1 screw and S2AI which were connected by a beam element at each side for making effect of rod assembly. Bilateral acetabulum was fixed by Encastre. $400 \mathrm{~N}$ concentrated forces were loaded on the S1 screw heads. 10 steps were done for single simulation. Von Mises stress was measured at the S1 and S2AI screws and vertebral bodies during flexion, extension, and torsion.

Results: Finite element models of this analysis were validated with previous proven model. All displacement during superior, inferior, anterior posterior, flexion, extension, and torsion were within $10 \%$ compared with previous confirmed model. According to our test, maximal Von Mises stress of S2AI was $259.7 \mathrm{Mpa}$ and maximal measured area was the waist of the screw which could be the portion between sacrum and ilium. Maximal Von Mises stress of S1 screw was $259.7 \mathrm{Mpa}$ and maximal measured area was the neck of the screw.

Conclusion: Given our results of 3D-Finite Element analysis, S2AI screw fixation for sacropelvic fixation has biomechanical weakness at the waist of the screw which is located at sacroiliac joint. Thus, screw fracture could be prevented if we make this area enhanced by thickened diameters or biomechanical superior materials.

\section{Contralateral Approach for the Treatment of Lumbar Spinal Stenosis in Combination with Foraminal Disc Herniations.}

\section{Se Young Pyo; Sang Hyeong Jun; Ho Soo Kim; Young Tae Jung}

Introduction: Surgical treatments of lumbar spinal stenosis combined with foraminal disc herniations have been varied. Fusion surgery have been usual concept for the treatment of these pathologies, or Non-fusion surgery as two incision approach, central decompression through midline approach and simultaneously foraminal discectomy through extraforaminal approach also have been accepted. But for preserving facet joints and for avoiding fusion surgery, contralateral approach is considerable. Tubular retractor guided contralateral 
approach is a kind of minimally invasive technique for preserving spinal functional units. We tried this procedure on that conditions and simultaneously removal of contralateral foraminal herniated discs. The aim of this retrospective study was to evaluate the efficacy and benefits of this approach for treatment of lumbar spinal stenosis in combination with foraminal disc herniations.

Methods: From Feb. 2012 to Feb. 2016 procedure of bilateral decompression through unilateral approach were done 48 patients with 86 levels. Among these contralalteral discectomies were done 10 patients with 13 levels. Patients data were 5 Male and 5 female, mean age was 64years. Operating levels were L2/3 2level, L3/4 2level, L4/5 6levels, and L5/S1 3levels. Exclusion criteria is non-degenrative lumbar spinal stenosis, extraforaminal stenosis, instability and deformity. Outcome measurements were used Visual analogue scale pre- and post-operatively on pathology side buttock and leg pain, and functional outcomes were estimated by using MacNab criteria.

Results: Postoperatively there was slightly improvement in VAS back pain, but significant improvement VAS leg pain $(\mathrm{P}<0.001)$. At follow-up of $6.5 \pm 1.3$ months, there was slightly improvement in VAS back pain, but significant improvement in VAS leg pain $(\mathrm{P}<0.001)$. The functional outcome was excellent and good in 9 patients, but 1 patient was fair. No one had revision surgery. One was recurred leg pain after surgery 1month because progression of foraminal stenosis.

Conclusion: Contralateral approach can lead central decompression with contralateral discectomy at the same time. Then in the condition of lumbar spinal stenosis combined with foraminal disc herniation, this procedure suggested effective to solve multiple problems such as lumbar spinal canal stenosis combined with foraminal disc herniation. More cases and long term follow up should be needed for over limitation such as extraforaminal disc hernation, recurrence of disc herniation and foraminal stenosis.

371. Comparison of Radiologic Outcomes of Three Different Methods in Single-level Anterior Cervical Discectomy and Fusion; Stand-alone Cage Versus PEEK-titanium Combined Anchored Cage Versus Anterior Cervical Plate Fixation

\section{Jong Uk Hwang; Geun Sung Song; Dong Wuk Son}

Introduction: Anterior cervical discectomy and fusion (ACDF) is a choice of surgical procedure for cervical degenerative diseases associated with radiculopathy or myelopathy. However, the patients undergoing ACDF still have problems. The purpose of the present study is to evaluate the radiologic results of three different methods in single-level anterior cervical discectomy and fusion.

Methods: We conducted a retrospective collection of radiological data from January 2011 to December 2014. A total of 67 patients were included in this study. The patients were divided into 3 groups by operation procedure: (1) using stand-alone cage (Group Cage, $\mathrm{N}=20$ ); (2) PEEK-titanium combined anchored cage (Group AC, N=21); and (3) anterior cervical cage-plate (Group $\mathrm{CP}, \mathrm{N}=26$ ). Global cervical lordosis (C2-C7 Cobb's angle), fused segment height, fusion rate, and cervical ROM were measured and analyzed at serial preoperative, postoperative, 6-month, and final 1-year follow-up.

Results: Successful bone fusion was achieved in all patients at the final follow-up examination; however, the loss of disc height over $3 \mathrm{~mm}$ at the surgical level was observed in 6 patients in Group Cage. Groups AC and CP yielded significantly better outcomes than Group Cage in fused segment height and cervical ROM ( $p=0.01$ and $\mathrm{p}=0.02$, respectively). Furthermore, Group AC had similar radiologic outcomes to those of Group CP.

Conclusion: The PEEK-titanium combined anchored cage may be a good alternative procedure in terms of reducing complications induced by plate after ACDF.
372. The Effect of Uncinate Process Resection on Subsidence Following Anterior Cervical Discectomy and Fusion

Dong Wuk Son; Geun Sung Song; Sang Weon Lee; Jun Seok Lee; Jong Wuk Hwang; Su Hun Lee; Sun Ki Sung

Introduction: The effects of uncinate process resection (UPR) on cervical spine instability vary between in vivo and in vitro studies. To estimate the in vivo effects of UPR in the context of anterior cervical discectomy and fusion (ACDF), we evaluated the clinical and radiological outcomes associated with subsidence (a proxy for micromotion) after ACDF with bilateral UPR.

Methods: We retrospectively collected clinical and radiological data from January 2011 to June 2015. A total of 29 patients (41 segments) were included in this study. All procedures included bilateral UPR and anterior plate fixation. Reformatted coronal computer tomography images were used to evaluate the resection rate of UPs. To reduce level-related bias, we converted the area of UPR to a proportion: the area of UPR to the pre-operative area of the vertebral body or the pre-operative proportional area of the UP (pUPR).

Results: The sum of bilateral pUPR $(\mathrm{P}=0.006)$ correlated with subsidence. Regression analysis revealed that subsidence could be estimated with the following formula: subsidence $=0.896+3.980 *$ sum of pUPR. The subsidence rate was $34 \%$.

Conclusion: If the sum of pUPR is $>52.9 \%$, the possibility of subsidence increases. This threshold could be useful in future studies and clinical practice, and should be further confirmed by large-scale, prospective studies.

373. Randomized Controlled Study of Percutaneous Epidural Neuroplasty Using Racz Catheter and Epidural Steroid Injection in Cervical Disc Disease

\section{Chang Hyun Oh; Gyu Yeul Ji; Dong Ah Shin}

Introduction: The efficacy of lumbar percutaneous epidural neuroplasty (PEN) as a minimally invasive technique has been relatively well investigated, but the clinical effectiveness of cervical PEN (C-PEN) has yet to be established. The purpose of this study was to compare clinical outcomes between C-PEN and cervical epidural steroid injection (C-ESI).

Methods: Eighty patients with neck pain from single level cervical disease with and without radiculopathy were included in this study. Patients were randomly assigned into 2 groups: C-PEN or C-ESI. Clinical outcomes were assessed according to Neck Disability Index (NDI) score and Visual Analog Scale (VAS) score for arm pain until 12 months after treatment.

Results: All C-PEN and C-ESI groups showed better NDI recovery and greater reduction in VAS score at postoperative 6 months $(\mathrm{P}<0.001)$. The $\mathrm{C}-\mathrm{PEN}$ group demonstrated better NDI score at postoperative 6 months than the $C$-ESI group $(P=0.014)$, while there were no differences at 2, 4, and 12 months. Additionally, the C-PEN group showed lower VAS scores at all follow-up intervals compared to the C-ESI group $(\mathrm{P}<0.050)$. Symptom relief was sustained for a significantly longer duration in the C-PEN group than in the C-ESI group (23.4 vs. 20.5 weeks, $\mathrm{P}<0.001)$.

Conclusion: C-PEN was superior to C-ESI in terms of better NDI recovery (at 6 months) and greater reduction in VAS score (until 12 months) in treating single level cervical disc herniation. Better outcomes with C-PEN may have been achieved via a more localized selective block in the epidural space closer to the dorsal root ganglion and ventral aspect of the nerve root. 
374. Clinical Course of Cervical Percutaneous Epidural Neuroplasty in Single Level Cervical Disc Disease with 12-month Follow-up

\section{Chang Hyun Oh; Gyu Yeul Ji; Dong Ah Shin}

Introduction: Cervical disc disease is a common and occasionally disabling condition, occurring as a natural consequence of aging in the vast majority of the adult population. Percutaneous epidural neuroplasty (PEN) has been used in chronic neck pain to deliver highly concentrated drugs and to prevent scarring in cases refractory to conventional epidural blocks. However, the clinical course after PEN in cervical disc disease is not well documented. The purpose of this study was to evaluate the effectiveness of cervical PEN in single level cervical disc disease.

Methods: Consecutive series of 100 patients with who underwent cervical PEN for single level disc disease (bulging or protrusion) were included in this study. Preoperatively, all patients underwent magnetic resonance imaging (MRI), and visual analog scale (VAS) score and Odom's criteria were measured preoperatively and at postoperative follow-up visits (1, 3, 6 and 12 months).

Results: Additional block therapy was performed in 58 patients (58.0\%). Subsequent surgery was undertaken in 10 patients $(10.0 \%$, excluded from data of clinical followup). Mean neck pain and VAS arm pain scores for all follow-up patients decreased from 6.82 and 4.74 preoperatively to 2.18 and 1.87 at 12 months after PEN ( $p<$ 0.001 ). More than $80 \%$ and $40 \%$ of all patients with and without additional block therapy after cervical PEN showed good and excellent outcomes according to Odom's criteria during 12 months of follow-up. During this follow-up period, there was no severe complication related the procedure was not observed.

Conclusion: Cervical PEN was shown to be a safe and effective treatment for neck and arm pain due in single level disc disease during 12 months of follow-up.
375. Triple Injection Therapy Including Neuropathy, Musculopathy, and Enthesopathy Improved Non-specific Chronic Lower Back Pain After Post Surgery Syndrome

\section{Chang Hyun Oh; Seung Hwan Yoon}

Introduction: The pathology of post-surgery pain syndrome is obscure. Various approach were applied to manage the post-surgery pain syndrome, but its clinical results were limited. The authors considered that multiple pain origin in post-surgery pain syndrome, and applied multiple therapies to these patients.

Methods: In this retrograde observational study, 39 patients after spinal fusion operation were included. The pain origin was considered as central lumbar nerve, muscle and enthesis of back muscles. Scheduled injectional therapy such as caudal epidural steroid injection, trigger point injection and prolotherapy were applied. Total schedule were 8 times injection with each one week interval.

Results: Clinical score using reduction visual anlog scale were significantly decreased from 10 to 3.7 after complete the injection schedule. Functional SF-12 score was also significantly improved until the follow-up period ( 3 months).

Conclusion: Triple injection therapy including neuropathy, musculopathy, and enthesopathy could be useful treatment in nonspecific chronic lower back pain after post surgery syndrome.

Disclaimer: The Journal of Neurosurgery Publishing Group (JNSPG) acknowledges that the preceding abstracts are published verbatim as submitted and did not go through either the JNSPG's peer-review or editing process. 